\title{
Copper-Catalyzed Enantioselective Reductive Cross-Coupling of Aldehydes and Imines
}

Atsuhisa Mitsui, Kazunori Nagao, and Hirohisa Ohmiya*

Division of Pharmaceutical Sciences, Graduate School of Medical Sciences, Kanazawa University, Kakuma-machi, Kanazawa 920-1192, Japan

*Hirohisa Ohmiya: ohmiya@p.kanazawa-u.ac.jp

\section{Table of Contents}

- Instrumentation and Chemicals

$\mathrm{S} 2$

- Characterization Data for Imines and Ketimines

$\mathrm{S} 3-\mathrm{S} 6$

- Procedure for Copper-Catalyzed Enantioselective Reductive

Cross-Coupling between Aldehydes And Imines or Ketimines

S6

- Characterization Data for Chiral $\beta$-Aminoalcohols

$\mathrm{S} 7-\mathrm{S} 32$

- Procedure for Deprotection and Determination of Absolute Configuration

S33

- Scale up Reaction

S34

- References

S35

- NMR Spectra

S36-S111 


\section{- Instrumentation and Chemicals}

NMR spectra were recorded on a JNM-ECS400, operating at $400 \mathrm{MHz}$ for ${ }^{1} \mathrm{H}$ NMR and 100.5 $\mathrm{MHz}$ for ${ }^{13} \mathrm{C} \mathrm{NMR}$, and JNM-ECA600, operating at $600 \mathrm{MHz}$ for ${ }^{1} \mathrm{H} \mathrm{NMR}$ and $150.9 \mathrm{MHz}$ for ${ }^{13} \mathrm{C}$ NMR. Chemical shift values for ${ }^{1} \mathrm{H}$ and ${ }^{13} \mathrm{C}$ are referenced to $\mathrm{Me}_{4} \mathrm{Si}$ and the residual solvent resonances, respectively. Chemical shifts are reported in $\delta$ ppm. Mass spectra were obtained with JMS-T100TD (DART). TLC analyses were performed on commercial glass plates bearing 0.25-mm layer of Merck Silica gel 60F 254 . Silica gel (Kanto Chemical Co., Silica gel 60 N, spherical, neutral) was used for column chromatography. IR spectra were measured with a Thermo Scientific iD7 ATR Accessory for the Thermo Scientific Nicolet iS5 FT-IR Spectrometer. HPLC analyses were conducted on a HITACHI Chromaster with a HITACHI 5430 diode array detector. Melting points were measured on a Yanaco MP-500D apparatus. Optical rotations were measured on a JASCO P-2200.

All reactions were carried out under nitrogen atmosphere. Materials were obtained from commercial suppliers or prepared according to standard procedures unless otherwise noted. $\mathrm{CuCl}$ was purchased from Kanto Chemical Co., stored under nitrogen, and used as received. $\mathrm{NaOt} \mathrm{Bu}$ was purchased from Tokyo Chemical Industry Co., stored under nitrogen, and used as received. $\mathrm{NaOSiMe}_{3}$ was purchased from Aldrich Chemical Co., stored under nitrogen, and used as received. Toluene and THF were purchased from Fujifilm Wako Pure Chemical Co., and purified by passage through activated alumina under positive argon pressure as described by Grubbs et al. $\mathrm{PhMe}_{2} \mathrm{SiB}$ (pin) was purchased from Fujifilm Wako Pure Chemical Co., stored under nitrogen, and used as received. Cyclooctane was purchased from Aldrich Chemical Co., stored under nitrogen, and used as received. DME was was purchased from Tokyo Chemical Industry Co., stored under nitrogen, and used as received. Aldehydes 1a-1k were purchased from Tokyo Chemical Industry Co., stored under nitrogen, and used as received. Imines $\mathbf{2} \mathbf{a}-\mathbf{2 l}$ were prepared by the reported procedure. ${ }^{1}$ Ketimine $\mathbf{2} \mathbf{m}$ was prepared by the the reported procedure. ${ }^{2}(S, S)-\mathbf{L} \mathbf{1} \cdot \mathrm{HBF}_{4}$ was prepared by the reported procedures. ${ }^{3}$ 


\section{- Characterization Data for Imines and Ketimines"}

\section{(E)-N-[2-(2-Ethoxyethoxy)phenyl]-1-phenylmethanimine (2e)}

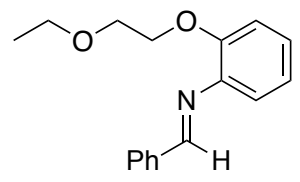

$2 e$

The imine $2 \mathrm{e}$ was purified by distillation (10 mmol scale, $1.54 \mathrm{~g}, 57 \%$ isolated yield.). The compound is contaminated with inseparable impurities. Yellow oil. IR (neat) 759, 1116, 1247, 1487, 1629, 2869, 2925, 2973, 3028, 3061, $3442 \mathrm{~cm}^{-1}$. ${ }^{1} \mathbf{H}$ NMR $\left(600 \mathrm{MHz}, \mathrm{CDCl}_{3}\right) \delta 1.16(\mathrm{t}, J=7.2 \mathrm{~Hz}$, $3 \mathrm{H}), 3.58(\mathrm{q}, J=7.2 \mathrm{~Hz}, 2 \mathrm{H}), 3.79(\mathrm{t}, J=5.4 \mathrm{~Hz}, 2 \mathrm{H}), 4.19(\mathrm{t}, J=5.4 \mathrm{~Hz}, 2 \mathrm{H}), 6.98-7.05(\mathrm{~m}, 3 \mathrm{H})$, $7.15(\mathrm{~m}, 1 \mathrm{H}), 7.45-7.48(\mathrm{~m}, 3 \mathrm{H}), 7.90-7.92(\mathrm{~m}, 2 \mathrm{H}), 8.50(\mathrm{~s}, 1 \mathrm{H}) .{ }^{13} \mathbf{C} \mathbf{N M R}\left(150.9 \mathrm{MHz}, \mathrm{CDCl}_{3}\right) \delta$ 15.1, 66.8, 68.8, 68.9, 114.3, 121.1, 121.6, 126.2, 128.5, 128.6, 131.0, 136.4, 142.2, 151.1, 161.4. HRMS-DART $(\mathrm{m} / \mathrm{z})$ : $[\mathrm{M}+\mathrm{H}]^{+}$calcd for $\mathrm{C}_{17} \mathrm{H}_{20} \mathrm{~N}_{1} \mathrm{O}_{2}, 270.1489$; found, 270.1484 .

\section{(E)-N-\{2-[2-(2-Methoxyethoxy)ethoxy]phenyl\}-1-phenylmethanimine (2f)}

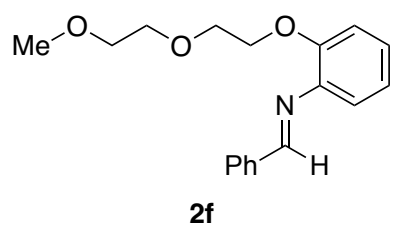

The imine $2 \mathbf{f}$ was purified by flash chromatography on silica gel (hexane/Et 3 N/EtOAc 3:0.5:9720:0.5:80, $10 \mathrm{mmol}$ scale, $2.55 \mathrm{~g}, 43 \%$ isolated yield). Yellow oil. IR (neat) 743, 1107, 1506, 1626, 2821, 2875, 2924, 3028, 3060, 3361, $3439 \mathrm{~cm}^{-1} .{ }^{1} \mathbf{H}$ NMR (400 MHz, $\left.\mathrm{CDCl}_{3}\right) \delta 3.32$ (s, 3H), 3.45$3.48(\mathrm{~m}, 2 \mathrm{H}), 3.69-3.71(\mathrm{~m}, 2 \mathrm{H}), 3.85-3.87(\mathrm{~m}, 2 \mathrm{H}), 4.19-4.22(\mathrm{~m}, 2 \mathrm{H}), 6.96-7.04(\mathrm{~m}, 3 \mathrm{H}), 7.13(\mathrm{~m}$, 1H), 7.44-7.48 (m, 3H), 7.89-7.91 (m, 2H). $8.48(\mathrm{~s}, 1 \mathrm{H}) .{ }^{13} \mathbf{C ~ N M R}\left(100.5 \mathrm{MHz}, \mathrm{CDCl}_{3}\right) \delta 58.9$, 68.9, 69.6, 70.8, 71.9, 114.0, 120.9, 121.6, 126.3, 128.6, 128.7, 131.2, 136.4, 142.2, 151.1, 161.5 . HRMS-DART $(\mathrm{m} / \mathrm{z}):[\mathrm{M}+\mathrm{H}]^{+}$calcd for $\mathrm{C}_{18} \mathrm{H}_{22} \mathrm{~N}_{1} \mathrm{O}_{3}, 300.1594$; found, 300.1602 .

$(E)-N-\{2-[2-(2-M e t h o x y e t h o x y) e t h o x y] p h e n y l\}-1-(p-t o l y l) m e t h a n i m i n e ~(2 g)$

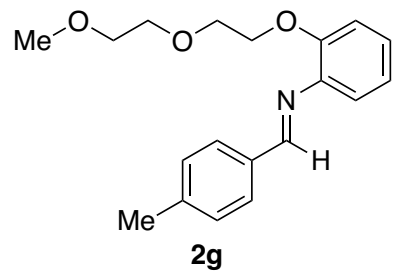

The imine $2 \mathrm{~g}$ was purified by flash chromatography on silica gel (hexane/Et 3 N/EtOAc 3:0.5:9720:0.5:80, $10 \mathrm{mmol} \mathrm{scale,} 997 \mathrm{mg}, 32 \%$ isolated yield). Yellow oil. IR (neat) 745, 815, 1106, 1247, 1488, 1626, 2819, 2874, 2920, 3026, 3057, $3552 \mathrm{~cm}^{-1} .{ }^{1} \mathbf{H}$ NMR (400 MHz, $\left.\mathrm{CDCl}_{3}\right) \delta 2.41(\mathrm{~s}, 3 \mathrm{H})$, $3.33(\mathrm{~s}, 3 \mathrm{H}), 3.46-3.48(\mathrm{~m}, 2 \mathrm{H}), 3.85(\mathrm{t}, J=4.4 \mathrm{~Hz}, 2 \mathrm{H}), 3.83-3.86(\mathrm{~m}, 2 \mathrm{H}), 4.20(\mathrm{t}, J=5.2 \mathrm{~Hz}, 2 \mathrm{H})$, 6.96-7.03 (m, 3H), $7.13(\mathrm{~m}, 1 \mathrm{H}), 7.25-7.27(\mathrm{~m}, 2 \mathrm{H}), 7.78-7.80(\mathrm{~m}, 2 \mathrm{H}) .8 .44(\mathrm{~s}, 1 \mathrm{H}) .{ }^{13} \mathbf{C}$ NMR $\left(100.5 \mathrm{MHz}, \mathrm{CDCl}_{3}\right) \delta 21.6,58.9,69.0,69.7,70.8,71.9,114.1,120.9,121.6,126.2,128.7,129.4$, 133.9, 141.6, 142.5, 151.2, 161.4. HRMS-DART $(\mathrm{m} / \mathrm{z}):[\mathrm{M}+\mathrm{H}]^{+}$calcd for $\mathrm{C}_{19} \mathrm{H}_{24} \mathrm{~N}_{1} \mathrm{O}_{3}, 314.1751$; found, 314.1749 . 


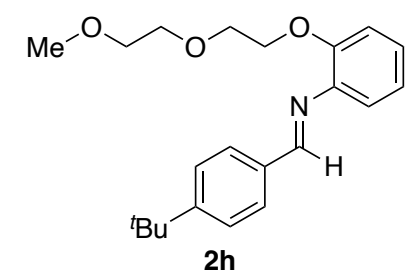

The imne $\mathbf{2 h}$ was purified by flash chromatography on silica gel (hexane/Et ${ }_{3} \mathrm{~N} /$ EtOAc 3:0.5:9720:0.5:80, $10 \mathrm{mmol}$ scale, $515 \mathrm{mg}, 15 \%$ isolated yield). Yellow oil. IR (neat) 748, 1106, 1248, 1487, 1627, 2870, 2960, 3028, 3060, $3564 \mathrm{~cm}^{-1} .{ }^{\mathbf{1}} \mathbf{H}$ NMR (400 MHz, $\left.\mathrm{CDCl}_{3}\right) \delta 1.35(\mathrm{~s}, 9 \mathrm{H}), 3.31(\mathrm{~s}, 3 \mathrm{H})$, 3.45-3.47 (m, 2H), 3.69-3.71 (m, 2H), $3.84(\mathrm{t}, J=5.2 \mathrm{~Hz}, 2 \mathrm{H}), 4.19(\mathrm{t}, J=5.2 \mathrm{~Hz}, 2 \mathrm{H}), 6.95-7.01$ (m, 3H), $7.12(\mathrm{~m}, 1 \mathrm{H}), 7.47-7.49(\mathrm{~m}, 2 \mathrm{H}), 7.82-7.84(\mathrm{~m}, 2 \mathrm{H}), 8.44(\mathrm{~s}, 1 \mathrm{H}) .{ }^{13} \mathrm{C}$ NMR $(100.5 \mathrm{MHz}$, $\left.\mathrm{CDCl}_{3}\right) \delta 31.1,34.9,58.8,68.9,69.6,70.7,71.8,114.1,120.8,121.5,125.5,126.1,128.5,133.8$, 142.5, 151.0, 154.6, 161.2. HRMS-DART $(\mathrm{m} / \mathrm{z})$ : $[\mathrm{M}+\mathrm{H}]^{+}$calcd for $\mathrm{C}_{22} \mathrm{H}_{30} \mathrm{~N}_{1} \mathrm{O}_{3}, 356.2220$; found, 356.2226 .

(E)-N-\{2-[2-(2-Methoxyethoxy)ethoxy]phenyl\}-1-(o-tolyl)methanimine (2i)

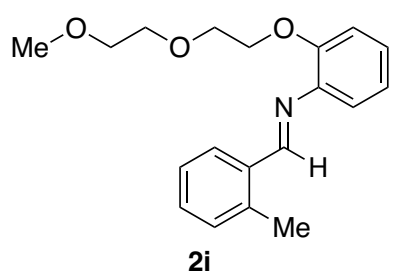

The imine $2 \mathbf{i}$ was purified by flash chromatography on silica gel (hexane/Et 3 N/EtOAc 3:0.5:9720:0.5:80, $10 \mathrm{mmol} \mathrm{scale,} 1.18 \mathrm{~g}, 38 \%$ isolated yield). Yellow oil. IR (neat) 747, 1106, 1246, 1492, 1622, 2820, 2874, 2921, 3024, 3059, $3462 \mathrm{~cm}^{-1} .{ }^{1} \mathbf{H}$ NMR (400 MHz, $\left.\mathrm{CDCl}_{3}\right) \delta 2.57(\mathrm{~s}, 3 \mathrm{H}), 3.31(\mathrm{~s}$, $3 \mathrm{H}), 3.44-3.47(\mathrm{~m}, 2 \mathrm{H}), 3.67-3.69(\mathrm{~m}, 2 \mathrm{H}), 3.84(\mathrm{t}, J=5.2 \mathrm{~Hz}, 2 \mathrm{H}), 4.18-4.21(\mathrm{~m}, 2 \mathrm{H}), 6.96-7.02$ (m, 3H), $7.12(\mathrm{~m}, 1 \mathrm{H}), 7.14(\mathrm{~m}, 1 \mathrm{H}), 7.19-7.35(\mathrm{~m}, 2 \mathrm{H}), 8.08(\mathrm{~m}, 1 \mathrm{H}), 8.77(\mathrm{~s}, 1 \mathrm{H}) .{ }^{13} \mathbf{C}$ NMR $(100.5$ $\left.\mathrm{MHz}, \mathrm{CDCl}_{3}\right) \delta 19.3,58.8,68.7,69.6,70.7,71.8,113.9,121.0,121.5,126.0,126.1,127.8,130.7$, 130.8, 134.3, 138.4, 142.7, 150.9, 160.2. HRMS-DART $(\mathrm{m} / \mathrm{z}):[\mathrm{M}+\mathrm{H}]^{+}$calcd for $\mathrm{C}_{19} \mathrm{H}_{24} \mathrm{~N}_{1} \mathrm{O}_{3}$, 314.1751; found, 314.1758 .

(E)-1-(4-Chlorophenyl)- $N$-(2-(2-(2-methoxyethoxy)ethoxy)phenyl)methanimine (2j)

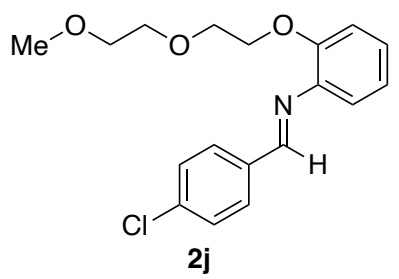

The imine $2 \mathbf{j}$ was purified by flash chromatography on silica gel (hexane/Et 3 N/EtOAc 3:0.5:9720:0.5:80, $10 \mathrm{mmol} \mathrm{scale,} 1.53 \mathrm{~g}, 46 \%$ isolated yield). Yellow oil. IR (neat) 747, 1107, 1490, 1626, 2820, 2874, 2922, 3027, 3062, $3555 \mathrm{~cm}^{-1} .{ }^{1} \mathbf{H}$ NMR (400 MHz, $\left.\mathrm{CDCl}_{3}\right) \delta 3.32(\mathrm{~s}, 3 \mathrm{H}), 3.46-3.49(\mathrm{~m}$, 2H), 3.67-3.70 (m, 2H), 3.83-3.85 (m, 2H), 4.19 (t, J=5.2 Hz, 2H), 6.95-7.03 (m, 3H), 7.14 (m, 
1H), 7.40-7.42 (m, 2H), 7.82-7.84 (m, 2H), 8.46 (s, 1H). ${ }^{13} \mathbf{C}$ NMR (100.5 MHz, $\left.\mathrm{CDCl}_{3}\right) \delta 58.8$, 68.7, 69.5, 70.7, 71.8, 113.9, 121.0, 121.5, 126.5, 128.8, 129.7, 134.9, 137.0, 141.6, 151.0, 159.9. HRMS-DART (m/z): [M+H $]^{+}$calcd for $\mathrm{C}_{18} \mathrm{H}_{21} \mathrm{Cl}_{1} \mathrm{~N}_{1} \mathrm{O}_{3}, 334.1204$; found, 334.1205.

(E)-N-\{2-[2-(2-Methoxyethoxy)ethoxy]phenyl\}-1-(naphthalen-2-yl)methanimine (2k)

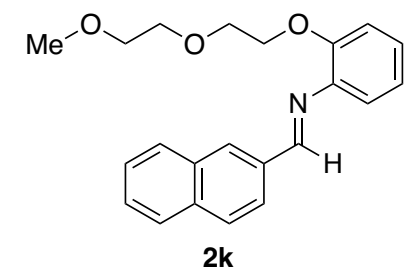

The imine $2 \mathbf{k}$ was purified by flash chromatography on silica gel (hexane/Et 3 N/EtOAc 3:0.5:9720:0.5:80, $10 \mathrm{mmol} \mathrm{scale,} 1.00 \mathrm{~g}, 32 \%$ isolated yield). Yellow oil. IR (neat) 746, 1114, 1244, 1491, $1619,2822,2874,2922,3057,3564 \mathrm{~cm}^{-1} .{ }^{1} \mathbf{H}$ NMR (600 MHz, $\left.\mathrm{CDCl}_{3}\right) \delta 3.30(\mathrm{~s}, 3 \mathrm{H}), 3.46-3.47(\mathrm{~m}$, 2H), 3.71-3.72 (m, 2H), $3.88(\mathrm{t}, J=5.4 \mathrm{~Hz}, 2 \mathrm{H}), 4.24(\mathrm{t}, J=5.4 \mathrm{~Hz}, 2 \mathrm{H}), 7.00-7.03(\mathrm{~m}, 2 \mathrm{H}), 7.09$ (m, 1H), $7.17(\mathrm{~m}, 1 \mathrm{H}), 7.52-7.57(\mathrm{~m}, 2 \mathrm{H}), 7.87-7.93(\mathrm{~m}, 3 \mathrm{H}), 8.17-8.19(\mathrm{~m}, 2 \mathrm{H}), 8.66(\mathrm{~s}, 1 \mathrm{H}) .{ }^{13} \mathrm{C}$ NMR $\left(150.9 \mathrm{MHz}, \mathrm{CDCl}_{3}\right) \delta 58.9,69.0,69.7,70.8,71.9,114.1,121.0,121.6,123.9,126.4,126.5$, 127.4, 127.9, 128.5, 128.7, 131.0, 133.1, 134.2, 134.9, 142.3, 151.2, 161.5. HRMS-DART (m/z): $[\mathrm{M}+\mathrm{H}]^{+}$calcd for $\mathrm{C}_{22} \mathrm{H}_{24} \mathrm{~N}_{1} \mathrm{O}_{3}, 350.1751$; found, 350.1754 .

(E)-N-\{2-[2-(2-Methoxyethoxy)ethoxy]phenyl\}-1-(thiophen-2-yl)methanimine (2I)

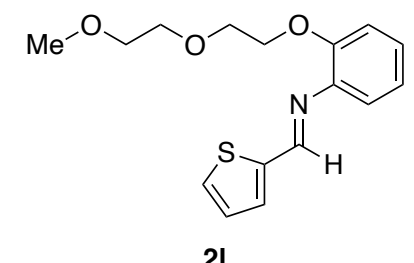

The imine 21 was purified by flash chromatography on silica gel (hexane/Et 3 N/EtOAc 3:0.5:9720:0.5:80, $10 \mathrm{mmol}$ scale, $1.70 \mathrm{~g}, 56 \%$ isolated yield). Yellow oil. IR (neat) 748, 1115, 1247, 1426, 1488, 1614, 2822, 2875, 2923, 3071, $3543 \mathrm{~cm}^{-1} .{ }^{1} \mathbf{H}$ NMR (600 MHz, $\left.\mathrm{CDCl}_{3}\right) \delta 3.35$ (s, 3H), 3.50$3.52(\mathrm{~m}, 2 \mathrm{H}), 3.74-3.76(\mathrm{~m}, 2 \mathrm{H}), 3.88(\mathrm{t}, J=4.8 \mathrm{~Hz}, 2 \mathrm{H}), 4.20(\mathrm{t}, J=4.8 \mathrm{~Hz}, 2 \mathrm{H}), 6.95-6.99(\mathrm{~m}, 2 \mathrm{H})$, $7.05(\mathrm{~m}, 1 \mathrm{H}), 7.12-7.15(\mathrm{~m}, 2 \mathrm{H}), 7.46(\mathrm{~m}, 1 \mathrm{H}), 7.49(\mathrm{~m}, 1 \mathrm{H}), 8.63(\mathrm{~s}, 1 \mathrm{H}) .{ }^{13} \mathbf{C}$ NMR $(150.9 \mathrm{MHz}$, $\left.\mathrm{CDCl}_{3}\right) \delta 58.9,69.0,69.6,70.9,71.9,114.0,121.4,121.5,126.4,127.5,130.0,131.7,141.3,143.3$, 151.2, 154.3. HRMS-DART $(m / z)$ : $[\mathrm{M}+\mathrm{H}]^{+}$calcd for $\mathrm{C}_{16} \mathrm{H}_{20} \mathrm{~N}_{1} \mathrm{O}_{3} \mathrm{~S}_{1}, 306.1158$; found, 306.1166 .

\section{(E)-N-\{2-[2-(2-Methoxyethoxy)ethoxy]phenyl\}-1-phenylethan-1-imine (2m)}

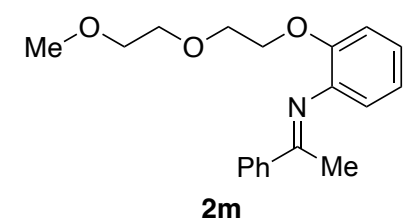

The ketimine $\mathbf{2 m}$ was purified by flash chromatography on silica gel (hexane/Et ${ }_{3} \mathrm{~N} / \mathrm{EtOAc}$ 3:0.5:97-20:0.5:80, $7 \mathrm{mmol}$ scale, $1.11 \mathrm{~g}, 56 \%$ isolated yield). The mixture of $E / Z$ was obtained and used for the coupling reaction. Yellow oil. IR (neat) 745, 1110, 1241, 1446, 1487, 1634, 2874, 2921, 
3024, 3057, $3662 \mathrm{~cm}^{-1} .{ }^{1} \mathbf{H}$ NMR $\left(600 \mathrm{MHz}, \mathrm{CDCl}_{3}\right) \delta 1.78(\mathrm{~s}, 0.43 \times 3 \mathrm{H}), 2.17(\mathrm{~s}, 0.57 \times 3 \mathrm{H}), 3.25$ $(\mathrm{s}, 0.43 \times 3 \mathrm{H}), 3.29(\mathrm{~s}, 0.57 \times 3 \mathrm{H}), 3.36-3.38(\mathrm{~m}, 2 \mathrm{H}), 3.57-3.59(\mathrm{~m}, 2 \mathrm{H}), 3.73-3.77(\mathrm{~m}, 2 \mathrm{H}), 4.07-$ $4.12(\mathrm{~m}, 2 \mathrm{H}), 6.45(\mathrm{~m}, 0.43 \times 1 \mathrm{H}), 6.80(\mathrm{~m}, 0.57 \times 1 \mathrm{H}), 6.86-7.04(\mathrm{~m}, 3 \mathrm{H}), 7.28(\mathrm{~m}, 1 \mathrm{H}), 7.41-7.47$ (m, 2H), 7.98-7.99 (m, 2H). ${ }^{13} \mathbf{C}$ NMR (100.5 MHz, $\left.\mathrm{CDCl}_{3}\right) \delta 17.8,18.5,58.79,58.81,68.2,68.3$, 69.6, 70.7, 71.8, 113.2, 113.4, 120.8, 121.0, 121.2, 121.4, 123.4, 124.0, 124.3, 125.7, 127.1, 127.6, $128.1,128.2,128.3,130.2,130.4,138.7,139.4,140.5,141.0,141.2,141.5,147.6,147.8,167.1,168.0$. HRMS-DART $(\mathrm{m} / \mathrm{z})$ : $[\mathrm{M}+\mathrm{H}]^{+}$calcd for $\mathrm{C}_{19} \mathrm{H}_{24} \mathrm{~N}_{1} \mathrm{O}_{3}, 314.1751$; found, 314.1757 .

- Procedure for Copper-Catalyzed Enantioselective Reductive Cross-Coupling between Aldehydes and Imines or Ketimines
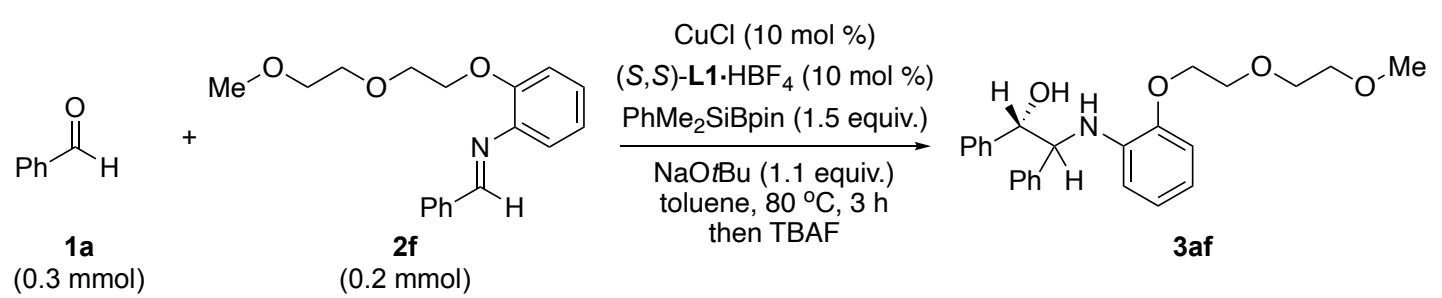

Table 1, entry 11 is representative. $\mathrm{CuCl}(2.0 \mathrm{mg}, 0.02 \mathrm{mmol}),(S, S)-\mathbf{L 1} \cdot \mathrm{HBF}_{4}(13.4 \mathrm{mg}, 0.02$ $\mathrm{mmol})$ and $\mathrm{NaOSiMe}_{3}(4.5 \mathrm{mg}, 0.04 \mathrm{mmol})$ were placed in a vial containing a magnetic stirring bar. The vial was sealed with a Teflon ${ }^{\circledR}$-coated silicon rubber septum, and then the vial was evacuated and filled with argon. Cyclooctane $(0.5 \mathrm{~mL})$ was added to the vial, and then the mixture was stirred at room temperature for $10 \mathrm{~min}$. Next, $\mathrm{PhMe} 2 \mathrm{SiB}$ (pin) $(81.8 \mu \mathrm{L}, 0.3 \mathrm{mmol})$ and benzaldehyde (1a) $(30.4$ $\mu \mathrm{L}, 0.3 \mathrm{mmol}$ ) were added, and then the mixture was stirred at room temperature for $20 \mathrm{~min}$. Then, cyclooctane $(0.5 \mathrm{~mL})$ was added to the vial, and $\mathrm{NaOSiMe}_{3}(20.2 \mathrm{mg}, 0.18 \mathrm{mmol})$, imine (2f) (59.9 $\mathrm{mg}, 0.2 \mathrm{mmol}$ ) was added. After $3 \mathrm{~h}$ stirring at $40{ }^{\circ} \mathrm{C}$ with oil bath, the reaction mixture was treated with TBAF $(600 \mu \mathrm{L})$ and stirred for $1 \mathrm{~h}$. After addition of water to the reaction, the reaction mixture was extracted with ethyl acetate (3 times), washed with brine and dried over sodium sulfate. After filtration, the resulting solution was evaporated under reduced pressure. purification by flash column chromatography (5:0.5:95-30:0.5:70, hexane/Et $\left.{ }_{3} \mathrm{~N} / \mathrm{EtOAc}\right)$ on silica gel gave the 3af (66.4 mg, 81\%) as a yellow oil. 


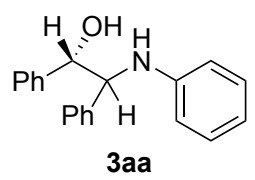

The spectrum and HRMS data of product 3 aa were consistent with the literature. ${ }^{3}$ The product $\mathbf{3 a a}$ was purified by flash chromatography on silica gel (hexane/Et ${ }_{3} \mathrm{~N} / \mathrm{EtOAc}, 3: 0.5: 97-20: 0.5: 80$ ) (Table $1 ; .55 .6 \mathrm{mg}, 0.192 \mathrm{mmol}, 96 \%$ isolated yield). The diastereomeric ratio (1:1.36) was determined by ${ }^{1} \mathrm{H}$ NMR. The ee value (24\% ee/22\% ee) was determined by chiral HPLC analysis [CHIRALCEL ${ }^{\circledR}$ OJ-3 column, $4.6 \mathrm{~mm} \times 250 \mathrm{~mm}$, Daicel Chemical Industries, hexane $/ 2$-propanol $=93: 7,1.0 \mathrm{~mL} / \mathrm{min}$, $40{ }^{\circ} \mathrm{C}, 250 \mathrm{~nm} \mathrm{UV}$ detector, $t_{\mathrm{R}}=21.0 \min \left(\right.$ major $\left._{\mathrm{B}}\right), 24.3 \min \left(\right.$ minor $\left._{\mathrm{A}}\right), 30.4 \min \left(\right.$ major $\left._{\mathrm{A}}\right), 44.8 \mathrm{~min}$ $\left(\right.$ minor $\left.\left._{\mathrm{B}}\right)\right]$. The absolute configuration of 3aa was assigned by consideration of the stereochemical pathway.

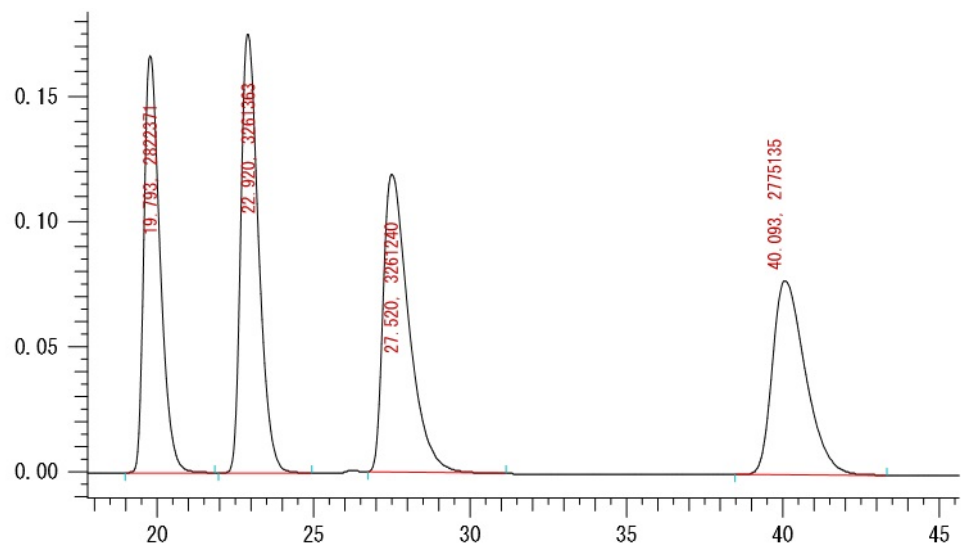

$$
r a c-3 a a
$$$$
\text { No } \quad \mathrm{rt}(\mathrm{min}) \text { area } \quad \text { area }(\%)
$$$$
\begin{array}{llll}
1 & 19.79 & 2822371 & 23.287
\end{array}
$$$$
\begin{array}{llll}
2 & 22.92 & 3261363 & 26.909
\end{array}
$$

$\begin{array}{llll}3 & 27.52 & 3261240 & 26.908\end{array}$

$\begin{array}{llll}4 & 40.09 & 2775135 & 22.897\end{array}$

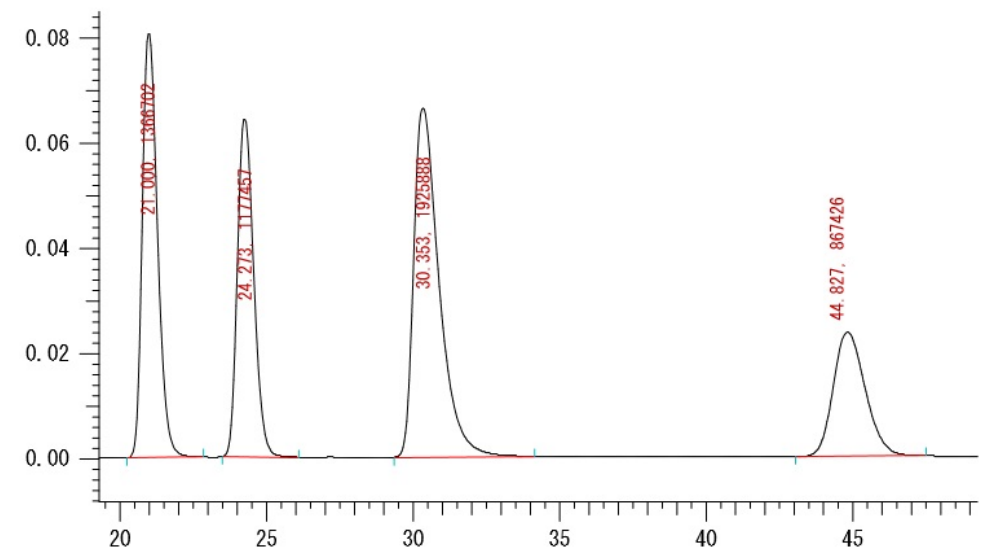

(1S)-3aa

No $\mathrm{rt}(\mathrm{min})$ area $\quad$ area $(\%)$

$\begin{array}{llll}1 & 21.00 & 1366702 & 25.606\end{array}$

$\begin{array}{llll}2 & 24.27 & 1177457 & 22.060\end{array}$

$\begin{array}{llll}3 & 30.35 & 1925888 & 36.082\end{array}$

$\begin{array}{llll}4 & 44.83 & 867426 & 16.252\end{array}$ 


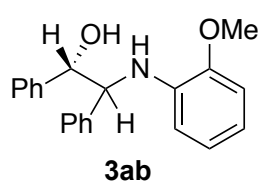

The spectrum and HRMS data of product 3ab were consistent with the literature. ${ }^{4}$ The product $\mathbf{3 a b}$ was purified by flash chromatography on silica gel (hexane/Et ${ }_{3} \mathrm{~N} /$ EtOAc 3:0.5:97-20:0.5:80) (Table $1 ; .59 .3 \mathrm{mg}, 0.186 \mathrm{mmol}, 93 \%$ isolated yield). The diastereomeric ratio (1:1.86) was determined by ${ }^{1} \mathrm{H}$ NMR. The ee value $\left(58 \%\right.$ ee/51\% ee) was determined by chiral HPLC analysis [CHIRALCEL ${ }^{\circledR}$ OD-3 column, $4.6 \mathrm{~mm} \times 250 \mathrm{~mm}$, Daicel Chemical Industries, hexane $/ 2$-propanol = 95:5, $1.0 \mathrm{~mL} / \mathrm{min}$, $40{ }^{\circ} \mathrm{C}, 250 \mathrm{~nm} \mathrm{UV}$ detector, $\mathrm{t}_{\mathrm{R}}=16.6 \min \left(\operatorname{minor}_{\mathrm{B}}\right), 27.9 \min \left(\right.$ major $\left._{\mathrm{A}}\right), 42.6 \min \left(\operatorname{minor}_{\mathrm{A}}\right), 59.7 \mathrm{~min}$ (major $)$ ]. The absolute configuration of $\mathbf{3 a b}$ was assigned by consideration of the stereochemical pathway.

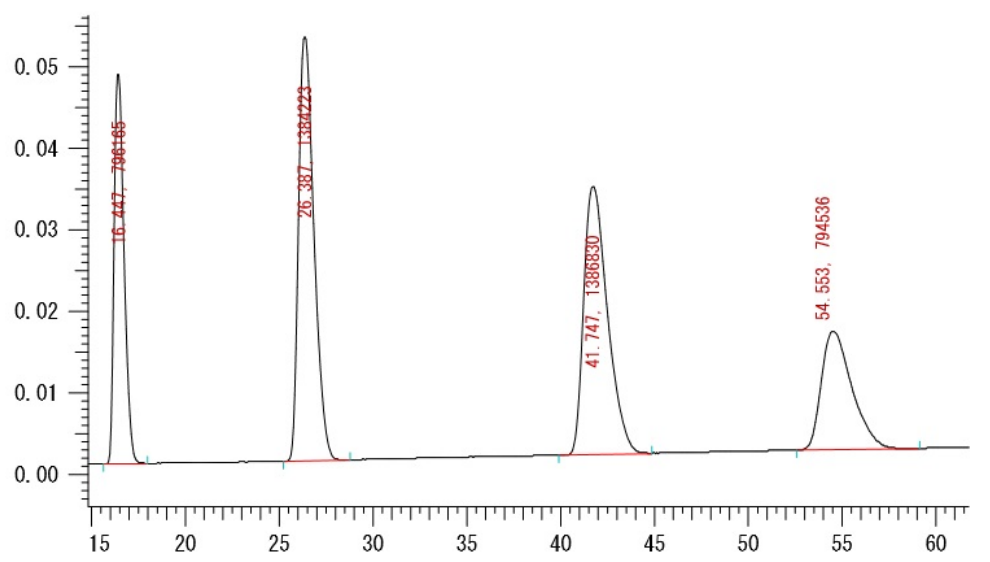

$r a c-3 \mathbf{a b}$

No $\mathrm{rt}(\mathrm{min})$ area $\quad$ area $(\%)$

$\begin{array}{llll}1 & 16.45 & 796165 & 18.253\end{array}$

$2 \quad 26.39 \quad 1384223 \quad 31.735$

$3 \quad 41.75 \quad 1386830 \quad 31.795$

$4 \quad 54.55 \quad 794536 \quad 18.216$

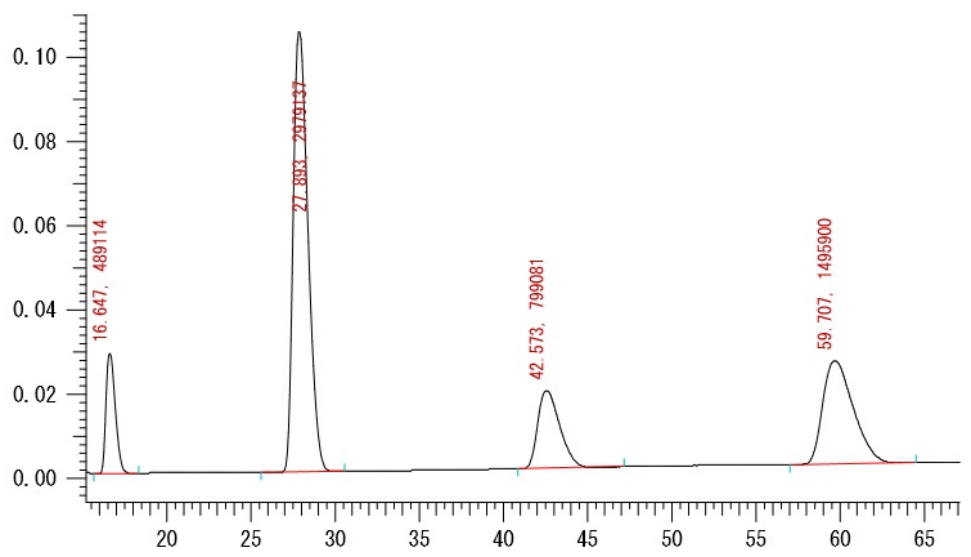

(1S)-3ab

No $\mathrm{rt}(\mathrm{min})$ area $\quad$ area $(\%)$

$\begin{array}{llll}1 & 16.65 & 489114 & 8.487\end{array}$

$\begin{array}{llll}2 & 27.89 & 2979137 & 51.692\end{array}$

$\begin{array}{llll}3 & 42.57 & 799081 & 13.865\end{array}$

$\begin{array}{llll}4 & 59.71 & 1495900 & 25.956\end{array}$ 


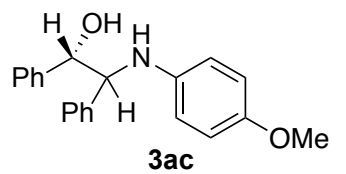

The spectrum and HRMS data of product 3ac were consistent with the literature. ${ }^{3}$ The product 3ac was purified by flash chromatography on silica gel (hexane/Et ${ }_{3} \mathrm{~N} /$ EtOAc 3:0.5:97-20:0.5:80) (Table 1; $.57 .0 \mathrm{mg}, 0.178 \mathrm{mmol}, 89 \%$ isolated yield). The diastereomeric ratio is $1: 1.20$ determined by ${ }^{1} \mathrm{H}$ NMR. The ee value $\left(26 \%\right.$ ee/26\% ee) was determined by chiral HPLC analysis [CHIRALCEL ${ }^{\circledR}$ OJ3 column, $4.6 \mathrm{~mm} \times 250 \mathrm{~mm}$, Daicel Chemical Industries, hexane $/ 2$-propanol $=97: 3,1.0 \mathrm{~mL} / \mathrm{min}$, $40{ }^{\circ} \mathrm{C}, 250 \mathrm{~nm} \mathrm{UV}$ detector, $\mathrm{t}_{\mathrm{R}}=79.8 \min \left(\right.$ major $\left._{\mathrm{B}}\right), 84.8 \min \left(\right.$ (minor $\left._{\mathrm{B}}\right), 111.0 \min \left(\right.$ minor $\left._{\mathrm{A}}\right), 173.5$ $\min \left(\right.$ major $\left.\left._{\mathrm{A}}\right)\right]$. The absolute configuration of 3ac was assigned by consideration of the stereochemical pathway.

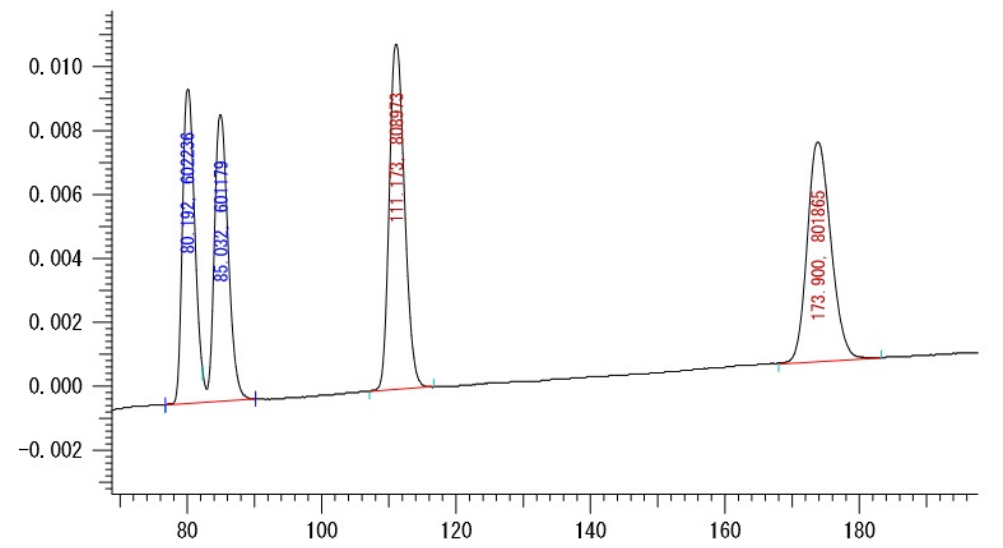

rac-3ac

No rt (min) area area (\%)

$\begin{array}{llll}1 & 80.19 & 602236 & 21.399\end{array}$

$\begin{array}{llll}2 & 85.03 & 601179 & 21.362\end{array}$

$3 \quad 111.17 \quad 808973 \quad 28.746$

$4 \quad 173.90 \quad 801865 \quad 28.493$

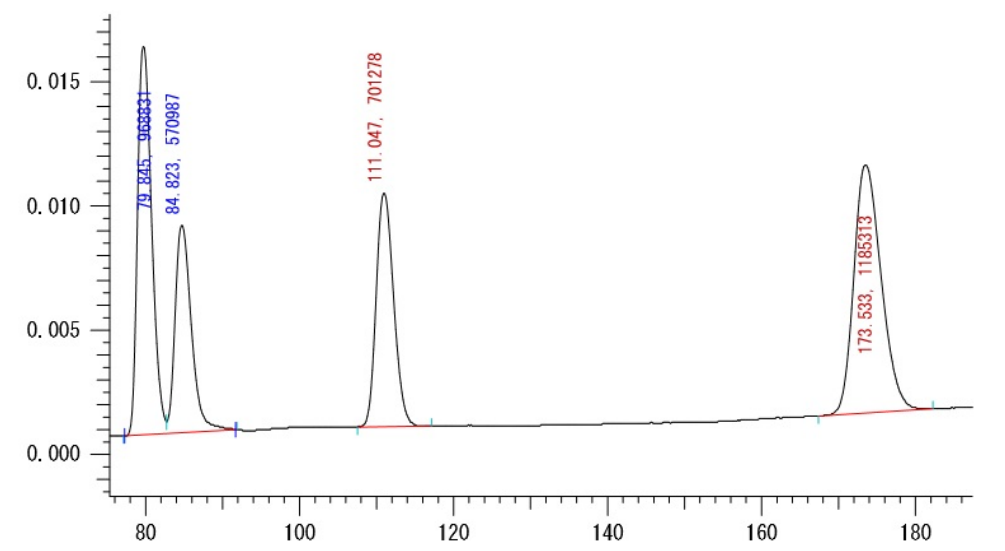

(1S)-3ac

No $\mathrm{rt}(\mathrm{min})$ area area (\%)

$1 \quad 79.85 \quad 968831 \quad 28.275$

$\begin{array}{llll}2 & 84.82 & 570987 & 16.664\end{array}$

$\begin{array}{llll}3 & 111.05 & 701278 & 20.467\end{array}$

$\begin{array}{llll}4 & 173.53 & 1185313 & 34.593\end{array}$ 


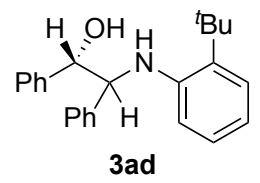

The product 3ad was purified by flash chromatography on silica gel (hexane/Et $3 \mathrm{~N} / \mathrm{EtOAc}$ 3:0.5:97-20:0.5:80). (Table 1; .39.9 mg, $0.115 \mathrm{mmol}, 58 \%$ isolated yield). The diastereomeric ratio (1:1.94) was determined by ${ }^{1} \mathrm{H}$ NMR. Yellow oil. IR (neat) 699, 744, 1052, 1307, 1448, 1506, 1599, 2872, 2911, 2965, 3029, 3061, 3445, $3542 \mathrm{~cm}^{-1} .{ }^{1} \mathbf{H}$ NMR $\left(600 \mathrm{MHz}, \mathrm{CDCl}_{3}\right) \delta 1.38(\mathrm{~s}, 0.34 \times 9 \mathrm{H})$, $1.44(\mathrm{~s}, 0.66 \times 9 \mathrm{H}), 2.23(\mathrm{~d}, J=1.2 \mathrm{~Hz}, 0.66 \times 1 \mathrm{H}), 2.26(\mathrm{~d}, J=4.8 \mathrm{~Hz}, 0.34 \times 1 \mathrm{H}), 4.68-4.76(\mathrm{~m}$, $1.5 \mathrm{H}), 5.01-5.09(\mathrm{~m}, 1.5 \mathrm{H}), 6.29(\mathrm{~d}, J=7.8 \mathrm{~Hz}, 0.66 \times 1 \mathrm{H}), 6.35(\mathrm{~d}, J=7.8 \mathrm{~Hz}, 0.34 \times 1 \mathrm{H}), 6.59$ $(\mathrm{m}, 1 \mathrm{H}), 6.87(\mathrm{~m}, 1 \mathrm{H}), 7.13-7.37(\mathrm{~m}, 11 \mathrm{H}) .{ }^{13} \mathbf{C} \mathbf{N M R}\left(150.9 \mathrm{MHz}, \mathrm{CDCl}_{3}\right) \delta 29.7,29.9,34.1,34.2$, 64.1, 64.4, 77.8, 78.0, 112.8, 112.9, 116.9, 117.2, 126.1, 126.1, 126.3, 126.8, 126.8, 126.82, 127.2, $127.5,127.5,127.8,128.2,128.3,128.3,128.4,128.7(\times 2 \mathrm{C}), 133.6(\times 2 \mathrm{C}), 139.2,139.8,140.5$, 140.7, 144.4, 144.7. HRMS-DART $(\mathrm{m} / \mathrm{z})$ : $[\mathrm{M}+\mathrm{H}]^{+}$calcd for $\mathrm{C}_{24} \mathrm{H}_{28} \mathrm{~N}_{1} \mathrm{O}_{1}, 346.2165$; found, 346.2165. The ee value $\left(12 \%\right.$ ee/18\% ee) was determined by chiral HPLC analysis [CHIRALCEL ${ }^{\circledR}$ AD-3 column, $4.6 \mathrm{~mm} \times 250 \mathrm{~mm}$, Daicel Chemical Industries, hexane/2-propanol = 97:3, 1.0

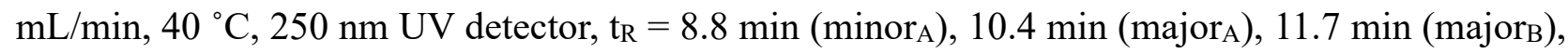
$14.7 \min \left(\right.$ minor $\left.\left._{\mathrm{B}}\right)\right]$. The absolute configuration of $\mathbf{3 a d}$ was assigned by consideration of the stereochemical pathway.

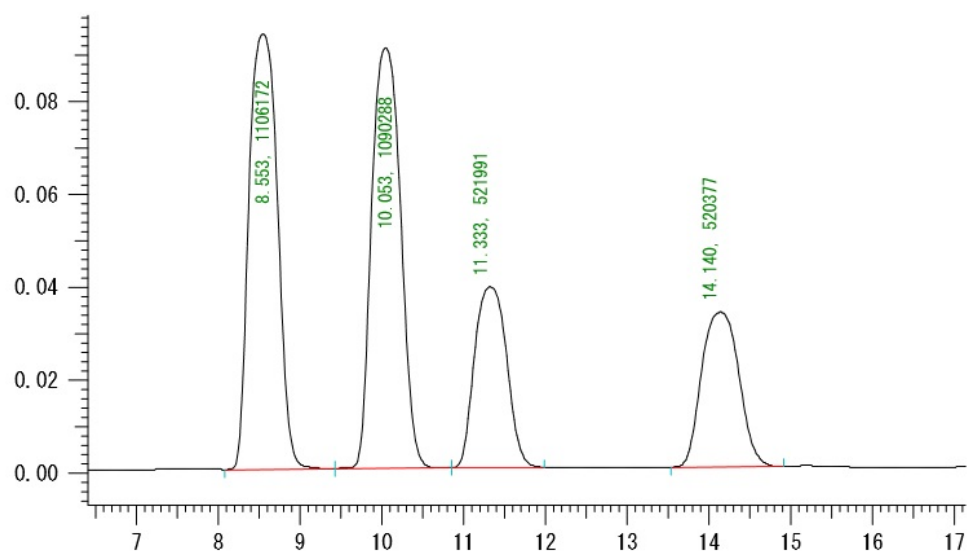

rac-3ad

$\begin{array}{llll}\text { No } & \text { rt (min) } & \text { area } & \text { area }(\%) \\ 1 & 8.55 & 1106172 & 34.153 \\ 2 & 10.05 & 1090288 & 33.663 \\ 3 & 11.33 & 521991 & 16.117 \\ 4 & 14.14 & 520337 & 16.067\end{array}$

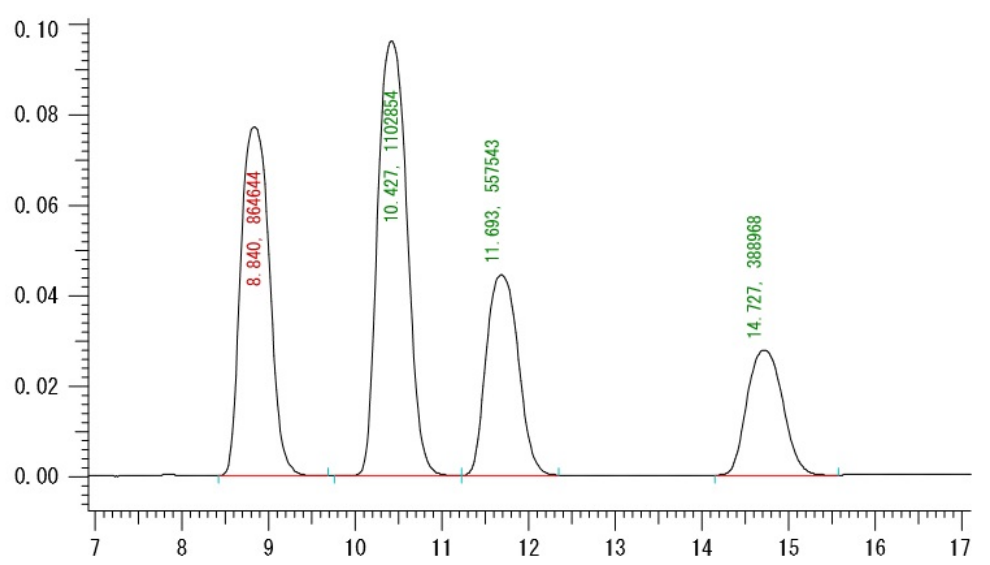

(1S)-3ad

No $\mathrm{rt}(\mathrm{min})$ area $\quad$ area $(\%)$

$1 \quad 8.84 \quad 864644 \quad 29.672$

$2 \quad 10.43 \quad 1102854 \quad 37.847$

$3 \quad 11.69 \quad 557543 \quad 19.133$

$\begin{array}{llll}4 & 14.73 & 388968 & 13.348\end{array}$ 


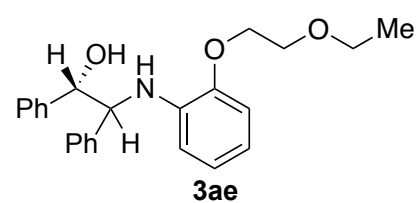

The product 3ae was purified by flash chromatography on silica gel (hexane/Et 3 N/EtOAc 5:0.5:9530:0.5:70). (Table 1; .61.6 mg, $0.163 \mathrm{mmol}, 82 \%$ isolated yield). The diastereomeric ratio (1:1.22) was determined by ${ }^{1} \mathrm{H}$ NMR. Yellow oil. IR (neat) 701, 737, 1050, 1125, 1215, 1246, 1452, 1511, 1601, 2872, 2928, 2974, 3029, 3061, $3418 \mathrm{~cm}^{-1} .{ }^{\mathbf{1}} \mathbf{H}$ NMR $\left(600 \mathrm{MHz}, \mathrm{CDCl}_{3}\right) \delta 1.26(\mathrm{t}, J=6.6$ $\mathrm{Hz}, 0.55 \times 3 \mathrm{H}), 1.29(\mathrm{t}, J=6.6 \mathrm{~Hz}, 0.45 \times 3 \mathrm{H}), 2.63($ brs, $0.55 \times 1 \mathrm{H}), 2.99$ (brs, $0.45 \times 1 \mathrm{H}), 3.60$ $3.68(\mathrm{~m}, 2 \mathrm{H}), 3.75-3.85(\mathrm{~m}, 2 \mathrm{H}), 4.08-4.16(\mathrm{~m}, 2 \mathrm{H}), 4.50(\mathrm{~d}, J=6.0 \mathrm{~Hz}, 0.45 \times 1 \mathrm{H}), 4.68(\mathrm{~d}, J=4.8$ $\mathrm{Hz}, 0.55 \times 1 \mathrm{H}), 4.86(\mathrm{~d}, J=6.6 \mathrm{~Hz}, 0.45 \times 1 \mathrm{H}), 5.06(\mathrm{t}, J=4.8 \mathrm{~Hz}, 0.55 \times 1 \mathrm{H}), 5.09(\mathrm{brs}, 0.55 \times 1 \mathrm{H})$, 5.34 (brs, $0.45 \times 1 \mathrm{H}), 6.39(\mathrm{t}, J=7.8 \mathrm{~Hz}, 1 \mathrm{H}), 6.58(\mathrm{t}, J=7.8 \mathrm{~Hz}, 1 \mathrm{H}), 6.68(\mathrm{~m}, 1 \mathrm{H}), 6.76(\mathrm{~m}, 1 \mathrm{H})$, 7.10-7.26 (m, 10H). ${ }^{13} \mathrm{C}$ NMR (150.9 MHz, $\left.\mathrm{CDCl}_{3}\right) \delta 15.2,15.2,63.7,65.1,66.8(\times 2 \mathrm{C}), 68.3,68.8$, 69.1, 69.1, 77.1, 78.2, 111.7, 111.8, 112.0, 112.6, 117.0, 117.1, 121.7, 121.9, 126.6, 126.7, 127.3, $127.4,127.7,127.7,127.7,128.0$ ( $\times 2 \mathrm{C}), 128.0,128.2,128.3,137.3,138.0,138.9,140.1,140.2,140.6$, 146.4, 146.5. HRMS-DART $(\mathrm{m} / \mathrm{z}):[\mathrm{M}+\mathrm{H}]^{+}$calcd for $\mathrm{C}_{24} \mathrm{H}_{28} \mathrm{~N}_{1} \mathrm{O}_{3}, 378.2064$; found, 378.2070. The ee value $\left(71 \%\right.$ ee/72\% ee) was determined by chiral HPLC analysis [CHIRALCEL ${ }^{\circledR}$ AD-3+AD-H column, $4.6 \mathrm{~mm} \times 250 \mathrm{~mm}$, Daicel Chemical Industries, hexane $/ 2$-propanol $=85: 15,1.0 \mathrm{~mL} / \mathrm{min}, 40$ ${ }^{\circ} \mathrm{C}, 250 \mathrm{~nm}$ UV detector, $\mathrm{t}_{\mathrm{R}}=18.5 \min \left(\operatorname{minor}_{\mathrm{A}}\right), 19.1 \min \left(\right.$ major $\left._{\mathrm{A}}\right), 21.1 \min \left(\operatorname{minor}_{\mathrm{B}}\right), 26.4 \min$ (major $\left.)_{\mathrm{B}}\right)$. The absolute configuration of 3ae was assigned by consideration of the stereochemical pathway.

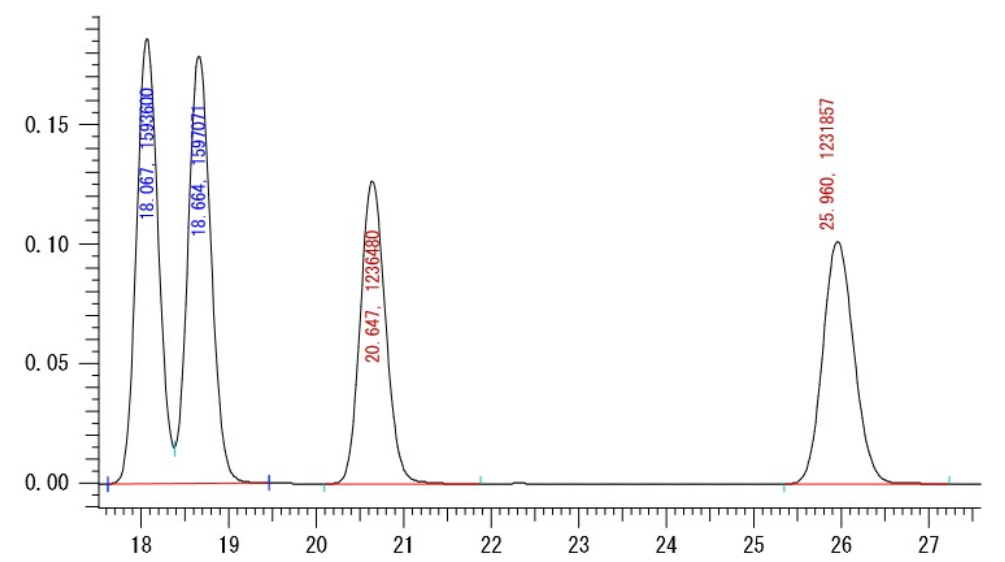

\begin{tabular}{llll}
\multicolumn{2}{l}{$r a c-3 a e$} \\
No & rt (min) & area & area (\%) \\
1 & 18.07 & 1593600 & 28.160 \\
2 & 18.66 & 1597071 & 28.222 \\
3 & 20.65 & 1236480 & 21.850 \\
4 & 25.96 & 1231857 & 21.768
\end{tabular}

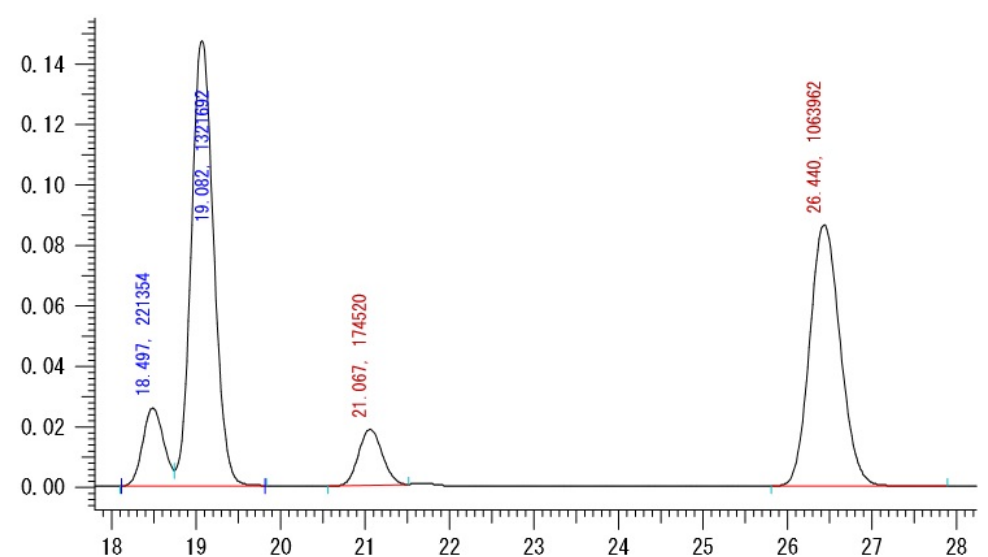

(1S)-3ae

$\begin{array}{llll}\text { No } & \text { rt (min) } & \text { area } & \text { area }(\%) \\ 1 & 18.50 & 221354 & 7.958 \\ 2 & 19.08 & 1321692 & 47.517 \\ 3 & 21.07 & 174520 & 6.274 \\ 4 & 26.44 & 1063962 & 38.251\end{array}$




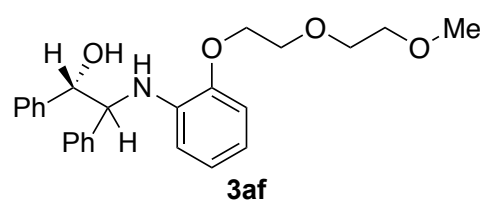

The product 3af was purified by flash chromatography on silica gel (5:0.5:95-30:0.5:70, hexane/Et $3 \mathrm{~N} / \mathrm{EtOAc}$ ) (Table 1; .66.4 $\mathrm{mg}, 0.162 \mathrm{mmol}, 81 \%$ isolated yield). The diastereomeric ratio (1:1) was determined by ${ }^{1} \mathrm{H}$ NMR. Yellow oil. IR (neat) 702, 1051, 1128, 1214, 1452, 1511, 1601, $2877,3028,3061,3413 \mathrm{~cm}^{-1} .{ }^{1} \mathbf{H}$ NMR $\left(400 \mathrm{MHz} \mathrm{CDCl}_{3}\right) \delta 2.98(\mathrm{~d}, J=5.6 \mathrm{~Hz}, 0.5 \times 1 \mathrm{H}), 3.28(\mathrm{~d}$, $J=2.8 \mathrm{~Hz}, 0.5 \times 1 \mathrm{H}), 3.37(\mathrm{~s}, 0.5 \times 3 \mathrm{H}), 3.38(\mathrm{~s}, 0.5 \times 3 \mathrm{H}), 3.58-3.63(\mathrm{~m}, 2 \mathrm{H}), 3.73-3.79(\mathrm{~m}, 2 \mathrm{H})$, 3.84-3.91 (m, 2H), 4.10-4.21 (m, 2H), $4.46(\mathrm{~d}, J=6.8 \mathrm{~Hz}, 0.5 \times 1 \mathrm{H}), 4.66(\mathrm{brs}, 0.5 \times 1 \mathrm{H}), 4.84(\mathrm{~m}$, $0.5 \times 1 \mathrm{H}), 5.10(\mathrm{t}, J=5.2 \mathrm{~Hz}, 0.5 \times 1 \mathrm{H}), 5.23(\mathrm{brs}, 0.5 \times 1 \mathrm{H}), 5.44(\mathrm{brs}, 0.5 \times 1 \mathrm{H}), 6.37(\mathrm{~m}, 1 \mathrm{H}), 6.57$ $(\mathrm{m}, 1 \mathrm{H}), 6.68(\mathrm{~m}, 1 \mathrm{H}), 6.77(\mathrm{~m}, 1 \mathrm{H}), 7.10-7.26(\mathrm{~m}, 10 \mathrm{H}) .{ }^{13} \mathbf{C ~ N M R}\left(100.5 \mathrm{MHz}, \mathrm{CDCl}_{3}\right) \delta 59.0,59.1$, 63.7, 65.1, 68.6, 69.0, 69.9, 70.0, 70.65, 70.69, $72.0(\times 2 \mathrm{C}), 76.9,78.2,111.8,112.1,112.2,112.8$, $116.9,117.0,121.9,122.1,126.6,126.8,127.1,127.3,127.4,127.6,127.9,128.0,128.0,128.1,128.2$, $128.3,137.5,138.2,138.7,140.3,140.4,140.8,146.2,146.4$. HRMS-DART $(\mathrm{m} / z):[\mathrm{M}+\mathrm{H}]^{+}$calcd for $\mathrm{C}_{25} \mathrm{H}_{30} \mathrm{~N}_{1} \mathrm{O}_{4}, 408.2169$; found, 408.2165. [ $\left.\alpha\right]_{\mathrm{D}}{ }^{28}+6.5\left(c 1.06, \mathrm{CHCl}_{3}\right)$. The ee value $(89 \%$ ee/89\% ee) was determined by chiral HPLC analysis [CHIRALCEL ${ }^{\circledR}$ AD-3 column, $4.6 \mathrm{~mm} \times 250 \mathrm{~mm}$, Daicel Chemical Industries, hexane/2-propanol $=90: 10,1.0 \mathrm{~mL} / \mathrm{min}, 40{ }^{\circ} \mathrm{C}, 250 \mathrm{~nm} \mathrm{UV}$ detector, $\mathrm{t}_{\mathrm{R}}$ $=17.3 \min \left(\operatorname{minor}_{\mathrm{A}}\right), 18.9 \min \left(\right.$ major $\left._{\mathrm{A}}\right), 22.4 \min \left(\right.$ minor $\left._{\mathrm{B}}\right), 24.9 \min \left(\right.$ major $\left.\left._{\mathrm{B}}\right)\right]$. The absolute configuration of $\mathbf{3 a f}$ was detemined by the derivatization to $\mathbf{5}$ af and 5af'.

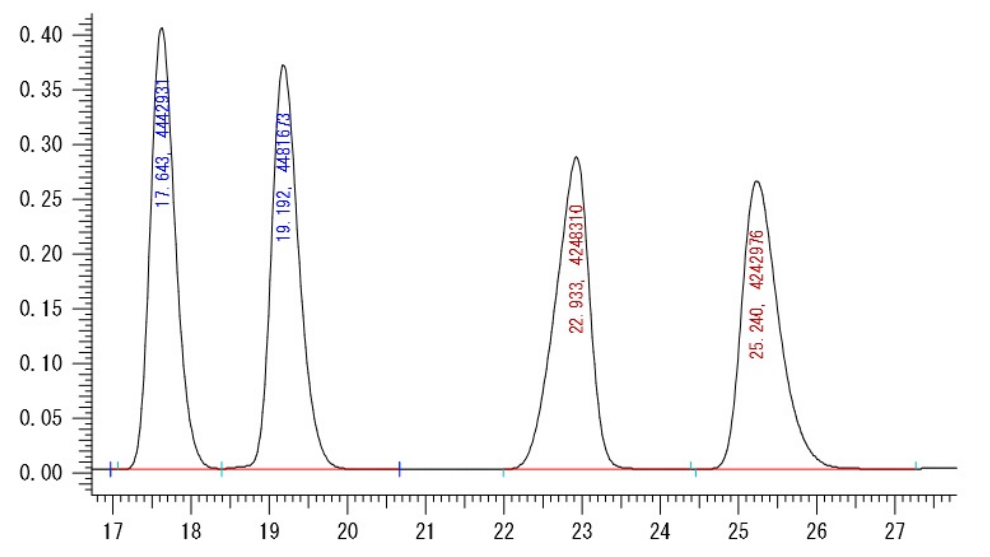

\begin{tabular}{llll}
\multicolumn{2}{l}{$r a c-3 a f$} \\
No & rt (min) & area & area (\%) \\
1 & 17.64 & 4442931 & 25.511 \\
2 & 19.19 & 4481673 & 25.733 \\
3 & 22.93 & 4248310 & 24.393 \\
4 & 25.24 & 4242976 & 24.363
\end{tabular}

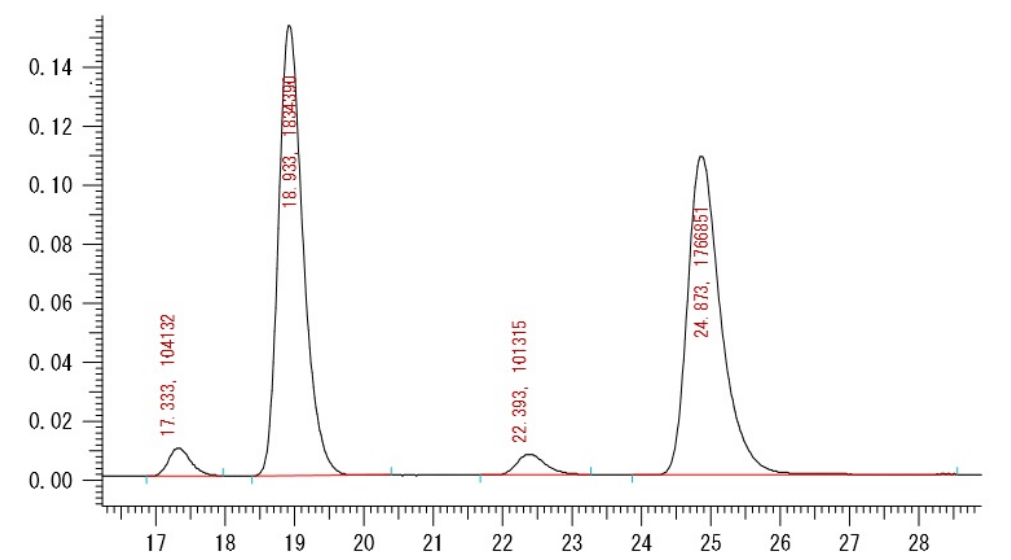

(1S)-3af

$\begin{array}{llll}\text { No } & \text { rt (min) } & \text { area } & \text { area }(\%) \\ 1 & 17.33 & 104132 & 2.736 \\ 2 & 18.93 & 1834390 & 48.189 \\ 3 & 22.39 & 101315 & 2.662 \\ 4 & 24.87 & 1766851 & 46.414\end{array}$




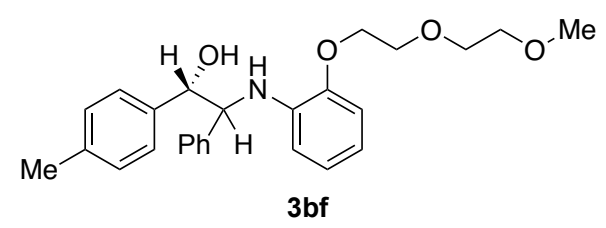

The product 3bf was purified by flash chromatography on silica gel (hexane/Et 3 N/EtOAc 5:0.5:9530:0.5:70) (Scheme 1; $72.6 \mathrm{mg}, 0.172 \mathrm{mmol}, 86 \%$ isolated yield). The diastereomeric ratio (1:1) was determined by ${ }^{1} \mathrm{H}$ NMR. Yellow oil. IR (neat) 737, 1050, 1127, 1215, 1341, 1452, 1511, 1602, 2876, $3026,3410 \mathrm{~cm}^{-1} .{ }^{1} \mathbf{H}$ NMR $\left(400 \mathrm{MHz}, \mathrm{CDCl}_{3}\right) \delta 2.30(\mathrm{~s}, 0.5 \times 3 \mathrm{H}), 2.31(\mathrm{~s}, 0.5 \times 3 \mathrm{H}), 2.85(\mathrm{brs}, 0.5$ $\times 1 \mathrm{H}), 3.14($ brs, $0.5 \times 1 \mathrm{H}), 3.37(\mathrm{~s}, 0.5 \times 3 \mathrm{H}), 3.38(\mathrm{~s}, 0.5 \times 3 \mathrm{H}), 3.57-3.62(\mathrm{~m}, 2 \mathrm{H}), 3.72-3.78(\mathrm{~m}$, $2 \mathrm{H}), 3.83-3.90(\mathrm{~m}, 2 \mathrm{H}), 4.09-4.21(\mathrm{~m}, 2 \mathrm{H}), 4.45(\mathrm{~d}, J=6.4 \mathrm{~Hz}, 0.5 \times 1 \mathrm{H}), 4.63(\mathrm{~d}, J=4.0 \mathrm{~Hz}, 0.5 \times$ $1 \mathrm{H}), 4.81(\mathrm{dd}, J=6.4,2.0 \mathrm{~Hz}, 0.5 \times 1 \mathrm{H}), 5.04(\mathrm{t}, J=5.2 \mathrm{~Hz}, 0.5 \times 1 \mathrm{H}), 5.17($ brs, $0.5 \times 1 \mathrm{H}), 5.42$ (brs, $0.5 \times 1 \mathrm{H}), 6.36(\mathrm{~m}, 1 \mathrm{H}), 6.56(\mathrm{~m}, 1 \mathrm{H}), 6.68(\mathrm{~m}, 1 \mathrm{H}), 6.76(\mathrm{~m}, 1 \mathrm{H}), 6.98-7.24(\mathrm{~m}, 9 \mathrm{H}) .{ }^{13} \mathbf{C ~ N M R}$ $\left(100.5 \mathrm{MHz}, \mathrm{CDCl}_{3}\right) \delta 21.1(\times 2 \mathrm{C}), 59.0,59.1,63.7,65.0,68.5,68.9,69.93,69.95,70.67,70.72$, 72.00, 72.02, 76.9, 78.0, 111.8, 112.0, 112.1, 112.7, 116.8, 116.9, 121.8, 122.0, 126.5, 126.7, 127.0, $127.2,127.3,127.4,127.9,128.1,128.3,128.7,137.2,137.2,137.3,137.5,137.8,138.2,139.0,140.5$, 146.2, 146.4. HRMS-DART $(\mathrm{m} / \mathrm{z})$ : $[\mathrm{M}+\mathrm{H}]^{+}$calcd for $\mathrm{C}_{26} \mathrm{H}_{32} \mathrm{~N}_{1} \mathrm{O}_{4}, 422.2326$; found, 422.2324. $[\alpha]_{\mathrm{D}}{ }^{29}+4.5\left(c 1.15, \mathrm{CHCl}_{3}\right)$. The ee value $(90 \%$ ee/90\% ee) was determined by chiral HPLC analysis [CHIRALCEL ${ }^{\circledR}$ IC-3 column, $4.6 \mathrm{~mm} \times 250 \mathrm{~mm}$, Daicel Chemical Industries, hexane/2-propanol = 95:5, $0.5 \mathrm{~mL} / \mathrm{min}, 40{ }^{\circ} \mathrm{C}, 250 \mathrm{~nm}$ UV detector, $\mathrm{t}_{\mathrm{R}}=44.5 \mathrm{~min}\left(\right.$ major $\left._{\mathrm{A}}\right), 48.1 \mathrm{~min}\left(\right.$ minor $\left._{\mathrm{B}}\right), 55.2 \mathrm{~min}$ ( minor $\left._{\mathrm{A}}\right), 100.9 \min \left(\right.$ major $\left.\left._{\mathrm{B}}\right)\right]$. The absolute configuration of $\mathbf{3 b f}$ was assigned by consideration of the stereochemical pathway.

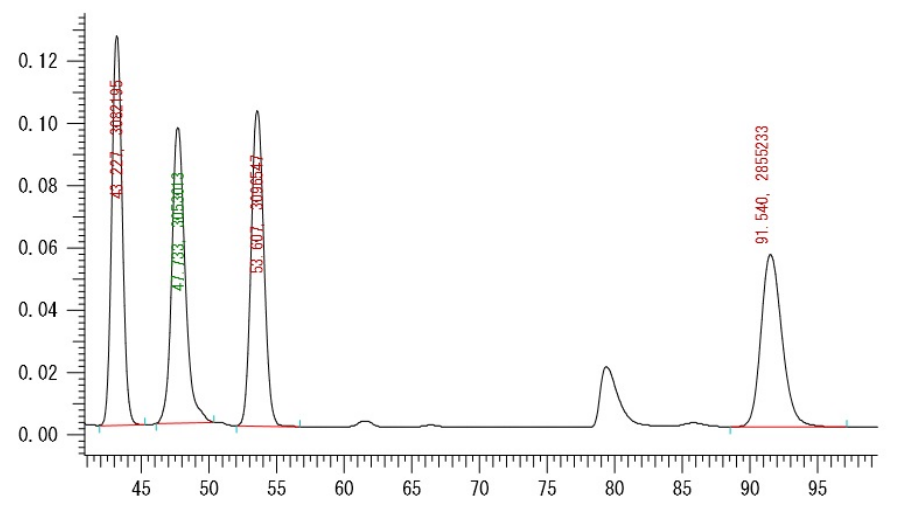

\begin{tabular}{llll}
\multicolumn{2}{l}{$r a c-3 b f$} \\
No & rt (min) & area & area (\%) \\
1 & 43.23 & 3082195 & 25.500 \\
2 & 47.73 & 3053013 & 25.259 \\
3 & 53.61 & 3096547 & 25.619 \\
4 & 91.54 & 2855233 & 23.622
\end{tabular}

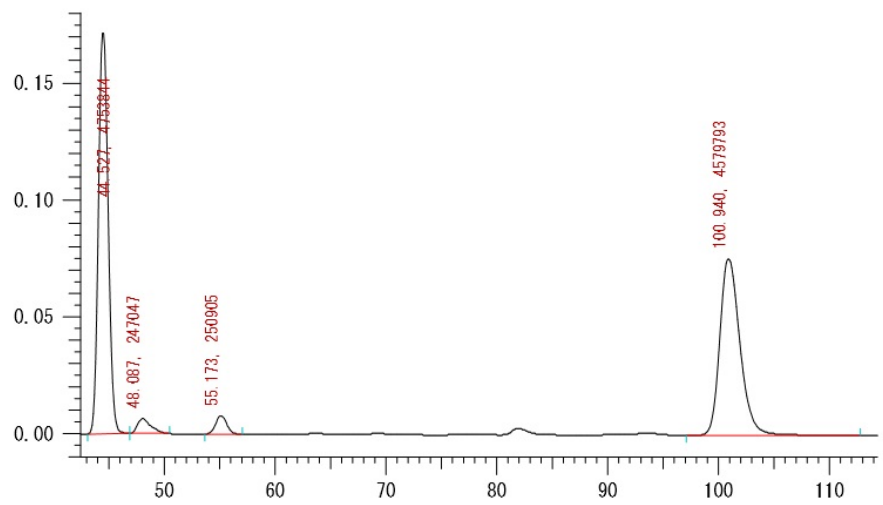

(1S)-3bf

$\begin{array}{llll}\text { No } & \text { rt (min) } & \text { area } & \text { area (\%) } \\ 1 & 44.53 & 4753844 & 48.353 \\ 2 & 48.09 & 247047 & 2.513 \\ 3 & 55.17 & 250905 & 2.552 \\ 4 & 100.94 & 4579793 & 46.582\end{array}$




\section{1-ol (3cf)}

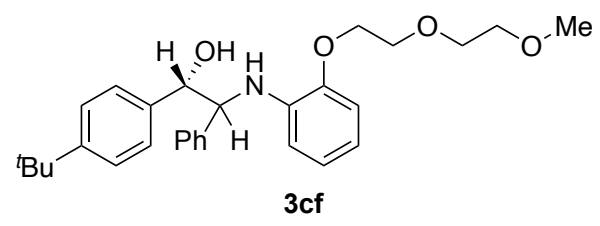

The product 3cf was purified by flash chromatography on silica gel (hexane/Et ${ }_{3} \mathrm{~N} / \mathrm{EtOAc}$ 5:0.5:95-30:0.5:70) (Scheme 1; $79.0 \mathrm{mg}, 0.170 \mathrm{mmol}, 85 \%$ isolated yield). The diastereomeric ratio (1:1) was determined by ${ }^{1} \mathrm{H}$ NMR. Yellow oil. IR (neat) 737, 1128, 1246, 1360, 1453, 1512, 1602, 2870, 2955, 3061, $3412 \mathrm{~cm}^{-1} .{ }^{1} \mathbf{H}$ NMR $\left(400 \mathrm{MHz}, \mathrm{CDCl}_{3}\right) \delta 1.28(\mathrm{~s}, 0.5 \times 9 \mathrm{H}), 1.30(\mathrm{~s}, 0.5 \times 9 \mathrm{H})$, $2.73($ brs, $0.5 \times 1 \mathrm{H}), 3.03($ brs, $0.5 \times 1 \mathrm{H}), 3.376(\mathrm{~s}, 0.5 \times 3 \mathrm{H}), 3.382(\mathrm{~s}, 0.5 \times 3 \mathrm{H}), 3.58-3.62(\mathrm{~m}, 2 \mathrm{H})$, $3.74-3.90(\mathrm{~m}, 4 \mathrm{H}), 4.06-4.21(\mathrm{~m}, 2 \mathrm{H}), 4.48(\mathrm{~d}, J=6.0 \mathrm{~Hz}, 0.5 \times 1 \mathrm{H}), 4.60(\mathrm{~d}, J=5.2 \mathrm{~Hz}, 0.5 \times 1 \mathrm{H})$, $4.84(\mathrm{~d}, J=6.0 \mathrm{~Hz}, 0.5 \times 1 \mathrm{H}), 5.02($ brs, $0.5 \times 1 \mathrm{H}), 5.17$ (brs, $0.5 \times 1 \mathrm{H}), 5.44($ brs, $0.5 \times 1 \mathrm{H}), 6.33$ $(\mathrm{m}, 1 \mathrm{H}), 6.56(\mathrm{~m}, 1 \mathrm{H}), 6.67(\mathrm{~m}, 1 \mathrm{H}), 6.76(\mathrm{~m}, 1 \mathrm{H}), 7.07(\mathrm{~m}, 1 \mathrm{H}), 7.06-7.29(\mathrm{~m}, 8 \mathrm{H}) .{ }^{13} \mathbf{C}$ NMR $(100.5$ $\left.\mathrm{MHz}_{,} \mathrm{CDCl}_{3}\right) \delta 31.3,31.3,34.4,34.5,59.0,59.1,63.8,64.7,68.5,68.9,69.9,70.0,70.7,70.7,72.0$ $(\times 2 \mathrm{C}), 78.0(\times 2 \mathrm{C}), 111.7,111.9,112.1,112.6,116.7,116.8,121.8,122.0,124.9(\times 2 \mathrm{C}), 126.3,126.4$, 127.2, 127.3, 127.3, 127.9, 128.1, 128.3, 137.4, 137.6, 137.9, 138.2, 139.1, 140.6, 146.2, 146.4, 150.6, 150.7. HRMS-DART $(\mathrm{m} / \mathrm{z})$ : $[\mathrm{M}+\mathrm{H}]^{+}$calcd for $\mathrm{C}_{29} \mathrm{H}_{38} \mathrm{~N}_{1} \mathrm{O}_{4}, 464.2795$; found, 464.2796. [ $\left.\alpha\right]_{\mathrm{D}}{ }^{29}+8.7$ (c $\left.0.29, \mathrm{CHCl}_{3}\right)$. The ee value $(90 \%$ ee/91\% ee) was determined by chiral HPLC analysis [CHIRALCEL ${ }^{\circledR}$ IC-3 column, $4.6 \mathrm{~mm} \times 250 \mathrm{~mm}$, Daicel Chemical Industries, hexane/2-propanol = 93:7, $0.5 \mathrm{~mL} / \mathrm{min}, 40{ }^{\circ} \mathrm{C}, 250 \mathrm{~nm}$ UV detector, $\mathrm{t}_{\mathrm{R}}=25.7 \mathrm{~min}\left(\right.$ minor $\left._{\mathrm{B}}\right), 28.0 \min \left(\right.$ major $\left._{\mathrm{A}}\right), 33.6 \mathrm{~min}$ $\left(\operatorname{minor}_{\mathrm{A}}\right), 75.7 \mathrm{~min}\left(\right.$ major $\left.\left._{\mathrm{B}}\right)\right]$. The absolute configuration of $\mathbf{3 c f}$ was assigned by consideration of the stereochemical pathway.

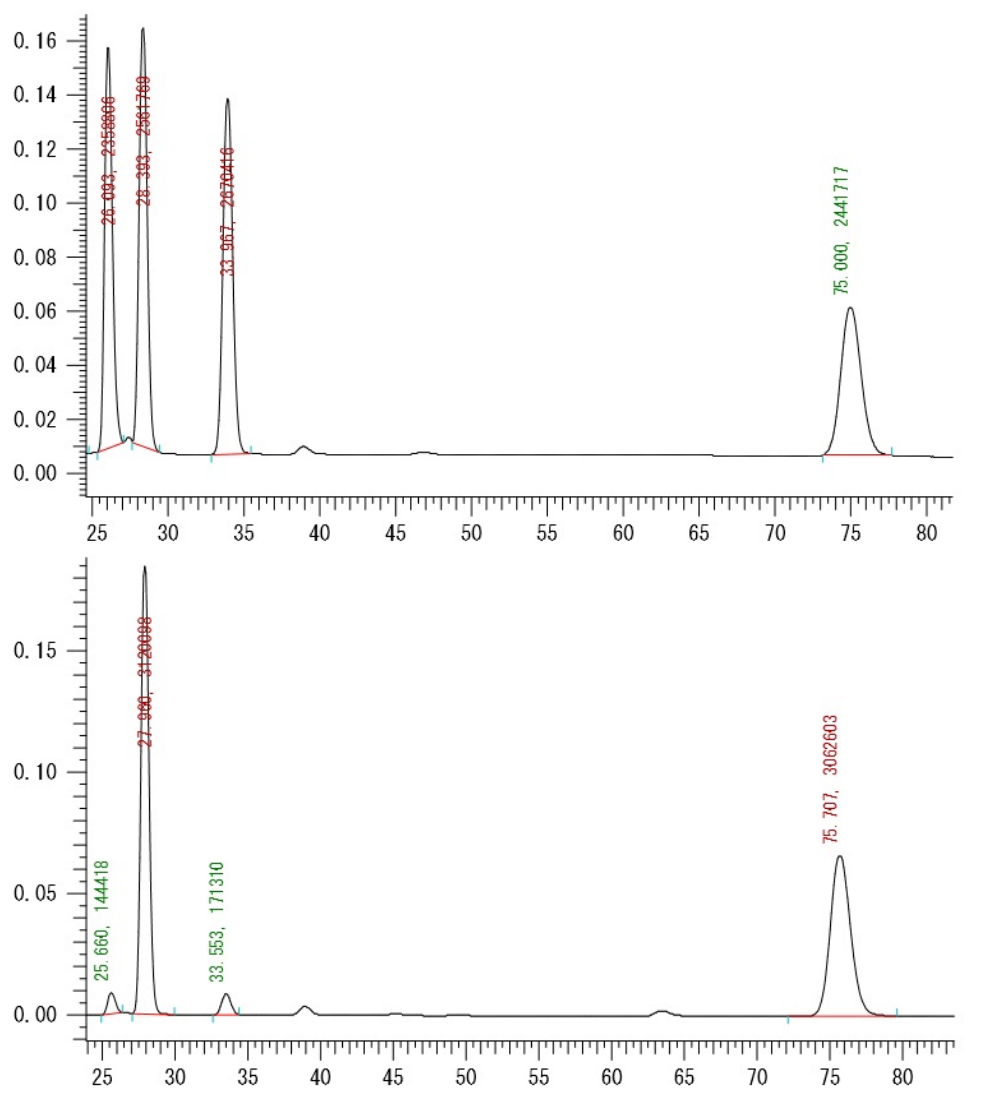

$$
r a c-3 \mathbf{c f}
$$$$
\text { No } \mathrm{rt}(\mathrm{min}) \text { area area (\%) }
$$$$
1 \quad 26.09 \quad 2358806 \quad 23.511
$$

$\begin{array}{llll}2 & 28.39 & 2561769 & 25.534\end{array}$

$\begin{array}{llll}3 & 33.97 & 2670416 & 26.617\end{array}$

$\begin{array}{llll}4 & 75.00 & 2441717 & 24.338\end{array}$

(1S)-3cf

$\begin{array}{llll}\text { No } & \text { rt (min) } & \text { area } & \text { area }(\%) \\ 1 & 25.66 & 144418 & 2.222 \\ 2 & 27.96 & 3120098 & 48.013 \\ 3 & 33.55 & 171310 & 2.636 \\ 4 & 75.71 & 3062603 & 47.128\end{array}$




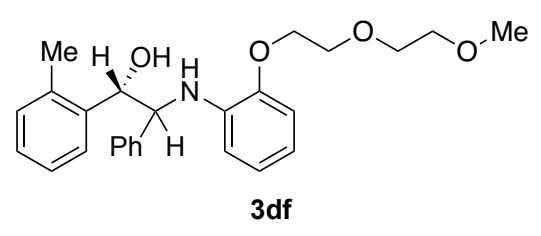

The product 3df was purified by flash chromatography on silica gel (hexane/Et $3 \mathrm{~N} / \mathrm{EtOAc}$ 5:0.5:95-30:0.5:70) (Scheme 1; $79.3 \mathrm{mg}, 0.188 \mathrm{mmol}, 94 \%$ isolated yield). The diastereomeric ratio (1:1) was determined by ${ }^{1} \mathrm{H}$ NMR. Yellow oil. IR (neat) 736, 1046, 1129, 1215, 1453, 1511, 1601, 2876, 2924, 3026, 3061, $3409 \mathrm{~cm}^{-1} .{ }^{\mathbf{1}} \mathbf{H}$ NMR (600 MHz, $\left.\mathrm{CDCl}_{3}\right) \delta 1.90(\mathrm{~s}, 0.5 \times 3 \mathrm{H}), 2.23(\mathrm{~s}, 0.5 \times$ $3 \mathrm{H}), 2.89(\mathrm{brs}, 0.5 \times 1 \mathrm{H}), 3.21(\mathrm{brs}, 0.5 \times 1 \mathrm{H}), 3.37(\mathrm{~s}, 0.5 \times 3 \mathrm{H}), 3.38(\mathrm{~s}, 0.5 \times 3 \mathrm{H}), 3.58-3.64(\mathrm{~m}$, $2 \mathrm{H}), 3.72-3.94(\mathrm{~m}, 4 \mathrm{H}), 4.11-4.24(\mathrm{~m}, 2 \mathrm{H}), 4.48(\mathrm{~d}, J=6.6 \mathrm{~Hz}, 0.5 \times 1 \mathrm{H}), 4.60(\mathrm{~d}, J=4.2 \mathrm{~Hz}, 0.5 \times$ $1 \mathrm{H}), 5.06(\mathrm{~d}, J=6.0 \mathrm{~Hz}, 0.5 \times 1 \mathrm{H}), 5.30(\mathrm{t}, J=4.8 \mathrm{~Hz}, 0.5 \times 1 \mathrm{H}), 5.36(\mathrm{brs}, 0.5 \times 1 \mathrm{H}), 5.56$ (brs, 0.5 $\times 1 \mathrm{H}), 6.30(\mathrm{~d}, J=7.8 \mathrm{~Hz}, 0.5 \times 1 \mathrm{H}), 6.36(\mathrm{~d}, J=7.8 \mathrm{~Hz}, 0.5 \times 1 \mathrm{H}), 6.57(\mathrm{~m}, 1 \mathrm{H}), 6.67(\mathrm{~m}, 1 \mathrm{H}), 6.77$ $(\mathrm{m}, 1 \mathrm{H}), 6.96(\mathrm{~d}, J=7.8 \mathrm{~Hz}, 0.5 \times 1 \mathrm{H}), 7.04-7.23(\mathrm{~m}, 8 \mathrm{H}), 7.64(\mathrm{~d}, J=7.8 \mathrm{~Hz}, 0.5 \times 1 \mathrm{H}) .{ }^{13} \mathbf{C} \mathbf{N M R}$ $\left(150.9 \mathrm{MHz}, \mathrm{CDCl}_{3}\right) \delta 18.9,19.1,59.0,59.0,62.5,64.4,68.5,68.9,69.9,70.0,70.6,70.7,72.0,73.3$, $73.3,74.0,111.8,112.1,112.2,112.7,116.8,116.9,121.9,122.0,125.7,126.0,126.0,126.6,126.6$, $127.2,127.3,127.4,127.5,127.9,128.1,128.2,130.0,130.10,134.9,135.6,137.6,138.3,138.7$, 138.8, 139.3, 140.2, 146.2, 146.4. HRMS-DART $(\mathrm{m} / \mathrm{z}):[\mathrm{M}+\mathrm{H}]^{+}$calcd for $\mathrm{C}_{26} \mathrm{H}_{32} \mathrm{~N}_{1} \mathrm{O}_{4}, 422.2326$; found, 422.2328. $[\alpha]_{\mathrm{D}}{ }^{29}+26.8\left(c 1.13, \mathrm{CHCl}_{3}\right)$. The ee value $(79 \%$ ee/79\% ee $)$ was determined by chiral HPLC analysis [CHIRALCEL ${ }^{\circledR}$ IC-3 column, $4.6 \mathrm{~mm} \times 250 \mathrm{~mm}$, Daicel Chemical Industries, hexane/2-propanol $=90: 10,0.5 \mathrm{~mL} / \mathrm{min}, 40{ }^{\circ} \mathrm{C}, 250 \mathrm{~nm} \mathrm{UV}$ detector, $\mathrm{t}_{\mathrm{R}}=17.8 \mathrm{~min}$ ( major $\left._{\mathrm{A}}\right), 20.3$ $\min \left(\operatorname{minor}_{\mathrm{B}}\right), 21.4 \min \left(\operatorname{minor}_{\mathrm{A}}\right), 27.4 \min \left(\right.$ major $\left.\left._{\mathrm{B}}\right)\right]$. The absolute configuration of $\mathbf{3 d f}$ was assigned by consideration of the stereochemical pathway.

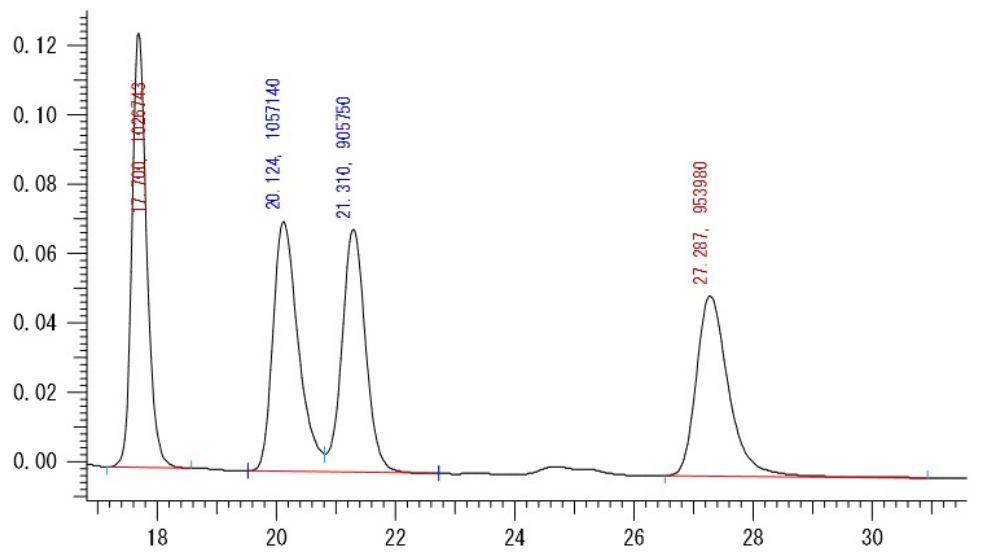

$r a c-3 d f$

$\begin{array}{llll}\text { No } & \text { rt }(\min ) & \text { area } & \text { area }(\%) \\ 1 & 17.70 & 1026743 & 26.036 \\ 2 & 20.12 & 1057140 & 26.806 \\ 3 & 21.31 & 905750 & 22.968 \\ 4 & 27.29 & 953980 & 24.191\end{array}$

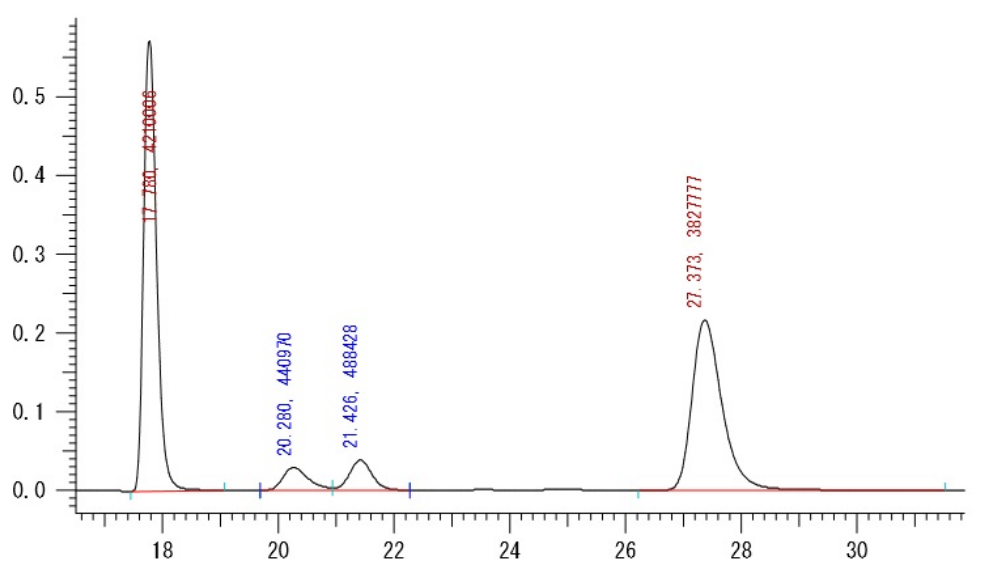

(1S)-3df

$\begin{array}{llll}\text { No } & \text { rt (min) } & \text { area } & \text { area (\%) } \\ 1 & 17.78 & 4210006 & 46.949 \\ 2 & 20.28 & 440970 & 4.918 \\ 3 & 21.43 & 488428 & 5.447 \\ 4 & 27.37 & 3827777 & 42.687\end{array}$




\section{1-ol (3ef)}

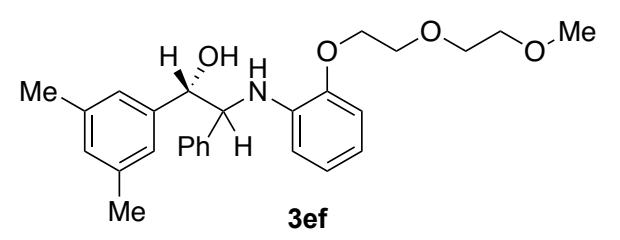

The product 3ef was purified by flash chromatography on silica gel (hexane/Et 3 N/EtOAc 5:0.5:9530:0.5:70) (Scheme 1; $86.2 \mathrm{mg}, 0.198 \mathrm{mmol}, 99 \%$ isolated yield). The diastereomeric ratio (1:1) was determined by ${ }^{1} \mathrm{H}$ NMR. Yellow oil. IR (neat) 736, 1053, 1127, 1215, 1542, 1511, 1601, 2876, 2917 , 3027, 3060, $3413 \mathrm{~cm}^{-1} .{ }^{1} \mathbf{H}$ NMR $\left(600 \mathrm{MHz} \mathrm{CDCl}_{3}\right) \delta 2.23(\mathrm{~s}, 0.5 \times 6 \mathrm{H}), 2.24(\mathrm{~s}, 0.5 \times 6 \mathrm{H}), 2.80$ (brs, $0.5 \times 1 \mathrm{H}), 3.12($ brs, $0.5 \times 1 \mathrm{H}), 3.369(\mathrm{~s}, 0.5 \times 3 \mathrm{H}), 3.373(\mathrm{~s}, 0.5 \times 3 \mathrm{H}), 3.56-3.61(\mathrm{~m}, 2 \mathrm{H})$, $3.70-3.90(\mathrm{~m}, 4 \mathrm{H}), 4.09-4.19(\mathrm{~m}, 2 \mathrm{H}), 4.44(\mathrm{~d}, J=6.0 \mathrm{~Hz}, 0.5 \times 1 \mathrm{H}), 4.60(\mathrm{~d}, J=4.8 \mathrm{~Hz}, 0.5 \times 1 \mathrm{H})$, $4.77(\mathrm{~d}, J=6.0 \mathrm{~Hz}, 0.5 \times 1 \mathrm{H}), 4.96(\mathrm{t}, J=4.4 \mathrm{~Hz}, 0.5 \times 1 \mathrm{H}), 5.15$ (brs, $0.5 \times 1 \mathrm{H}), 5.41$ (brs, $0.5 \times$ $1 \mathrm{H}), 6.35(\mathrm{~d}, J=7.8 \mathrm{~Hz}, 1 \mathrm{H}), 6.56(\mathrm{~m}, 1 \mathrm{H}), 6.66-6.87(\mathrm{~m}, 5 \mathrm{H}), 7.13-7.24(\mathrm{~m}, 5 \mathrm{H}) .{ }^{13} \mathbf{C}$ NMR $(150.9$ $\left.\mathrm{MHz} \mathrm{CDCl}_{3}\right) \delta 21.2(\times 2 \mathrm{C}), 59.0,59.0,63.7,63.7,64.9,68.4,68.8,69.8,69.9,70.7,72.0,72.0,77.1$, 78.1, 111.8, 112.0, 112.1, 112.6, 116.7, 116.8, 121.8, 122.0, 124.4, 124.5, 127.2, 127.3, 127.9, 128.0, $128.2(\times 2 \mathrm{C}), 129.2,129.3,137.3,137.4,137.5,138.2,139.0,140.2,140.5,140.7,146.2,146.3$. HRMS-DART $(\mathrm{m} / z)$ : $[\mathrm{M}+\mathrm{H}]^{+}$calcd for $\mathrm{C}_{27} \mathrm{H}_{34} \mathrm{~N}_{1} \mathrm{O}_{4}, 436.2482$; found, 436.2482. [ $\left.\alpha\right]_{\mathrm{D}}{ }^{29}+3.7(\mathrm{c} 1.06$, $\left.\mathrm{CHCl}_{3}\right)$. The ee value $\left(85 \%\right.$ ee/ $84 \%$ ee) was determined by chiral HPLC analysis [CHIRALCEL ${ }^{\circledR}$ IC$3+$ IC column, $4.6 \mathrm{~mm} \times 250 \mathrm{~mm}$, Daicel Chemical Industries, hexane/2-propanol = 95:5, 0.5 $\mathrm{mL} / \mathrm{min}, 40{ }^{\circ} \mathrm{C}, 250 \mathrm{~nm} \mathrm{UV}$ detector, $\mathrm{t}_{\mathrm{R}}=62.1 \min \left(\operatorname{minor}_{\mathrm{B}}\right), 65.9 \min \left(\right.$ major $\left._{\mathrm{B}}\right), 77.9 \min \left(\operatorname{minor}_{\mathrm{A}}\right)$, $131.0 \mathrm{~min}\left(\right.$ major $\left.\left._{\mathrm{A}}\right)\right]$. The absolute configuration of $3 \mathbf{e f}$ was assigned by consideration of the stereochemical pathway.

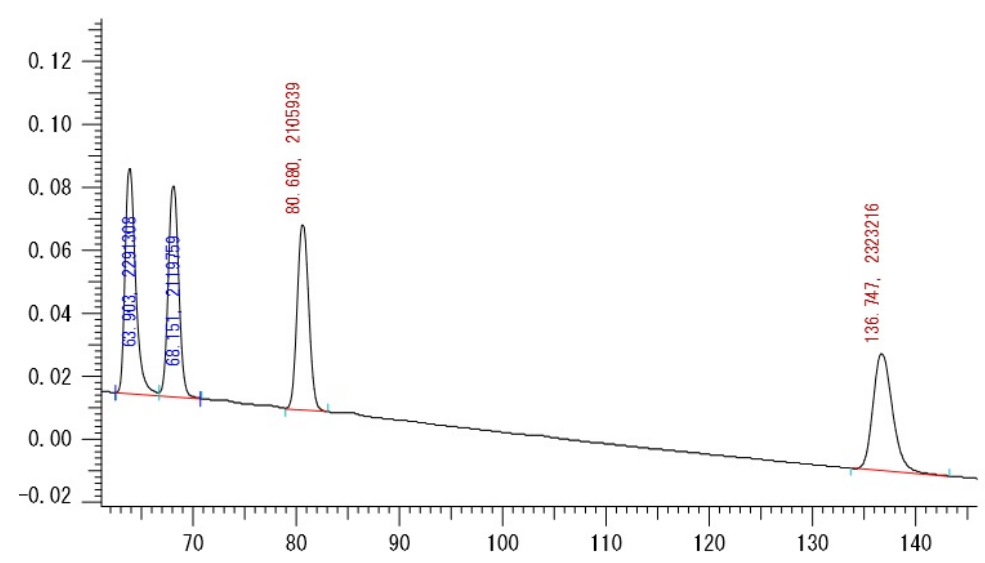

\section{rac-3ef}

No $\mathrm{rt}(\mathrm{min})$ area $\quad$ area $(\%)$

$1 \quad 63.90 \quad 2291308 \quad 25.919$

$\begin{array}{llll}2 & 68.15 & 2119759 & 23.979\end{array}$

$\begin{array}{llll}3 & 80.68 & 2105939 & 23.822\end{array}$

$4 \quad 136.75 \quad 2323216 \quad 26.280$

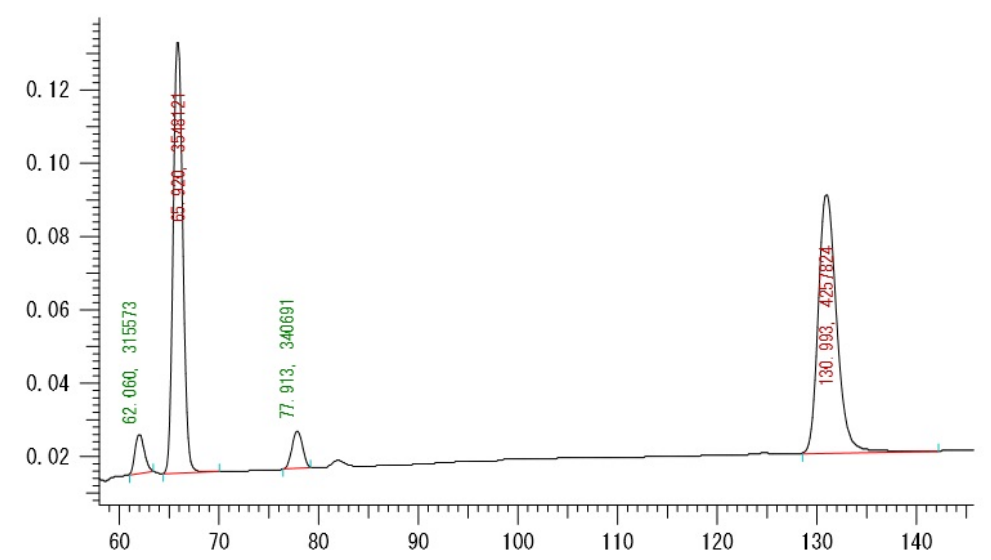

(1S)-3ef

$\begin{array}{llll}\text { No } & \text { rt }(\mathrm{min}) & \text { area } & \text { area }(\%) \\ 1 & 62.06 & 315573 & 3.729 \\ 2 & 65.92 & 3548121 & 41.929 \\ 3 & 77.91 & 340691 & 4.026 \\ 4 & 130.99 & 4257824 & 50.316\end{array}$



ol (3ff)

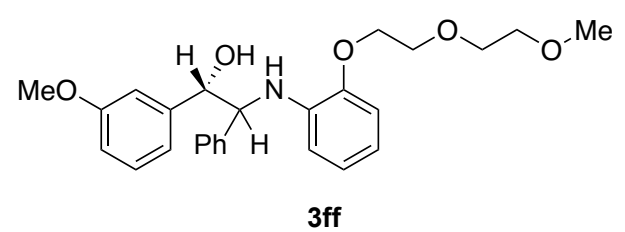

The product $3 f f$ was purified by flash chromatography on silica gel (hexane/Et ${ }_{3} \mathrm{~N} / \mathrm{EtOAc}$ 5:0.5:9530:0.5:70) (Scheme 1; $81.7 \mathrm{mg}, 0.187 \mathrm{mmol}, 93 \%$ isolated yield). The diastereomeric ratio (1:1) is determined by ${ }^{1} \mathrm{H}$ NMR. Yellow oil. IR (neat) 704, 1045, 1106, 1257, 1453, 1511, 1601, 2834, 2879 , 2930, 3027, 3062, $3408 \mathrm{~cm}^{-1} .{ }^{1} \mathbf{H}$ NMR $\left(400 \mathrm{MHz}, \mathrm{CDCl}_{3}\right) \delta 3.03(\mathrm{~m}, 0.5 \times 1 \mathrm{H}), 3.28(\mathrm{brs}, 0.5 \times 1 \mathrm{H})$, $3.366(\mathrm{~s}, 0.5 \times 3 \mathrm{H}), 3.375(\mathrm{~s}, 0.5 \times 3 \mathrm{H}), 3.57-3.62(\mathrm{~m}, 2 \mathrm{H}), 3.64(\mathrm{~s}, 0.5 \times 3 \mathrm{H}), 3.67(\mathrm{~s}, 0.5 \times 3 \mathrm{H})$, $3.72-3.78(\mathrm{~m}, 2 \mathrm{H}), 3.83-3.90(\mathrm{~m}, 2 \mathrm{H}), 4.10-4.21(\mathrm{~m}, 2 \mathrm{H}), 4.45(\mathrm{~d}, J=6.4 \mathrm{~Hz}, 0.5 \times 1 \mathrm{H}), 4.65$ (brs, $0.5 \times 1 \mathrm{H}), 4.81(\mathrm{~m}, 0.5 \times 1 \mathrm{H}), 5.07(\mathrm{t}, J=5.2 \mathrm{~Hz}, 0.5 \times 1 \mathrm{H}), 5.24$ (brs, $0.5 \times 1 \mathrm{H}), 5.42$ (brs, $0.5 \times$ $1 \mathrm{H}), 6.37(\mathrm{~m}, 1 \mathrm{H}), 6.55-6.79(\mathrm{~m}, 6 \mathrm{H}), 7.11-7.22(\mathrm{~m}, 6 \mathrm{H}) .{ }^{13} \mathbf{C} \mathbf{N M R}\left(100.5 \mathrm{MHz}, \mathrm{CDCl}_{3}\right) \delta 55.0$, 55.1, 59.0, 59.0, 63.6, 65.1, 68.6, 69.0, 69.9, 69.9, 70.6, 70.7, $72.0(\times 2 \mathrm{C}), 76.8,78.1,111.7,111.8$, $112.1,112.2,112.8(\times 2 \mathrm{C}), 113.4,113.7,116.9,117.0,118.8,119.1,121.9,122.1,127.3,127.3,127.4$, 127.9, 128.0, 128.3, 128.9, 129.0, 137.5, 138.2, 138.8, 140.3, 142.0, 142.4, 146.2, 146.4, 159.3, 159.3. HRMS-DART $(\mathrm{m} / z):[\mathrm{M}+\mathrm{H}]^{+}$calcd for $\mathrm{C}_{26} \mathrm{H}_{32} \mathrm{~N}_{1} \mathrm{O}_{5}, 438.2275$; found, 438.2276. [ $\left.\alpha\right]_{\mathrm{D}}{ }^{29}-1.0(c 0.97$, $\left.\mathrm{CHCl}_{3}\right)$. The ee value $\left(71 \%\right.$ ee/70\% ee) was determined by chiral HPLC analysis [CHIRALCEL ${ }^{\circledR}$ IC3 column, $4.6 \mathrm{~mm} \times 250 \mathrm{~mm}$, Daicel Chemical Industries, hexane $/ 2$-propanol $=95: 5,1.0 \mathrm{~mL} / \mathrm{min}$, $40{ }^{\circ} \mathrm{C}, 250 \mathrm{~nm} \mathrm{UV}$ detector, $\mathrm{t}_{\mathrm{R}} 34.0 \mathrm{~min}\left(\operatorname{minor}_{\mathrm{A}}\right), 39.3 \mathrm{~min}$ (major $\left.{ }_{\mathrm{B}}\right), 41.5 \mathrm{~min}$ (minor $\left.\mathrm{B}\right), 95.5 \mathrm{~min}$ $\left(\right.$ major $\left.\left._{\mathrm{A}}\right)\right]$. The absolute configuration of $\mathbf{3 f f}$ was assigned by consideration of the stereochemical pathway.

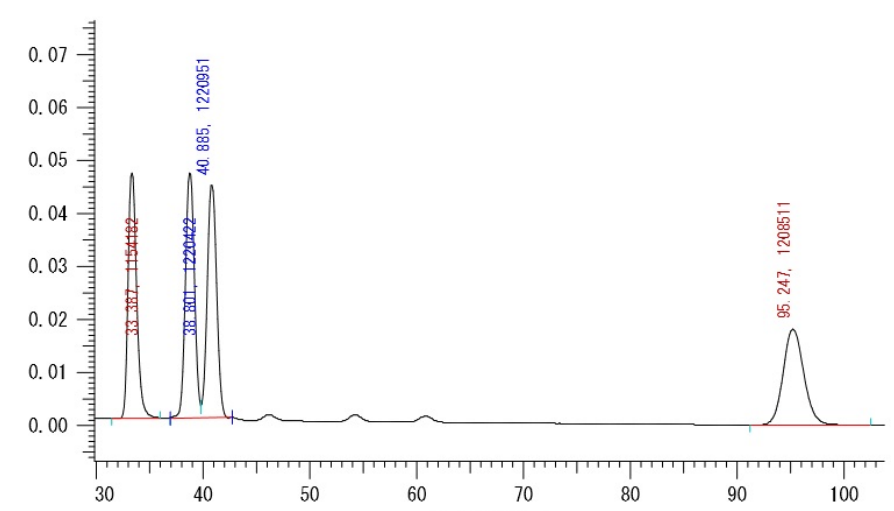

\section{$r a c-3 f f$}

$\begin{array}{llll}\text { No } & \text { rt (min) } & \text { area } & \text { area }(\%) \\ 1 & 33.39 & 1154182 & 24.025 \\ 2 & 38.80 & 1220422 & 25.404 \\ 3 & 40.89 & 1220951 & 25.415 \\ 4 & 95.25 & 1208511 & 25.156\end{array}$

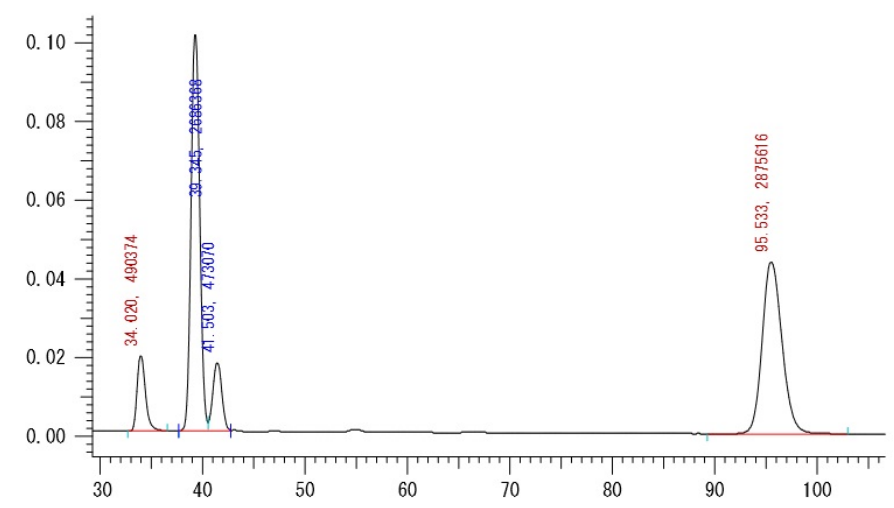

(1S)-3ff

$\begin{array}{llll}\text { No } & \text { rt (min) } & \text { area } & \text { area }(\%) \\ 1 & 34.02 & 490374 & 7.515 \\ 2 & 39.35 & 2686368 & 41.168 \\ 3 & 41.50 & 473070 & 7.250 \\ 4 & 95.53 & 2875616 & 44.068\end{array}$




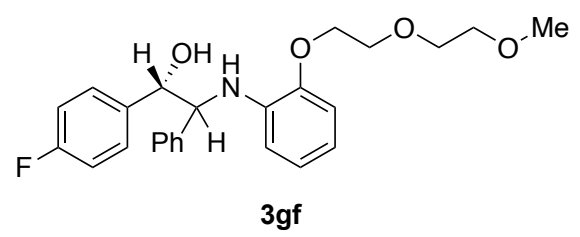

The product 3gf was purified by flash chromatography on silica gel (hexane/Et 3 N/EtOAc 5:0.5:9530:0.5:70) (Scheme 1; $80.5 \mathrm{mg}, 0.189 \mathrm{mmol}, 95 \%$ isolated yield). The diastereomeric ratio (1:1) was determined by ${ }^{1} \mathrm{H}$ NMR. Yellow oil. IR (neat) 738, 1054, 1128, 1219, 1452, 1509, 1602, 2877, 2926, $3062,3419 \mathrm{~cm}^{-1} .{ }^{1} \mathbf{H}$ NMR $\left(400 \mathrm{MHz}, \mathrm{CDCl}_{3}\right) \delta 3.15$ (brs, $\left.0.5 \times 1 \mathrm{H}\right), 3.365(\mathrm{~s}, 0.5 \times 3 \mathrm{H}), 3.373(\mathrm{~s}$, $0.5 \times 3 \mathrm{H}), 3.47($ brs, $0.5 \times 1 \mathrm{H}), 3.58-3.63(\mathrm{~m}, 2 \mathrm{H}), 3.73-3.79(\mathrm{~m}, 2 \mathrm{H}), 3.84-3.91(\mathrm{~m}, 2 \mathrm{H}), 4.14-4.19$ $(\mathrm{m}, 2 \mathrm{H}), 4.40(\mathrm{~d}, J=7.2 \mathrm{~Hz}, 0.5 \times 1 \mathrm{H}), 4.62(\mathrm{~d}, J=4.4 \mathrm{~Hz}, 0.5 \times 1 \mathrm{H}), 4.81(\mathrm{~d}, J=6.8 \mathrm{~Hz}, 0.5 \times 1 \mathrm{H})$, $5.09(\mathrm{t}, J=4.8 \mathrm{~Hz}, 0.5 \times 1 \mathrm{H}), 5.25$ (brs, $0.5 \times 1 \mathrm{H}), 5.41$ (brs, $0.5 \times 1 \mathrm{H}), 6.39(\mathrm{~m}, 1 \mathrm{H}), 6.59(\mathrm{~m}, 1 \mathrm{H})$, $6.70(\mathrm{~m}, 1 \mathrm{H}), 6.78(\mathrm{~m}, 1 \mathrm{H}), 6.89-6.96(\mathrm{~m}, 2 \mathrm{H}), 7.04-7.26(\mathrm{~m}, 7 \mathrm{H}) .{ }^{13} \mathbf{C}$ NMR $\left(100.5 \mathrm{MHz}, \mathrm{CDCl}_{3}\right)$ $\delta 59.0,59.0,63.8,65.4,68.7,69.1,70.0(\times 2 \mathrm{C}), 70.6,70.7,72.0(\times 2 \mathrm{C}), 76.2,77.6,111.9,112.2$, 112.4, 113.1, $114.8(\mathrm{~d}, J=21.1 \mathrm{~Hz}), 114.8(\mathrm{~d}, J=20.6 \mathrm{~Hz}), 117.1,117.2,122.0,122.2,127.3,127.4$, $127.8(\times 2 \mathrm{C}), 128.1,128.2(\mathrm{~d}, J=8.1 \mathrm{~Hz}), 128.3,128.4(\mathrm{~d}, J=8.1 \mathrm{~Hz}), 136.2(\mathrm{~d}, J=2.9 \mathrm{~Hz}), 136.6$ $(\mathrm{d}, J=3.4 \mathrm{~Hz}), 137.5,138.2,138.5,140.1,146.3,146.4,162.2(\mathrm{~d}, J=245.3 \mathrm{~Hz}, \times 2 \mathrm{C})$. HRMSDART $(m / z)$ : $[\mathrm{M}+\mathrm{H}]^{+}$calcd for $\mathrm{C}_{25} \mathrm{H}_{29} \mathrm{~F}_{1} \mathrm{~N}_{1} \mathrm{O}_{4}, 426.2075$; found, 426.2079. [ $\left.\alpha\right]_{\mathrm{D}}{ }^{29}+10.0(c 0.64$, $\left.\mathrm{CHCl}_{3}\right)$. The ee value $\left(90 \%\right.$ ee/91\% ee) was determined by chiral HPLC analysis [CHIRALCEL ${ }^{\circledR}$ AD-3 column, $4.6 \mathrm{~mm} \times 250 \mathrm{~mm}$, Daicel Chemical Industries, hexane/2-propanol =97:3, $0.5 \mathrm{~mL} / \mathrm{min}$, $40{ }^{\circ} \mathrm{C}, 250 \mathrm{~nm} \mathrm{UV}$ detector, $\mathrm{t}_{\mathrm{R}}=94.6 \min \left(\right.$ minor $\left._{\mathrm{A}}\right), 107.1 \min \left(\right.$ major $\left._{\mathrm{A}}\right), 113.1 \min \left(\right.$ minor $\left._{\mathrm{B}}\right), 165.0$ $\min \left(\right.$ major $\left.\left._{\mathrm{B}}\right)\right]$. The absolute configuration of $\mathbf{3 g f} \mathbf{f}$ was assigned by consideration of the stereochemical pathway.

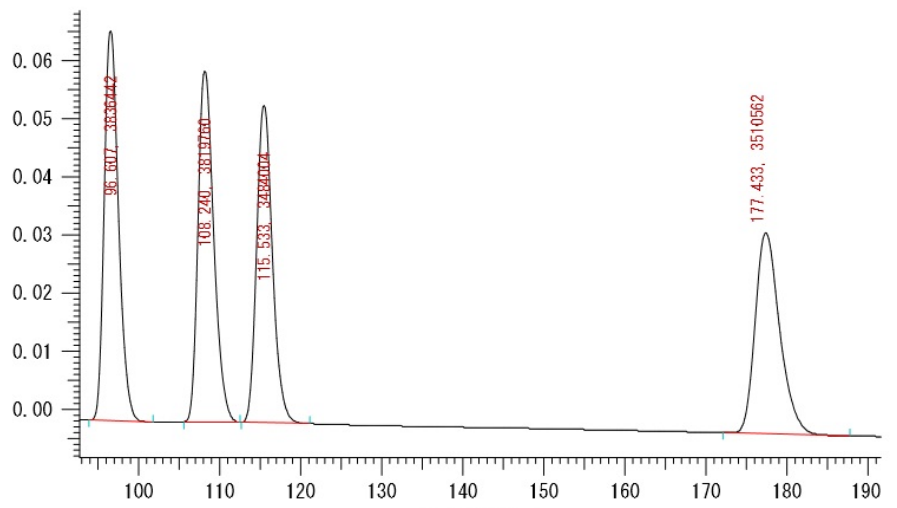

\begin{tabular}{llll}
\multicolumn{2}{l}{$r a c-3 g f$} \\
No & rt (min) & area & area (\%) \\
1 & 96.61 & 3836442 & 26.186 \\
2 & 108.24 & 3819760 & 26.072 \\
3 & 115.53 & 3484004 & 23.780 \\
4 & 177.43 & 3510562 & 23.962
\end{tabular}

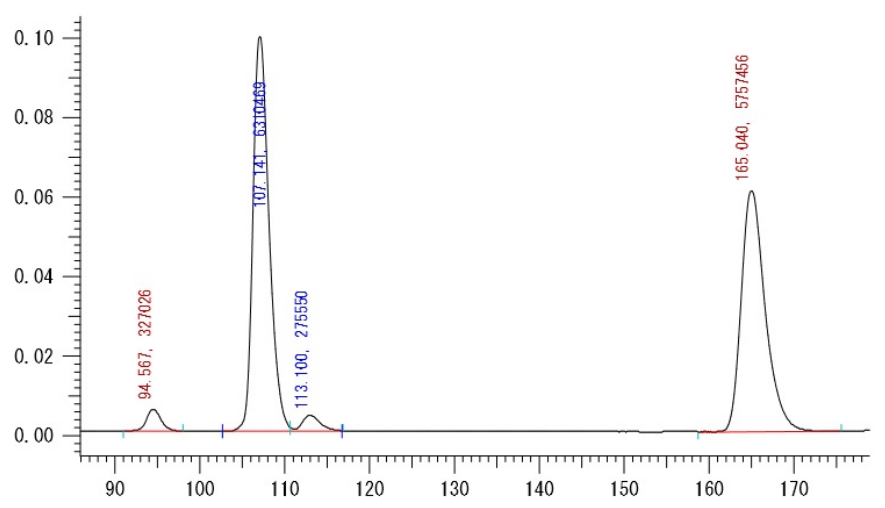

(1S)-3gf

$\begin{array}{llll}\text { No } & \mathrm{rt}(\mathrm{min}) & \text { area } & \text { area }(\%) \\ 1 & 94.57 & 327026 & 2.581 \\ 2 & 107.14 & 6310469 & 49.804 \\ 3 & 113.10 & 275550 & 2.175 \\ 4 & 165.04 & 5757456 & 45.440\end{array}$



(3hf)

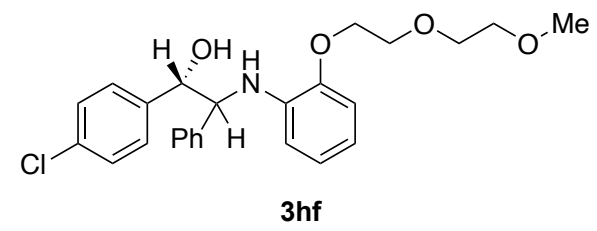

The product 3 hf was purified by flash chromatography on silica gel (hexane/Et $3 \mathrm{~N} / \mathrm{EtOAc}$ 5:0.5:95-30:0.5:70) (Scheme 1; 62.0 mg, 0.151 mmol, 70\% isolated yield). The diastereomeric ratio (1:1) was determined by ${ }^{1} \mathrm{H}$ NMR. Yellow oil. IR (neat) 738, 1055, 1092, 1215, 1452, 1511, 1602, 2878, 2925, 3028, 3062, $3413 \mathrm{~cm}^{-1} .{ }^{1} \mathbf{H}$ NMR (400 MHz, $\left.\mathrm{CDCl}_{3}\right) \delta 3.25$ (brs, $\left.0.5 \times 1 \mathrm{H}\right), 3.36(\mathrm{~s}, 0.5$ $\times 3 \mathrm{H}), 3.37(\mathrm{~s}, 0.5 \times 3 \mathrm{H}), 3.50(\mathrm{brs}, 0.5 \times 1 \mathrm{H}), 3.58-3.63(\mathrm{~m}, 2 \mathrm{H}), 3.70-3.78(\mathrm{~m}, 2 \mathrm{H}), 3.84-3.91(\mathrm{~m}$, $2 \mathrm{H}), 4.12-4.21(\mathrm{~m}, 2 \mathrm{H}), 4.40(\mathrm{~d}, J=6.8 \mathrm{~Hz}, 0.5 \times 1 \mathrm{H}), 4.63(\mathrm{~d}, J=4.0 \mathrm{~Hz}, 0.5 \times 1 \mathrm{H}), 4.81(\mathrm{~d}, J=$ $6.8 \mathrm{~Hz}, 0.5 \times 1 \mathrm{H}), 5.09(\mathrm{t}, J=4.4 \mathrm{~Hz}, 0.5 \times 1 \mathrm{H}), 5.23(\mathrm{brs}, 0.5 \times 1 \mathrm{H}), 5.40($ brs, $0.5 \times 1 \mathrm{H}), 6.39(\mathrm{~m}$, $1 \mathrm{H}), 6.59(\mathrm{~m}, 1 \mathrm{H}), 6.71(\mathrm{~m}, 1 \mathrm{H}), 6.78(\mathrm{~m}, 1 \mathrm{H}), 7.01(\mathrm{~m}, 1 \mathrm{H}), 7.07-7.15(\mathrm{~m}, 3 \mathrm{H}), 7.18-7.22(\mathrm{~m}, 5 \mathrm{H})$. ${ }^{13} \mathbf{C}$ NMR $\left(100.5 \mathrm{MHz}, \mathrm{CDCl}_{3}\right) \delta 59.0,59.0,63.6,65.2,68.6,69.0,69.9(\times 2 \mathrm{C}), 70.6,70.6,72.0(\times$ 2C), 76.1, 77.5, 111.9, 112.1, 112.4, 113.0, 117.1, 117.2, 122.0, 122.1, 127.4, 127.4, 127.8, 127.9, $128.0,128.1(\times 2 \mathrm{C}), 128.2,128.4(\times 2 \mathrm{C}), 133.2,133.2,137.4,138.1,138.3,139.0,139.4,140.0$, 146.3, 146.4. HRMS-DART (m/z): $[\mathrm{M}+\mathrm{H}]^{+}$calcd for $\mathrm{C}_{25} \mathrm{H}_{29} \mathrm{Cl}_{1} \mathrm{~N}_{1} \mathrm{O}_{4}, 442.1780$; found, 442.1768. $[\alpha]_{\mathrm{D}}{ }^{29}+2.0\left(c 1.23, \mathrm{CHCl}_{3}\right)$. The ee value $(87 \%$ ee/ $88 \%$ ee $)$ was determined by chiral HPLC analysis [CHIRALCEL ${ }^{\circledR}$ AD-3 + AD-H column, $4.6 \mathrm{~mm} \times 250 \mathrm{~mm}$, Daicel Chemical Industries, hexane/2propanol $=96: 4,1.0 \mathrm{~mL} / \mathrm{min}, 40{ }^{\circ} \mathrm{C}, 250 \mathrm{~nm} \mathrm{UV}$ detector, $\mathrm{t}_{\mathrm{R}}=87.6 \mathrm{~min}\left(\right.$ minor $\left._{\mathrm{A}}\right), 94.2 \mathrm{~min}\left(\right.$ major $\left._{\mathrm{A}}\right)$, $97.6 \min \left(\right.$ minor $\left._{\mathrm{B}}\right), 160.7 \min \left(\right.$ major $\left.\left._{\mathrm{B}}\right)\right]$. The absolute configuration of $\mathbf{3 h f}$ was assigned by consideration of the stereochemical pathway.

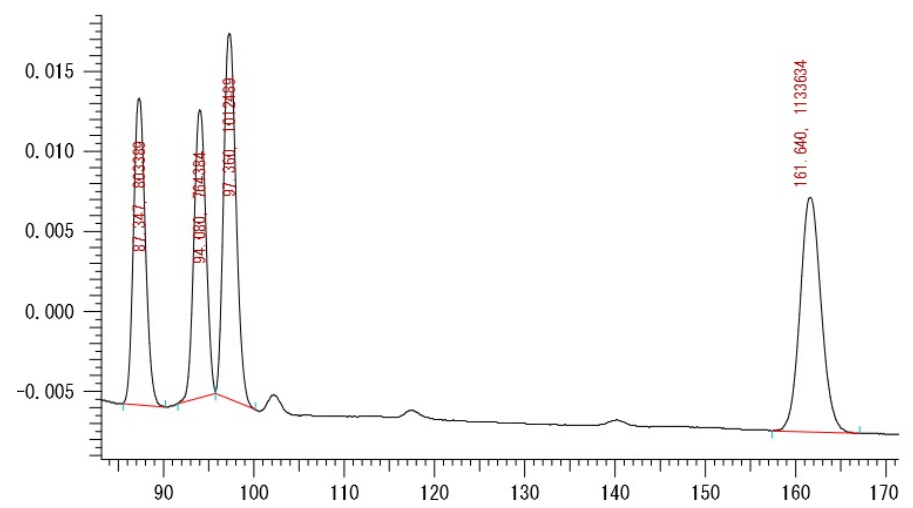

\begin{tabular}{llll}
\multicolumn{2}{l}{$r a c-3 h f$} \\
No & rt (min) & area & area (\%) \\
1 & 87.35 & 803389 & 21.632 \\
2 & 94.08 & 764384 & 20.582 \\
3 & 97.36 & 1012489 & 27.262 \\
4 & 161.64 & 1133634 & 30.524
\end{tabular}

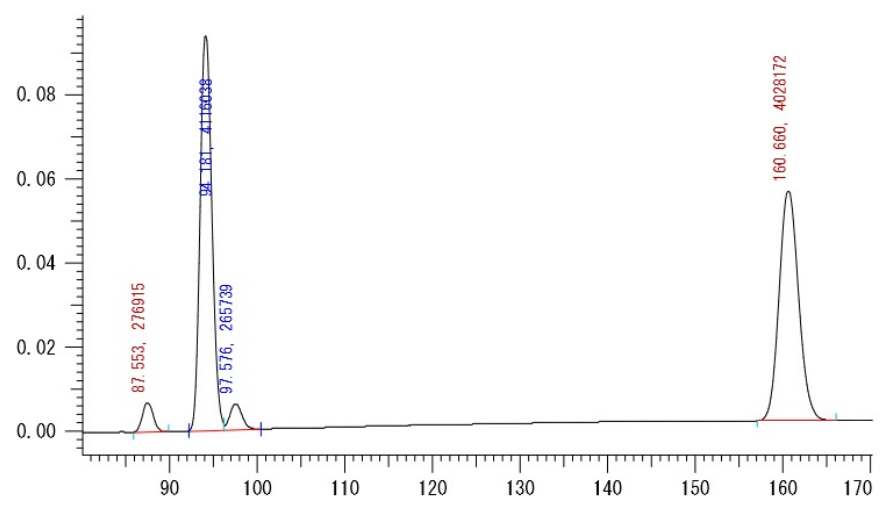

(1S)-3hf

$\begin{array}{llll}\text { No } & \text { rt (min) } & \text { area } & \text { area }(\%) \\ 1 & 87.55 & 276915 & 3.188 \\ 2 & 94.18 & 4116038 & 47.382 \\ 3 & 97.58 & 265739 & 3.059 \\ 4 & 160.66 & 4028172 & 46.371\end{array}$




\section{1-ol (3if)}

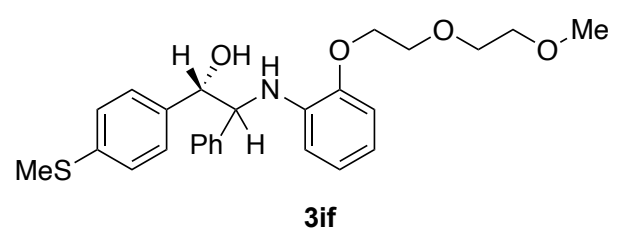

The product 3if was purified by flash chromatography on silica gel (hexane/Et 3 N/EtOAc 5:0.5:9530:0.5:70) (Scheme 1; $58.2 \mathrm{mg}, 0.128 \mathrm{mmol}, 64 \%$ isolated yield). The diastereomeric ratio (1:1) was determined by ${ }^{1} \mathrm{H}$ NMR. Yellow oil. IR (neat) 737, 1094, 1215, 1441, 1512, 1601, 2877, 2920, 3026, $3414 \mathrm{~cm}^{-1} .{ }^{1} \mathbf{H}$ NMR $\left(400 \mathrm{MHz}, \mathrm{CDCl}_{3}\right) \delta 2.44(\mathrm{~s}, 0.5 \times 3 \mathrm{H}), 2.45(\mathrm{~s}, 0.5 \times 3 \mathrm{H}), 3.02(\mathrm{~d}, J=5.6 \mathrm{~Hz}$, $0.5 \times 1 \mathrm{H}), 3.31(\mathrm{brs}, 0.5 \times 1 \mathrm{H}), 3.369(\mathrm{~s}, 0.5 \times 3 \mathrm{H}), 3.375(\mathrm{~s}, 0.5 \times 3 \mathrm{H}), 3.58-3.64(\mathrm{~m}, 2 \mathrm{H}), \quad 3.72-$ $3.78(\mathrm{~m}, 2 \mathrm{H}), 3.84-3.90(\mathrm{~m}, 2 \mathrm{H}), 4.11-4.22(\mathrm{~m}, 2 \mathrm{H}), 4.43(\mathrm{~d}, J=6.8 \mathrm{~Hz}, 0.5 \times 1 \mathrm{H}), 4.63(\mathrm{~d}, J=4.0$ $\mathrm{Hz}, 0.5 \times 1 \mathrm{H}), 4.80(\mathrm{~m}, 0.5 \times 1 \mathrm{H}), 5.06(\mathrm{~m}, 0.5 \times 1 \mathrm{H}), 5.21(\mathrm{brs}, 0.5 \times 1 \mathrm{H}), 5.41(\mathrm{brs}, 0.5 \times 1 \mathrm{H}), 6.38$ $(\mathrm{m}, 1 \mathrm{H}), 6.58(\mathrm{~m}, 1 \mathrm{H}), 6.69(\mathrm{~m}, 1 \mathrm{H}), 6.77(\mathrm{~m}, 1 \mathrm{H}), 7.01(\mathrm{~m}, 1 \mathrm{H}), 7.10-7.23(\mathrm{~m}, 8 \mathrm{H}) .{ }^{13} \mathbf{C}$ NMR $(100.5$ $\left.\mathrm{MHz}_{\mathrm{CDCl}}\right) \delta 15.8,15.8,59.0,59.1,63.7,65.1,68.6,69.0,70.0,70.0,70.7,70.7,72.0(\times 2 \mathrm{C}), 76.6$, $77.8,111.9,112.1,112.3,112.9,117.0,117.1,121.9,122.1,126.0,126.2,127.1,127.3,127.4,127.4$, $127.9,128.1(\times 2 \mathrm{C}), 128.3,137.3,137.5,137.6,137.7,137.8,138.2,138.6,140.3,146.3,146.4$. HRMS-DART $(m / z):[\mathrm{M}+\mathrm{H}]^{+}$calcd for $\mathrm{C}_{26} \mathrm{H}_{32} \mathrm{~N}_{1} \mathrm{O}_{4} \mathrm{~S}_{1}, 454.2047$; found, 454.2052. $[\alpha]_{\mathrm{D}}{ }^{29}+4.6(c$ $\left.0.12, \mathrm{CHCl}_{3}\right)$. The ee value $(69 \%$ ee/68\% ee) was determined by chiral HPLC analysis [CHIRALCEL ${ }^{\circledR}$ IC-3 column, $4.6 \mathrm{~mm} \times 250 \mathrm{~mm}$, Daicel Chemical Industries, hexane/2-propanol = 90:10, $0.5 \mathrm{~mL} / \mathrm{min}, 40{ }^{\circ} \mathrm{C}, 250 \mathrm{~nm} \mathrm{UV}$ detector, $\mathrm{t}_{\mathrm{R}}=31.1$ min $\left(\right.$ major $\left._{\mathrm{A}}\right), 33.1$ min $\left(\right.$ minor $\left._{\mathrm{B}}\right), 38.8 \mathrm{~min}$ $\left(\operatorname{minor}_{\mathrm{A}}\right), 64.8 \mathrm{~min}\left(\right.$ major $\left.\left._{\mathrm{B}}\right)\right]$. The absolute configuration of 3 if was assigned by consideration of the stereochemical pathway.

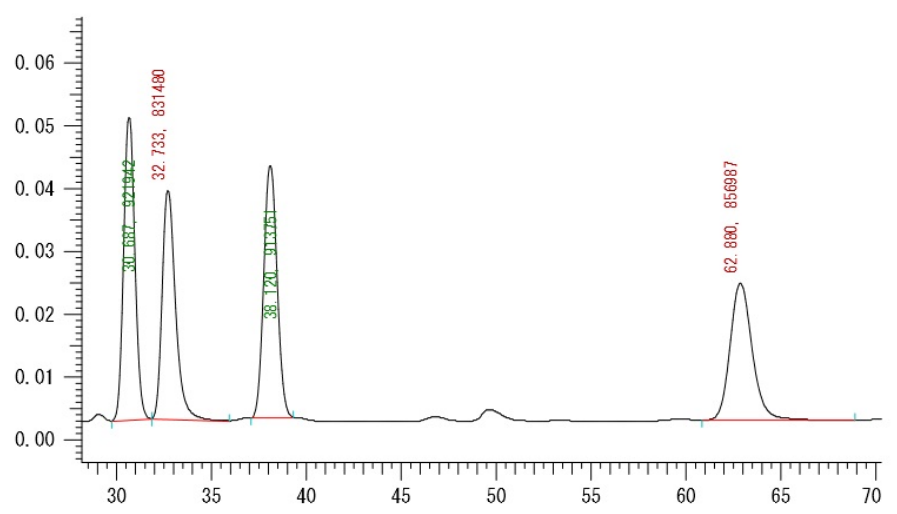

rac-3if

$\begin{array}{llll}\text { No } & \text { rt (min) } & \text { area } & \text { area (\%) } \\ 1 & 30.69 & 921942 & 26.158 \\ 2 & 32.73 & 831786 & 23.600 \\ 3 & 38.12 & 913751 & 25.926 \\ 4 & 62.88 & 856987 & 24.315\end{array}$

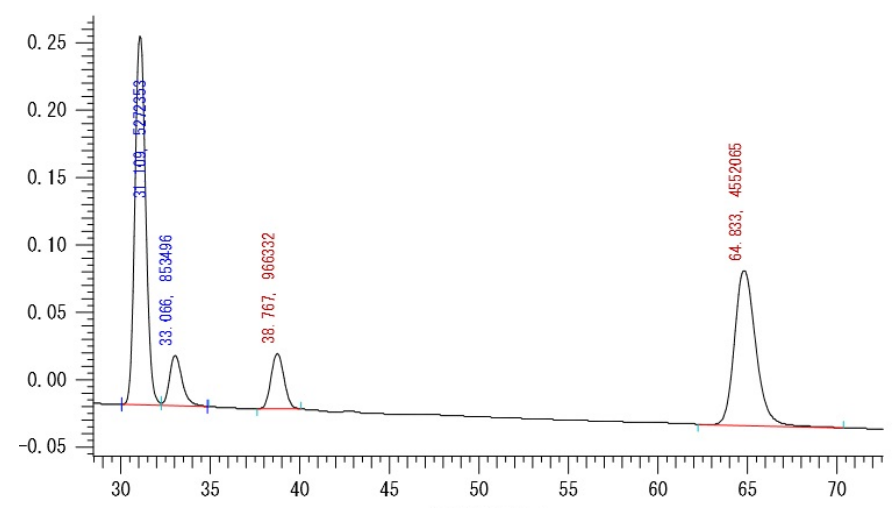

(1S)-3if

$\begin{array}{llll}\text { No } & \text { rt (min) } & \text { area } & \text { area (\%) } \\ 1 & 31.11 & 5272353 & 45.279 \\ 2 & 33.07 & 853496 & 7.330 \\ 3 & 38.77 & 966332 & 8.229 \\ 4 & 64.83 & 4552065 & 39.093\end{array}$




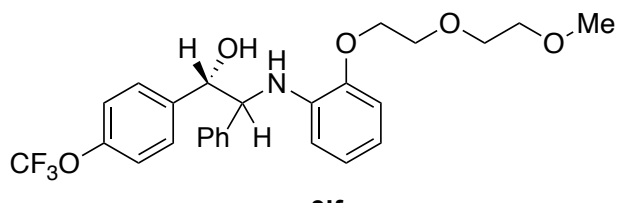

$3 \mathbf{j f}$

The product $3 \mathbf{j}$ f was purified by flash chromatography on silica gel (hexane/Et 3 N/EtOAc 5:0.5:9530:0.5:70) (Scheme 1; $75.9 \mathrm{mg}, 0.154 \mathrm{mmol}, 77 \%$ isolated yield). The diastereomeric ratio (1:1) was determined by ${ }^{1} \mathrm{H}$ NMR. Yellow oil. IR (neat) 738, 1164, 1221, 1259, 1453, 1509, 1602, 2879, 2928, $3063,3409 \mathrm{~cm}^{-1} .{ }^{1} \mathbf{H}$ NMR $\left(600 \mathrm{MHz}, \mathrm{CDCl}_{3}\right) \delta 3.27$ (brs, $\left.0.5 \times 1 \mathrm{H}\right), 3.36(\mathrm{~s}, 0.5 \times 3 \mathrm{H}), 3.37(\mathrm{~s}, 0.5$ $\times 3 \mathrm{H}), 3.54$ (brs, $0.5 \times 1 \mathrm{H}), 3.59-3.63(\mathrm{~m}, 2 \mathrm{H}), 3.71-3.78(\mathrm{~m}, 2 \mathrm{H}), 3.82-3.92(\mathrm{~m}, 2 \mathrm{H}), 4.13-4.20(\mathrm{~m}$, $2 \mathrm{H}), 4.41(\mathrm{~d}, J=6.6 \mathrm{~Hz}, 0.5 \times 1 \mathrm{H}), 4.62(\mathrm{brs}, 0.5 \times 1 \mathrm{H}), 4.84(\mathrm{~m}, 0.5 \times 1 \mathrm{H}), 5.12(\mathrm{t}, J=4.8 \mathrm{~Hz}, 0.5$ $\times 1 \mathrm{H}), 5.26(\mathrm{brs}, 0.5 \times 1 \mathrm{H}), 5.43(\mathrm{brs}, 0.5 \times 1 \mathrm{H}), 6.38(\mathrm{~m}, 1 \mathrm{H}), 6.59(\mathrm{~m}, 1 \mathrm{H}), 6.70(\mathrm{~m}, 1 \mathrm{H}), 6.79(\mathrm{~m}$, 1H), 7.07-7.22 (m, 9H). ${ }^{13} \mathbf{C}$ NMR (150.9 MHz, $\left.\mathrm{CDCl}_{3}\right) \delta 59.0,59.0,63.8,65.3,68.8,69.1,70.0(\times$ 2C), 70.6, 70.6, 72.0 ( $\times 2 \mathrm{C}), 76.0,77.5,112.0,112.2,112.6,113.2,117.1,117.3,120.3,120.4,122.0$, $122.2,127.3,127.5(\times 2 \mathrm{C}), 127.9,128.0,128.1,128.2,128.4,137.5(\times 2 \mathrm{C}), 138.2,138.3,139.3$, $139.6,140.0(\times 2 \mathrm{C}), 146.3,146.4,148.5(\times 2 \mathrm{C})$. HRMS-DART $(\mathrm{m} / \mathrm{z}):[\mathrm{M}+\mathrm{H}]^{+}$calcd for $\mathrm{C}_{26} \mathrm{H}_{29} \mathrm{~F}_{3} \mathrm{~N}_{1} \mathrm{O}_{5}, 492.1992$; found, 492.2001. [ $\left.\alpha\right]_{\mathrm{D}}{ }^{29}+3.6$ (c 1.12, $\left.\mathrm{CHCl}_{3}\right)$. The ee value $(80 \%$ ee/82\% ee) was determined by chiral HPLC analysis [CHIRALCEL ${ }^{\circledR}$ AD-3 column, $4.6 \mathrm{~mm} \times 250 \mathrm{~mm}$, Daicel Chemical Industries, hexane $/ 2$-propanol $=98: 2,1.0 \mathrm{~mL} / \mathrm{min}, 40{ }^{\circ} \mathrm{C}, 250 \mathrm{~nm} \mathrm{UV}$ detector, $\mathrm{t}_{\mathrm{R}}=$ $54.4 \min \left(\right.$ minor $\left._{\mathrm{B}}\right), 57.7 \min \left(\right.$ major $\left._{\mathrm{B}}\right), 64.5 \min \left(\right.$ minor $\left._{\mathrm{A}}\right), 85.8 \min \left(\right.$ major $\left.\left._{\mathrm{A}}\right)\right]$. The absolute configuration of $\mathbf{3 j \mathbf { j }}$ was assigned by consideration of the stereochemical pathway.

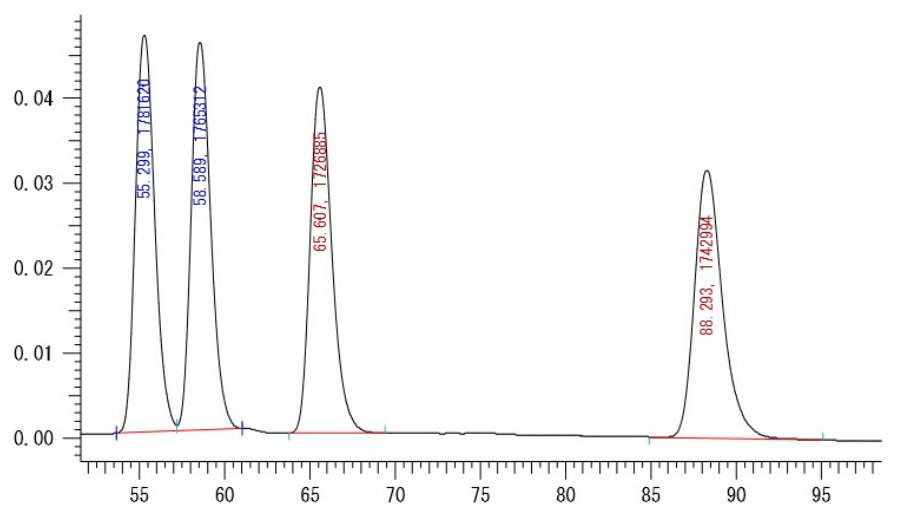

\begin{tabular}{llll}
\multicolumn{2}{l}{$r a c-\mathbf{3 j} \mathbf{f}$} \\
No & rt (min) & area & area (\%) \\
1 & 55.30 & 1781620 & 25.391 \\
2 & 58.59 & 1765312 & 25.158 \\
3 & 65.61 & 1726885 & 24.611 \\
4 & 88.29 & 1742994 & 24.840
\end{tabular}

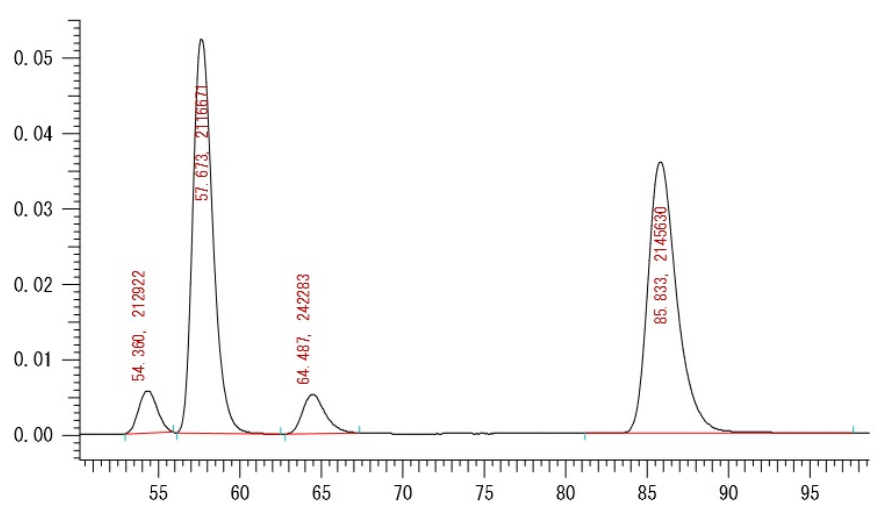

(1S)-3jf

$\begin{array}{llll}\text { No } & \text { rt (min) } & \text { area } & \text { area (\%) } \\ 1 & 54.36 & 212922 & 4.513 \\ 2 & 57.67 & 2116671 & 44.868 \\ 3 & 64.49 & 242283 & 5.136 \\ 4 & 85.83 & 2145630 & 45.482\end{array}$



$(3 \mathbf{k f})$

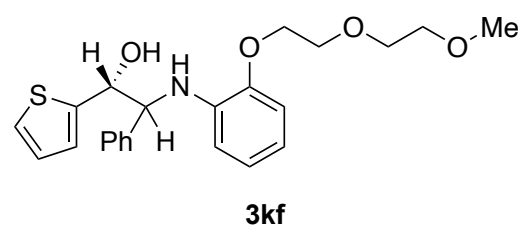

The product 3kf was purified by flash chromatography on silica gel (hexane/Et 3 N/EtOAc 5:0.5:9530:0.5:70) (Scheme 1; $55.7 \mathrm{mg}, 0.135 \mathrm{mmol}, 67 \%$ isolated yield). The diastereomeric ratio (1:1) was determined by ${ }^{1} \mathrm{H}$ NMR. Yellow oil. IR (neat) 702, 1052, 1128, 1217, 1452, 1511, 1601, 2877, 2924 , 3028, 3062, $3403 \mathrm{~cm}^{-1} .{ }^{1} \mathbf{H}$ NMR $\left(400 \mathrm{MHz}, \mathrm{CDCl}_{3}\right) \delta 3.25(\mathrm{~d}, J=6.0 \mathrm{~Hz}, 0.5 \times 1 \mathrm{H}), 3.36(\mathrm{~s}, 0.5 \times$ $3 \mathrm{H}), 3.37(\mathrm{~s}, 0.5 \times 3 \mathrm{H}), 3.47(\mathrm{brs}, 0.5 \times 1 \mathrm{H}), 3.57-3.60(\mathrm{~m}, 2 \mathrm{H}), 3.71-3.77(\mathrm{~m}, 2 \mathrm{H}), 3.84-3.90(\mathrm{~m}$, $2 \mathrm{H}), 4.11-4.21(\mathrm{~m}, 2 \mathrm{H}), 4.55(\mathrm{~d}, J=6.0 \mathrm{~Hz}, 0.5 \times 1 \mathrm{H}), 4.71(\mathrm{~d}, J=3.6 \mathrm{~Hz}, 0.5 \times 1 \mathrm{H}), 5.14(\mathrm{dd}, J=$ 6.0, $2.4 \mathrm{~Hz}, 0.5 \times 1 \mathrm{H}), 5.21$ (brs, $0.5 \times 1 \mathrm{H}), 5.33(\mathrm{~m}, 0.5 \times 1 \mathrm{H}), 5.49$ (brs, $0.5 \times 1 \mathrm{H}), 6.42(\mathrm{~m}, 1 \mathrm{H})$, $6.60(\mathrm{~m}, 1 \mathrm{H}), 6.69-6.92(\mathrm{~m}, 4 \mathrm{H}), 7.18-7.26(\mathrm{~m}, 6 \mathrm{H}) .{ }^{13} \mathbf{C ~ N M R}\left(100.5 \mathrm{MHz}, \mathrm{CDCl}_{3}\right) \delta 59.0,59.1$, $63.9,64.8,68.7,69.2(\times 2 \mathrm{C}), 70.0,70.6,70.7,72.0,72.0,73.7,74.4,111.9,112.1,112.2,113.0,117.2$ ( $\times$ 2C) $121.9,122.2,124.6,124.7,124.9,125.2,126.2,126.5,127.3,127.4,127.5,127.7,128.3$, $128.4,137.5,138.0,138.8,140.3,144.2,144.4,146.4,146.5$. HRMS-DART $(\mathrm{m} / z):[\mathrm{M}+\mathrm{H}]^{+}$calcd for $\mathrm{C}_{23} \mathrm{H}_{28} \mathrm{~N}_{1} \mathrm{O}_{4} \mathrm{~S}_{1}, 414.1734$; found, 414.1730. $[\alpha]_{\mathrm{D}}^{29}+4.5$ (c 1.00, $\left.\mathrm{CHCl}_{3}\right)$. The ee value $(78 \%$ ee/80\% ee) was determined by chiral HPLC analysis [CHIRALCEL ${ }^{\circledR}$ AD-3 column, $4.6 \mathrm{~mm} \times 250$ $\mathrm{mm}$, Daicel Chemical Industries, hexane/2-propanol = 95:5, $1.0 \mathrm{~mL} / \mathrm{min}, 40{ }^{\circ} \mathrm{C}, 250 \mathrm{~nm} \mathrm{UV}$ detector,

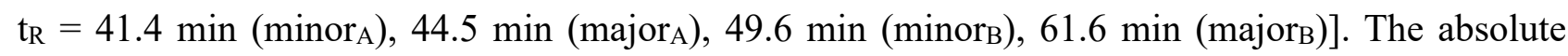
configuration of $\mathbf{3 k f}$ was assigned by consideration of the stereochemical pathway.

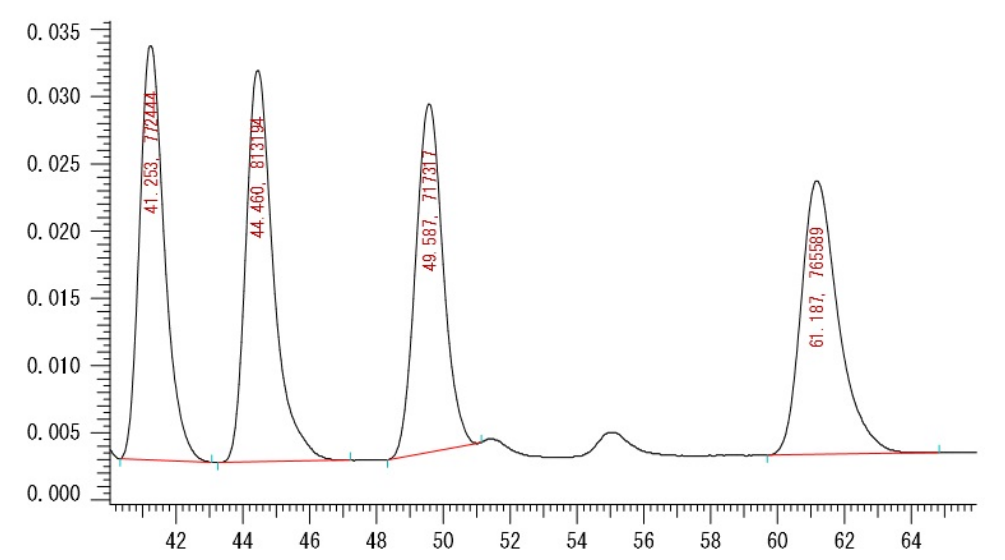

\begin{tabular}{llll}
\multicolumn{2}{l}{$r a c-3 \mathbf{k}$} \\
No & rt (min) & area & area (\%) \\
1 & 41.25 & 772444 & 25.173 \\
2 & 44.46 & 813194 & 26.501 \\
3 & 49.59 & 717317 & 23.376 \\
4 & 61.19 & 765589 & 24.950
\end{tabular}

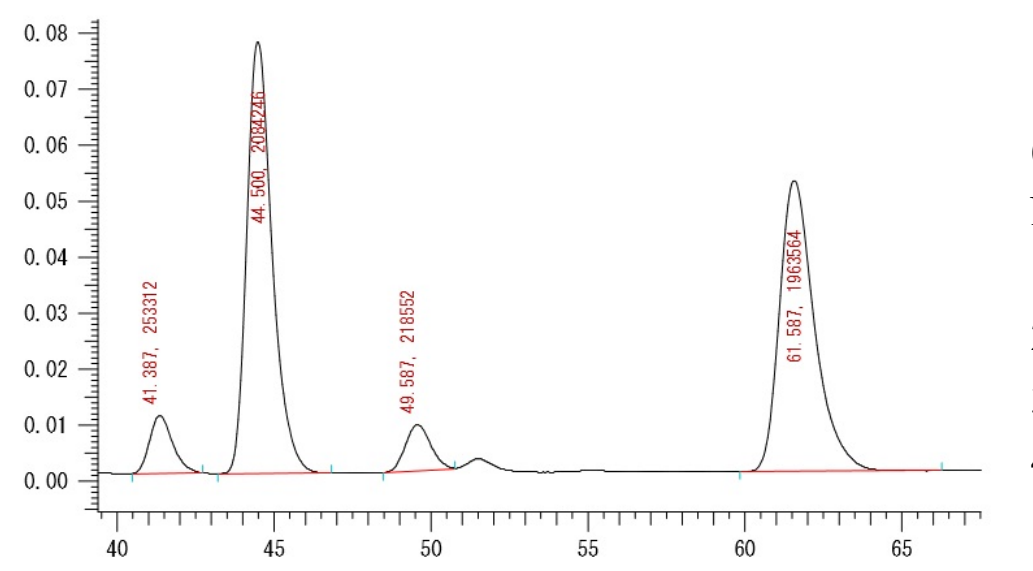

(1S)-3kf

$\begin{array}{llll}\text { No } & \text { rt (min) } & \text { area } & \text { area }(\%) \\ 1 & 41.39 & 253312 & 5.605 \\ 2 & 44.50 & 2084246 & 46.115 \\ 3 & 49.59 & 218552 & 4.836 \\ 4 & 61.59 & 1963564 & 43.445\end{array}$




\section{1-ol (3cg)}

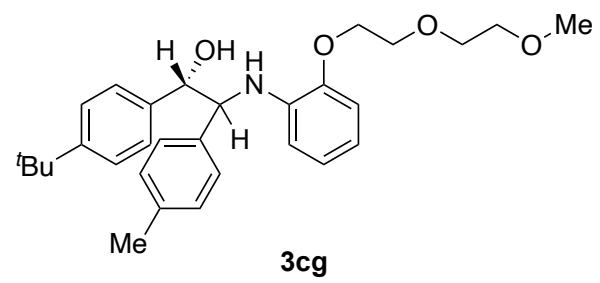

The product $3 \mathrm{cg}$ was purified by flash chromatography on silica gel (hexane/Et ${ }_{3} \mathrm{~N} / \mathrm{EtOAc}$ 5:0.5:9530:0.5:70) (Scheme 1; $84.8 \mathrm{mg}, 0.178 \mathrm{mmol}, 89 \%$ isolated yield). The diastereomeric ratio (1:1) is determined by ${ }^{1} \mathrm{H}$ NMR. Yellow oil. IR (neat) 736, 1051, 1109, 1214, 1443, 1511, 1602, 2871, 2902 , 2926, 2961, 3025, 3046, $3421 \mathrm{~cm}^{-1} .{ }^{1} \mathbf{H}$ NMR (600 MHz, $\left.\mathrm{CDCl}_{3}\right) \delta 1.29(\mathrm{~s}, 0.5 \times 9 \mathrm{H}), 1.30(\mathrm{~s}, 0.5 \times$ $9 \mathrm{H}), 2.28(\mathrm{~s}, 0.5 \times 3 \mathrm{H}), 2.30(\mathrm{~s}, 0.5 \times 3 \mathrm{H}), 2.65(\mathrm{~m}, 0.5 \times 1 \mathrm{H}), 2.97(\mathrm{brs}, 0.5 \times 1 \mathrm{H}), 3.38(\mathrm{~s}, 0.5 \times$ $3 \mathrm{H}), 3.39(\mathrm{~s}, 0.5 \times 3 \mathrm{H}) 3.59-3.61(\mathrm{~m}, 2 \mathrm{H}), 3.73-3.90(\mathrm{~m}, 4 \mathrm{H}), 4.05-4.19(\mathrm{~m}, 2 \mathrm{H}), 4.46(\mathrm{~d}, J=5.4$ $\mathrm{Hz}, 0.5 \times 1 \mathrm{H}), 4.56(\mathrm{~d}, J=4.8 \mathrm{~Hz}, 0.5 \times 1 \mathrm{H}), 4.84(\mathrm{~d}, J=5.4 \mathrm{~Hz}, 0.5 \times 1 \mathrm{H}), 4.97(\mathrm{t}, J=4.2 \mathrm{~Hz}, 0.5$ $\times 1 \mathrm{H}), 5.09$ (brs, $0.5 \times 1 \mathrm{H}), 5.39$ (brs, $0.5 \times 1 \mathrm{H}), 6.33(\mathrm{~m}, 1 \mathrm{H}), 6.54(\mathrm{~m}, 1 \mathrm{H}), 6.66(\mathrm{~m}, 1 \mathrm{H}), 6.75(\mathrm{~m}$, 1H), 7.01-7.12 (m, 5H), $7.20(\mathrm{~m}, 1 \mathrm{H}), 7.27-7.30(\mathrm{~m}, 2 \mathrm{H}) .{ }^{13} \mathbf{C} \mathbf{N M R}\left(150.9 \mathrm{MHz}, \mathrm{CDCl}_{3}\right) \delta 21.1$, 21.1, 31.3, 31.3, 34.4, 34.5, 59.0, 59.0, 63.5, 64.2, 68.4, 68.8, 69.9, 69.9, 70.7, 70.7, 72.0, 72.0, 77.1, $77.9,111.7,111.9,111.9,112.4,116.6(\times 2 \mathrm{C}), 121.8,121.9,124.9,125.0,126.4(\times 2 \mathrm{C}), 127.1,127.7$, 128.9, 129.0, 136.1, 136.7, 136.9, 137.5, 137.5, 137.6, 138.0, 138.1, 146.1, 146.3, 150.5, 150.6. HRMS-DART $(\mathrm{m} / \mathrm{z}):[\mathrm{M}+\mathrm{H}]^{+}$calcd for $\mathrm{C}_{30} \mathrm{H}_{40} \mathrm{~N}_{1} \mathrm{O}_{4}, 478.2952$; found, 478.2952. $[\alpha]_{\mathrm{D}}{ }^{29}+5.0(\mathrm{c} 1.03$, $\left.\mathrm{CHCl}_{3}\right)$. The ee value $\left(89 \%\right.$ ee/89\% ee) was determined by chiral HPLC analysis [CHIRALCEL ${ }^{\circledR}$ AD-3 + AD-H column, $4.6 \mathrm{~mm} \times 250 \mathrm{~mm}$, Daicel Chemical Industries, hexane/2-propanol = 98:2, $1.0 \mathrm{~mL} / \mathrm{min}, 40{ }^{\circ} \mathrm{C}, 250 \mathrm{~nm} \mathrm{UV}$ detector, $\mathrm{t}_{\mathrm{R}}=131.3 \mathrm{~min}$ (major $\left.{ }_{\mathrm{B}}\right), 136.6 \mathrm{~min}\left(\right.$ major $\left._{\mathrm{A}}\right), 178.7 \mathrm{~min}$ $\left(\right.$ minor $\left.\left._{\mathrm{B}}\right), 184.8 \mathrm{~min}\left(\operatorname{minor}_{\mathrm{A}}\right)\right]$. The absolute configuration of $\mathbf{3} \mathbf{c g}$ was assigned by consideration of the stereochemical pathway.

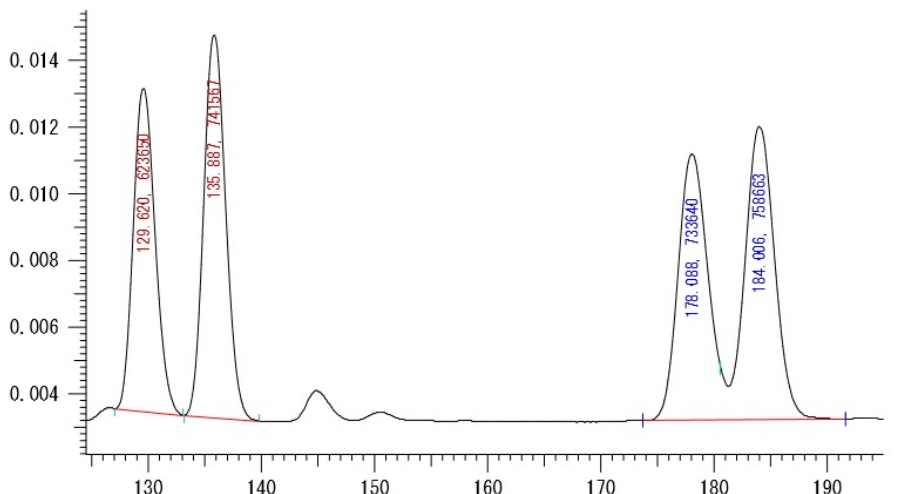

$\begin{array}{llll}r a c-3 c g & & \\ \text { No } & \text { rt (min) } & \text { area } & \text { area (\%) } \\ 1 & 129.62 & 623650 & 21.825 \\ 2 & 135.89 & 741567 & 25.951 \\ 3 & 178.09 & 733640 & 25.674 \\ 4 & 184.01 & 758663 & 26.550\end{array}$

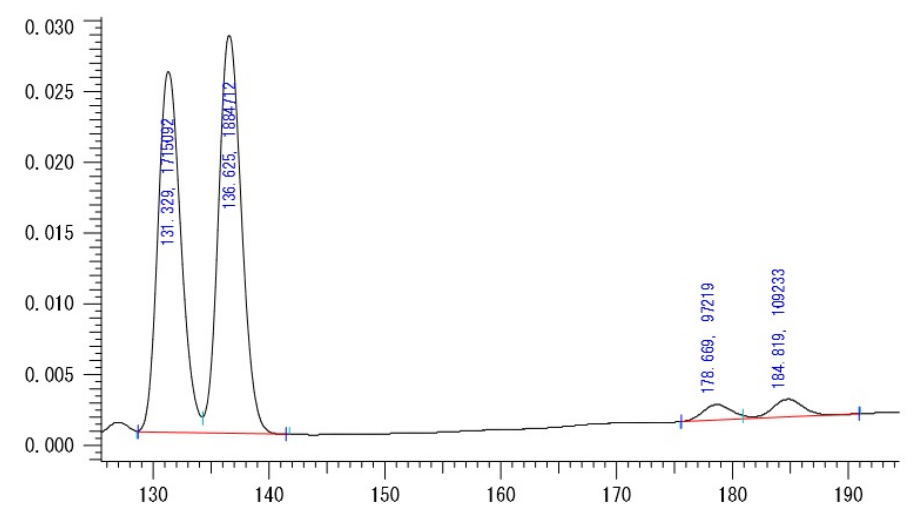

\section{$(1 S)-\mathbf{3 c g}$}

$\begin{array}{llll}\text { No } & \text { rt (min) } & \text { area } & \text { area }(\%) \\ 1 & 131.33 & 1715092 & 45.060 \\ 2 & 136.63 & 1884712 & 49.516 \\ 3 & 178.67 & 97219 & 2.554 \\ 4 & 184.82 & 109233 & 2.870\end{array}$



(3ch)

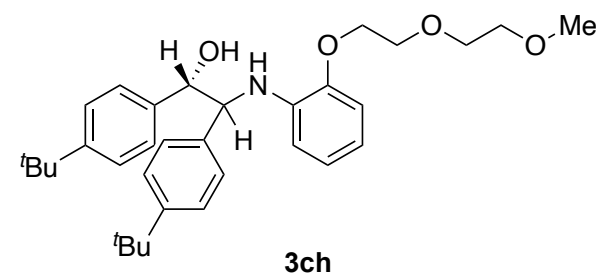

The product 3ch was purified by flash chromatography on silica gel (hexane/Et ${ }_{3} \mathrm{~N} / \mathrm{EtOAc}$ 5:0.5:9530:0.5:70) (Scheme 1; $102.9 \mathrm{mg}, 0.198 \mathrm{mmol}, 99 \%$ isolated yield). The diastereomeric ratio (1:1) was determined by ${ }^{1} \mathrm{H}$ NMR. Yellow oil. IR (neat) 735, 1051, 1108, 1246, 1443, 1510, 1602, 2869, 2902, 2960, 3055, $3420 \mathrm{~cm}^{-1} .{ }^{1} \mathbf{H}$ NMR $\left(600 \mathrm{MHz} \mathrm{CDCl}_{3}\right) \delta 1.27(\mathrm{~s}, 0.5 \times 9 \mathrm{H}), 1.28(\mathrm{~s}, 0.5 \times 9 \mathrm{H})$, $1.29(\mathrm{~s}, 0.5 \times 9 \mathrm{H}), 1.30(\mathrm{~s}, 0.5 \times 9 \mathrm{H}), 2.59(\mathrm{~m}, 0.5 \times 1 \mathrm{H}), 2.86(\mathrm{brs}, 0.5 \times 1 \mathrm{H}), 3.37(\mathrm{~s}, 0.5 \times 3 \mathrm{H})$, $3.39(\mathrm{~s}, 0.5 \times 3 \mathrm{H}), 3.59-3.61(\mathrm{~m}, 2 \mathrm{H}), 3.73-3.90(\mathrm{~m}, 4 \mathrm{H}), 4.03-4.19(\mathrm{~m}, 2 \mathrm{H}), 4.48(\mathrm{~d}, J=5.4 \mathrm{~Hz}, 0.5$ $\times 1 \mathrm{H}), 4.54(\mathrm{~d}, J=5.4 \mathrm{~Hz}, 0.5 \times 1 \mathrm{H}), 4.87(\mathrm{~d}, J=3.6 \mathrm{~Hz}, 0.5 \times 1 \mathrm{H}), 4.93(\mathrm{t}, J=5.4 \mathrm{~Hz}, 0.5 \times 1 \mathrm{H})$, 5.03 (brs, $0.5 \times 1 \mathrm{H}), 5.40$ (brs, $0.5 \times 1 \mathrm{H}), 6.35(\mathrm{~m}, 1 \mathrm{H}), 6.54(\mathrm{~m}, 1 \mathrm{H}), 6.66-6.77(\mathrm{~m}, 2 \mathrm{H}), 7.11-7.16$ (m, 3H), 7.22-7.32 (m, 5H). ${ }^{13} \mathbf{C}$ NMR (150.9 MHz, $\left.\mathrm{CDCl}_{3}\right) \delta 31.3(\times 4 \mathrm{C}), 34.4,34.4,34.4,34.5$, 59.0, 59.1, 63.5, 63.8, 68.4, 68.8, 69.9, 69.9, 70.7 (× 2C), 72.0, 72.0, 77.2, 77.8, 111.6, 111.7, 111.9, 112.4, 116.4, 116.6, 121.8, 121.9, 124.9, 124.9, 125.2, 125.2, 126.2, 126.4, 126.7, 127.3, 136.2, 137.5, 137.6, 137.7, 138.1, 138.2, 146.1, 146.2, 150.0, 150.2, 150.4, 150.6. HRMS-DART $(\mathrm{m} / \mathrm{z}):[\mathrm{M}+\mathrm{H}]^{+}$ calcd for $\mathrm{C}_{33} \mathrm{H}_{46} \mathrm{~N}_{1} \mathrm{O}_{4}, 520.3421$; found, 520.3414. $[\alpha]_{\mathrm{D}}{ }^{29}+4.8\left(c 0.94, \mathrm{CHCl}_{3}\right)$. The ee value $(88 \%$ ee/93\% ee) was determined by chiral HPLC analysis [CHIRALCEL ${ }^{\circledR}$ AD-3 column, $4.6 \mathrm{~mm} \times 250$ $\mathrm{mm}$, Daicel Chemical Industries, hexane/2-propanol = 95:5, $0.5 \mathrm{~mL} / \mathrm{min}, 40{ }^{\circ} \mathrm{C}, 250 \mathrm{~nm} \mathrm{UV}$ detector, $t_{\mathrm{R}}=29.4 \min \left(\right.$ major $\left._{\mathrm{B}}\right), 35.2 \min \left(\operatorname{minor}_{\mathrm{A}}\right), 38.1 \min \left(\right.$ minor $\left._{\mathrm{B}}\right), 44.6 \min \left(\right.$ major $\left.\left._{\mathrm{A}}\right)\right]$. The absolute configuration of $\mathbf{3 c h}$ was assigned by consideration of the stereochemical pathway.

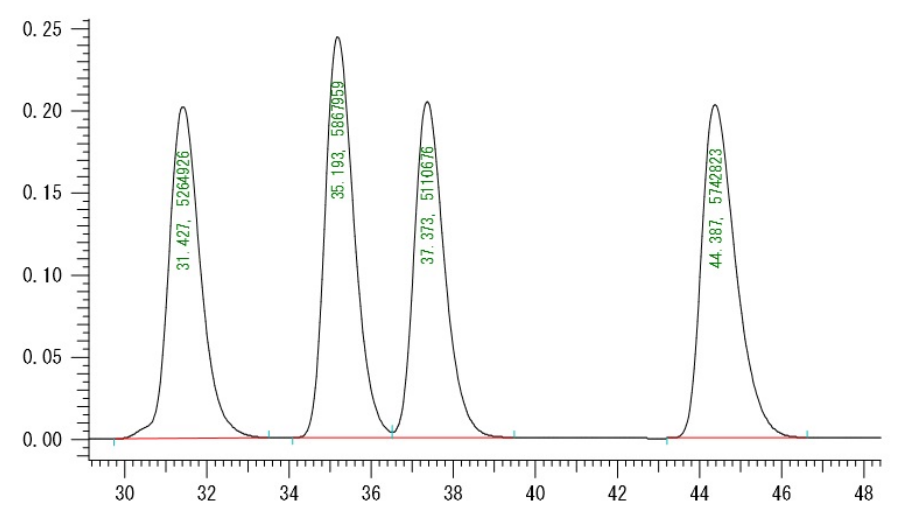

\begin{tabular}{llll}
\multicolumn{2}{l}{$r a c-3 c h$} \\
No & rt (min) & area & area (\%) \\
1 & 31.43 & 5264926 & 23.946 \\
2 & 35.19 & 5867959 & 26.689 \\
3 & 37.37 & 5110676 & 23.245 \\
4 & 44.39 & 5742823 & 26.120
\end{tabular}

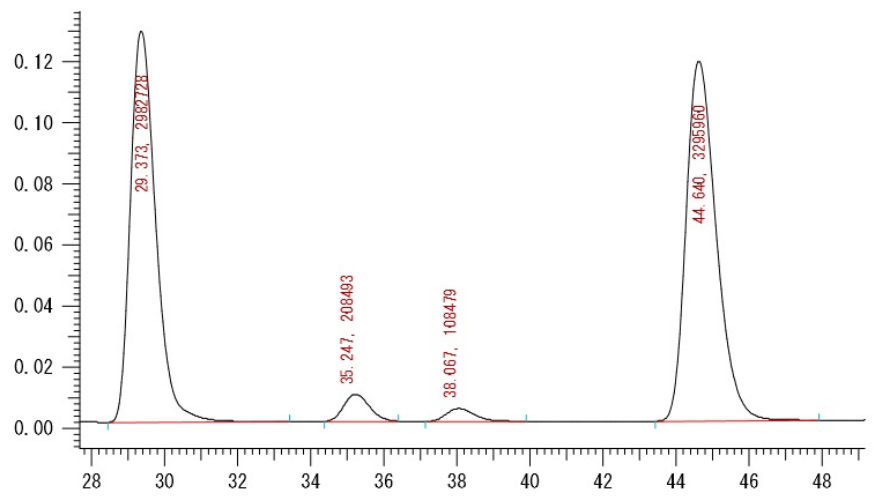

$(1 S)-\mathbf{3 c h}$

$\begin{array}{llll}\text { No } & \text { rt (min) } & \text { area } & \text { area (\%) } \\ 1 & 29.37 & 2982728 & 45.223 \\ 2 & 35.25 & 208493 & 3.161 \\ 3 & 38.07 & 108479 & 1.645 \\ 4 & 44.64 & 3295960 & 49.972\end{array}$




\section{1-ol (3ci)}

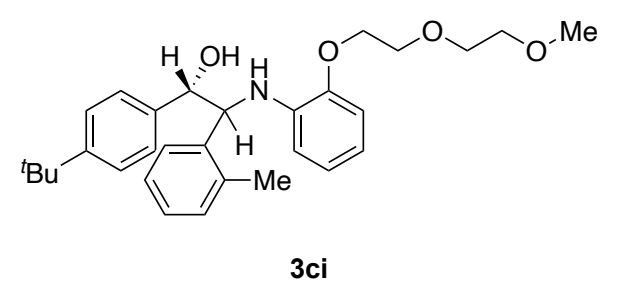

The product 3ci was purified by flash chromatography on silica gel (hexane/Et 3 N/EtOAc 5:0.5:9530:0.5:70) (Scheme 1; $82.8 \mathrm{mg}, 0.173 \mathrm{mmol}, 87 \%$ isolated yield). The diastereomeric ratio (1:1) was determined by ${ }^{1} \mathrm{H}$ NMR. Yellow oil. IR (neat) 734, 1108, 1215, 1459, 1510, 1601, 2871, 2902, 2960, 3025, 3062, $3415 \mathrm{~cm}^{-1} .{ }^{1} \mathbf{H}$ NMR $\left(400 \mathrm{MHz} \mathrm{CDCl}_{3}\right) \delta 1.28(\mathrm{~s}, 0.5 \times 9 \mathrm{H}), 1.29(\mathrm{~s}, 0.5 \times 9 \mathrm{H}), 2.04(\mathrm{~s}$, $0.5 \times 3 \mathrm{H}), 2.17(\mathrm{~s}, 0.5 \times 3 \mathrm{H}), 2.69(\mathrm{~m}, 0.5 \times 1 \mathrm{H}), 3.07(\mathrm{brs}, 0.5 \times 1 \mathrm{H}), 3.38(\mathrm{~s}, 0.5 \times 3 \mathrm{H}), 3.39(\mathrm{~s}, 0.5$ $\times 3 \mathrm{H}), 3.59-3.63(\mathrm{~m}, 2 \mathrm{H}), 3.74-3.80(\mathrm{~m}, 2 \mathrm{H}), 3.84-3.91(\mathrm{~m}, 2 \mathrm{H}), 4.08-4.19(\mathrm{~m}, 2 \mathrm{H}), 4.68(\mathrm{~d}, J=5.6$ $\mathrm{Hz}, 0.5 \times 1 \mathrm{H}), 4.84(\mathrm{~m}, 2 \mathrm{H}), 5.00(\mathrm{t}, J=5.2 \mathrm{~Hz}, 0.5 \times 1 \mathrm{H}), 5.15$ (brs, $0.5 \times 1 \mathrm{H}), 5.48(\mathrm{brs}, 0.5 \times 1 \mathrm{H})$, $6.24(\mathrm{~m}, 1 \mathrm{H}), 6.54(\mathrm{~m}, 1 \mathrm{H}), 6.66(\mathrm{~m}, 1 \mathrm{H}), 6.74(\mathrm{~s}, 1 \mathrm{H}), 7.02-7.28(\mathrm{~m}, 8 \mathrm{H}) .{ }^{13} \mathrm{C}$ NMR $(100.5 \mathrm{MHz}$, $\left.\mathrm{CDCl}_{3}\right) \delta 19.0,19.2,31.3,31.3,34.4,34.5,59.0,59.1(\times 2 \mathrm{C}), 59.3,60.4(\times 2 \mathrm{C}), 68.5,68.9,69.9,70.0$, $70.8(\times 2 \mathrm{C}), 72.0(\times 2 \mathrm{C}), 111.3,111.5,112.0,112.6,116.7,116.7,121.9,122.0,124.8,124.9,126.0$, 126.1, 126.3, 126.4, 126.5, 127.0, 127.1, 127.1, 130.1, 130.4, 135.9, 136.7, 137.2, 137.4, 137.6, 137.9, 138.2, 138.9, 146.2, 146.4, 150.5, 150.9. HRMS-DART $(\mathrm{m} / \mathrm{z}):[\mathrm{M}+\mathrm{H}]^{+}$calcd for $\mathrm{C}_{30} \mathrm{H}_{40} \mathrm{~N}_{1} \mathrm{O}_{4}$, 478.2952; found, 478.2956. $\left.[\alpha]_{\mathrm{D}}{ }^{29}+9.6(c) 0.80, \mathrm{CHCl}_{3}\right)$. The ee value $(89 \%$ ee/89\% ee) was determined by chiral HPLC analysis [CHIRALCEL ${ }^{\circledR}$ AD-3 column, $4.6 \mathrm{~mm} \times 250 \mathrm{~mm}$, Daicel Chemical Industries, hexane/2-propanol $=93: 7,0.5 \mathrm{~mL} / \mathrm{min}, 40{ }^{\circ} \mathrm{C}, 250 \mathrm{~nm} \mathrm{UV}$ detector, $\mathrm{t}_{\mathrm{R}} 22.2 \mathrm{~min}$ (major $\mathrm{A}), 29.4 \min \left(\right.$ major $\left._{\mathrm{B}}\right), 34.0 \mathrm{~min}\left(\operatorname{minor}_{\mathrm{B}}\right), 39.9 \min \left(\right.$ minor $\left.\left._{\mathrm{A}}\right)\right]$. The absolute configuration of 3ci was assigned by consideration of the stereochemical pathway.

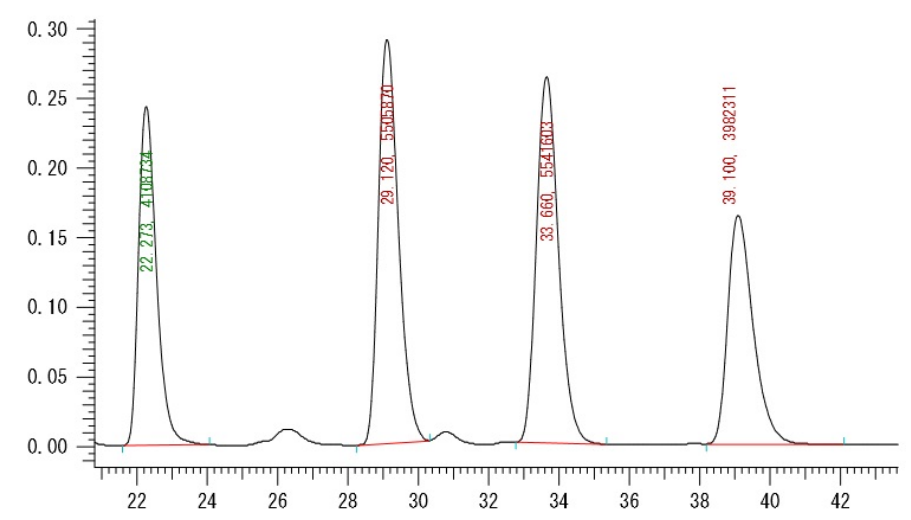

\begin{tabular}{llll}
\multicolumn{2}{l}{$r a c-3 c i$} \\
No & rt (min) & area & area (\%) \\
1 & 22.27 & 4108734 & 21.468 \\
2 & 29.12 & 5505870 & 28.769 \\
3 & 33.66 & 5541603 & 28.955 \\
4 & 39.10 & 3982311 & 20.808
\end{tabular}

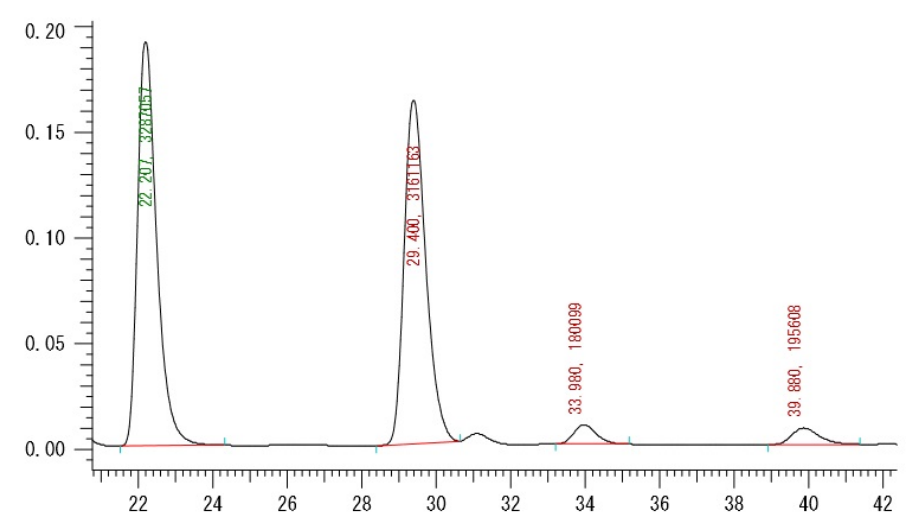

(1S)-3ci

$\begin{array}{llll}\text { No } & \text { rt (min) } & \text { area } & \text { area }(\%) \\ 1 & 22.21 & 3287057 & 48.170 \\ 2 & 29.40 & 3161163 & 46.325 \\ 3 & 33.98 & 180099 & 2.639 \\ 4 & 39.88 & 195608 & 2.867\end{array}$




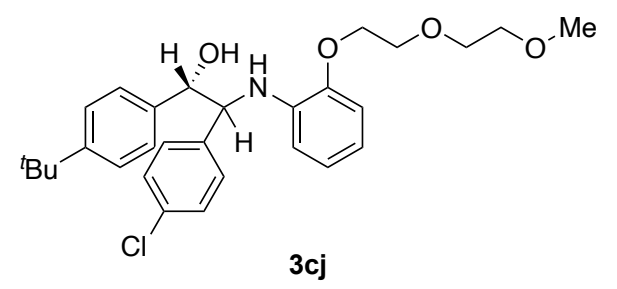

The product 3cj was purified by flash chromatography on silica gel (hexane/Et 3 N/EtOAc 5:0.5:9530:0.5:70) (Scheme 1; $89.4 \mathrm{mg}, 0.179 \mathrm{mmol}, 90 \%$ isolated yield). The diastereomeric ratio (1:1) was determined by ${ }^{1} \mathrm{H}$ NMR. Yellow oil. IR (neat) 736, 1052, 1107, 1215, 1443, 1510, 1602, 2871, 2902 , 2929, 2961, 3063, $3410 \mathrm{~cm}^{-1}{ }^{1}{ }^{1} \mathbf{H}$ NMR $\left(600 \mathrm{MHz} \mathrm{CDCl}_{3}\right) \delta 1.29(\mathrm{~s}, 0.5 \times 9 \mathrm{H}), 1.30(\mathrm{~s}, 0.5 \times 9 \mathrm{H})$, $2.97(\mathrm{~m}, 0.5 \times 1 \mathrm{H}), 3.22(\mathrm{~m}, 0.5 \times 1 \mathrm{H}), 3.359(\mathrm{~s}, 0.5 \times 3 \mathrm{H}), 3.361(\mathrm{~s}, 0.5 \times 3 \mathrm{H}), 3.58-3.61(\mathrm{~m}, 2 \mathrm{H})$, 3.71-3.77 (m, 2H), 3.81-3.89 (m, 2H), 4.08-4.20 (m, 2H), $4.43(\mathrm{~d}, J=6.6 \mathrm{~Hz}, 0.5 \times 1 \mathrm{H}), 4.56(\mathrm{~d}, J$ $=3.0 \mathrm{~Hz}, 0.5 \times 1 \mathrm{H}), 4.76(\mathrm{~d}, J=4.8 \mathrm{~Hz}, 0.5 \times 1 \mathrm{H}), 5.04(\mathrm{~m}, 0.5 \times 1 \mathrm{H}), 5.27(\mathrm{brs}, 0.5 \times 1 \mathrm{H}), 5.48$ (brs, $0.5 \times 1 \mathrm{H}), 6.28(\mathrm{~m}, 1 \mathrm{H}), 6.58(\mathrm{~m}, 1 \mathrm{H}), 6.67(\mathrm{~m}, 1 \mathrm{H}), 6.77(\mathrm{~m}, 1 \mathrm{H}), 7.03-7.17(\mathrm{~m}, 6 \mathrm{H}), 7.26-$ 7.29 (m, 2H). ${ }^{13} \mathbf{C}$ NMR (150.9 MHz, $\left.\mathrm{CDCl}_{3}\right) \delta 31.3,31.3,34.5,34.5,59.0,59.0,63.2,64.3,68.7$, 69.0, $69.9(\times 2 \mathrm{C}), 70.6,70.7,72.0(\times 2 \mathrm{C}), 76.6,77.8,111.7,112.0,112.5,112.8,116.9,117.1,121.9$, $122.0,125.0,125.0,126.2,126.5,128.0,128.3,128.8,129.4,132.8,132.8,137.3,137.5,137.6,137.6$, 138.0, 139.3, 146.2, 146.4, 150.7, 150.8. HRMS-DART $(\mathrm{m} / \mathrm{z}):[\mathrm{M}+\mathrm{H}]^{+}$calcd for $\mathrm{C}_{29} \mathrm{H}_{37} \mathrm{Cl}_{1} \mathrm{~N}_{1} \mathrm{O}_{4}$, 498.2406 ; found, 498.2405. $[\alpha]_{\mathrm{D}}{ }^{29}+4.3\left(c\right.$ 1.61, $\left.\mathrm{CHCl}_{3}\right)$. The ee value $(90 \%$ ee $/ 90 \%$ ee $)$ was determined by chiral HPLC analysis [CHIRALCEL ${ }^{\circledR}$ IC-3 column, $4.6 \mathrm{~mm} \times 250 \mathrm{~mm}$, Daicel Chemical Industries, hexane/2-propanol $=95: 5,0.5 \mathrm{~mL} / \mathrm{min}, 40{ }^{\circ} \mathrm{C}, 250 \mathrm{~nm} \mathrm{UV}$ detector, $\mathrm{t}_{\mathrm{R}} 23.6 \mathrm{~min}$ (major $\mathrm{B}), 29.8 \mathrm{~min}\left(\operatorname{minor}_{\mathrm{A}}\right), 35.0 \mathrm{~min}\left(\right.$ minor $\left._{\mathrm{B}}\right), 70.6 \mathrm{~min}\left(\right.$ major $\left.\left._{\mathrm{A}}\right)\right]$. The absolute configuration of $\mathbf{3 c j}$ was assigned by consideration of the stereochemical pathway.

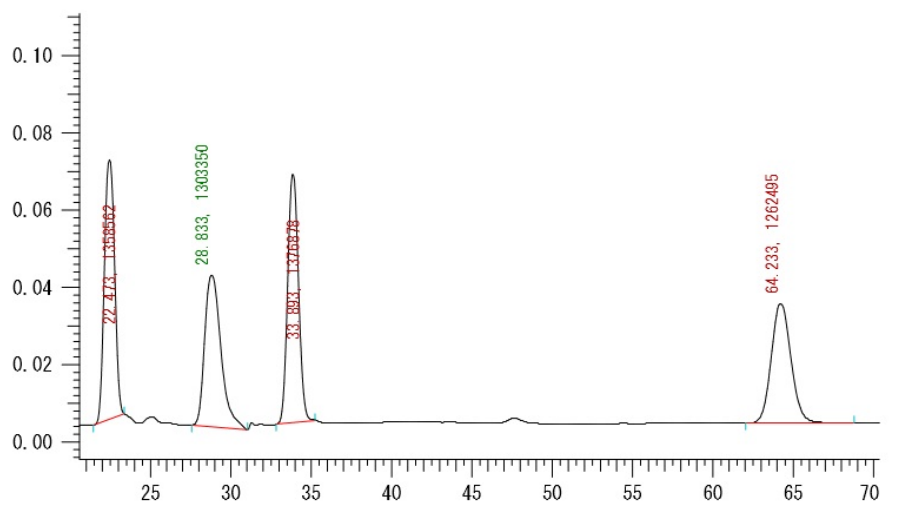

$$
r a c-3 c j
$$

$\begin{array}{llll}\text { No } & \text { rt (min) } & \text { area } & \text { area }(\%) \\ 1 & 22.47 & 1358562 & 25.627 \\ 2 & 28.83 & 1303350 & 24.586 \\ 3 & 33.89 & 1376878 & 25.973 \\ 4 & 64.23 & 1262495 & 23.815\end{array}$

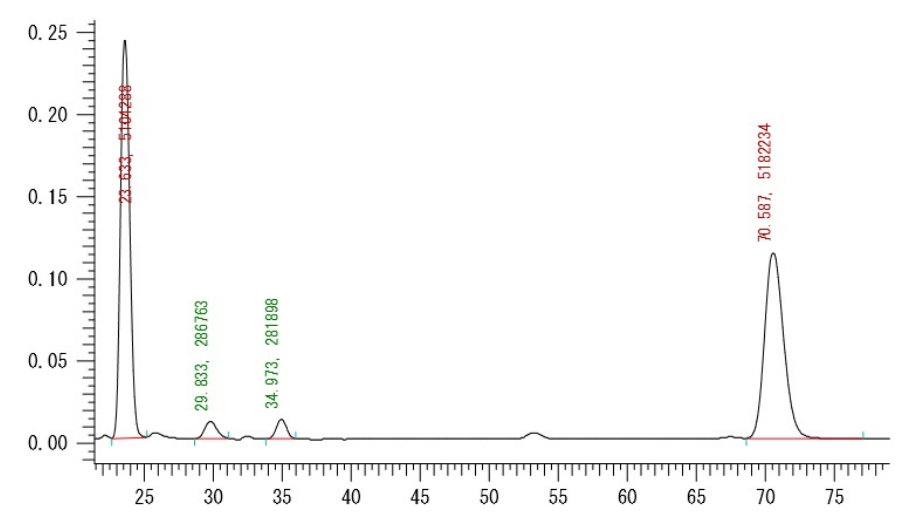

(1S)-3cj

$\begin{array}{llll}\text { No } & \text { rt (min) } & \text { area } & \text { area (\%) } \\ 1 & 23.63 & 5104288 & 47.022 \\ 2 & 29.83 & 286763 & 2.642 \\ 3 & 34.97 & 281898 & 2.597 \\ 4 & 70.59 & 5182234 & 47.740\end{array}$




\section{2-yl)ethan-1-ol (3ck)}

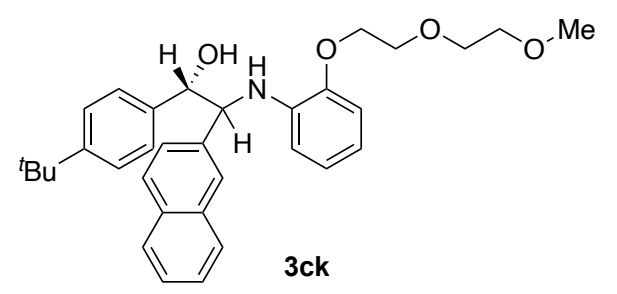

The product 3ck was purified by flash chromatography on silica gel (hexane/Et 3 N/EtOAc 5:0.5:9530:0.5:70) (Scheme 1; $89.4 \mathrm{mg}, 0.174 \mathrm{mmol}, 87 \%$ isolated yield). The diastereomeric ratio (1:1) was determined by ${ }^{1} \mathrm{H}$ NMR. Yellow oil. IR (neat) 739, 1053, 1130, 1215, 1443, 1510, 1601, 2871, 2902 , 2960, 3055, $3417 \mathrm{~cm}^{-1} .{ }^{1} \mathbf{H}$ NMR (600 MHz, $\left.\mathrm{CDCl}_{3}\right) \delta 1.27(\mathrm{~s}, 0.5 \times 9 \mathrm{H}), 1.29(\mathrm{~s}, 0.5 \times 9 \mathrm{H}), 2.76$ (brs, $0.5 \times 1 \mathrm{H}), 3.11($ brs, $0.5 \times 1 \mathrm{H}), 3.37(\mathrm{~s}, 0.5 \times 3 \mathrm{H}), 3.38(\mathrm{~s}, 0.5 \times 3 \mathrm{H}), 3.61-3.63(\mathrm{~m}, 2 \mathrm{H}), 3.76-$ $3.95(\mathrm{~m}, 4 \mathrm{H}), 4.09-4.23(\mathrm{~m}, 2 \mathrm{H}), 4.63(\mathrm{~d}, J=6.0 \mathrm{~Hz}, 0.5 \times 1 \mathrm{H}), 4.74(\mathrm{brs}, 0.5 \times 1 \mathrm{H}), 4.93(\mathrm{~m}, 0.5 \times$ $1 \mathrm{H}), 5.11(\mathrm{~m}, 0.5 \times 1 \mathrm{H}), 5.28(\mathrm{brs}, 0.5 \times 1 \mathrm{H}), 5.56(\mathrm{brs}, 0.5 \times 1 \mathrm{H}), 6.35(\mathrm{~m}, 1 \mathrm{H}), 6.54(\mathrm{~m}, 1 \mathrm{H}), 6.62$ $(\mathrm{m}, 1 \mathrm{H}), 6.77(\mathrm{~m}, 1 \mathrm{H}), 7.09-7.43(\mathrm{~m}, 7 \mathrm{H}), 7.61-7.79(\mathrm{~m}, 4 \mathrm{H}) .{ }^{13} \mathbf{C} \mathbf{N M R}\left(150.9 \mathrm{MHz}, \mathrm{CDCl}_{3}\right) \delta 31.3$, $31.3,34.4,34.5,59.0,59.1,64.1,65.0,68.6,68.9,70.0,70.0,70.7,70.7,72.0(\times 2 \mathrm{C}), 77.0,77.8$, $111.8,112.1,112.1,112.6,116.8,116.8,121.8,122.0,125.0,125.0,125.4,125.6,125.6,125.8,125.8$, $125.9,126.3,126.3,126.4,127.0,127.5,127.6,127.7,127.9,127.9,127.9,132.8,132.9,133.1,133.3$, $136.9,137.4,137.7,137.9,138.2,138.2,146.2,146.4,150.6,150.7$. HRMS-DART $(\mathrm{m} / \mathrm{z}):[\mathrm{M}+\mathrm{H}]^{+}$ calcd for $\mathrm{C}_{33} \mathrm{H}_{40} \mathrm{~N}_{1} \mathrm{O}_{4}, 514.2952$; found, 514.2945. $[\alpha]_{\mathrm{D}}{ }^{25}+5.6$ (c $\left.0.72, \mathrm{CHCl}_{3}\right)$. The ee value $(88 \%$ ee/89\% ee) was determined by chiral HPLC analysis [CHIRALCEL ${ }^{\circledR}$ AD-3+AD-H column, $4.6 \mathrm{~mm}$ $\times 250 \mathrm{~mm}$, Daicel Chemical Industries, hexane/2-propanol $=95: 5,0.5 \mathrm{~mL} / \mathrm{min}, 40{ }^{\circ} \mathrm{C}, 250 \mathrm{~nm} \mathrm{UV}$ detector, $t_{\mathrm{R}}=128.0 \min \left(\right.$ major $\left._{\mathrm{B}}\right), 156.0 \min \left(\operatorname{minor}_{\mathrm{A}}\right), 163.6 \min \left(\right.$ major $\left._{\mathrm{A}}\right), 170.3 \min \left(\right.$ minor $\left.\left._{\mathrm{B}}\right)\right]$. The absolute configuration of $\mathbf{3 c k}$ was assigned by consideration of the stereochemical pathway.

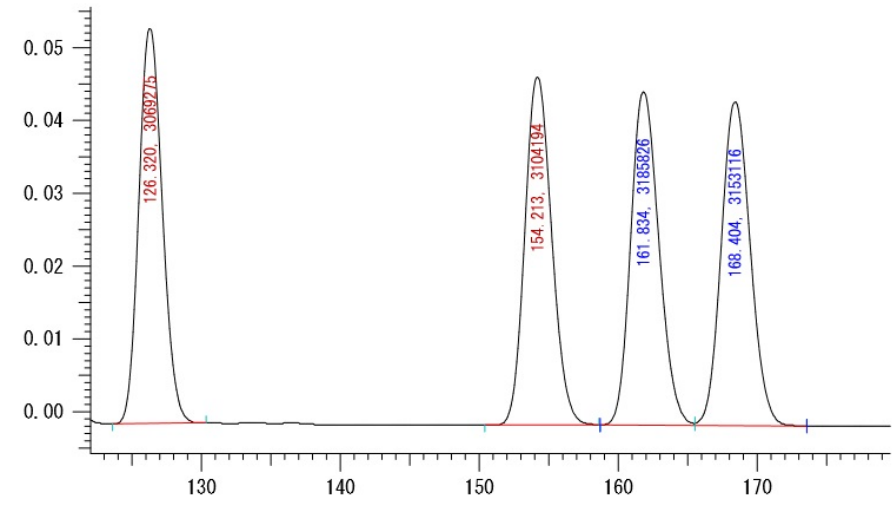

\section{rac-3ck}

$\begin{array}{llll}\text { No } & \text { rt (min) } & \text { area } & \text { area }(\%) \\ 1 & 126.32 & 3069275 & 24.530 \\ 2 & 154.21 & 3104194 & 24.809 \\ 3 & 161.83 & 3185826 & 25.461 \\ 4 & 168.40 & 3153116 & 25.200\end{array}$

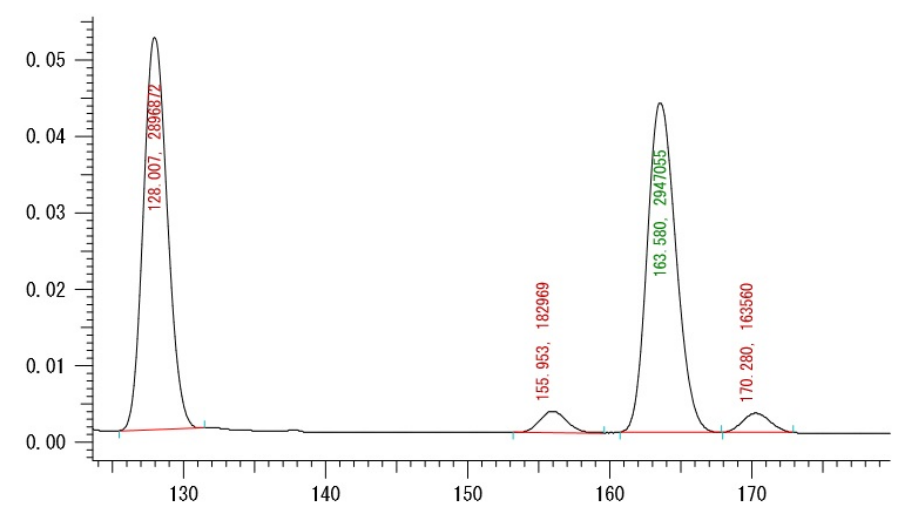

(1S)-3ck

$\begin{array}{llll}\text { No } & \text { rt (min) } & \text { area } & \text { area (\%) } \\ 1 & 128.01 & 2896872 & 46.789 \\ 2 & 155.95 & 182969 & 2.955 \\ 3 & 163.58 & 2947055 & 47.600 \\ 4 & 170.28 & 163560 & 2.642\end{array}$




\section{yl)ethan-1-ol (3cl)}

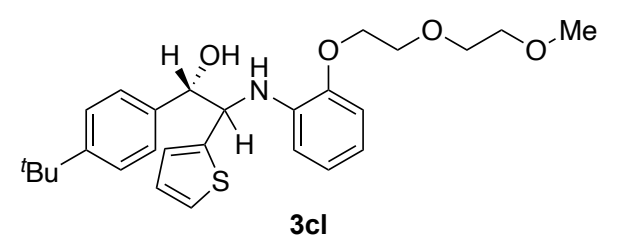

The product 3cl was purified by flash chromatography on silica gel (hexane/Et 3 N/EtOAc 5:0.5:9530:0.5:70) (Scheme 1; $73.7 \mathrm{mg}, 0.157 \mathrm{mmol}, 78 \%$ isolated yield). The diastereomeric ratio (1:1) was determined by ${ }^{1} \mathrm{H}$ NMR. Yellow oil. IR (neat) 737, 1109, 1216, 1444, 1510, 1601, 2872, 2901, 2961, $3064,3394 \mathrm{~cm}^{-1} .{ }^{1} \mathbf{H}$ NMR $\left(600 \mathrm{MHz}, \mathrm{CDCl}_{3}\right) \delta 1.29(\mathrm{~s}, 0.5 \times 9 \mathrm{H}), 1.30(\mathrm{~s}, 0.5 \times 9 \mathrm{H}), 2.93(\mathrm{~m}, 0.5$ $\times 1 \mathrm{H}), 3.25(\mathrm{brs}, 0.5 \times 1 \mathrm{H}), 3.37(\mathrm{~s}, 0.5 \times 3 \mathrm{H}), 3.38(\mathrm{~s}, 0.5 \times 3 \mathrm{H}), 3.58-3.61(\mathrm{~m}, 2 \mathrm{H}), 3.71-3.91(\mathrm{~m}$, $4 \mathrm{H}), 4.06-4.19(\mathrm{~m}, 2 \mathrm{H}), 4.76(\mathrm{~d}, J=4.2 \mathrm{~Hz}, 0.5 \times 1 \mathrm{H}), 4.86(\mathrm{brs}, 0.5 \times 1 \mathrm{H}), 4.90(\mathrm{dd}, J=6.0,3.0$ $\mathrm{Hz}, 0.5 \times 1 \mathrm{H}), 5.04(\mathrm{t}, J=4.8 \mathrm{~Hz}, 0.5 \times 1 \mathrm{H}), 5.16($ brs, $0.5 \times 1 \mathrm{H}), 5.36($ brs, $0.5 \times 1 \mathrm{H}), 6.53(\mathrm{~m}, 1 \mathrm{H})$, $6.62(\mathrm{~m}, 1 \mathrm{H}), 6.71-6.80(\mathrm{~m}, 3 \mathrm{H}), 6.86(\mathrm{~m}, 1 \mathrm{H}), 7.12-7.33(\mathrm{~m}, 5 \mathrm{H}) .{ }^{13} \mathbf{C} \mathbf{N M R}\left(150.9 \mathrm{MHz}, \mathrm{CDCl}_{3}\right)$ $\delta 31.3(\times 2 \mathrm{C}), 34.5(\times 2 \mathrm{C}), 59.0,59.0,60.4,60.9,68.6,68.9,69.8,69.9,70.6,70.7,72.0(\times 2 \mathrm{C}), 76.4$, $77.9,111.9,112.1,112.4,112.8,117.3,117.4,121.9,122.0,124.2,124.7,124.8,125.0,125.1,125.6$, $126.1,126.4,126.5,126.6,137.3,137.4,137.6,138.0,143.6,145.3,146.3,146.5,150.7,150.8$. HRMS-DART $(\mathrm{m} / \mathrm{z})$ : $[\mathrm{M}+\mathrm{H}]^{+}$calcd for $\mathrm{C}_{27} \mathrm{H}_{36} \mathrm{~N}_{1} \mathrm{O}_{4} \mathrm{~S}_{1}, 470.2360$; found, 470.2361. [ $\left.\alpha\right]_{\mathrm{D}}{ }^{25}+10.8(\mathrm{c}$ $\left.0.74, \mathrm{CHCl}_{3}\right)$. The ee value $(85 \%$ ee/ $86 \%$ ee) was determined by chiral HPLC analysis [CHIRALCEL ${ }^{\circledR}$ AD-3+AD-H column, $4.6 \mathrm{~mm} \times 250 \mathrm{~mm}$, Daicel Chemical Industries, hexane/2propanol $=93: 7,0.5 \mathrm{~mL} / \mathrm{min}, 40{ }^{\circ} \mathrm{C}, 250 \mathrm{~nm} \mathrm{UV}$ detector, $\mathrm{t}_{\mathrm{R}}=83.0 \mathrm{~min}\left(\right.$ major $\left._{\mathrm{A}}\right), 86.6 \mathrm{~min}\left(\right.$ minor $\left._{\mathrm{B}}\right)$, $104.0 \mathrm{~min}\left(\right.$ major $\left._{\mathrm{B}}\right), 126.3 \mathrm{~min}\left(\right.$ minor $\left.\left._{\mathrm{A}}\right)\right]$. The absolute configuration of $\mathbf{3 c l}$ was assigned by consideration of the stereochemical pathway.

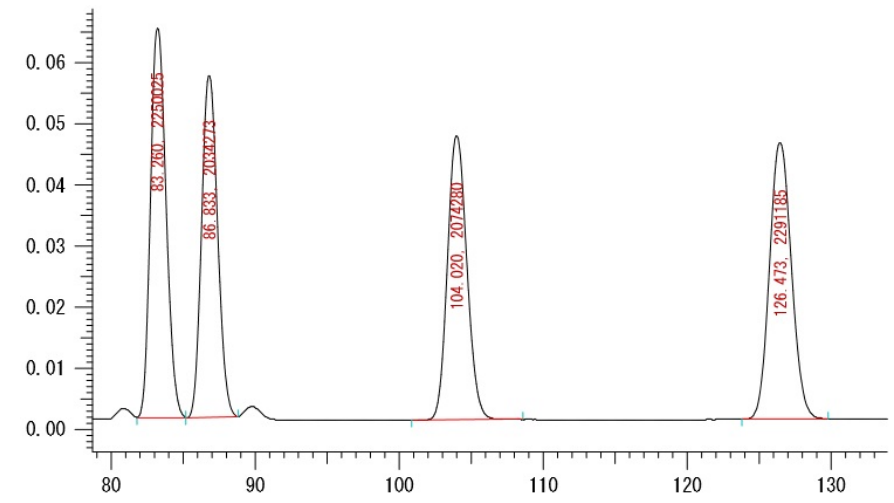

rac-3cl

$\begin{array}{llll}\text { No } & \text { rt (min) } & \text { area } & \text { area (\%) } \\ 1 & 83.26 & 2250025 & 26.013 \\ 2 & 86.83 & 2034273 & 23.518 \\ 3 & 104.02 & 2074280 & 23.981 \\ 4 & 126.47 & 2291185 & 26.488\end{array}$

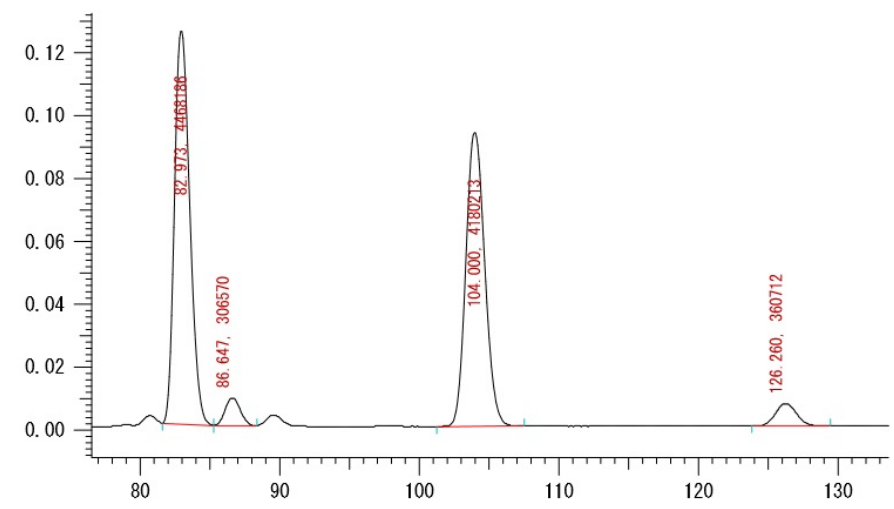

$(1 S)-\mathbf{3 c l}$

$\begin{array}{llll}\text { No } & \text { rt }(\mathrm{min}) & \text { area } & \text { area }(\%) \\ 1 & 82.97 & 4468186 & 47.946 \\ 2 & 86.65 & 306570 & 3.290 \\ 3 & 104.00 & 4180213 & 44.856 \\ 4 & 126.26 & 360712 & 3.871\end{array}$




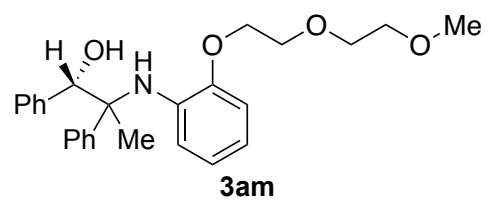

The product 3am was purified by flash chromatography on silica gel (hexane/Et 3 N/EtOAc 5:0.5:95-30:0.5:70) (Scheme 1; $33.1 \mathrm{mg}, 0.079 \mathrm{mmol}, 39 \%$ isolated yield). The diastereomeric ratio (1:1.33) was determined by ${ }^{1} \mathrm{H}$ NMR. Yellow oil. IR (neat) 702, 1046, 1108, 1224, 1448, 1510, 1600, 2825, 2878, 2927, 2982, 3029, 3059, $3387 \mathrm{~cm}^{-1} .{ }^{1} \mathbf{H} \mathbf{N M R}\left(600 \mathrm{MHz}, \mathrm{CDCl}_{3}\right) \delta 1.58(\mathrm{~s}, 0.43 \times 3 \mathrm{H})$, $1.71(\mathrm{~s}, 0.57 \times 3 \mathrm{H}), 2.84(\mathrm{brs}, 0.57 \times 1 \mathrm{H}), 3.31(\mathrm{~s}, 0.43 \times 3 \mathrm{H}), 3.35(\mathrm{~s}, 0.57 \times 3 \mathrm{H}), 3.60-3.65(\mathrm{~m}, 2 \mathrm{H})$, 3.68 (brs, $0.43 \times 1 \mathrm{H}), 3.76-3.96(\mathrm{~m}, 4 \mathrm{H}), 4.10-4.21(\mathrm{~m}, 2 \mathrm{H}), 4.78(\mathrm{~s}, 0.57 \times 1 \mathrm{H}), 4.81(\mathrm{~s}, 0.43 \times 1 \mathrm{H})$, 5.35 (brs, $0.43 \times 1 \mathrm{H}), 5.99(\mathrm{~m}, 1 \mathrm{H}), 6.50-6.54(\mathrm{~m}, 2 \mathrm{H}), 6.76-6.87(\mathrm{~m}, 2 \mathrm{H}), 7.12-7.35(\mathrm{~m}, 9 \mathrm{H}) .{ }^{13} \mathrm{C}$ NMR $\left(100.5 \mathrm{MHz}, \mathrm{CDCl}_{3}\right) \delta 15.7,19.7,59.0,59.0,62.3,62.5,68.5,69.7,70.0,70.3,70.7(\times 2 \mathrm{C})$, 72.0, 72.2, 77.2, 82.1, 82.4, 112.4, 114.1, 114.3, 114.5, 116.1, 116.5, 121.3, 121.8, 126.9, 126.9, 127.1, $127.2,127.5,127.6,127.8,127.8,127.9,128.1,128.2(\times 2 \mathrm{C}), 136.2,137.4,139.3,139.7,142.3,143.5$, $147.1(\times 2 \mathrm{C})$. HRMS-DART $(\mathrm{m} / \mathrm{z})$ : $[\mathrm{M}+\mathrm{H}]^{+}$calcd for $\mathrm{C}_{26} \mathrm{H}_{32} \mathrm{~N}_{1} \mathrm{O}_{4}, 422.2326$; found, 422.2321. $[\alpha]_{\mathrm{D}}{ }^{26}-6.9\left(c 0.18, \mathrm{CHCl}_{3}\right)$. The ee value $(88 \%$ ee/ $87 \%$ ee $)$ was determined by chiral HPLC analysis [CHIRALCEL ${ }^{\circledR}$ AD-3 column, $4.6 \mathrm{~mm} \times 250 \mathrm{~mm}$, Daicel Chemical Industries, hexane/2-propanol = 93:7, $0.5 \mathrm{~mL} / \mathrm{min}, 40{ }^{\circ} \mathrm{C}, 250 \mathrm{~nm} \mathrm{UV}$ detector, $\mathrm{t}_{\mathrm{R}}=19.1 \mathrm{~min}\left(\right.$ minor $\left._{\mathrm{B}}\right), 21.1 \mathrm{~min}$ (major $), 29.2 \mathrm{~min}$ $\left(\operatorname{minor}_{\mathrm{A}}\right), 32.5 \mathrm{~min}\left(\right.$ major $\left._{\mathrm{A}}\right)$,]. The absolute configuration of 3am was assigned by consideration of the stereochemical pathway.

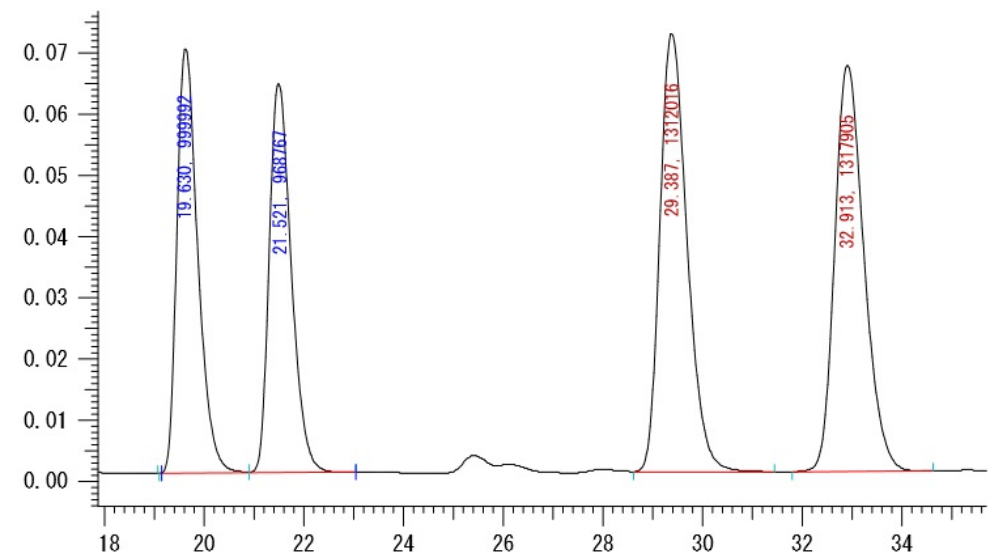

\section{rac-3am}

No $\quad \mathrm{rt}(\mathrm{min})$ area $\quad$ area $(\%)$

$1 \quad 19.63 \quad 999992 \quad 21.745$

$2 \quad 21.52 \quad 968767 \quad 21.066$

$3 \quad 29.39 \quad 1312016 \quad 28.530$

$4 \quad 32.91 \quad 1317905 \quad 28.658$

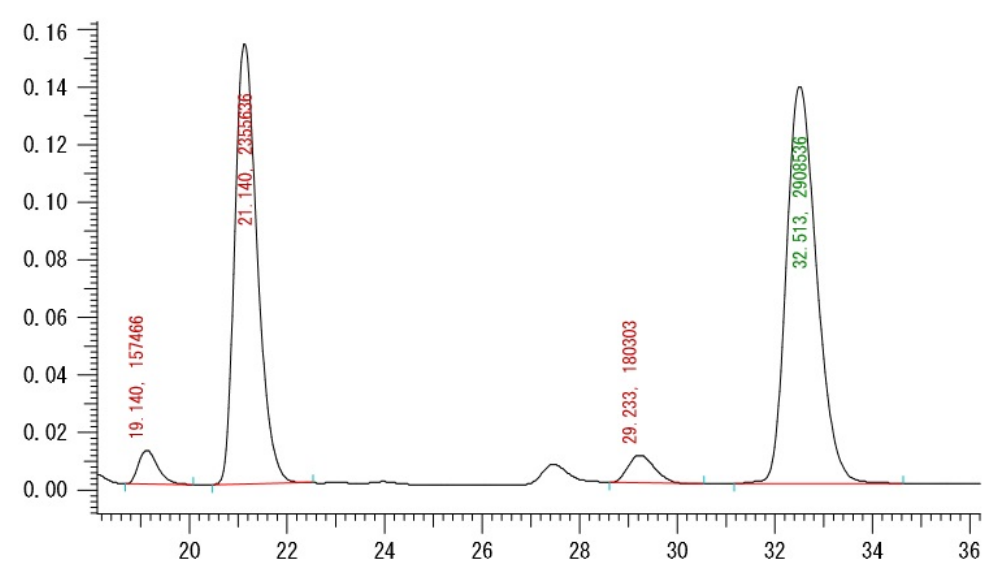

(1S)-3am

$\begin{array}{llll}\text { No } & \text { rt (min) } & \text { area } & \text { area (\%) } \\ 1 & 19.14 & 157466 & 2.811 \\ 2 & 21.14 & 2355636 & 42.050 \\ 3 & 29.23 & 180303 & 3.219 \\ 4 & 32.51 & 2908536 & 51.920\end{array}$




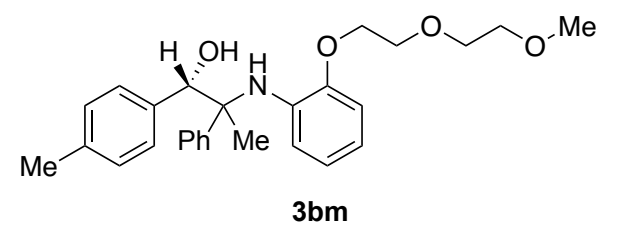

The product $3 \mathbf{b m}$ was purified by flash chromatography on silica gel (hexane/Et $\mathrm{t}_{3} \mathrm{~N} / \mathrm{EtOAc}$ 5:0.5:95-30:0.5:70) (Scheme 1; $30.7 \mathrm{mg}, 0.070 \mathrm{mmol}, 35 \%$ isolated yield). The diastereomeric ratio (1:1.50) was determined by ${ }^{1} \mathrm{H}$ NMR. Yellow oil. IR (neat) 739, 1048, 1112, 1224, 1447, 1510, 1600, 2876, 2922, 2982, 3023, 3055, $3388 \mathrm{~cm}^{-1} .{ }^{1} \mathbf{H}$ NMR (400 MHz, $\left.\mathrm{CDCl}_{3}\right) \delta 1.57(\mathrm{~s}, 0.40 \times 3 \mathrm{H}), 1.70$ $(\mathrm{s}, 0.60 \times 3 \mathrm{H}), 2.28(\mathrm{~s}, 0.40 \times 3 \mathrm{H}), 2.32(\mathrm{~s}, 0.60 \times 3 \mathrm{H}), 2.72(\mathrm{brs}, 0.60 \times 1 \mathrm{H}), 3.32(\mathrm{~s}, 0.40 \times 3 \mathrm{H})$, $3.36(\mathrm{~s}, 0.60 \times 3 \mathrm{H}), 3.53(\mathrm{brs}, 0.40 \times 1 \mathrm{H}), 3.60-3.65(\mathrm{~m}, 2 \mathrm{H}), 3.76-3.98(\mathrm{~m}, 4 \mathrm{H}), 4.09-4.21(\mathrm{~m}, 2 \mathrm{H})$, $4.75(\mathrm{~s}, 0.60 \times 1 \mathrm{H}), 4.78(\mathrm{~s}, 0.40 \times 1 \mathrm{H}), 5.30(\mathrm{brs}, 0.40 \times 1 \mathrm{H}), 5.97(\mathrm{~m}, 1 \mathrm{H}), 6.49-6.54(\mathrm{~m}, 2 \mathrm{H}), 6.75-$ $6.83(\mathrm{~m}, 2 \mathrm{H}), 6.93-7.06(\mathrm{~m}, 3 \mathrm{H}), 7.12-7.37$ (m, 5H). ${ }^{13} \mathbf{C} \mathbf{N M R}\left(150.9 \mathrm{MHz}, \mathrm{CDCl}_{3}\right) \delta 15.9,19.6$, 21.1, 21.11, 59.0, 59.0, 62.2, 62.5, 68.4, 69.4, 70.0, 70.3, 70.7 ( × 2C), 72.0, 72.1, 82.0, 82.3, 112.3, $113.7,114.2,114.4,116.0,116.4,121.2,121.7,126.8,126.9,127.7,127.7,127.8,127.8,128.1,128.1$, $128.1,128.3,136.2,136.3,136.7,136.8,137.3,137.4,142.5,143.6,147.0,147.1$. HRMS-DART $(m / z):[\mathrm{M}+\mathrm{H}]^{+}$calcd for $\mathrm{C}_{27} \mathrm{H}_{34} \mathrm{~N}_{1} \mathrm{O}_{4}, 436.2482$; found, 436.2487. [ $\left.\alpha\right]_{\mathrm{D}}{ }^{26}-7.2\left(c 0.15, \mathrm{CHCl}_{3}\right)$. The ee value $\left(86 \%\right.$ ee/ $86 \%$ ee) was determined by chiral HPLC analysis [CHIRALCEL ${ }^{\circledR} \mathrm{AD}-3$ column, $4.6 \mathrm{~mm} \times 250 \mathrm{~mm}$, Daicel Chemical Industries, hexane/2-propanol =95:5, $0.5 \mathrm{~mL} / \mathrm{min}, 40{ }^{\circ} \mathrm{C}, 250$ $\mathrm{nm}$ UV detector, $\mathrm{t}_{\mathrm{R}} 25.7 \min \left(\right.$ major $\left._{\mathrm{B}}\right), 27.7 \min \left(\operatorname{minor}_{\mathrm{B}}\right), 44.5 \min \left(\operatorname{minor}_{\mathrm{A}}\right), 48.8 \min \left(\right.$ major $\left.\left._{\mathrm{A}}\right)\right]$. The absolute configuration of $\mathbf{3} \mathbf{b m}$ was assigned by consideration of the stereochemical pathway.

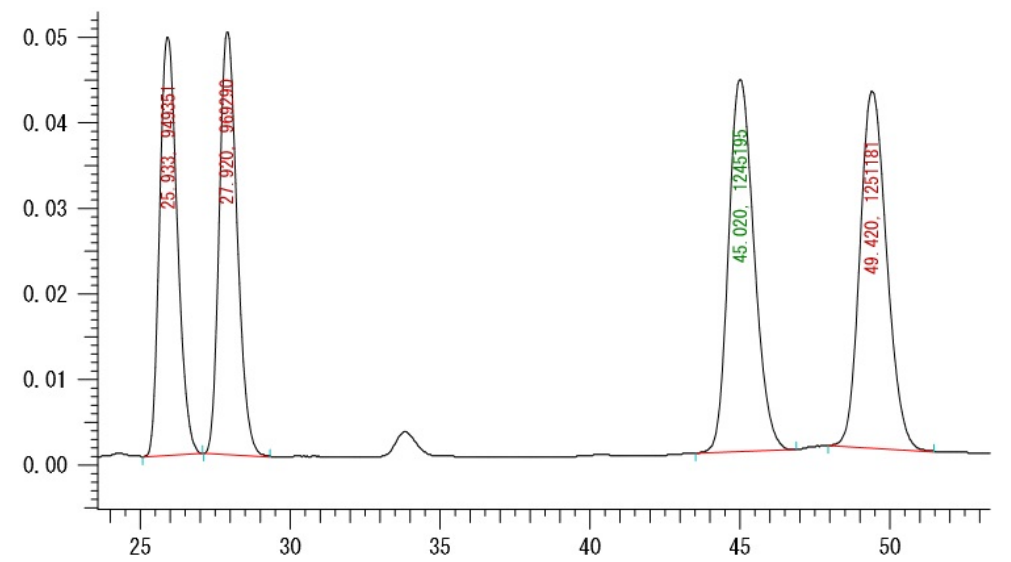

rac-3bm

No $\mathrm{rt}(\mathrm{min})$ area $\quad$ area $(\%)$

$1 \quad 25.93 \quad 949351 \quad 21.503$

$\begin{array}{llll}2 & 27.92 & 969290 & 21.954\end{array}$

$\begin{array}{llll}3 & 45.02 & 1245195 & 28.204\end{array}$

$\begin{array}{llll}4 & 49.42 & 1251181 & 28.339\end{array}$

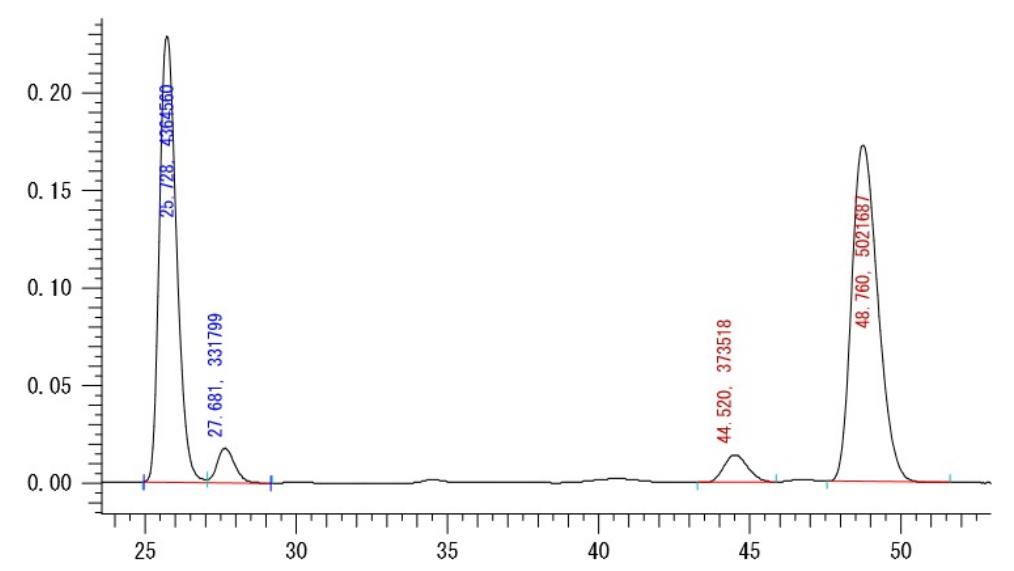

(1S)-3bm

$\begin{array}{llll}\text { No } & \text { rt (min) } & \text { area } & \text { area }(\%) \\ 1 & 25.73 & 4364560 & 43.250 \\ 2 & 27.68 & 331799 & 3.288 \\ 3 & 44.52 & 373518 & 3.701 \\ 4 & 48.76 & 5021687 & 49.761\end{array}$




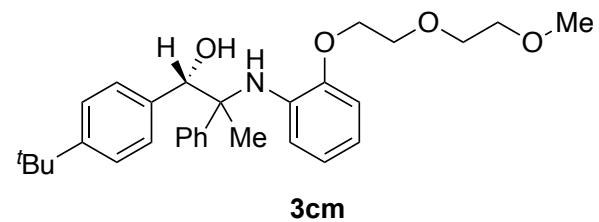

The product $3 \mathbf{c m}$ was purified by flash chromatography on silica gel (hexane/Et ${ }_{3} \mathrm{~N} / \mathrm{EtOAc}$ 5:0.5:95-30:0.5:70) (Scheme 1; $34.9 \mathrm{mg}, 0.073 \mathrm{mmol}, 37 \%$ isolated yield). The diastereomeric ratio (1:1.08) was determined by ${ }^{1} \mathrm{H}$ NMR. Yellow oil. IR (neat) 701, 1047, 1109, 1223, 1447, 1509, 1600, 2872, 2903, 2960, 3030, 3057, $3390 \mathrm{~cm}^{-1} .{ }^{1} \mathbf{H}$ NMR (600 MHz, $\left.\mathrm{CDCl}_{3}\right) \delta 1.27(\mathrm{~s}, 0.48 \times 9 \mathrm{H}), 1.30$ $(\mathrm{s}, 0.52 \times 9 \mathrm{H}), 1.58(\mathrm{~s}, 0.48 \times 3 \mathrm{H}), 1.71(\mathrm{~s}, 0.52 \times 3 \mathrm{H}), 2.60(\mathrm{brs}, 0.48 \times 1 \mathrm{H}), 3.32(\mathrm{~m}, 0.52 \times 1 \mathrm{H})$, $3.34(\mathrm{~s}, 0.48 \times 3 \mathrm{H}), 3.36(\mathrm{~s}, 0.52 \times 3 \mathrm{H}), 3.60-3.63(\mathrm{~m}, 2 \mathrm{H}), 3.77-3.97(\mathrm{~m}, 4 \mathrm{H}), 4.08-4.20(\mathrm{~m}, 2 \mathrm{H})$, $4.73(\mathrm{~s}, 0.52 \times 1 \mathrm{H}), 4.77(\mathrm{~s}, 0.48 \times 1 \mathrm{H}), 5.30$ (brs, $0.48 \times 1 \mathrm{H}), 5.93$ (brs, $0.52 \times 1 \mathrm{H}), 5.97(\mathrm{~m}, 1 \mathrm{H})$, 6.48-6.52 (m, 2H), 6.74-6.85 (m, 2H), 7.09-7.17 (m, 2H), 7.24-7.31 (m, 5H), $7.38(\mathrm{~m}, 1 \mathrm{H}) .{ }^{13} \mathbf{C}$ NMR $\left(150.9 \mathrm{MHz}, \mathrm{CDCl}_{3}\right) \delta 16.5,19.3,31.3(\times 2 \mathrm{C}), 34.4,34.5,59.0(\times 2 \mathrm{C}), 62.2,62.5,68.3,69.2$, 70.0, 70.2, 70.7 ( $\times 2 \mathrm{C}), 72.0,72.1,82.1,82.2,112.1,113.3,114.2,114.3,116.0,116.3,121.1,121.5$, 124.0, 124.5, 126.8, 126.9, 127.5, 127.7, 127.7, 127.8, $128.1(\times 2 \mathrm{C}), 136.1,136.2,136.5,137.1,142.7$, 143.5, 147.0, 147.1, 150.2, 150.8. HRMS-DART $(\mathrm{m} / \mathrm{z})$ : $[\mathrm{M}+\mathrm{H}]^{+}$calcd for $\mathrm{C}_{30} \mathrm{H}_{40} \mathrm{~N}_{1} \mathrm{O}_{4}, 478.2952$; found, $478.2955 .[\alpha]_{\mathrm{D}}{ }^{30}-3.2^{\circ}\left(c 0.79, \mathrm{CHCl}_{3}\right)$. The ee value $(84 \%$ ee $/ 84 \%$ ee $)$ was determined by chiral HPLC analysis [CHIRALCEL ${ }^{\circledR}$ AD-3 column, $4.6 \mathrm{~mm} \times 250 \mathrm{~mm}$, Daicel Chemical Industries, hexane/2-propanol $=95: 5,0.5 \mathrm{~mL} / \mathrm{min}, 40{ }^{\circ} \mathrm{C}, 250 \mathrm{~nm} \mathrm{UV}$ detector, $\mathrm{t}_{\mathrm{R}}=18.6 \mathrm{~min}$ ( major $_{\mathrm{B}}$ ), $20.2 \mathrm{~min}$ (minor $\left.\mathrm{B}), 31.8 \mathrm{~min}\left(\operatorname{major}_{\mathrm{A}}\right), 38.2 \mathrm{~min}\left(\operatorname{minor}_{\mathrm{A}}\right)\right]$. The absolute configuration of $\mathbf{3} \mathbf{c m}$ was assigned by consideration of the stereochemical pathway.

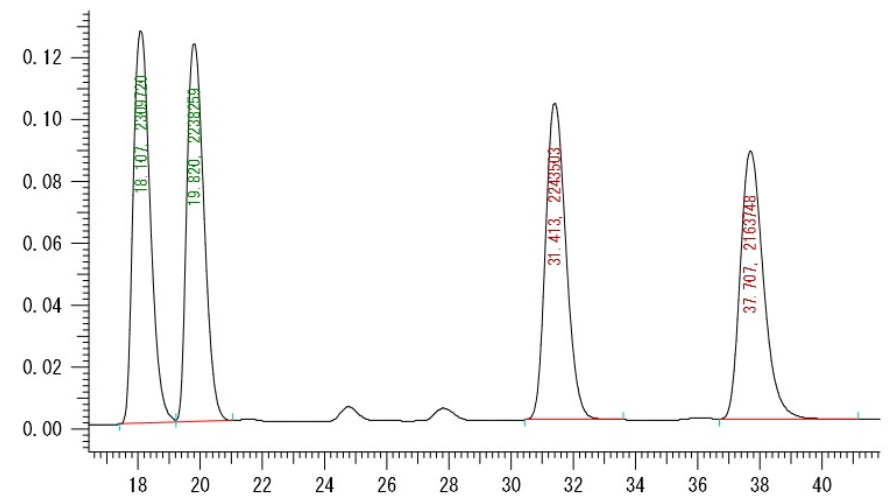

$$
r a c-\mathbf{3 c m}
$$

$\begin{array}{llll}\text { No } & \text { rt }(\mathrm{min}) & \text { area } & \text { area (\%) } \\ 1 & 18.11 & 2309720 & 25.792 \\ 2 & 19.82 & 2238259 & 24.994 \\ 3 & 31.41 & 2243503 & 25.052 \\ 4 & 37.71 & 2163748 & 24.162\end{array}$

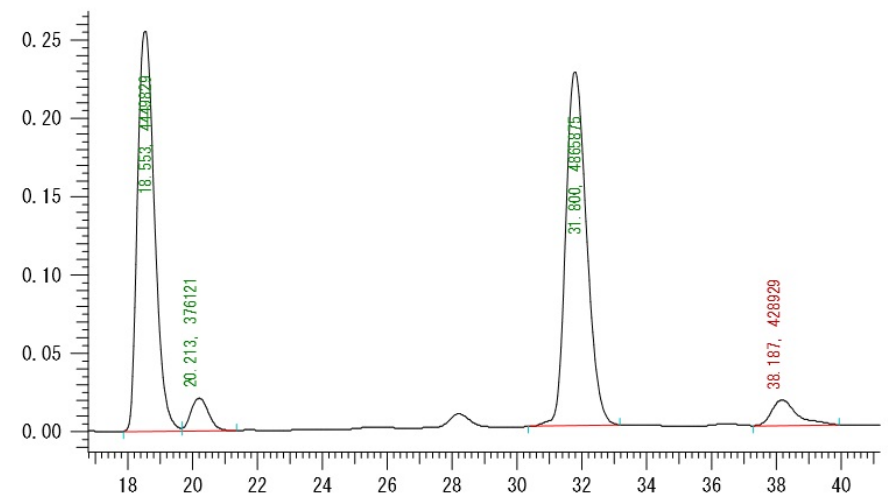

$(1 S)-\mathbf{3 c m}$

$\begin{array}{llll}\text { No } & \text { rt }(\mathrm{min}) & \text { area } & \text { area }(\%) \\ 1 & 18.55 & 4449829 & 43.965 \\ 2 & 20.21 & 376121 & 3.716 \\ 3 & 31.80 & 4865875 & 48.075 \\ 4 & 38.19 & 428929 & 4.238\end{array}$



ol (3gm)

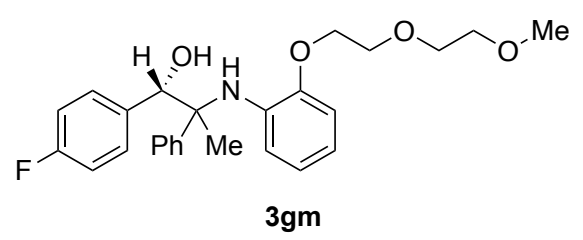

The product 3gm was purified by flash chromatography on silica gel (hexane/Et $t_{3} \mathrm{~N} / \mathrm{EtOAc}$ 5:0.5:95-30:0.5:70) (Scheme 1; $31.7 \mathrm{mg}, 0.072 \mathrm{mmol}, 36 \%$ isolated yield). The diastereomeric ratio (1:1.44) was determined by ${ }^{1} \mathrm{H}$ NMR. Yellow oil. IR (neat) 765, 1106, 1221, 1508, 1601, 2880, 2924 , 2985, 3057, $3389 \mathrm{~cm}^{-1} .{ }^{1} \mathbf{H}$ NMR $\left(600 \mathrm{MHz}, \mathrm{CDCl}_{3}\right) \delta 1.55(\mathrm{~s}, 0.41 \times 3 \mathrm{H}), 1.70(\mathrm{~s}, 0.59 \times 3 \mathrm{H}), 3.09$ (brs, $0.59 \times 1 \mathrm{H}), 3.29(\mathrm{~s}, 0.41 \times 3 \mathrm{H}), 3.33(\mathrm{~s}, 0.59 \times 3 \mathrm{H}), 3.60-3.67(\mathrm{~m}, 2 \mathrm{H}), 3.75-3.82(\mathrm{~m}, 2 \mathrm{H})$, 3.83-3.95 (m, 2H), $3.98($ brs, $0.41 \times 1 \mathrm{H}), 4.11-4.22(\mathrm{~m}, 2 \mathrm{H}), 4.76(\mathrm{~d}, J=1.8 \mathrm{~Hz}, 0.59 \times 1 \mathrm{H}), 4.78(\mathrm{~s}$, $0.41 \times 1 \mathrm{H}), 5.35(\mathrm{brs}, 0.41 \times 1 \mathrm{H}), 5.98(\mathrm{~m}, 1 \mathrm{H}), 6.51-6.56(\mathrm{~m}, 2 \mathrm{H}), 6.78-6.92(\mathrm{~m}, 4 \mathrm{H}), 7.04(\mathrm{~m}, 1 \mathrm{H})$, 7.24-7.30 (m, 5H). ${ }^{13} \mathbf{C}$ NMR (150.9 MHz, $\left.\mathrm{CDCl}_{3}\right) \delta 15.3,20.1,59.0(\times 2 \mathrm{C}), 62.3,62.5,68.8,70.0$, 70.1, 70.4, 70.6, 70.6, 72.0, 72.2, 81.4, 81.8, 112.8, 113.8 (d, $J=21.7 \mathrm{~Hz}), 114.3$ (d, $J=21.0 \mathrm{~Hz})$, $114.3,114.6,114.7,116.2,116.7,121.4,122.1,126.9,127.0,127.6,127.8,128.1,128.2,129.3(\mathrm{~d}, J$ $=7.9 \mathrm{~Hz}), 129.7(\mathrm{~d}, J=7.9 \mathrm{~Hz}), 135.1(\mathrm{~d}, J=2.9 \mathrm{~Hz}), 135.6(\mathrm{~d}, J=2.9 \mathrm{~Hz}), 136.3,137.6,141.9$, 143.4, 147.1, 147.2, $162.0(\mathrm{~d}, J=244.6 \mathrm{~Hz}), 162.2(\mathrm{~d}, J=246.3 \mathrm{~Hz})$. HRMS-DART $(\mathrm{m} / \mathrm{z}):[\mathrm{M}+\mathrm{H}]^{+}$ calcd for $\mathrm{C}_{26} \mathrm{H}_{31} \mathrm{~F}_{1} \mathrm{~N}_{1} \mathrm{O}_{4}, 440.2232$; found, 440.2236. $[\alpha]_{\mathrm{D}}{ }^{25}+2.8$ (c $\left.0.22, \mathrm{CHCl}_{3}\right)$. The ee value $(88 \%$ ee/90\% ee) was determined by chiral HPLC analysis [CHIRALCEL ${ }^{\circledR}$ AD-3+AD-H column, $4.6 \mathrm{~mm}$ $\times 250 \mathrm{~mm}$, Daicel Chemical Industries, hexane/2-propanol $=95: 5,0.5 \mathrm{~mL} / \mathrm{min}, 40{ }^{\circ} \mathrm{C}, 250 \mathrm{~nm} \mathrm{UV}$ detector, $t_{R}=42.3 \min \left(\operatorname{minor}_{B}\right), 45.7 \min \left(\right.$ major $\left.\left._{B}\right), 67.8 \min \left(\operatorname{minor}_{\mathrm{A}}\right), 71.2 \min \left(\operatorname{major}_{\mathrm{A}}\right)\right]$. The absolute configuration of $\mathbf{3 g m}$ was assigned by consideration of the stereochemical pathway.

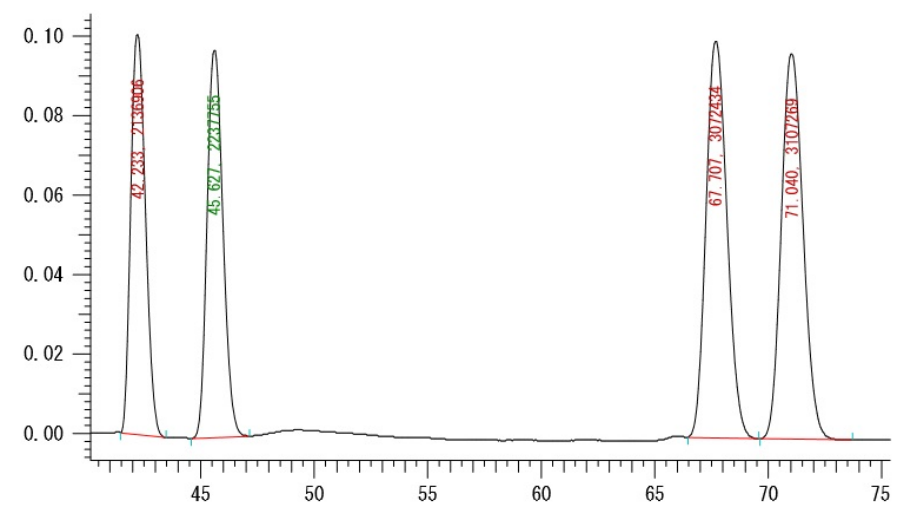

\section{rac-3gm}

$\begin{array}{llll}\text { No } & \text { rt (min) } & \text { area } & \text { area (\%) } \\ 1 & 42.23 & 2136906 & 20.247 \\ 2 & 45.63 & 2237755 & 21.202 \\ 3 & 67.71 & 3072434 & 29.111 \\ 4 & 71.04 & 3107269 & 29.441\end{array}$

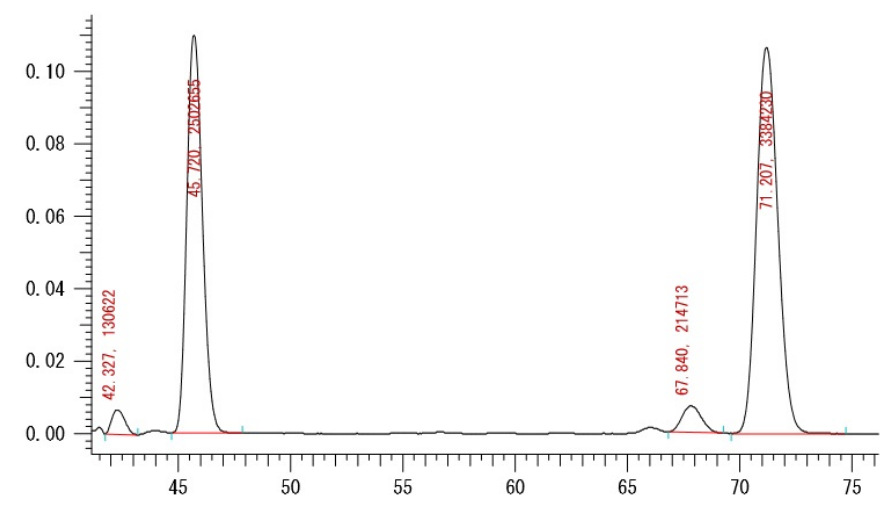

\section{(1S)-3gm}

$\begin{array}{llll}\text { No } & \text { rt (min) } & \text { area } & \text { area }(\%) \\ 1 & 42.33 & 130622 & 2.096 \\ 2 & 45.72 & 2502655 & 40.157 \\ 3 & 67.84 & 214713 & 3.445 \\ 4 & 71.21 & 3384230 & 54.302\end{array}$


- Prcedure for Deprotection and Determination of Absolute Configuration

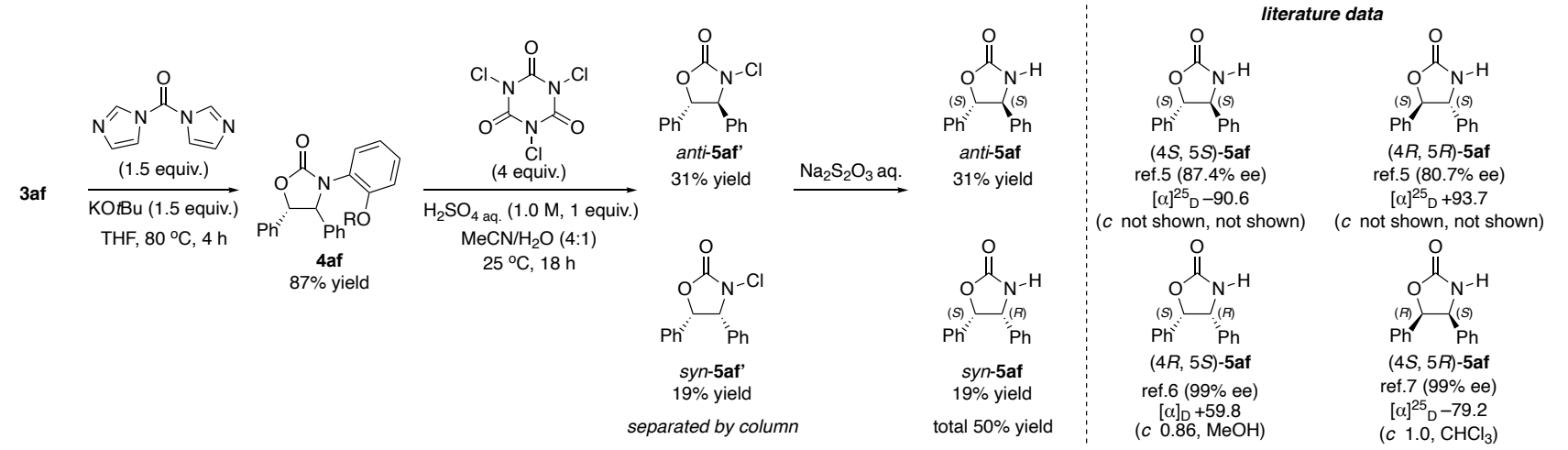

Scheme 2. The chiral $\beta$-aminoalcohol 3af (265 mg, $0.65 \mathrm{mmol}$ ) and 1,1'-carbonyldiimidazole (171 $\mathrm{mg}, 1.05 \mathrm{mmol}$ ) was dissolved in THF $(4.5 \mathrm{ml})$ in a $20 \mathrm{ml}$ vial containing a stir bar. $\mathrm{KOtBu}(118 \mathrm{mg}$, $1.05 \mathrm{mmol}$ ) was added to the reaction solution at room temperature. After stirring at $80{ }^{\circ} \mathrm{C}$ for $4 \mathrm{~h}$, the reaction mixture was treated with $\mathrm{H}_{2} \mathrm{O}$, then extracted with ethyl acetate ( 3 times) and dried over sodium sulfate. After filtration, the resulting solution was evaporated under reduced pressure. The residue was purified by flash chromatography on silica gel (hexane/EtOAc 10:90-0:100) provided $\mathrm{N}$-aryl oxazolidinone 4af (247 mg, $0.57 \mathrm{mmol}$, 87\% isolated yield). The diastereomeric ratio (1:1.22) was determined by ${ }^{1} \mathrm{H}$ NMR. Yellow oil. IR (neat) 699, 752, 1107, 1193, 1383, 1503, 1752, 2823, 2877, 2926, 3035, $3065 \mathrm{~cm}^{-1} .{ }^{1} \mathbf{H}$ NMR $\left(600 \mathrm{MHz}, \mathrm{CDCl}_{3}\right) \delta 3.29(\mathrm{~s}, 0.55 \times 3 \mathrm{H}), 3.42(\mathrm{~s}, 0.45 \times 3 \mathrm{H})$, 3.25-3.57 (m, 2H), $3.66(\mathrm{~m}, 1 \mathrm{H}), 3.77-3.84(\mathrm{~m}, 2 \mathrm{H}), 3.95(\mathrm{~m}, 1 \mathrm{H}), 4.12-4.22(\mathrm{~m}, 2 \mathrm{H}), 5.43(\mathrm{dd}, J=$ 15.0, 7.2 Hz, $0.55 \times 2 \mathrm{H}), 5.86(\mathrm{~d}, J=8.6 \mathrm{~Hz}, 0.45 \times 1 \mathrm{H}), 6.08(\mathrm{~d}, J=8.6 \mathrm{~Hz}, 0.45 \times 1 \mathrm{H}), 6.82-6.89$ (m, 2H), 6.94-7.02 (m, 2H), 7.06-7.19 (m, 4H), 7.26-7.34 (m, 3H), 7.37-7.40 (m, 3H). ${ }^{13}$ C NMR $\left(150.9 \mathrm{MHz}, \mathrm{CDCl}_{3}\right) \delta 58.8,58.9,66.0,67.4,67.7,69.1,69.4,69.6,70.3,70.6,71.6,71.9,79.9,83.7$, $112.4,112.9,120.8,120.9,124.6,125.3,127.6(\times 2 \mathrm{C}), 127.6,127.7(\times 2 \mathrm{C}), 127.7,128.0,128.5,128.6$, $128.7,128.7,128.7,129.1,129.8,134.8,134.9,137.4,138.0,153.8,154.0,156.7,157.2$. HRMSDART $(m / z):[\mathrm{M}+\mathrm{H}]^{+}$calcd for $\mathrm{C}_{26} \mathrm{H}_{28} \mathrm{~N}_{1} \mathrm{O}_{5}, 434.1962$; found, 434.1962. [ $\left.\alpha\right]_{\mathrm{D}}{ }^{25}-53.8(c$ 2.16, $\left.\mathrm{CHCl}_{3}\right)$. To the solution of 4 af $(247 \mathrm{mg}, 0.569 \mathrm{mmol})$ in $\mathrm{MeCN}: \mathrm{H}_{2} \mathrm{O}=4: 1(15 \mathrm{~mL})$ trichloroisocyanuric acid (529 mg, $2.27 \mathrm{mmol}$ ) and $1.0 \mathrm{M} \mathrm{H}_{2} \mathrm{SO}_{4}$ aq. (600 $\left.\mu \mathrm{L}\right)$ were sequentially added. After $18 \mathrm{~h}$ stirring at room temperature, the reaction mixture was treated with $\mathrm{H}_{2} \mathrm{O}$, then extracted with diethyl ether (3 times) and dried over sodium sulfate. After filtration, the resulting solution was evaporated under reduced pressure. The purification by flash chromatography on silica gel (hexane/EtOAc 2:98-15:85) gave anti-5af' (49.3 mg, 0.18 mmol, 31\% yield) and syn-5af' (30.1 $\mathrm{mg}, 0.11 \mathrm{mmol}, 19 \%$ yield). Next, each isomer was separately added to ethyl acetate in a vial and treated with $\mathrm{Na}_{2} \mathrm{~S}_{3} \mathrm{O}_{3}$ aq. After shaking it, the mixture was extracted with ethyl acetate ( 3 times) and dried over sodium sulfate. After filtration, the resulting solution was evaporated under reduced pressure provided the oxazolidinone anti-5af $(42.5 \mathrm{mg}, 0.18 \mathrm{mmol}, 31 \%$ yield $)$ and $s y n-5 \mathrm{af}(25.9 \mathrm{mg}$, $0.11 \mathrm{mmol}, 19 \%$ yield), respectively. The absolute configuration of each isomers was determined by the comparison with optical rotation of the literature data. lit. data. $(4 S, 5 S)$ isomer $\left(87.4 \%\right.$ ee): $[\alpha]_{\mathrm{D}}{ }^{25}$ -90.6 ( $c$ not shown, not shown $)^{5} ;(4 R, 5 R)$ isomer $\left(80.7 \%\right.$ ee): $[\alpha]_{\mathrm{D}}{ }^{25}+93.7(c \text { not shown, not shown })^{5}$; $(4 R, 5 S)$ isomer $\left(99 \%\right.$ ee): $[\alpha]_{\mathrm{D}}+59.8(c 0.86, \mathrm{MeOH})^{6} ;(4 R, 5 S)$ isomer $(99 \%$ ee $):[\alpha]_{\mathrm{D}}-79.2(c 1.0$, $\mathrm{MeOH})^{7}(4 S, 5 S)$ isomer. The absolute configuration of anti-5af $\left\{[\alpha]_{\mathrm{D}}^{27}-71.4\left(c 0.92, \mathrm{CHCl}_{3}\right)\right\}$ and syn-5af $\left\{[\alpha]_{\mathrm{D}}{ }^{27}+49.9\left(c 0.75, \mathrm{CHCl}_{3}\right)\right\}$ were determined to be $(4 S, 5 S)$ and $(4 R, 5 S)$, respectively. 


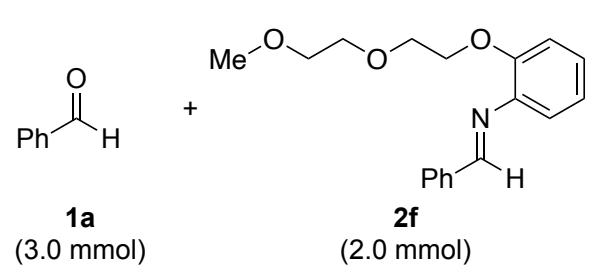

$\mathrm{CuCl}(10 \mathrm{~mol} \%)$

$(S, S)-L_{1} \cdot \mathrm{HBF}_{4}(10 \mathrm{~mol} \%)$

$\mathrm{PhMe}_{2} \mathrm{SiBpin}(1.5$ equiv.)

$\mathrm{NaOtBu}(1.1$ equiv.)

toluene, $80^{\circ} \mathrm{C}, 3 \mathrm{~h}$

then TBAF

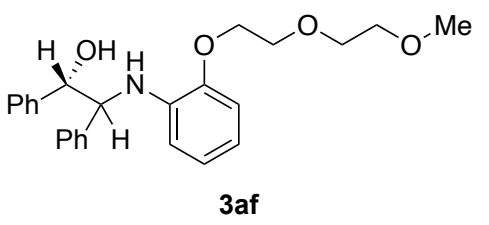

$\mathrm{CuCl}$ (19.8 mg, $0.2 \mathrm{mmol}),(S, S)-\mathbf{L 1} \cdot \mathrm{HBF}_{4}(134.1 \mathrm{mg}, 0.2 \mathrm{mmol})$ and $\mathrm{NaOSiMe}_{3}(246.7 \mathrm{mg}, 2.2$ mmol) were placed in a vial containing a magnetic stirring bar. The vial was sealed with a Teflon ${ }^{\circledR}$ coated silicon rubber septum, and then the vial was evacuated and filled with argon. Cyclooctane (5.0 $\mathrm{mL}$ ) was added to the vial, and then the mixture was stirred at room temperature for $10 \mathrm{~min}$. Next, $\mathrm{PhMe}_{2} \mathrm{SiB}$ (pin) $(818 \mu \mathrm{L}, 3.0 \mathrm{mmol})$ and benzaldehyde (1a) $(304 \mu \mathrm{L}, 3.0 \mathrm{mmol})$ were added, and then the mixture was stirred at room temperature for $20 \mathrm{~min}$. Then, cyclooctane $(5.0 \mathrm{~mL})$ was added to the vial, and $\mathrm{NaOSiMe}_{3}(246.7 \mathrm{mg}, 2.2 \mathrm{mmol})$, imine (2f) $(598.7 \mathrm{mg}, 2.0 \mathrm{mmol})$ was added. After $3 \mathrm{~h}$ stirring at $40{ }^{\circ} \mathrm{C}$ with oil bath, the reaction mixture was treated with TBAF $(6.0 \mathrm{~mL})$ and stirred for 1 h. After addition of water to the reaction, the reaction mixture was extracted with ethyl acetate (3 times), washed with brine and dried over sodium sulfate. After filtration, the resulting solution was evaporated under reduced pressure. purification by flash column chromatography (5:0.5:9530:0.5:70, hexane/Et $\left.{ }_{3} \mathrm{~N} / \mathrm{EtOAc}\right)$ on silica gel gave the $3 \mathrm{af}(659 \mathrm{mg}, 81 \%$, d.r. $51: 49,76 \%$ ee, $76 \%$ ee) as a yellow oil.

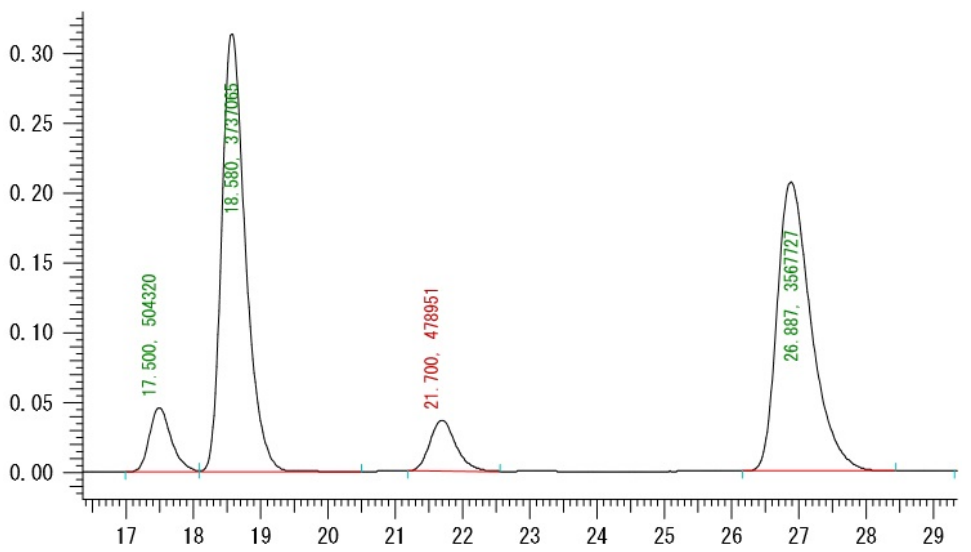

Scale up reaction for $(1 S)$-3af

$\begin{array}{llll}\text { No } & \text { rt (min) } & \text { area } & \text { area }(\%) \\ 1 & 17.50 & 504320 & 6.085 \\ 2 & 18.58 & 3737065 & 45.090 \\ 3 & 21.70 & 478951 & 5.779 \\ 4 & 26.89 & 3567727 & 43.047\end{array}$




\section{- References -}

(1) Morales, S.; Guijarro, F. G.; García Ruano, J. L.; Cid, M. B. A. J. Am. Chem. Soc. 2014, 136, 1082-1089.

(2) Malkov, A. V.; Vranková, K.; Stončius, S.; Kočovský, P. J. Org. Chem. 2009, 74, 5839-5849.

(3) Takeda, M.; Mitsui, A.; Nagao, K.; Ohmiya, H. J. Am. Chem. Soc. 2019, 141, 3664-3669.

(4) Bartoli, G.; Bosco, M.; Carlone, A.; Locatelli, M.; Massaccesi, M.; Melchiorre, P.; Sambri, L. Org. Lett. 2004, 6, 2173-2176.

(5) Barta, N. S.; Sidler, D. R.; Somerville, K. B.; Weissman, S. A.; Larsen, R. D.; Reider, P. J. Org. Lett. 2000, 2, 2821-2824.

(6) Simon, M.; Micle, A.; Badea, V.; Csunderlik, C. Synthetic Communications 2009, 39, 2633.

(7) Qin, Y.; Wang, C.; Huang, Z.; Xiao, X.; Jiang, Y. J. Org. Chem. 2004, 69, 8533. 


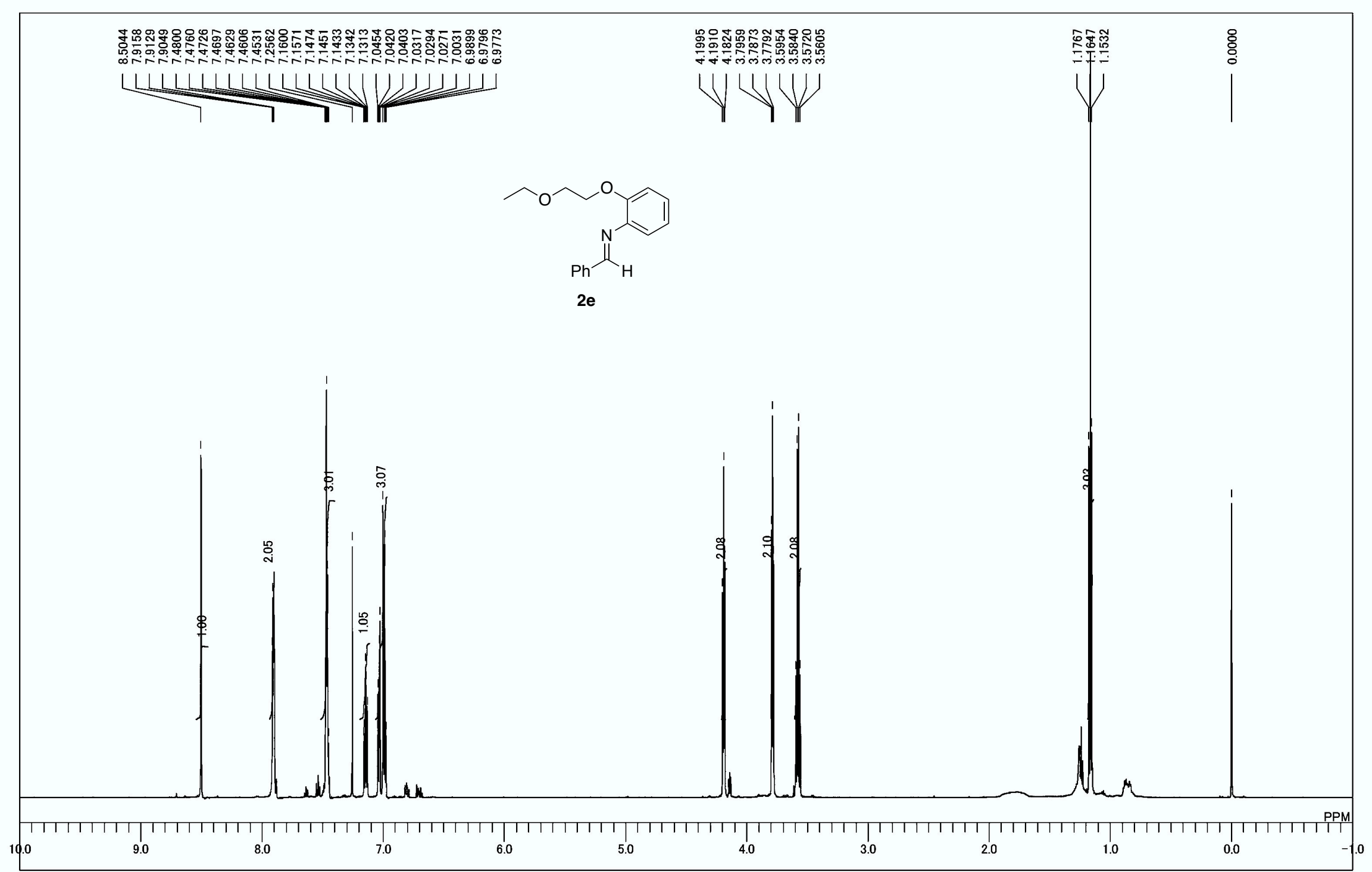

${ }^{1} \mathrm{H}$ NMR spectrum of $\mathbf{2 e}$ 


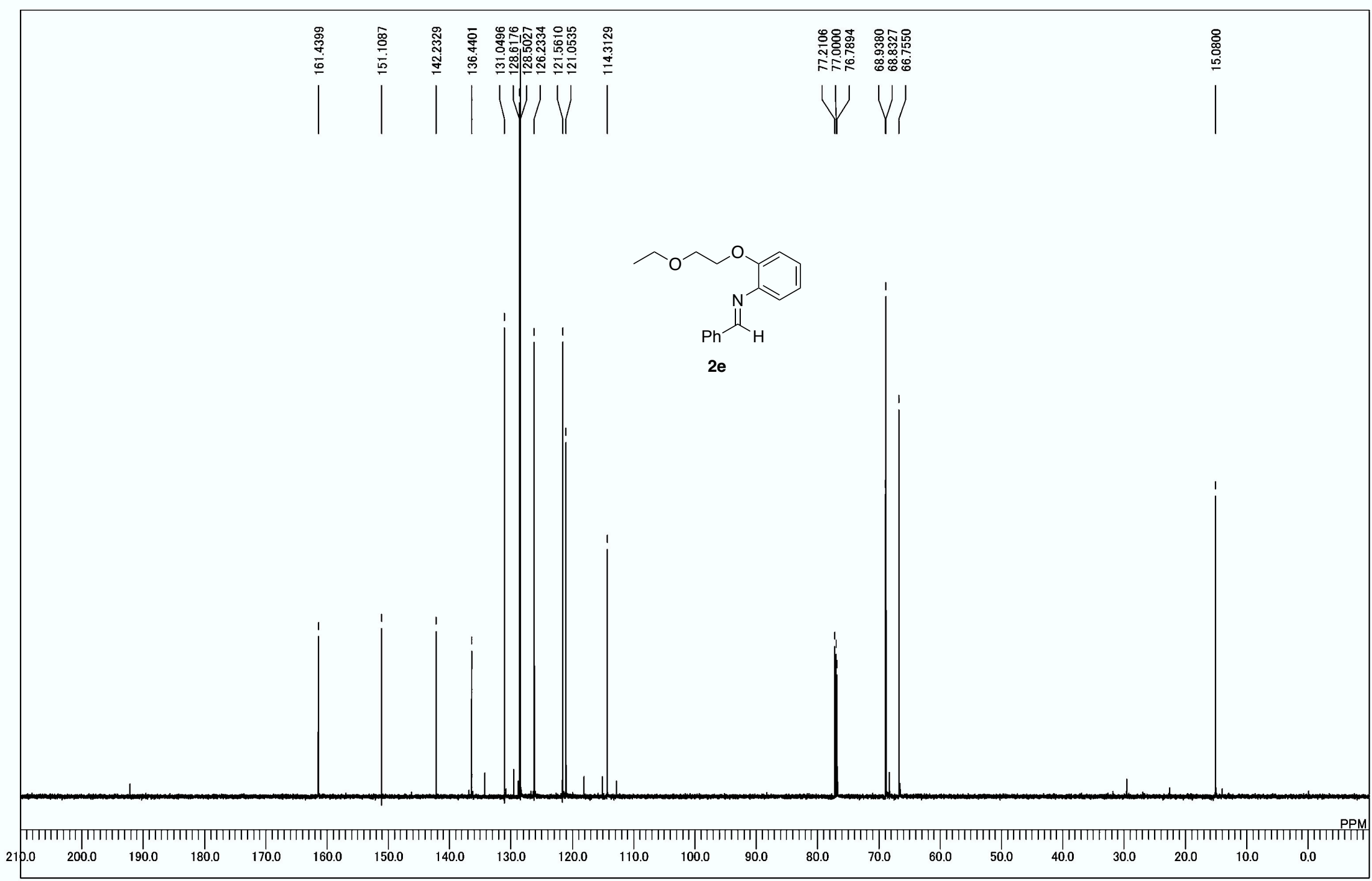

${ }^{13} \mathrm{C}$ NMR spectrum of $\mathbf{2 e}$ 


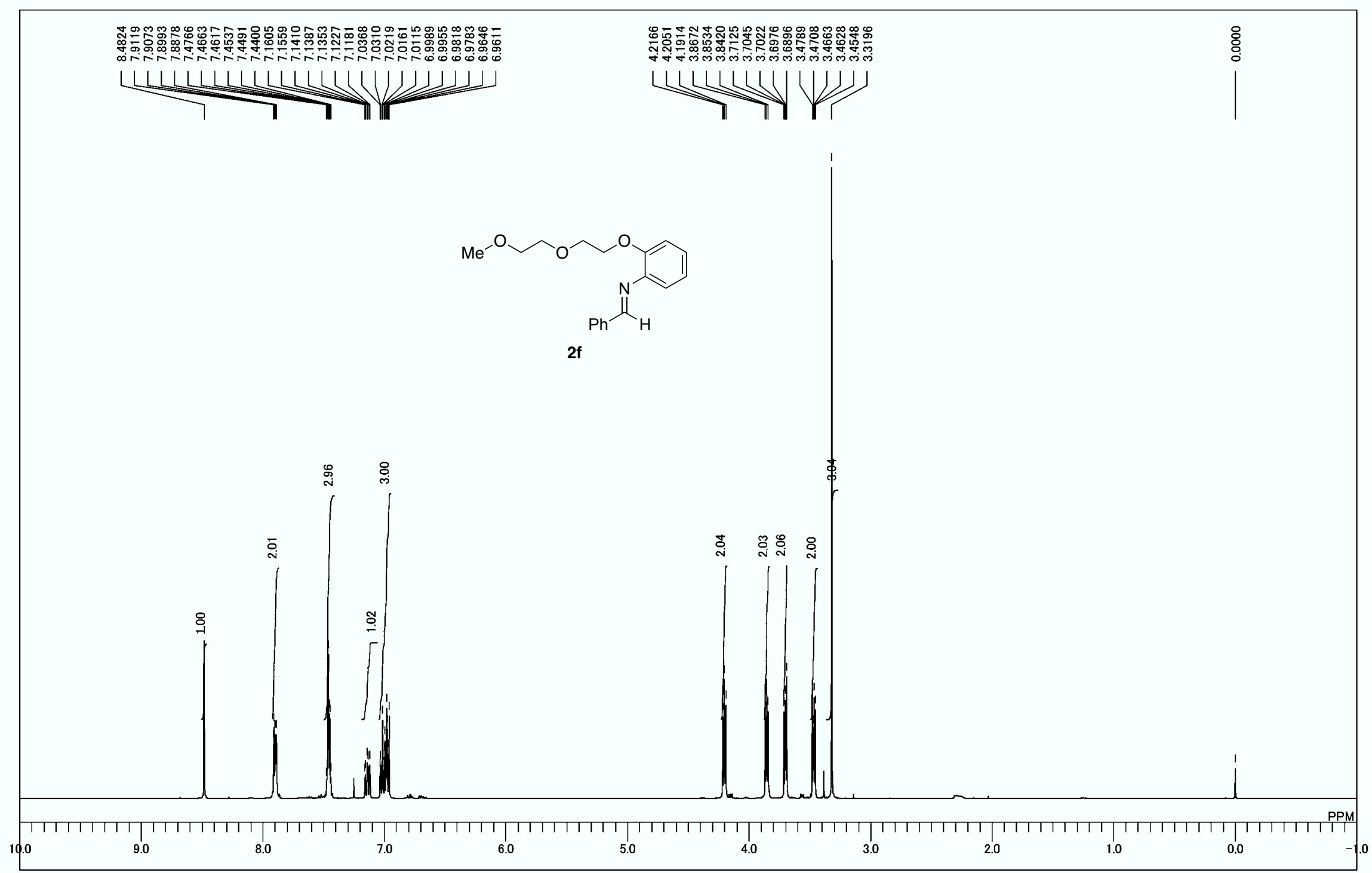

${ }^{1} \mathrm{H}$ NMR spectrum of $\mathbf{2 f}$ 


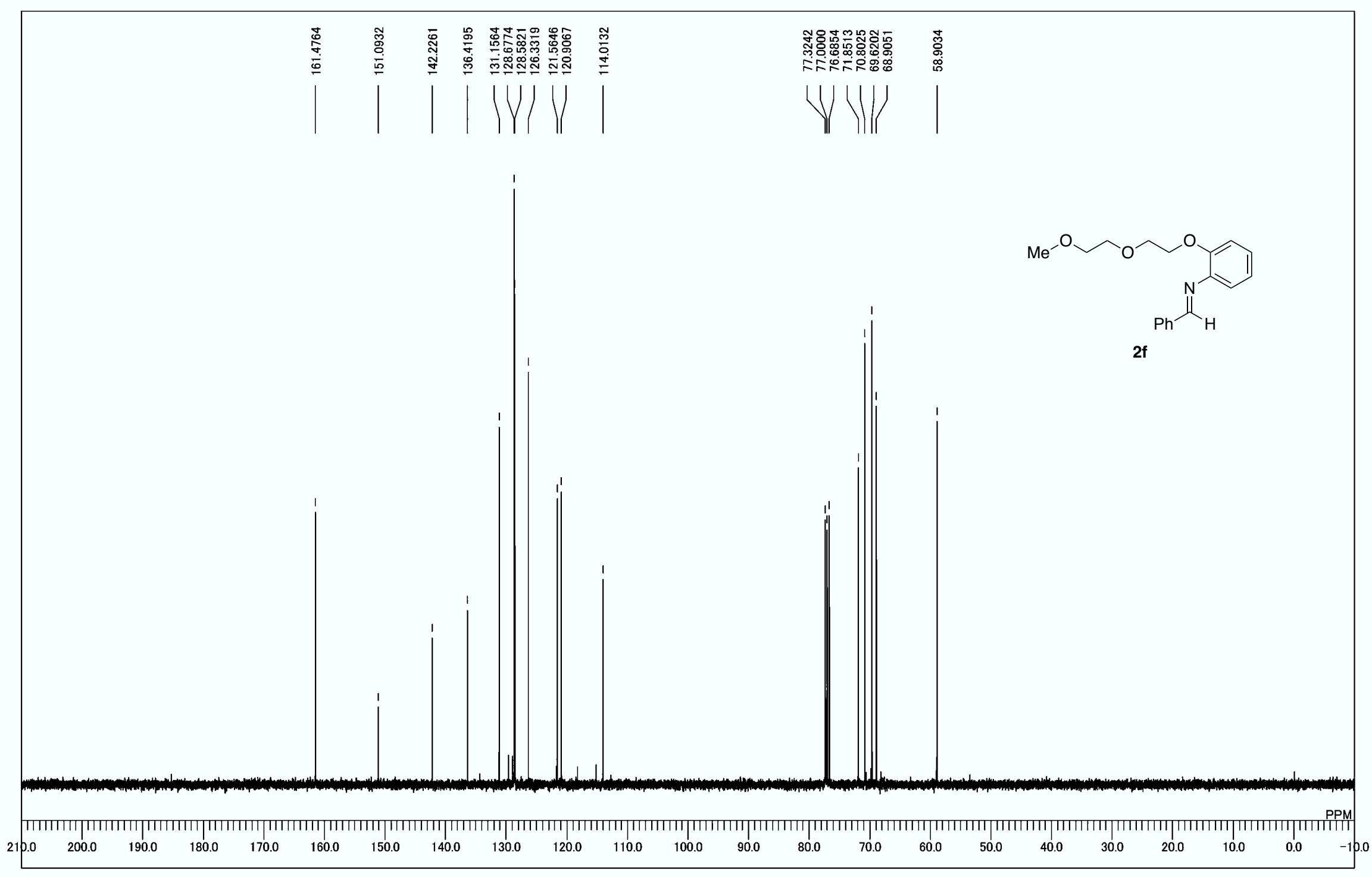

${ }^{13} \mathrm{C}$ NMR spectrum of $\mathbf{2 f}$ 


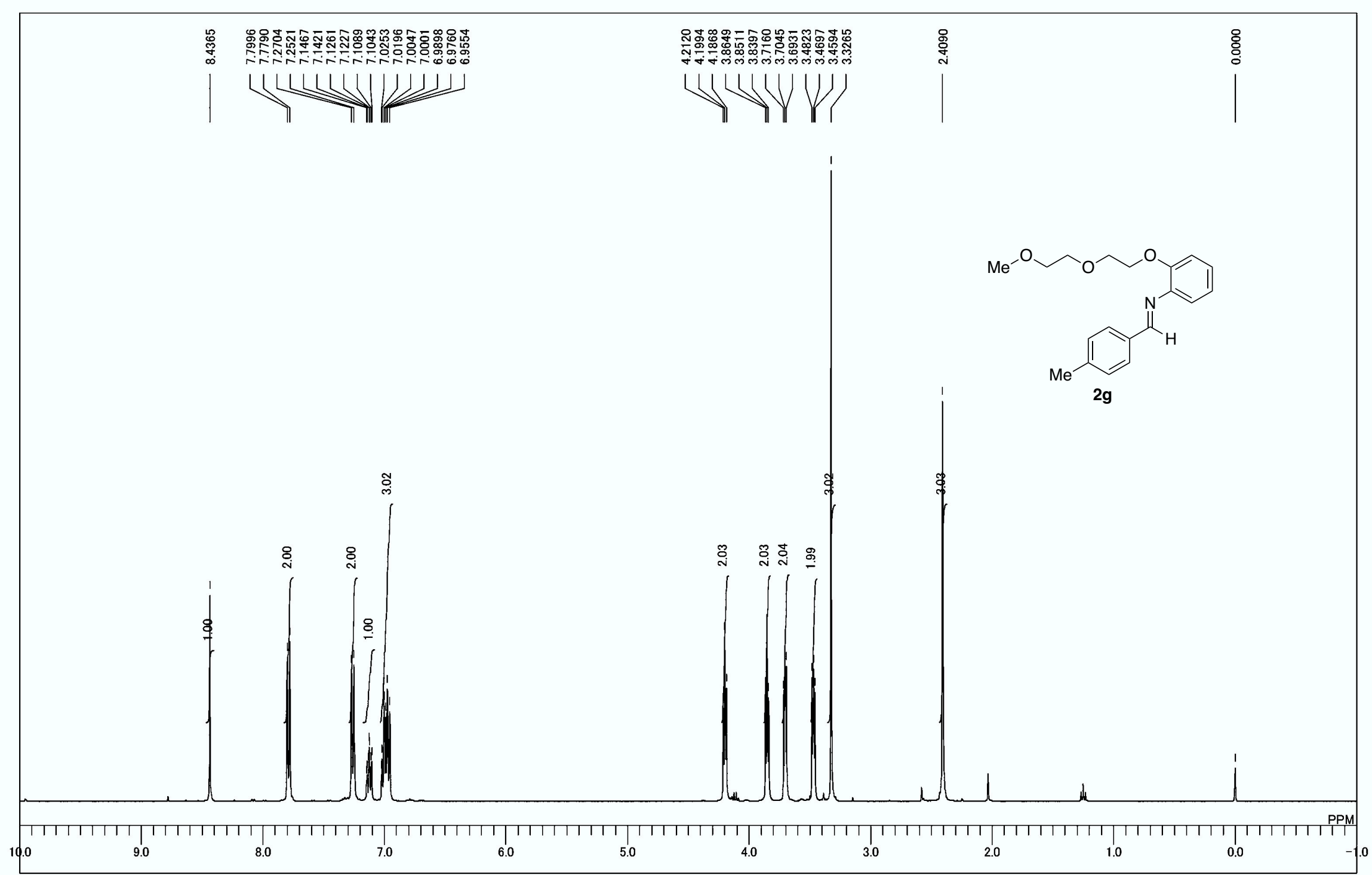

${ }^{1} \mathrm{H}$ NMR spectrum of $\mathbf{2 g}$ 


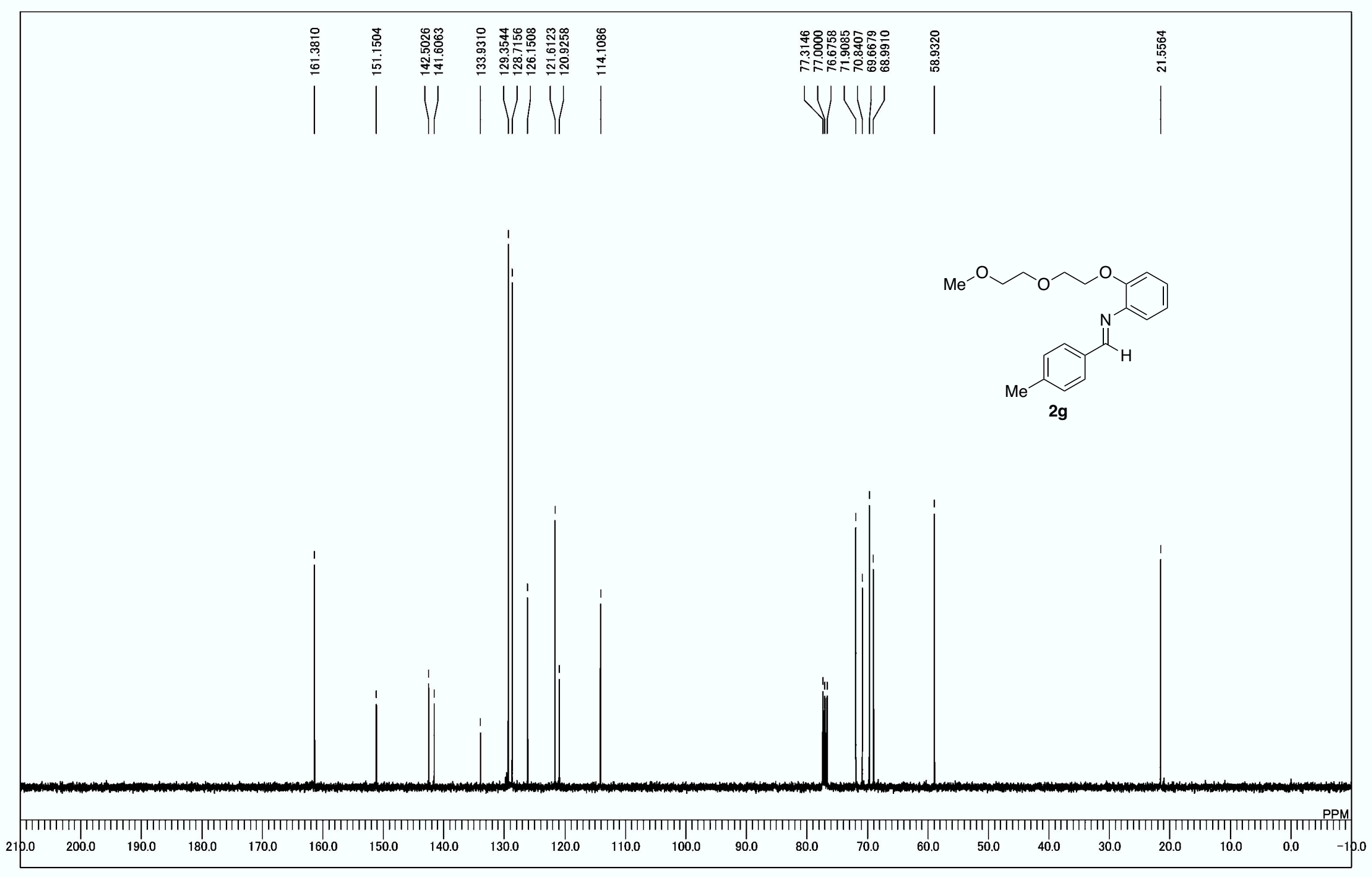

${ }^{13} \mathrm{C}$ NMR spectrum of $\mathbf{2 g}$ 


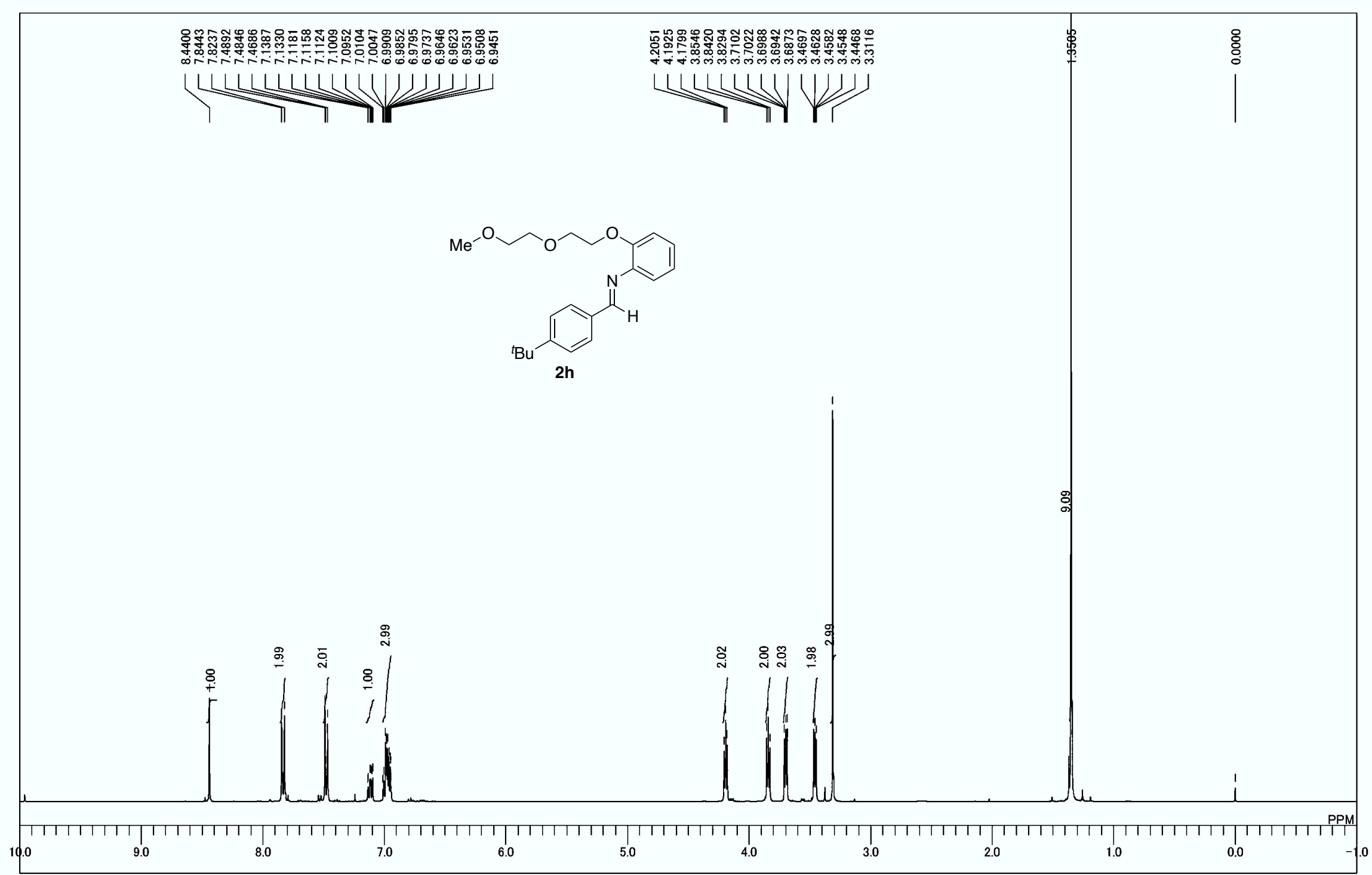

${ }^{1} \mathrm{H}$ NMR spectrum of $\mathbf{2 h}$ 


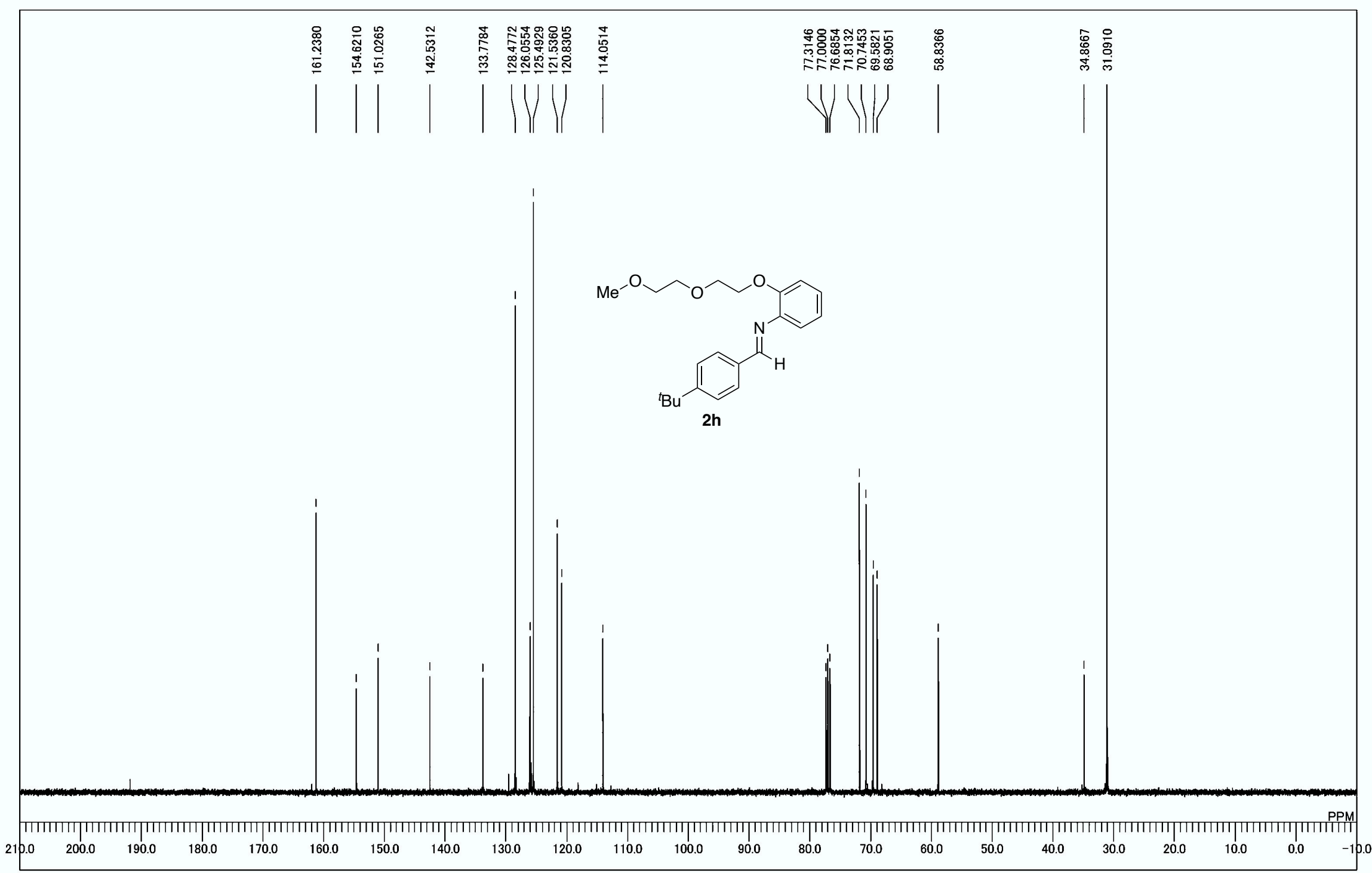

${ }^{13} \mathrm{C}$ NMR spectrum of $\mathbf{2 h}$ 


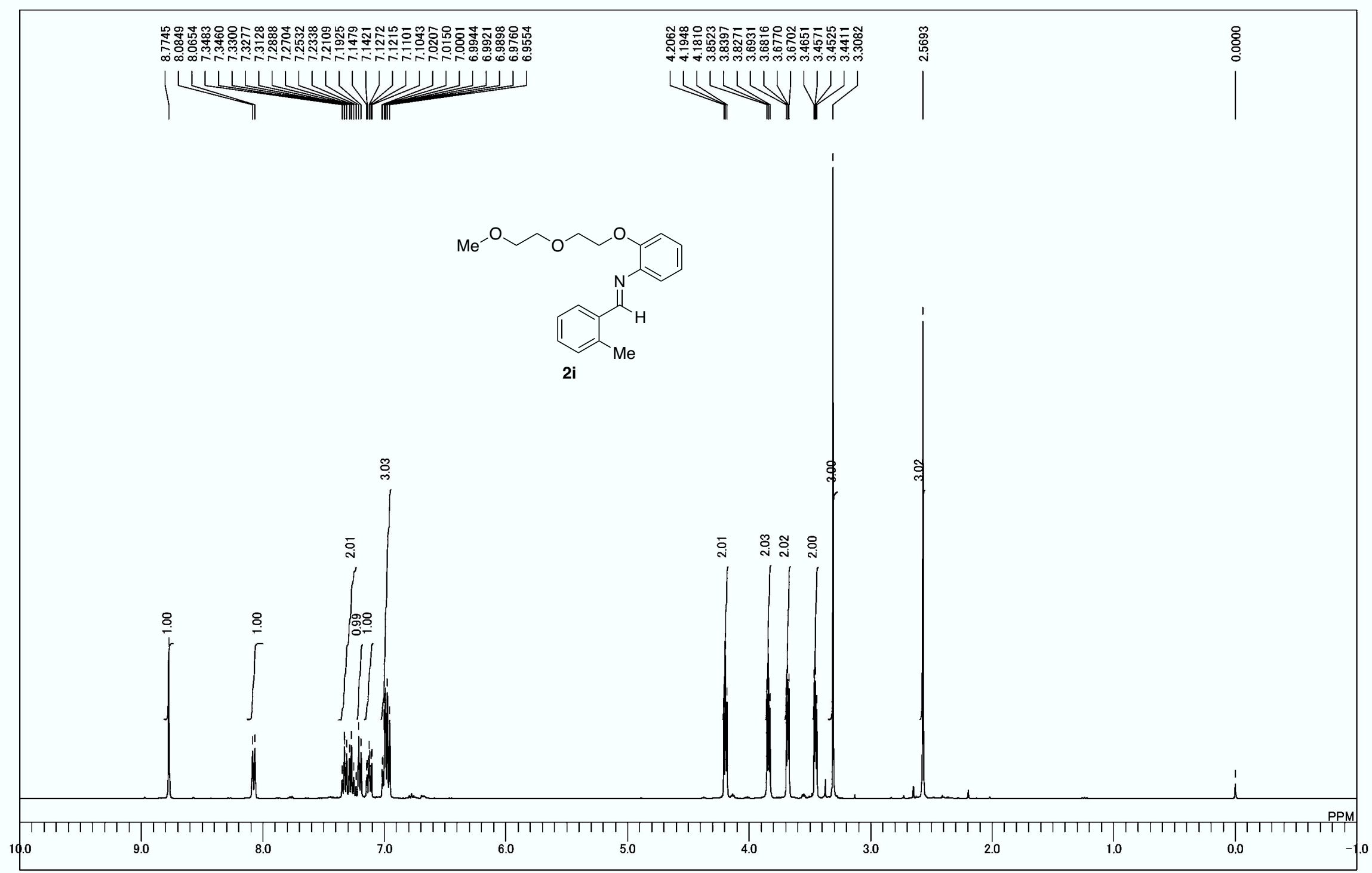

${ }^{1} \mathrm{H}$ NMR spectrum of $\mathbf{2 i}$ 


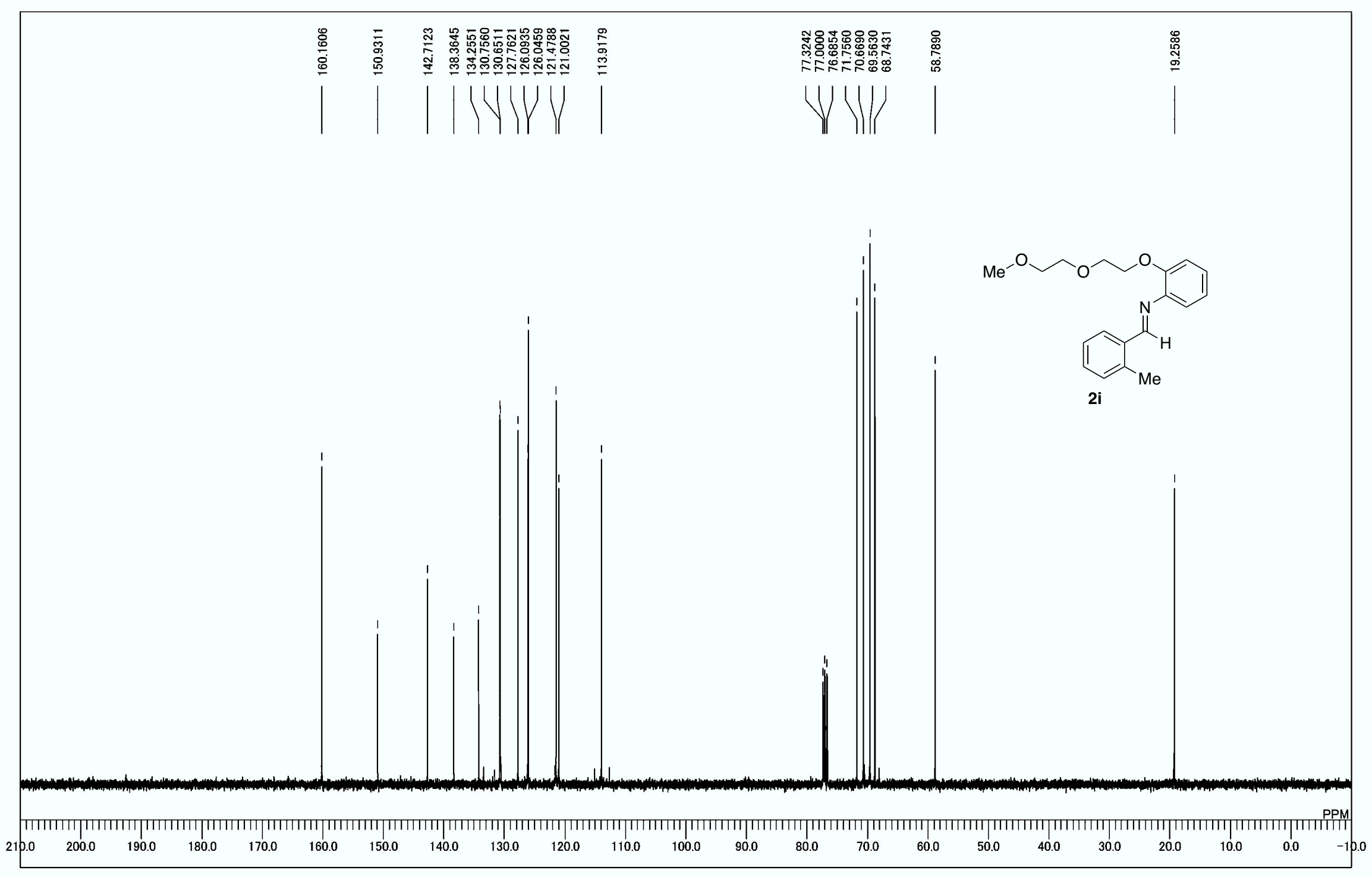

${ }^{13} \mathrm{C}$ NMR spectrum of $\mathbf{2 i}$ 


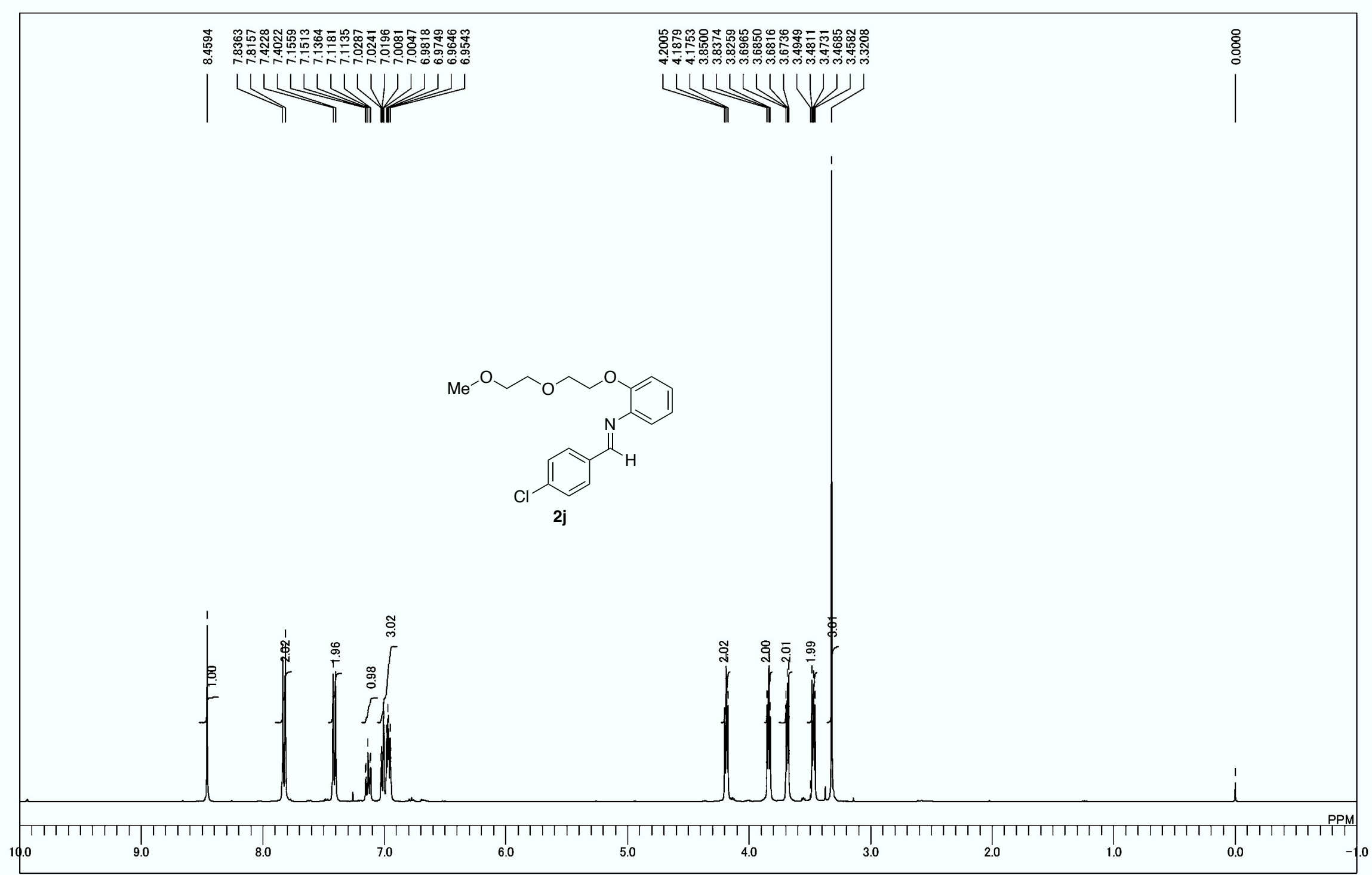

${ }^{1} \mathrm{H}$ NMR spectrum of $\mathbf{2} \mathbf{j}$ 


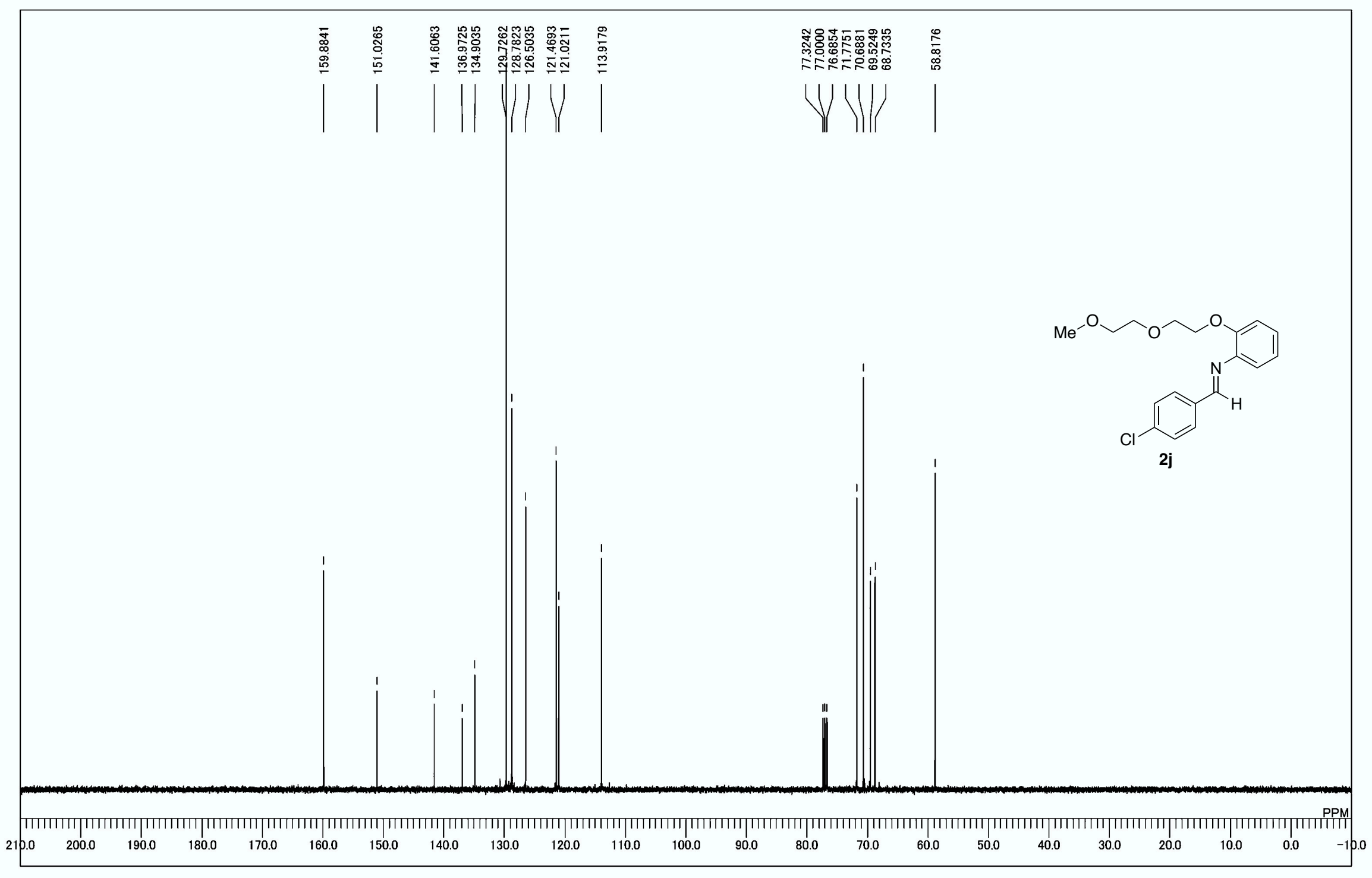

${ }^{13} \mathrm{C}$ NMR spectrum of $\mathbf{2} \mathbf{j}$ 


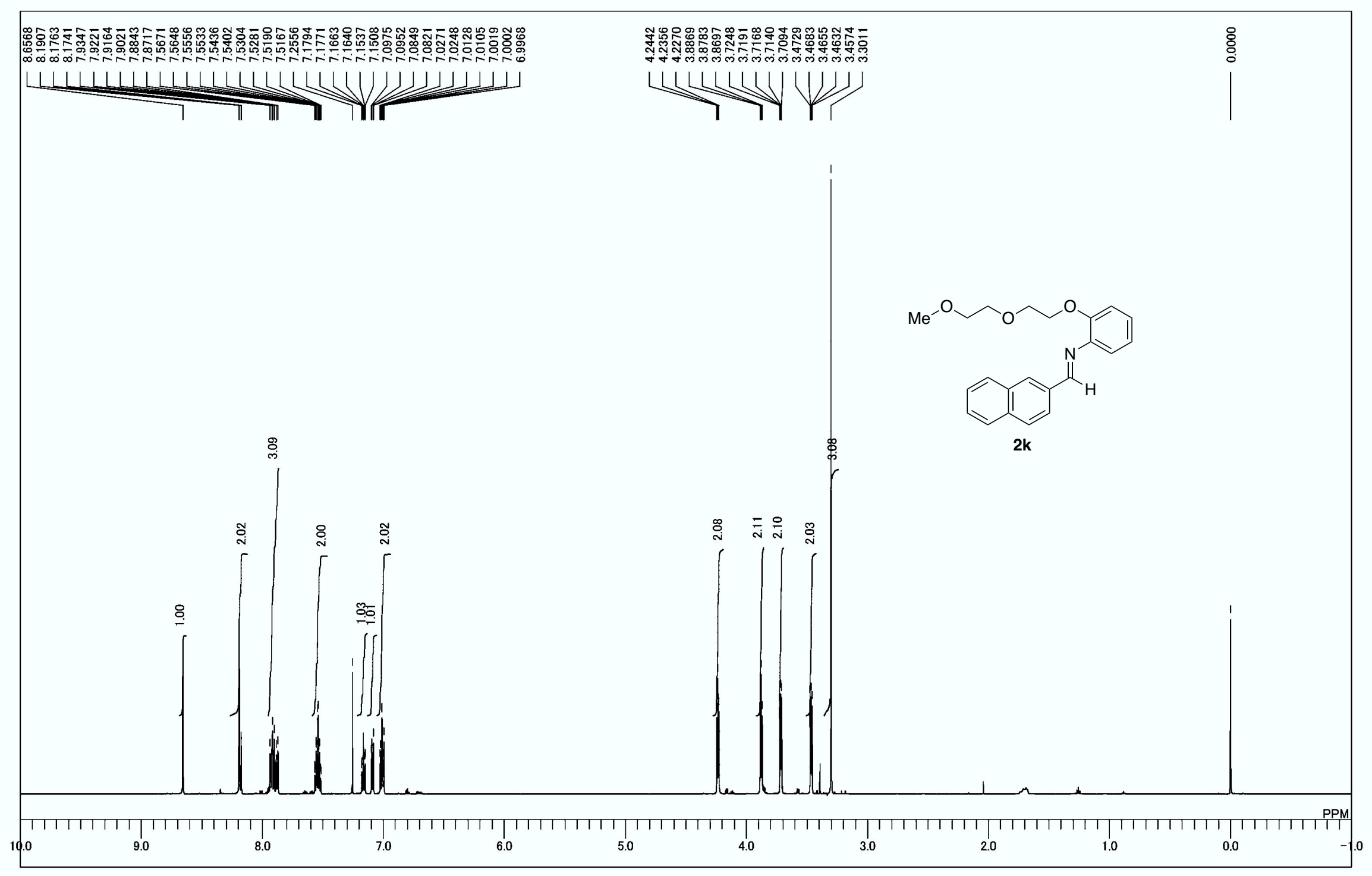

${ }^{1} \mathrm{H}$ NMR spectrum of $\mathbf{2} \mathbf{k}$ 


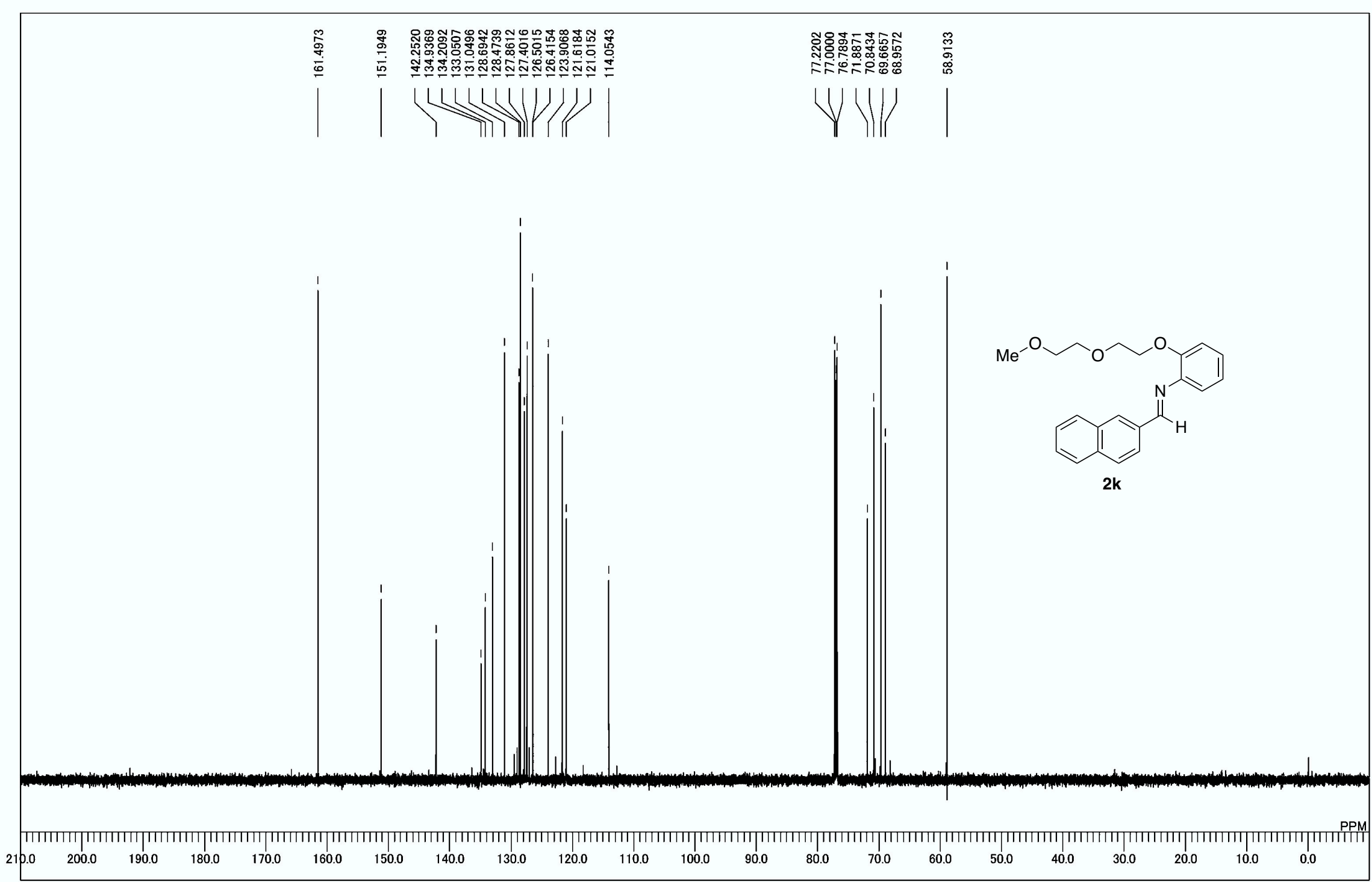

${ }^{13} \mathrm{C}$ NMR spectrum of $\mathbf{2 k}$ 


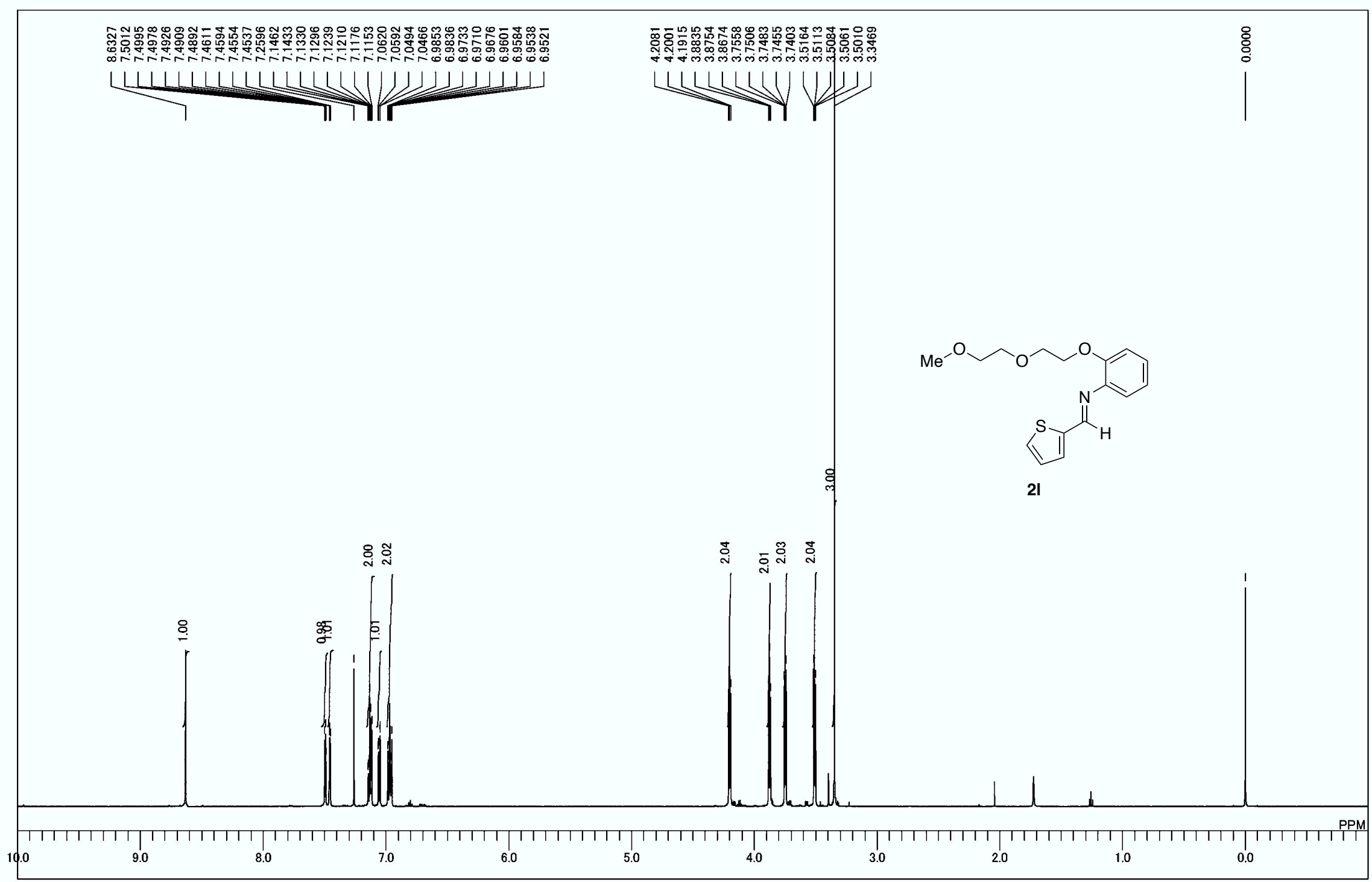

${ }^{1} \mathrm{H}$ NMR spectrum of $\mathbf{2 I}$ 


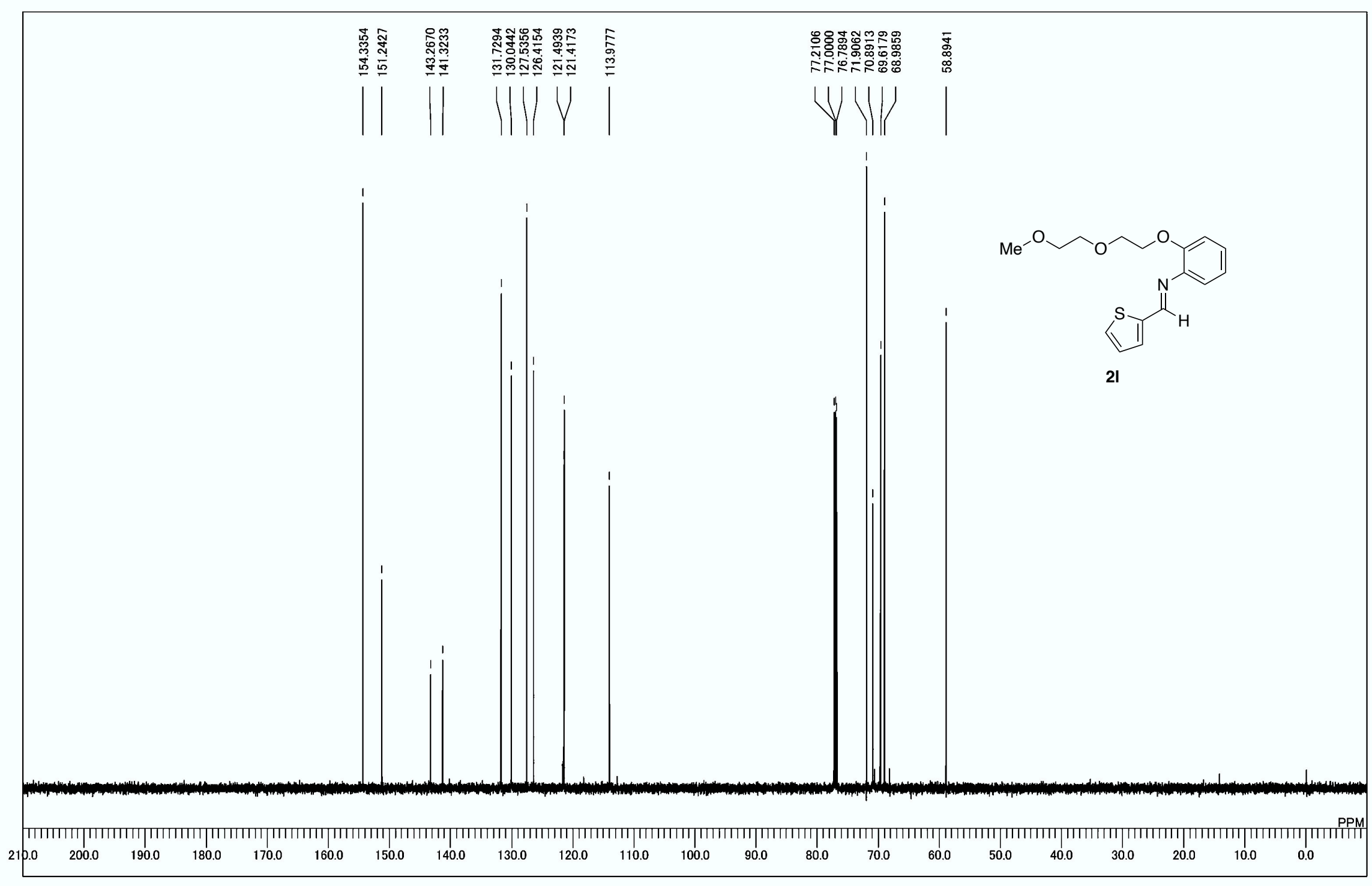

${ }^{13} \mathrm{C}$ NMR spectrum of $2 \mathrm{I}$ 


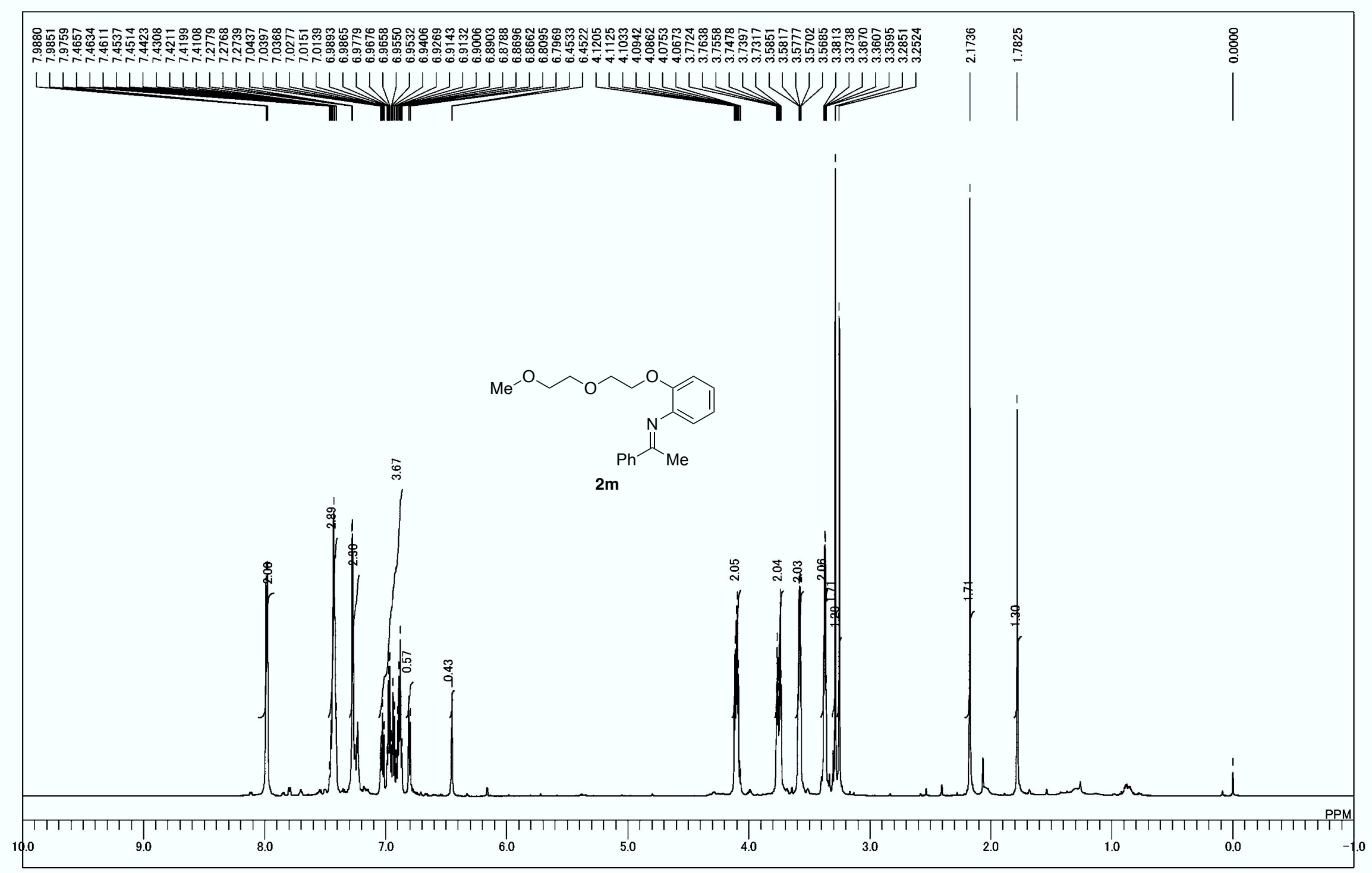

${ }^{1} \mathrm{H}$ NMR spectrum of $\mathbf{2 m}$ 


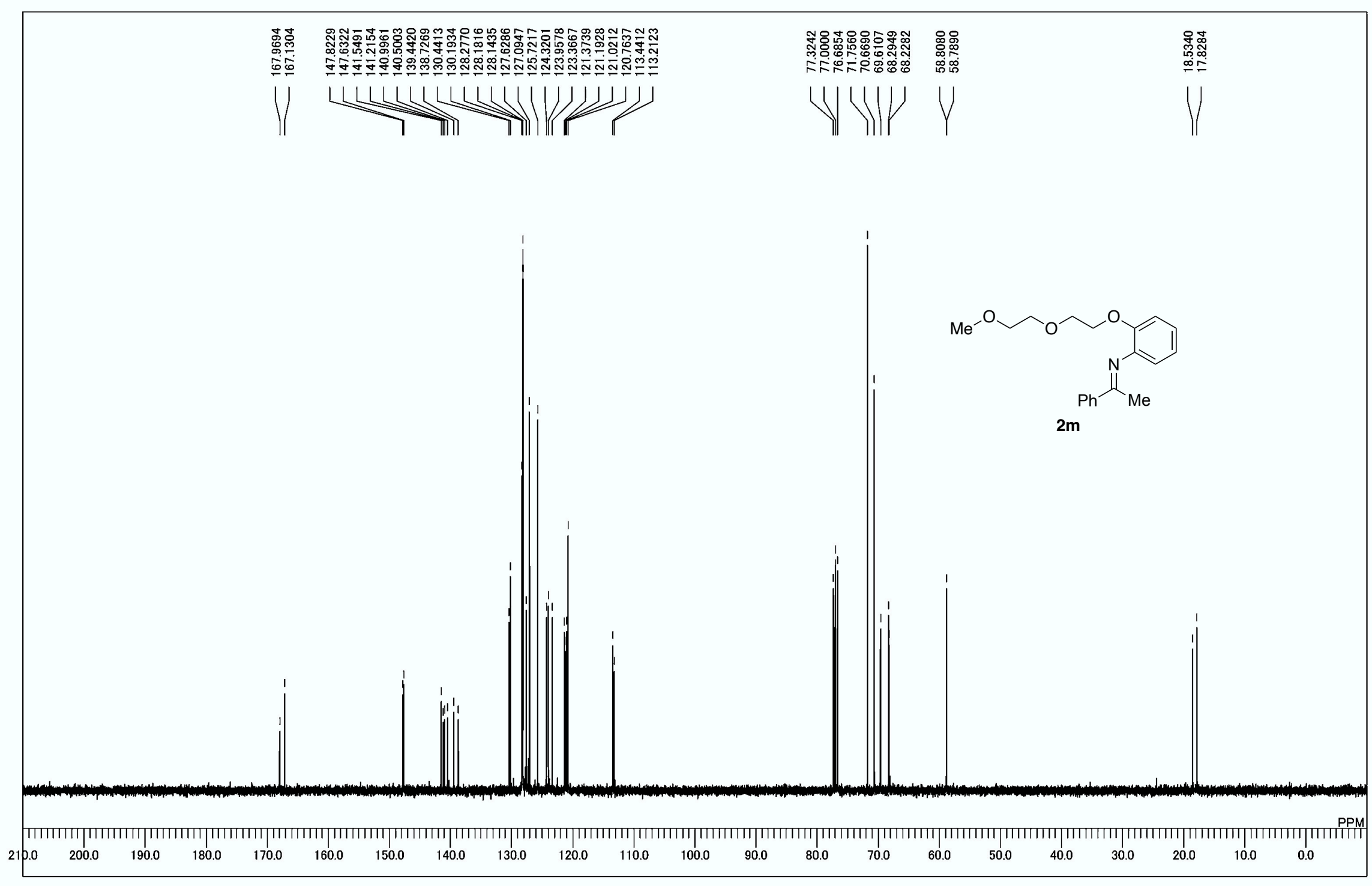

${ }^{13} \mathrm{C}$ NMR spectrum of $\mathbf{2 m}$ 


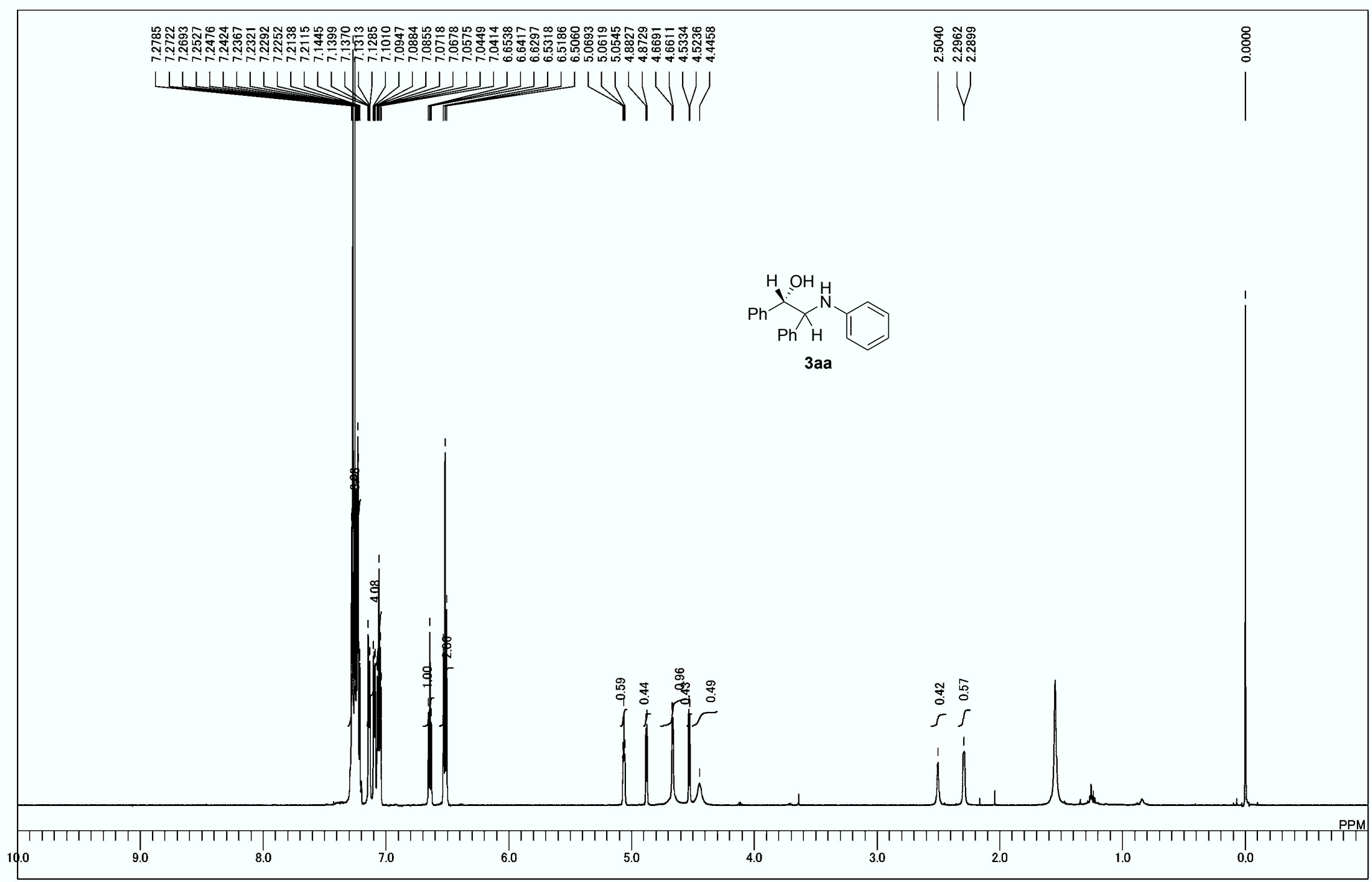

${ }^{1} \mathrm{H}$ NMR spectrum of $\mathbf{3 a a}$ 


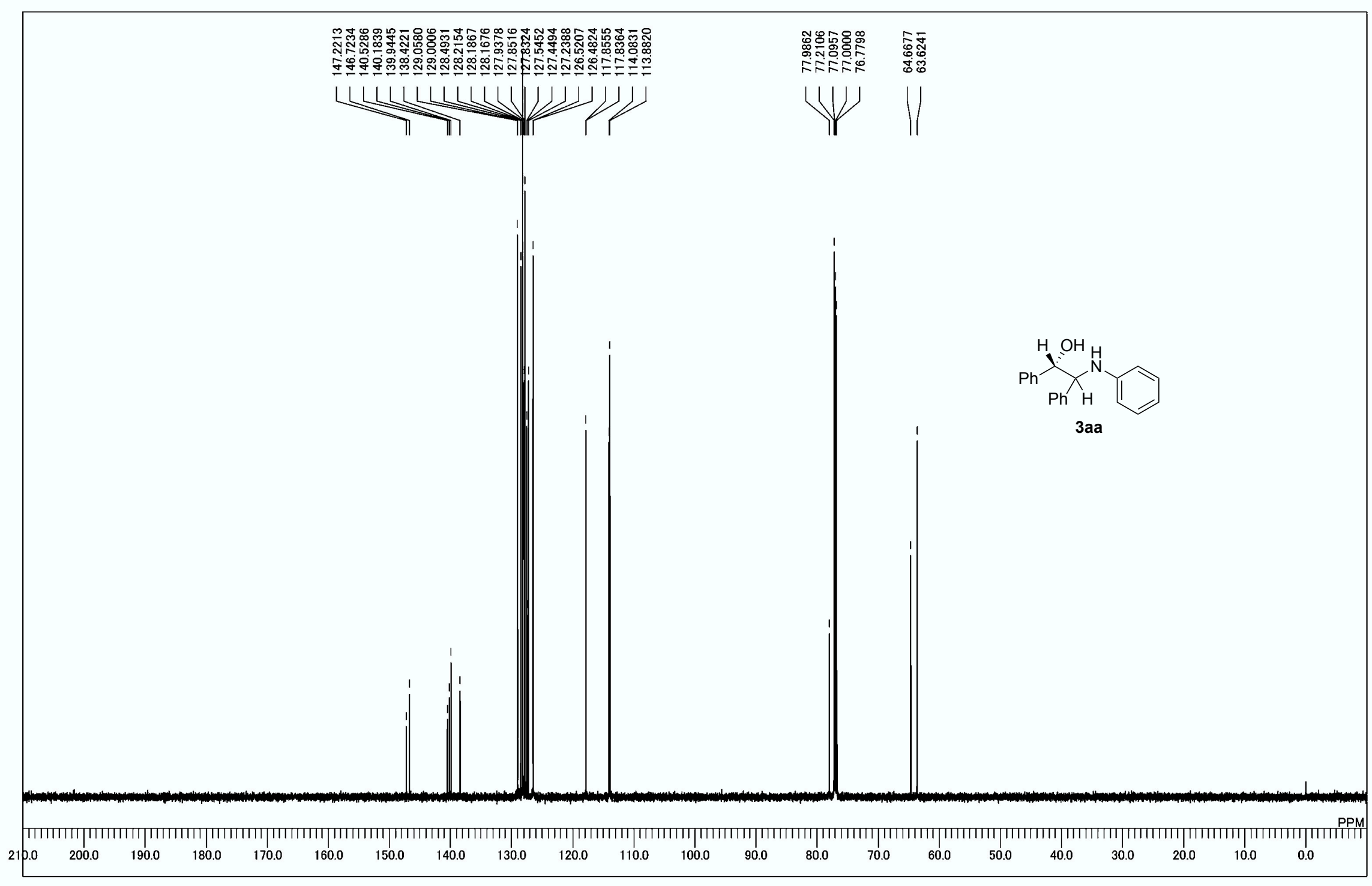

${ }^{13} \mathrm{C}$ NMR spectrum of $\mathbf{3 a a}$ 


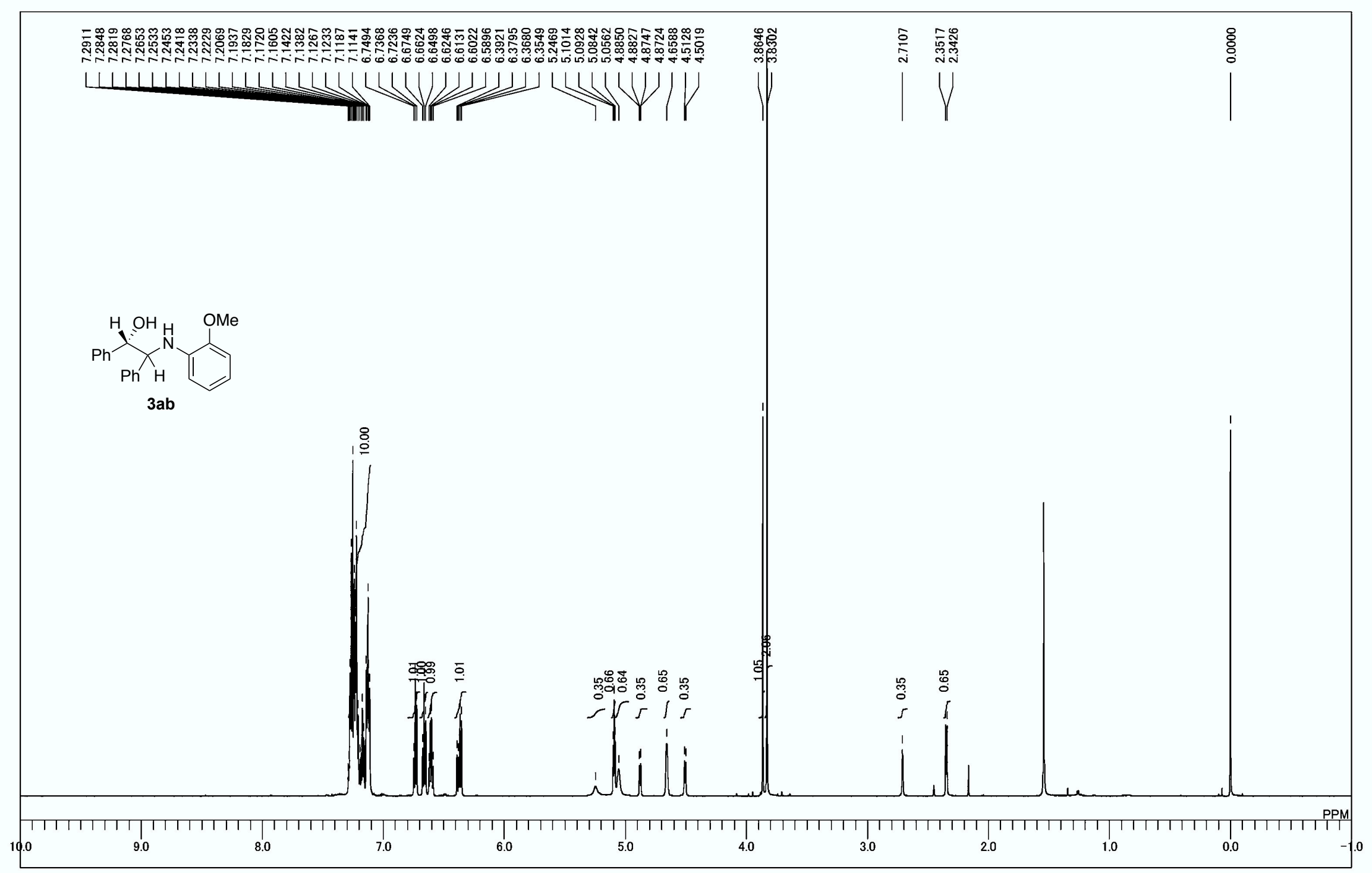

${ }^{1} \mathrm{H}$ NMR spectrum of $\mathbf{3 a b}$ 


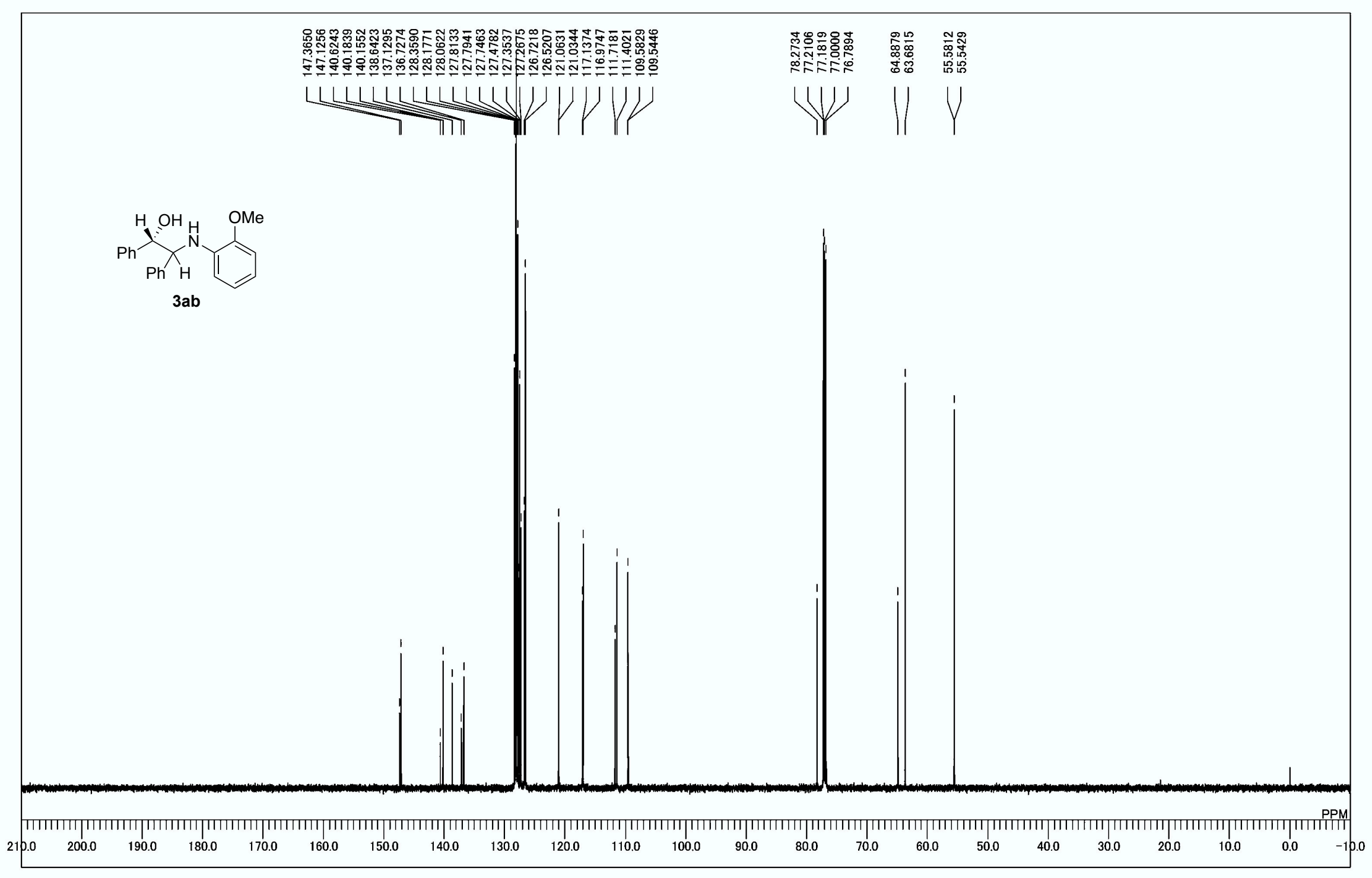

${ }^{13} \mathrm{C}$ NMR spectrum of $\mathbf{3 a b}$ 


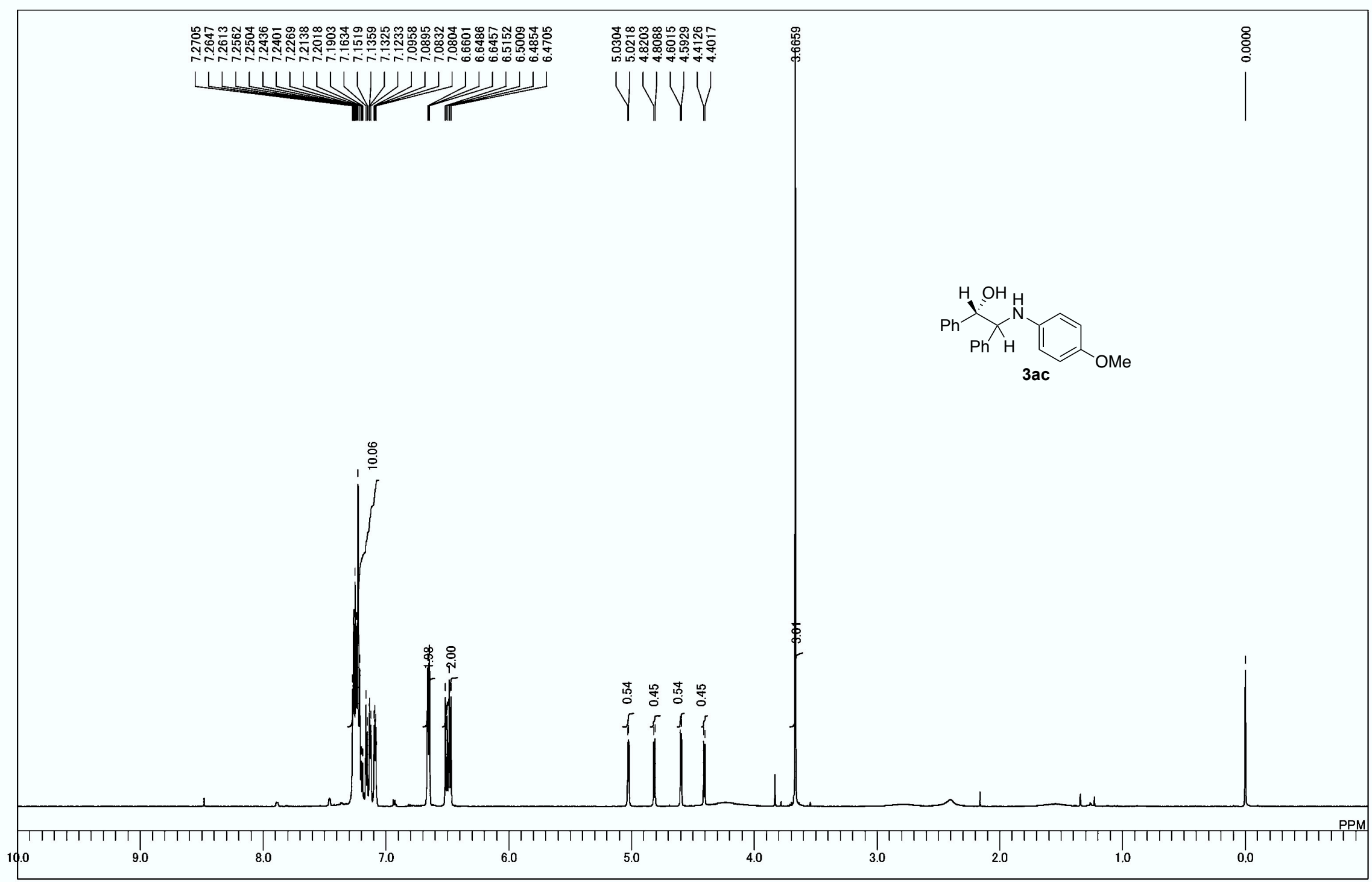

${ }^{1} \mathrm{H}$ NMR spectrum of $\mathbf{3 a c}$ 


$$
=\sqrt{n+\infty}
$$




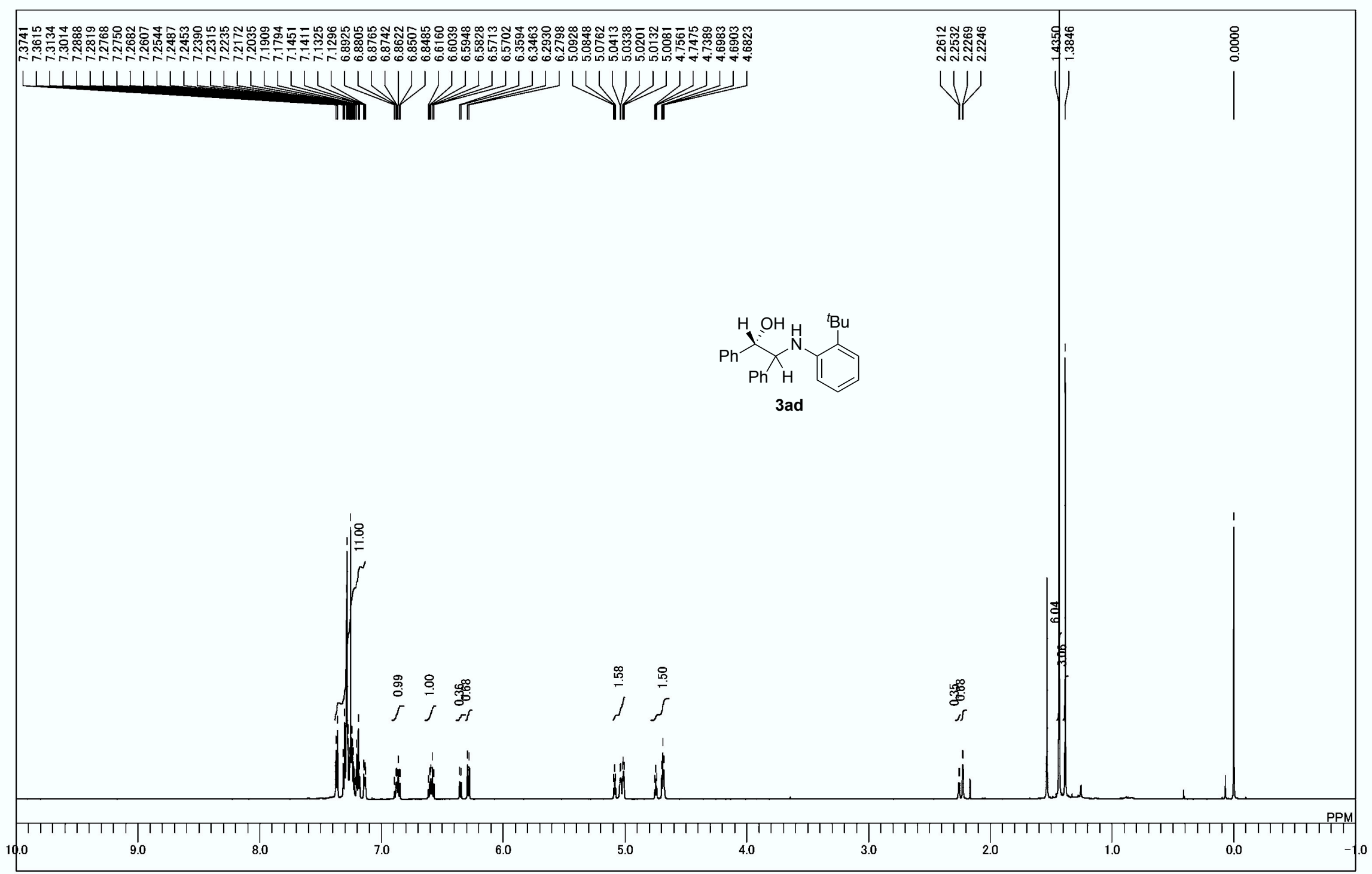

${ }^{1} \mathrm{H}$ NMR spectrum of $\mathbf{3 a d}$ 


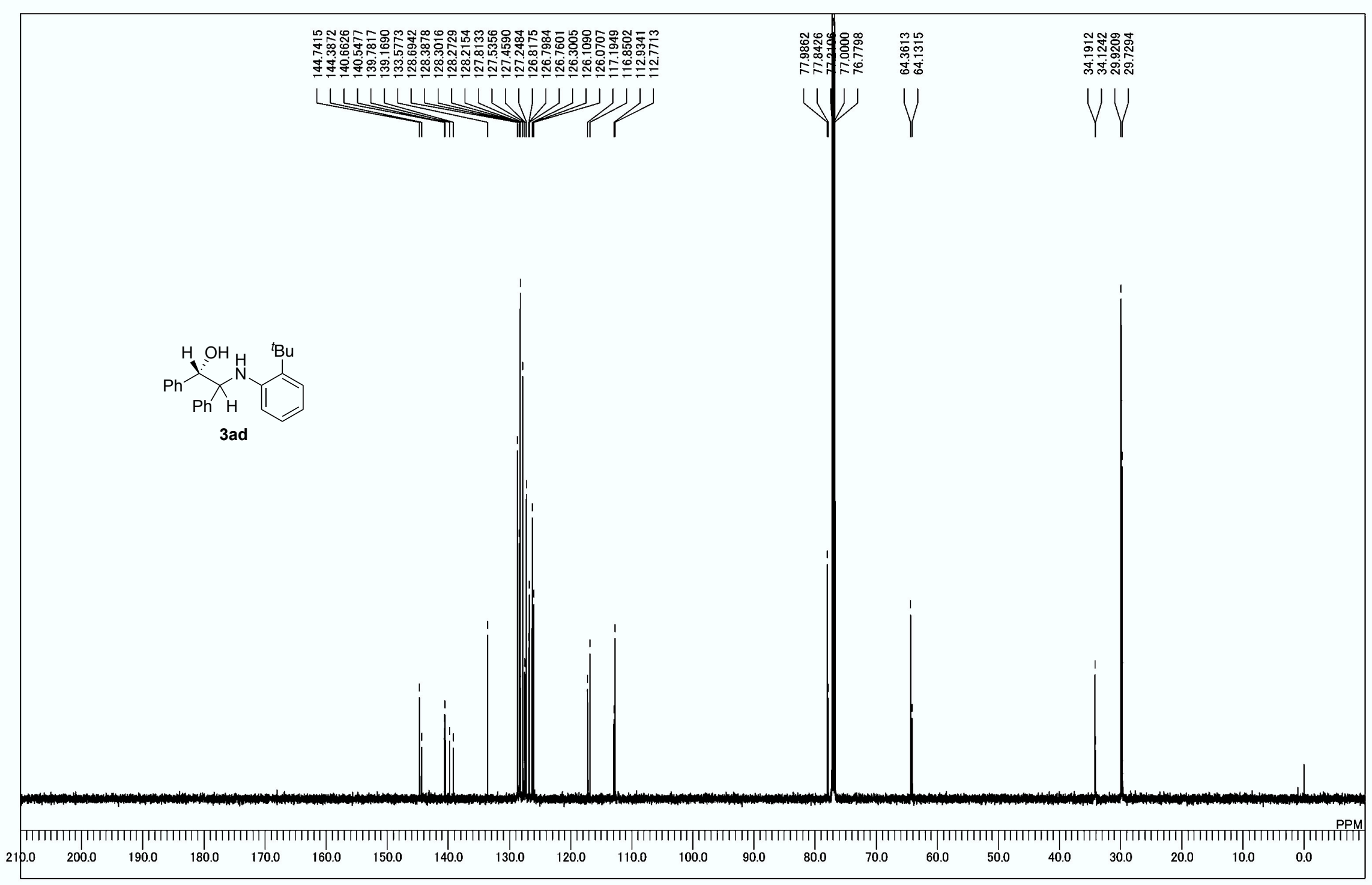

${ }^{13} \mathrm{C}$ NMR spectrum of $\mathbf{3 a d}$ 


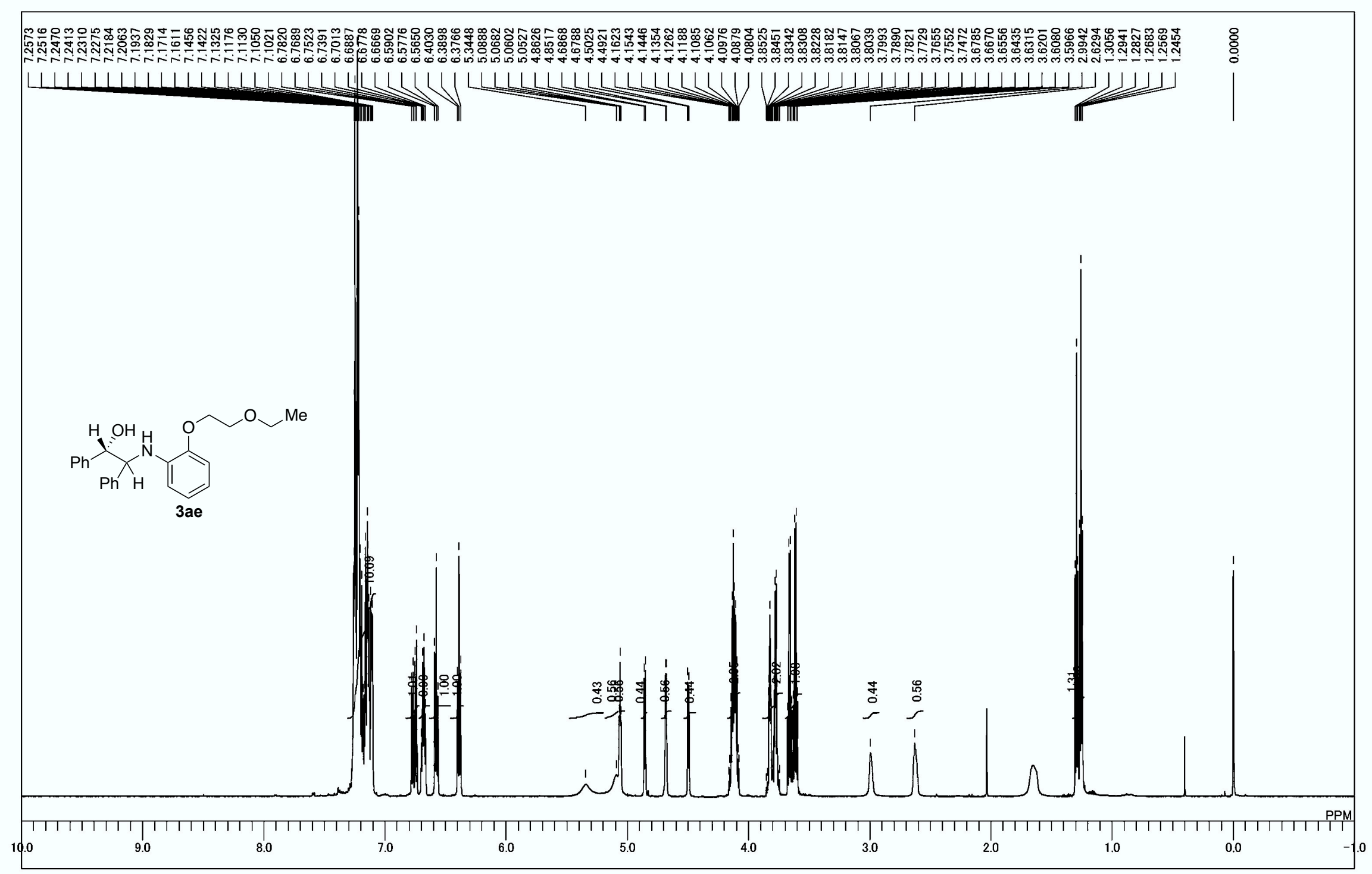

${ }^{1} \mathrm{H}$ NMR spectrum of $\mathbf{3 a e}$ 


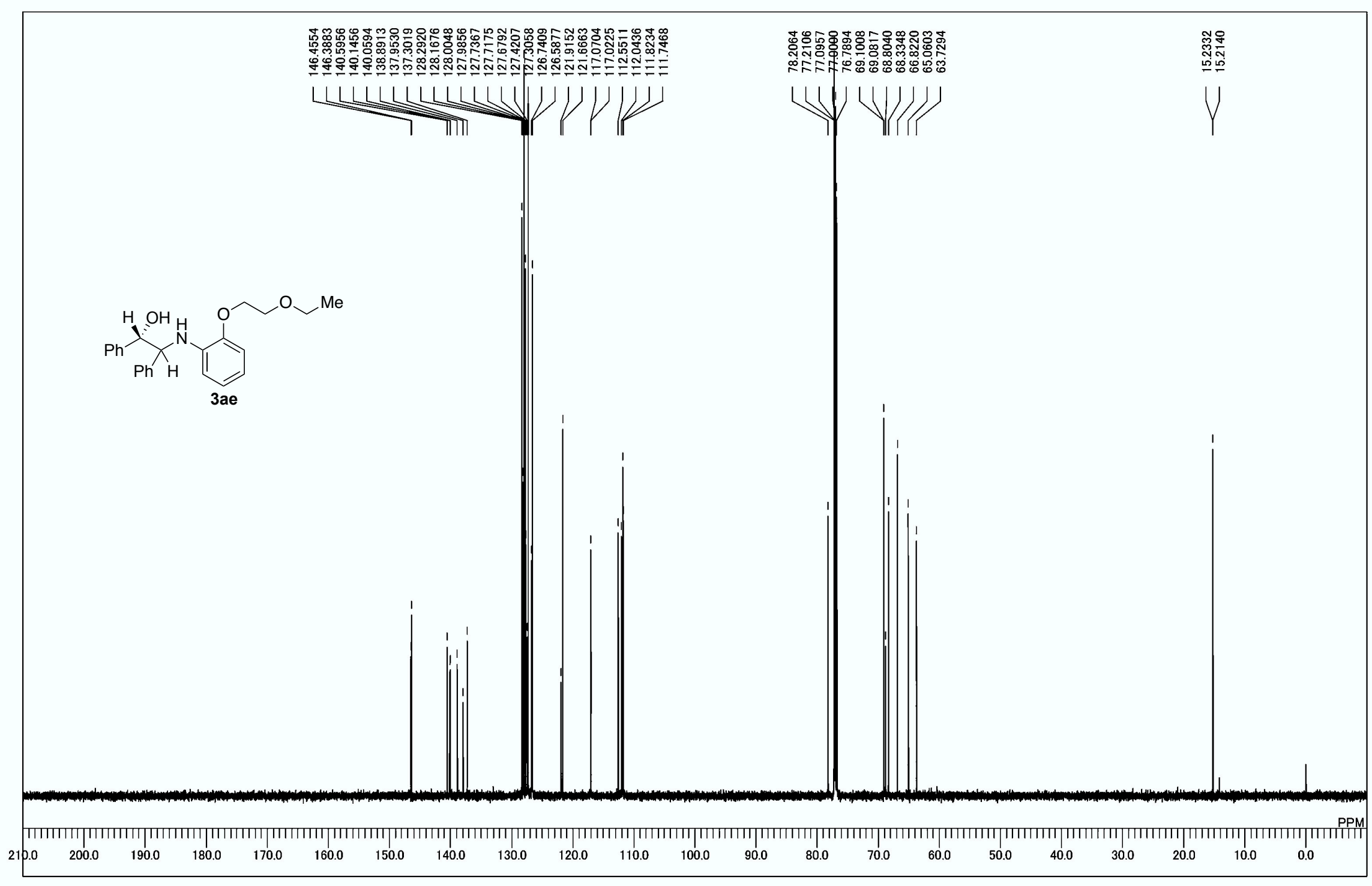

${ }^{13} \mathrm{C}$ NMR spectrum of $\mathbf{3 a e}$ 


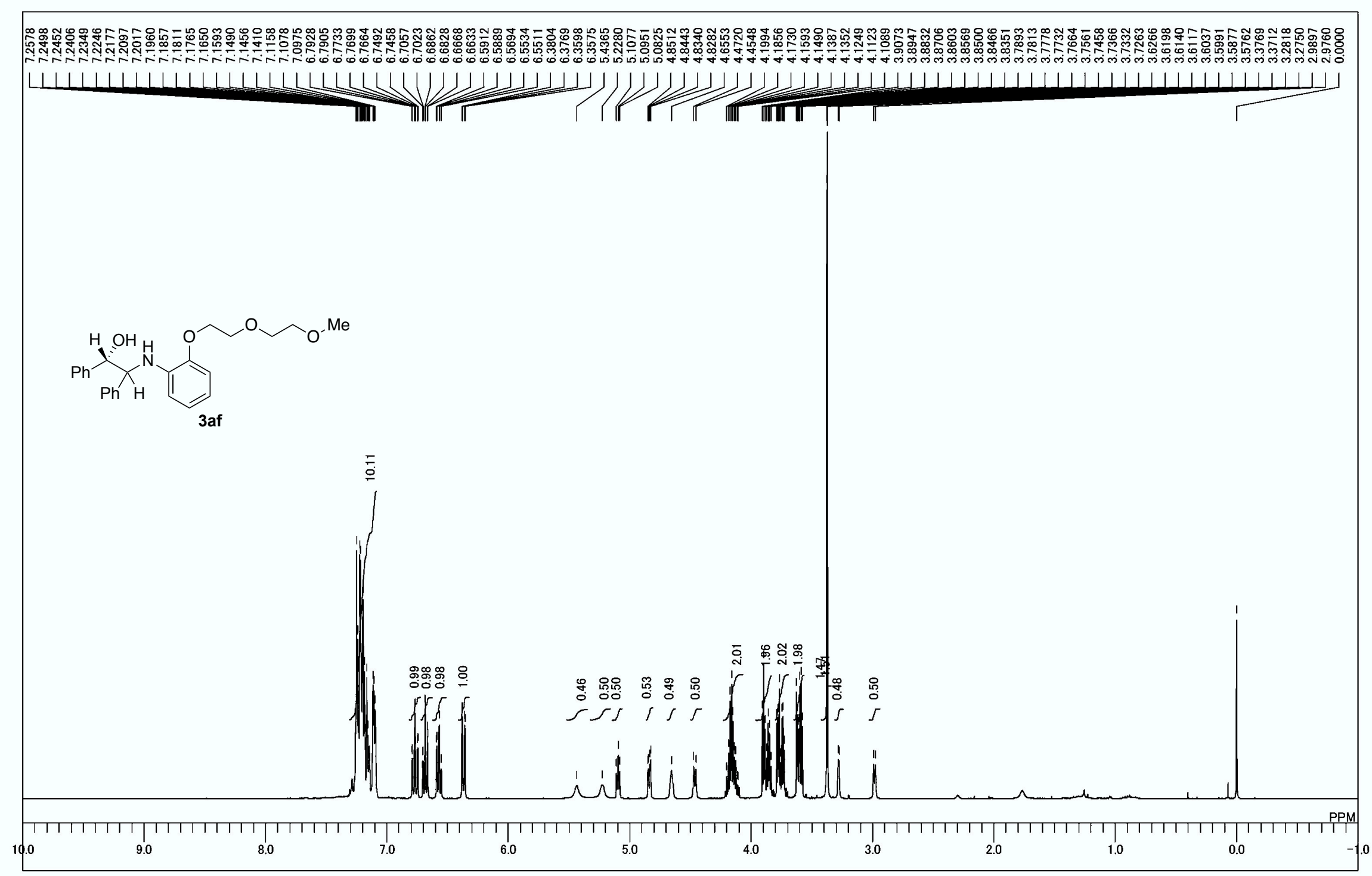

${ }^{1} \mathrm{H}$ NMR spectrum of $\mathbf{3 a f}$ 


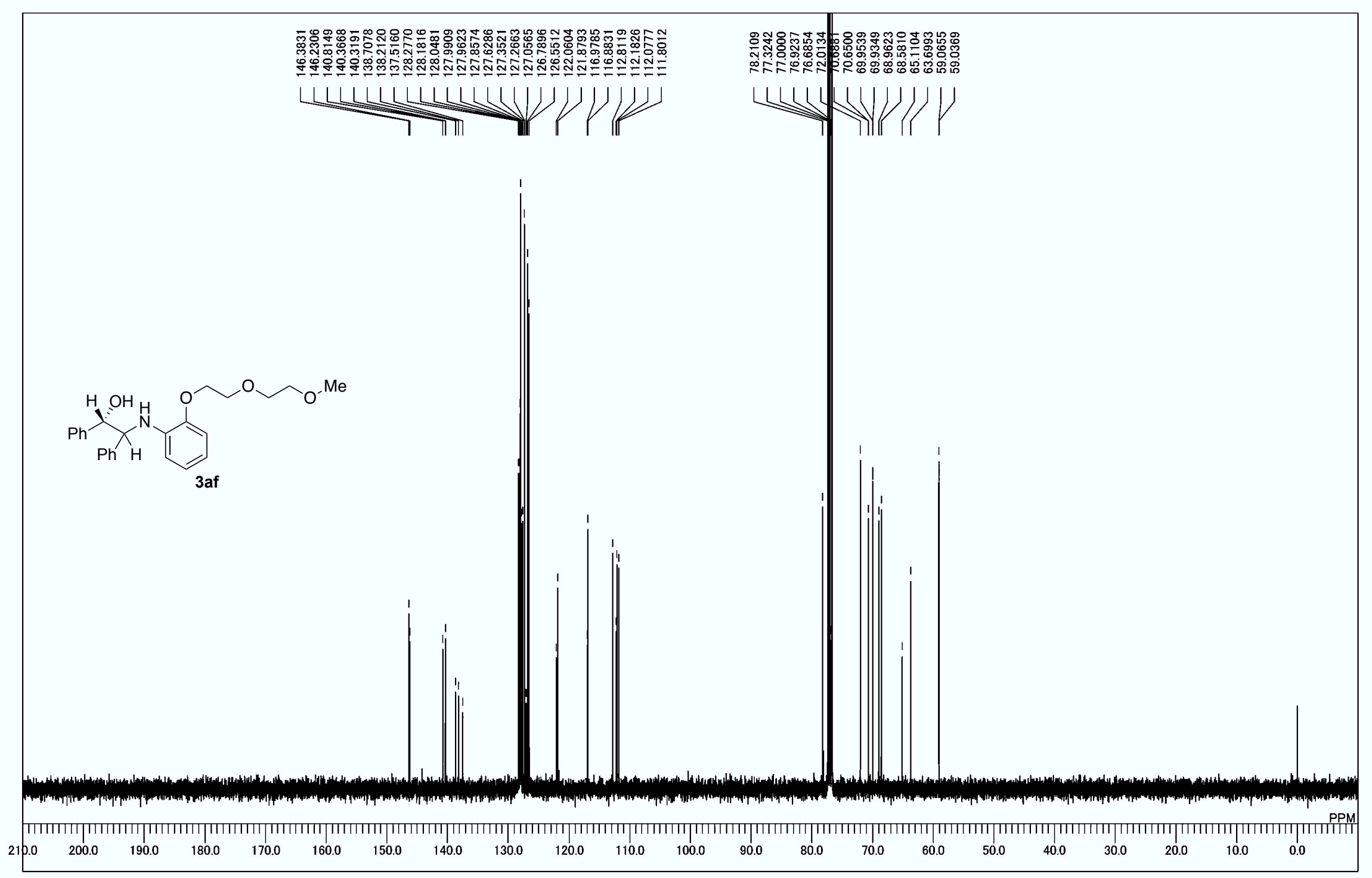

${ }^{13} \mathrm{C}$ NMR spectrum of $\mathbf{3 a f}$ 


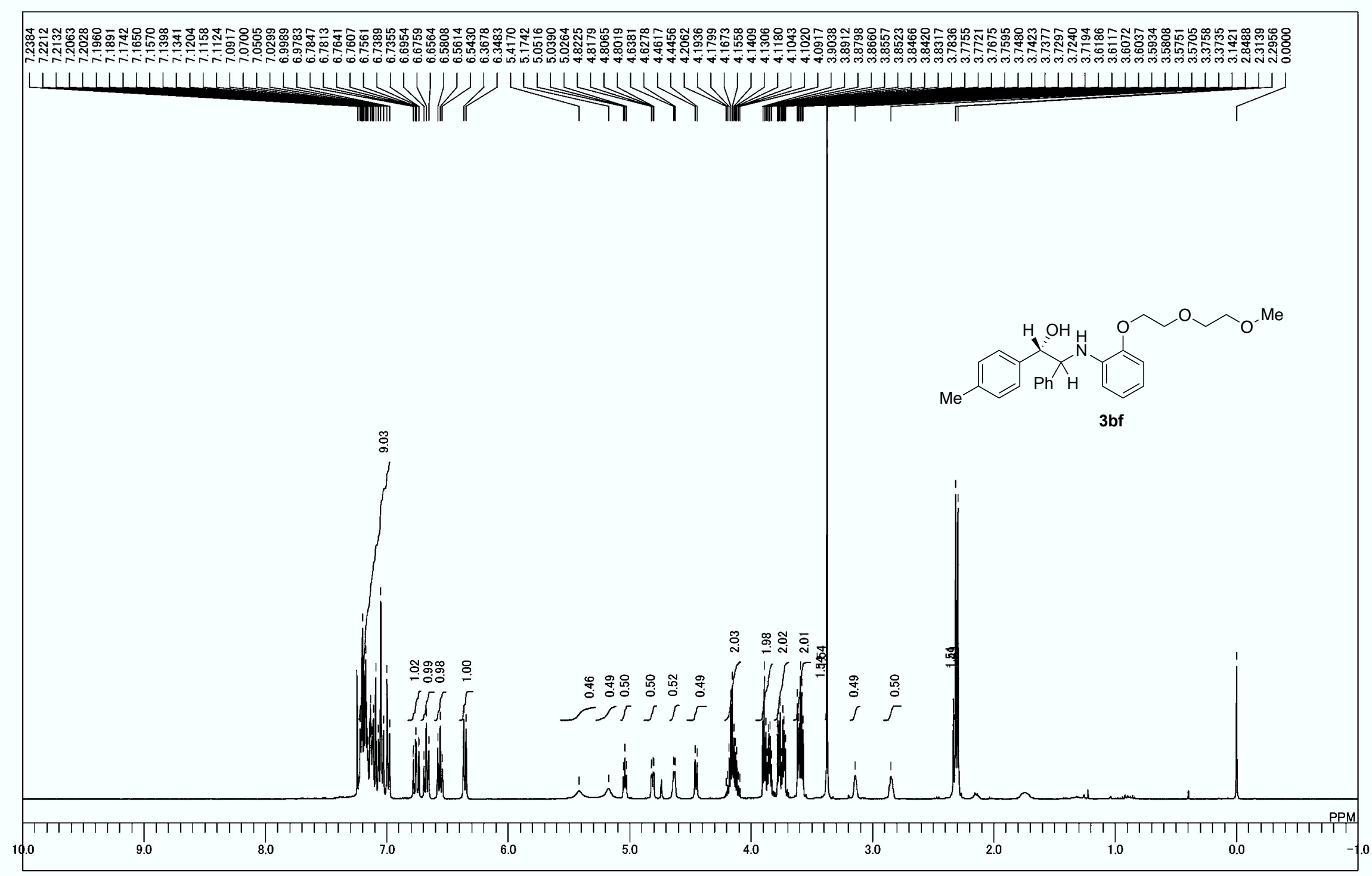

${ }^{1} \mathrm{H}$ NMR spectrum of $\mathbf{3 b f}$ 


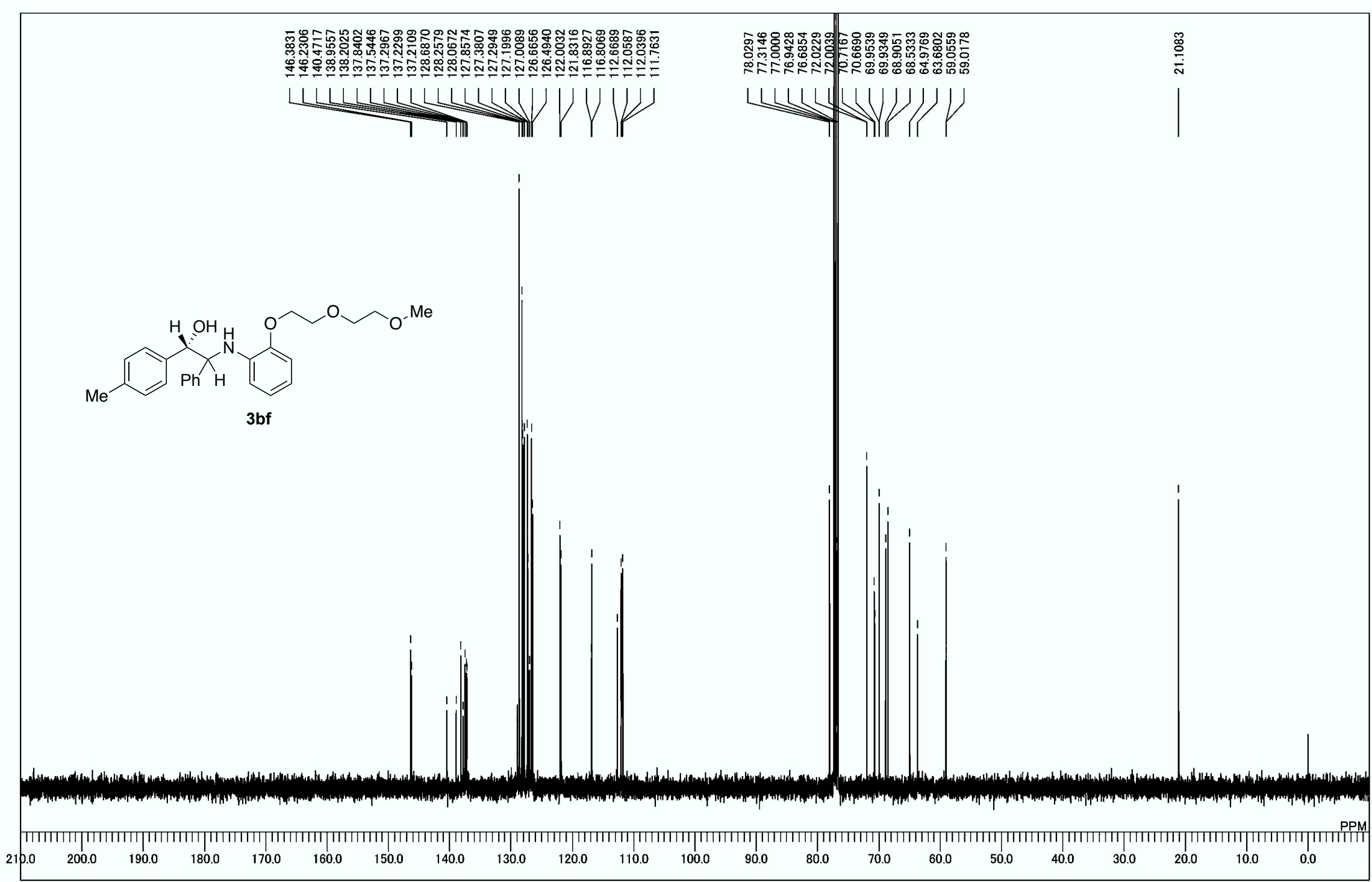

${ }^{13} \mathrm{C}$ NMR spectrum of $\mathbf{3 b f}$ 


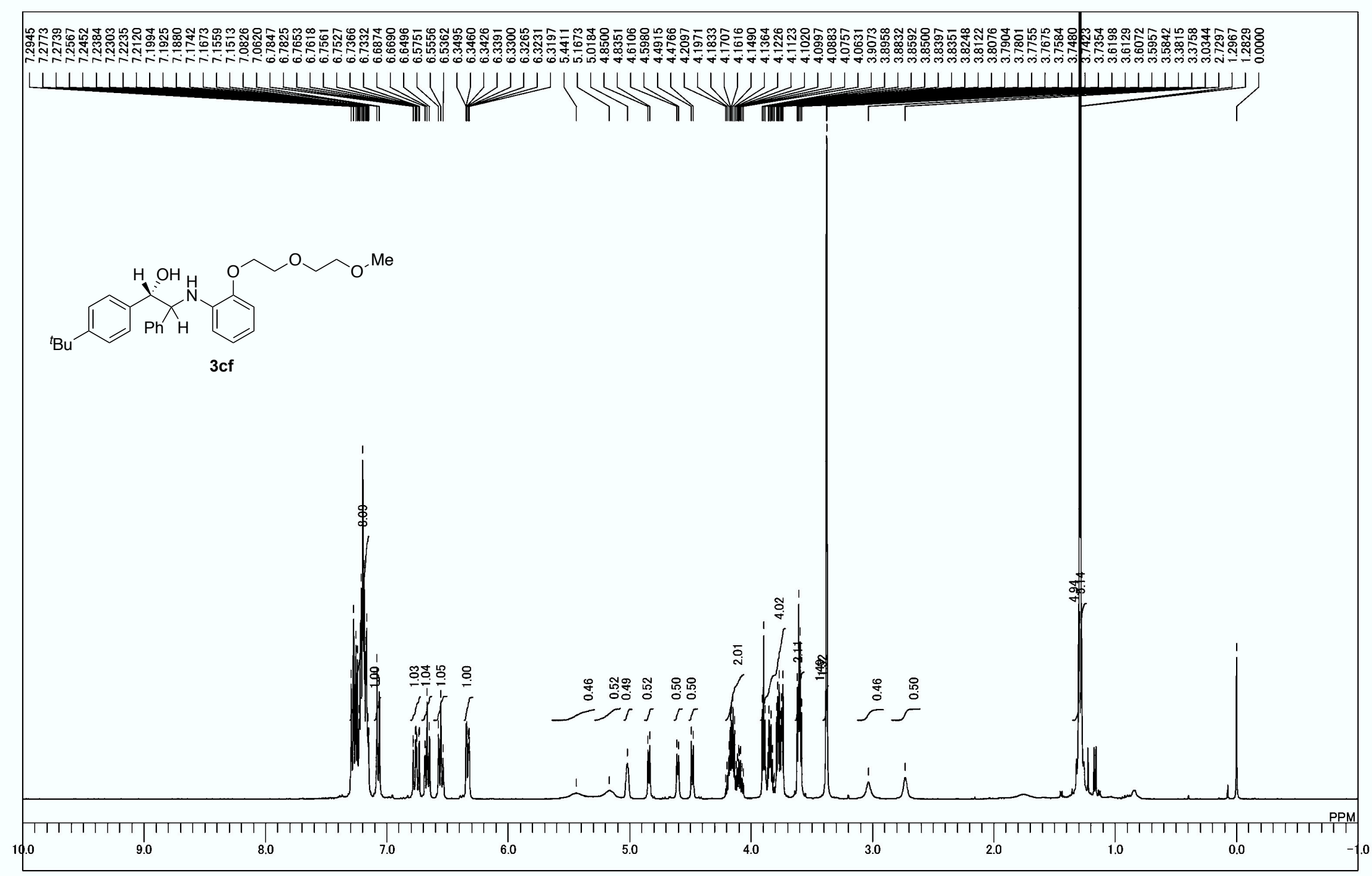

${ }^{1} \mathrm{H}$ NMR spectrum of $\mathbf{3 c f}$ 


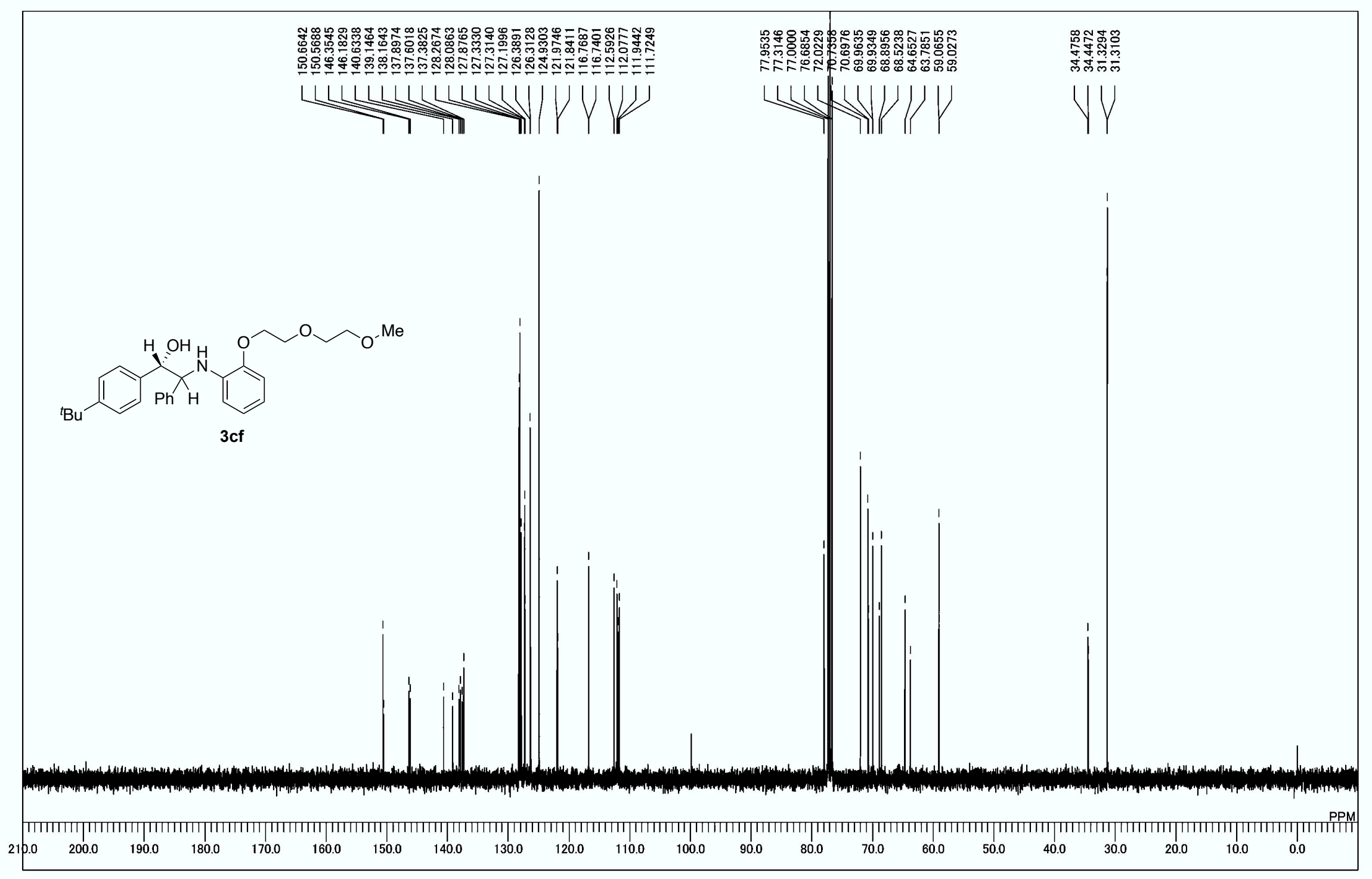

${ }^{13} \mathrm{C}$ NMR spectrum of $\mathbf{3 c f}$ 


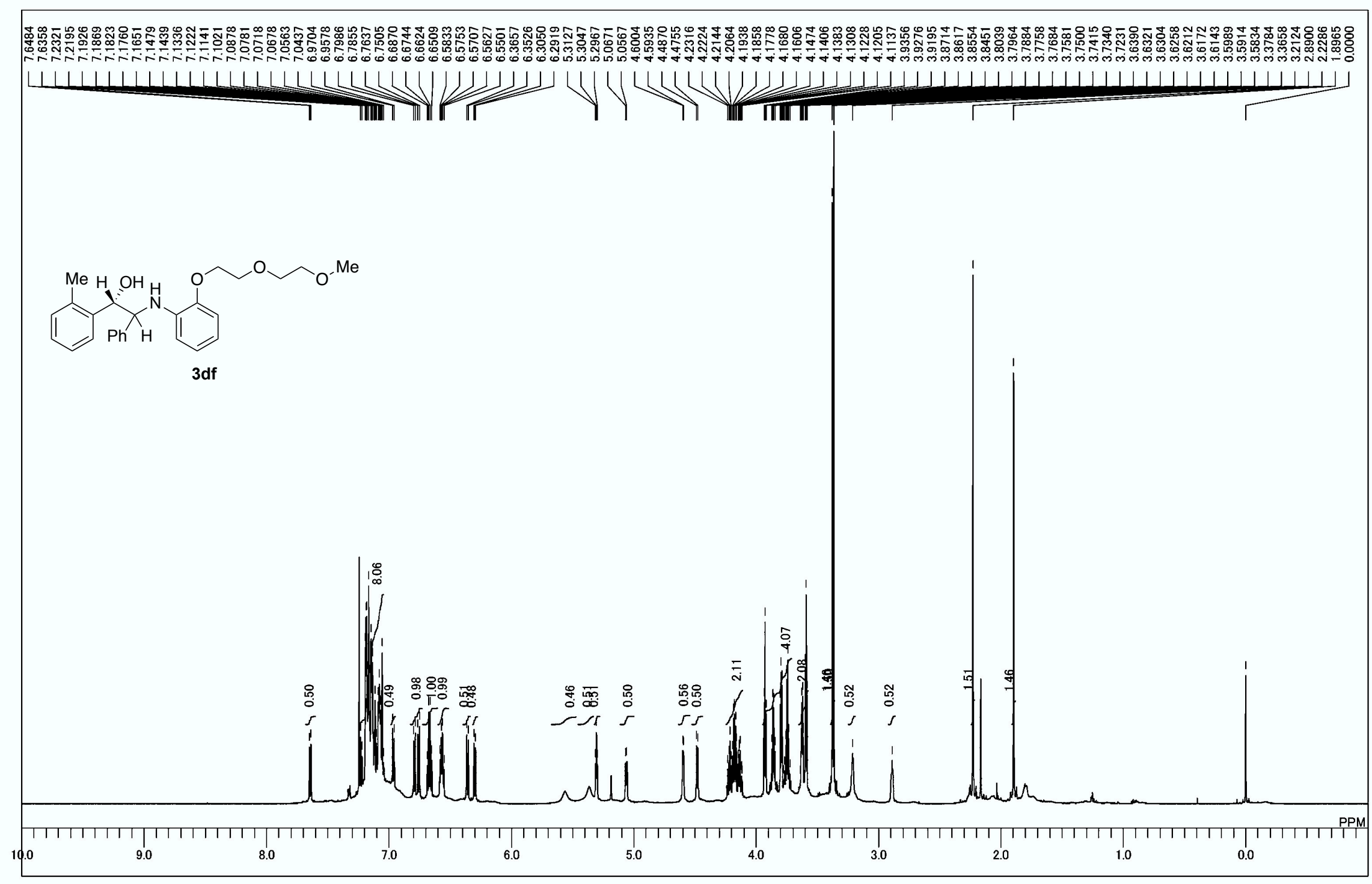

${ }^{1} \mathrm{H}$ NMR spectrum of $\mathbf{3 d f}$ 


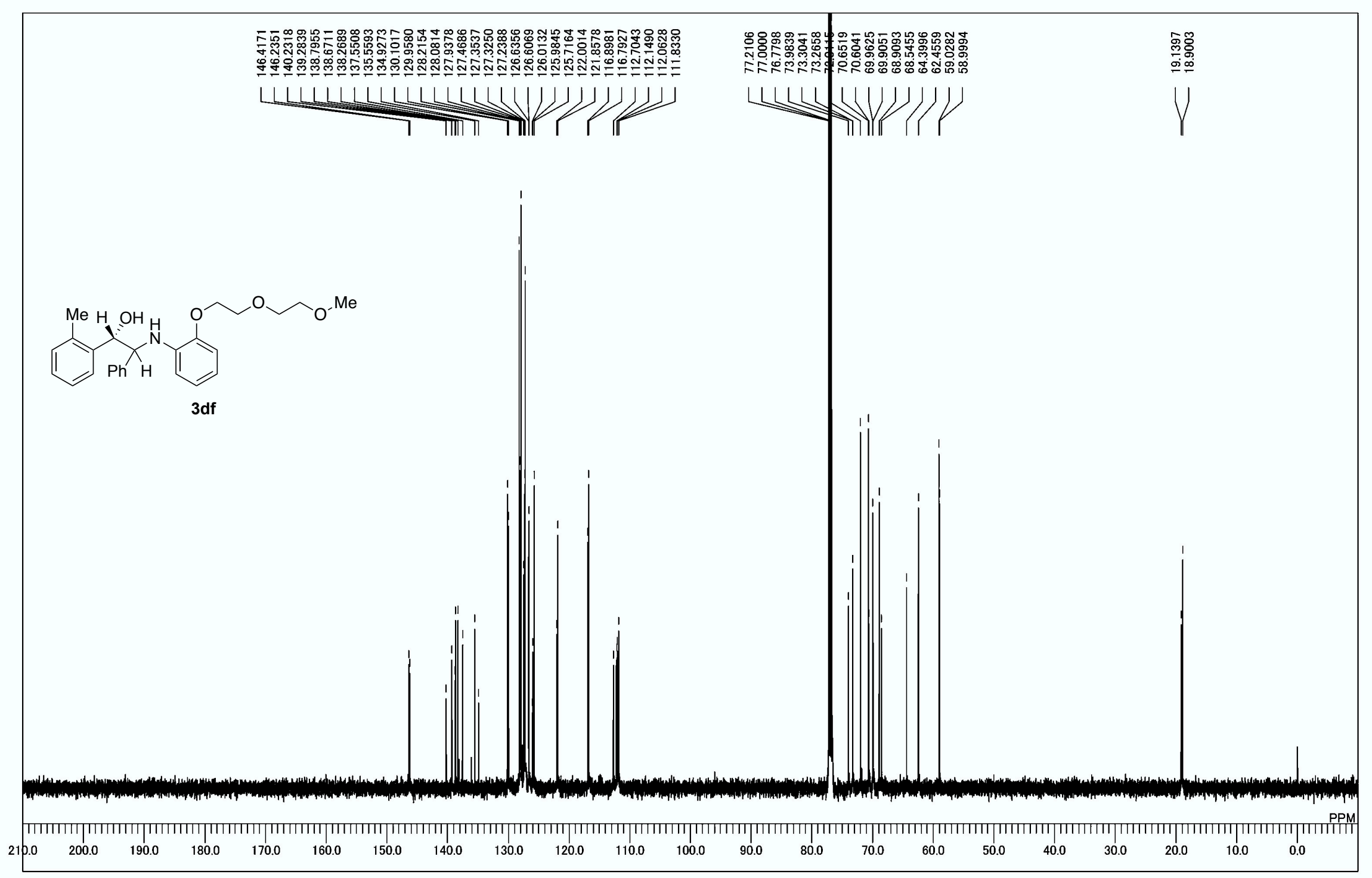

${ }^{13} \mathrm{C}$ NMR spectrum of $\mathbf{3 d f}$ 


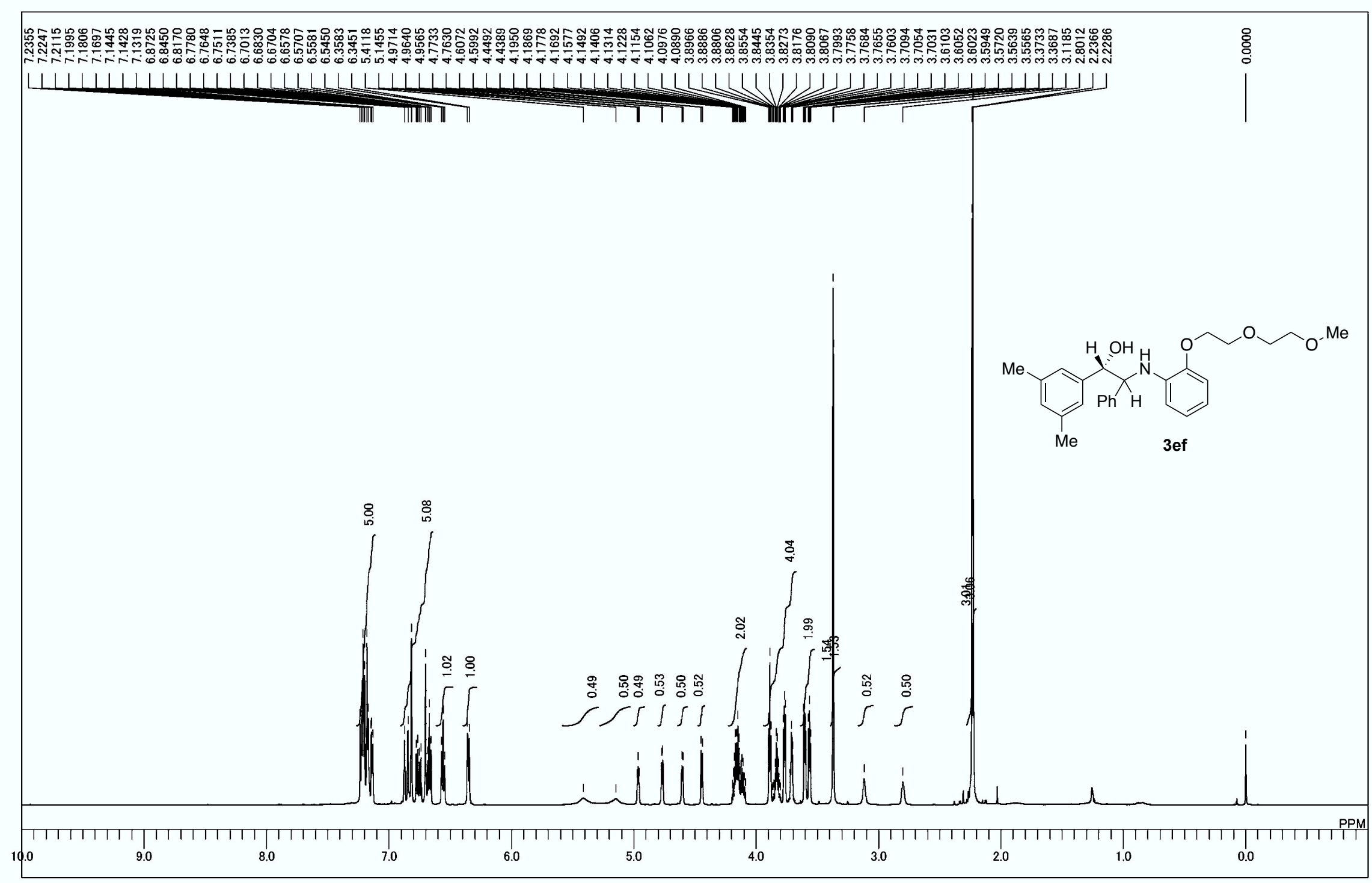

${ }^{1} \mathrm{H}$ NMR spectrum of $\mathbf{3 e f}$ 


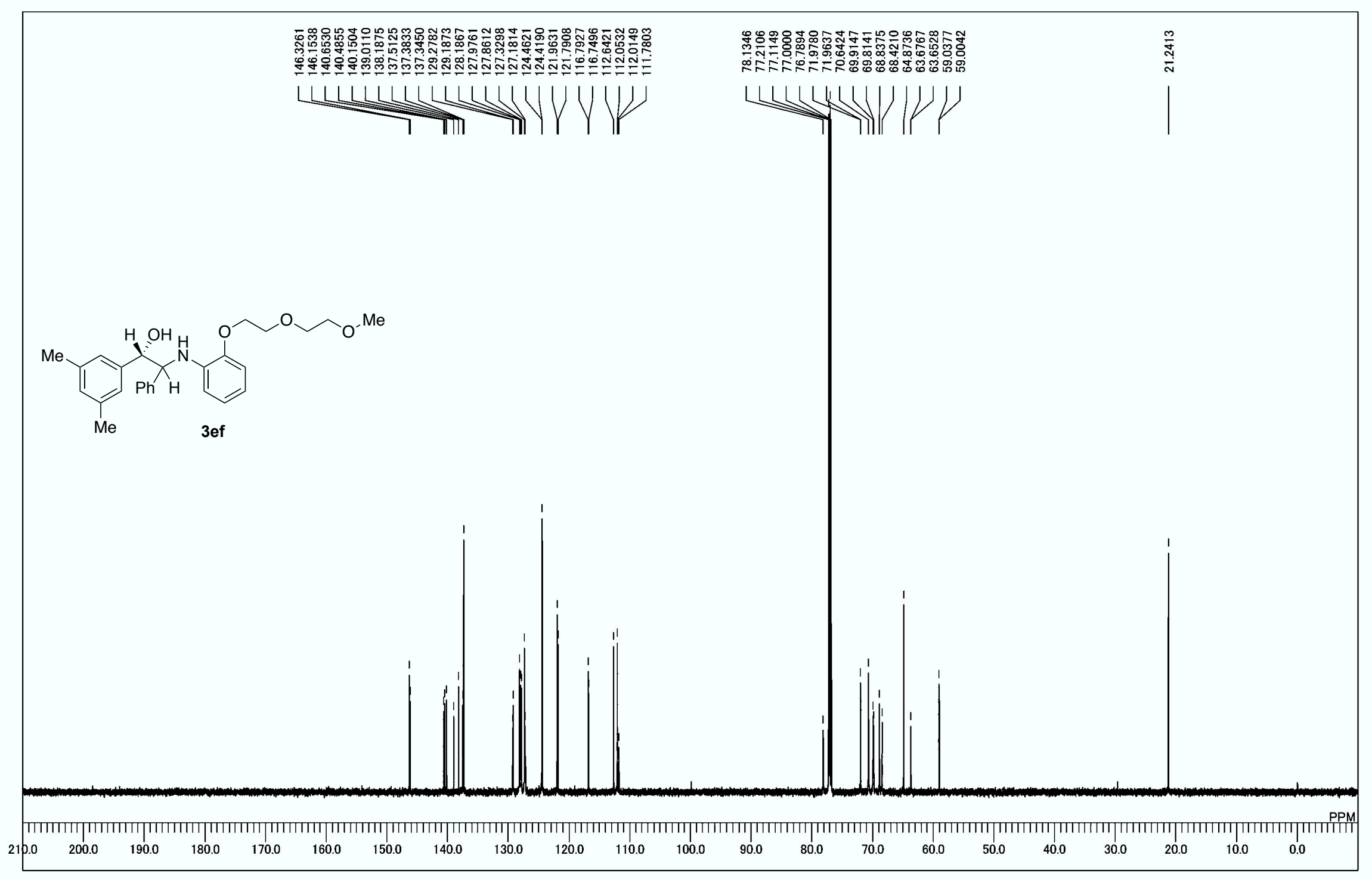

${ }^{13} \mathrm{C}$ NMR spectrum of $3 \mathbf{e f}$ 


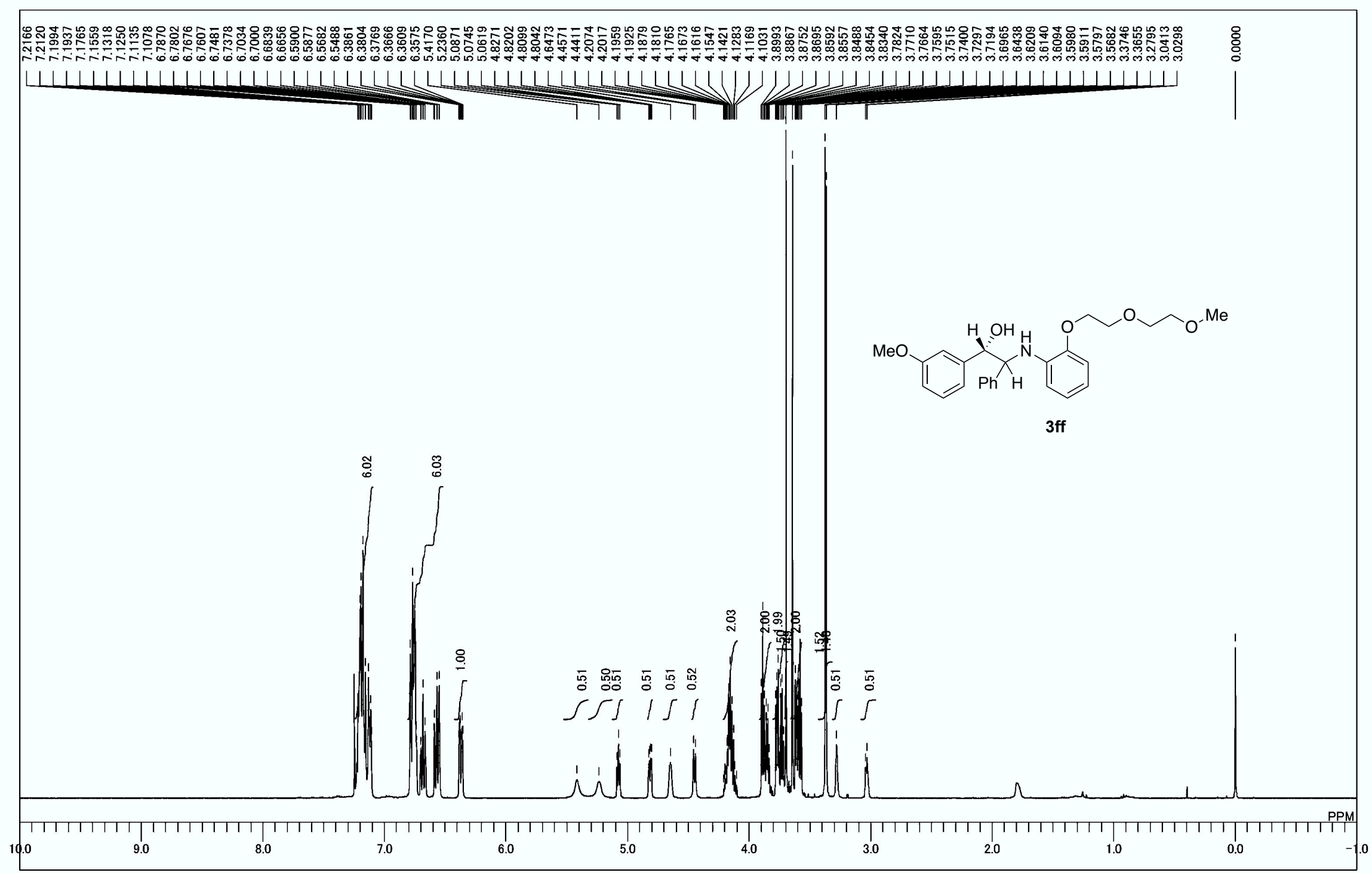

${ }^{1} \mathrm{H}$ NMR spectrum of $\mathbf{3 f f}$ 


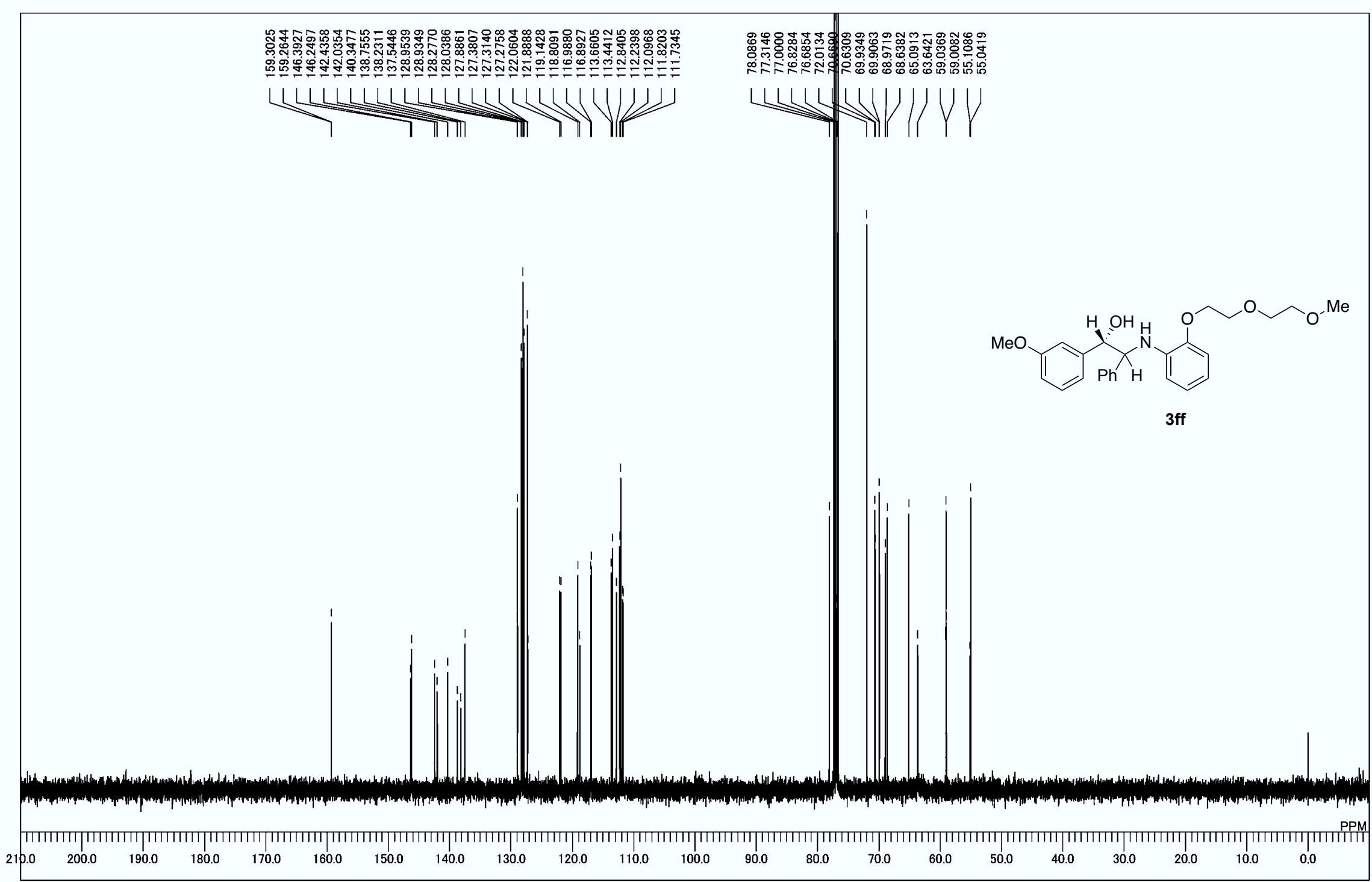

${ }^{13} \mathrm{C}$ NMR spectrum of $\mathbf{3 f f}$ 


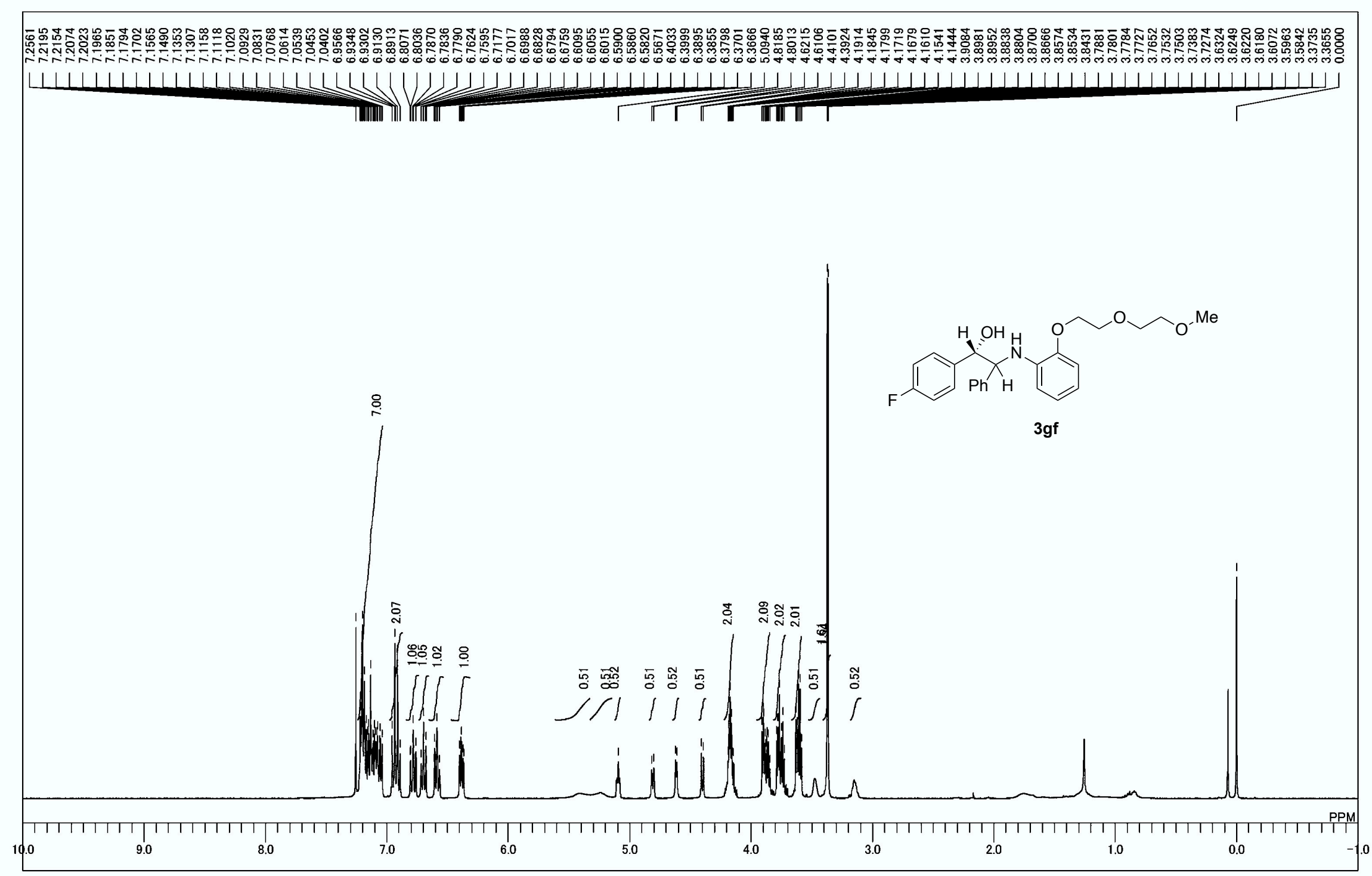

${ }^{1} \mathrm{H}$ NMR spectrum of $\mathbf{3 g f}$ 


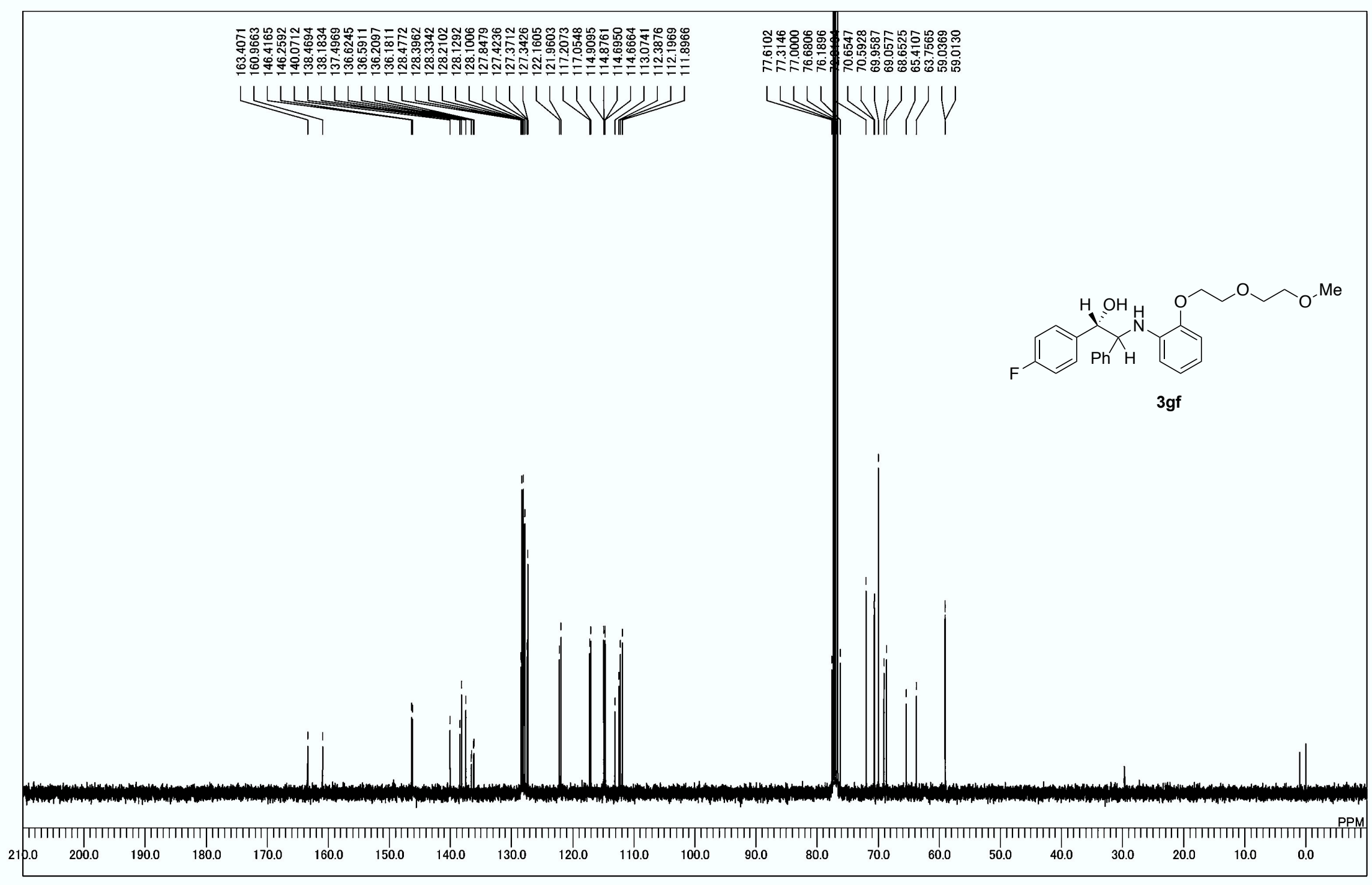

${ }^{13} \mathrm{C}$ NMR spectrum of $\mathbf{3 g f}$ 


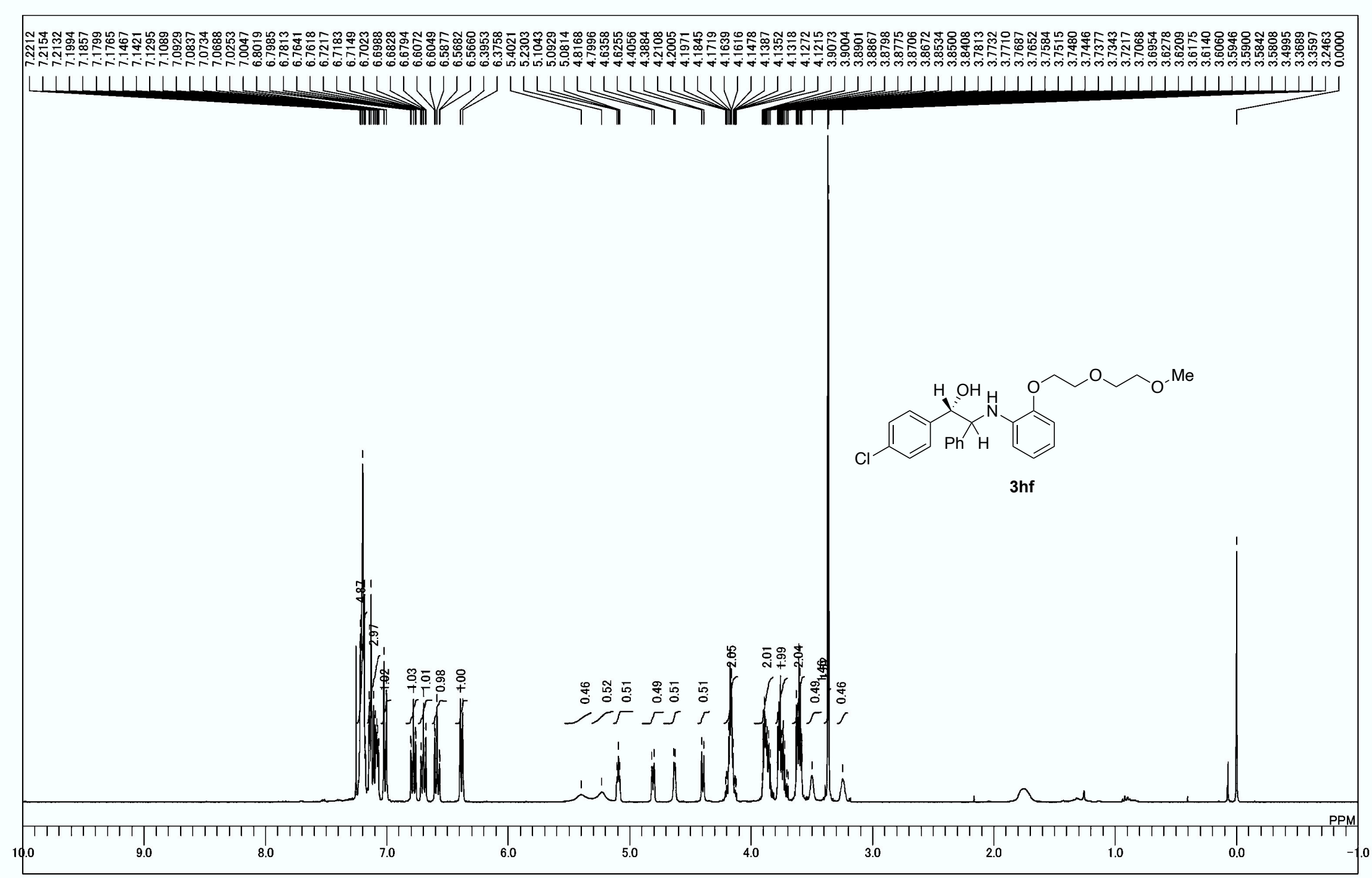

${ }^{1} \mathrm{H}$ NMR spectrum of $\mathbf{3 h f}$ 


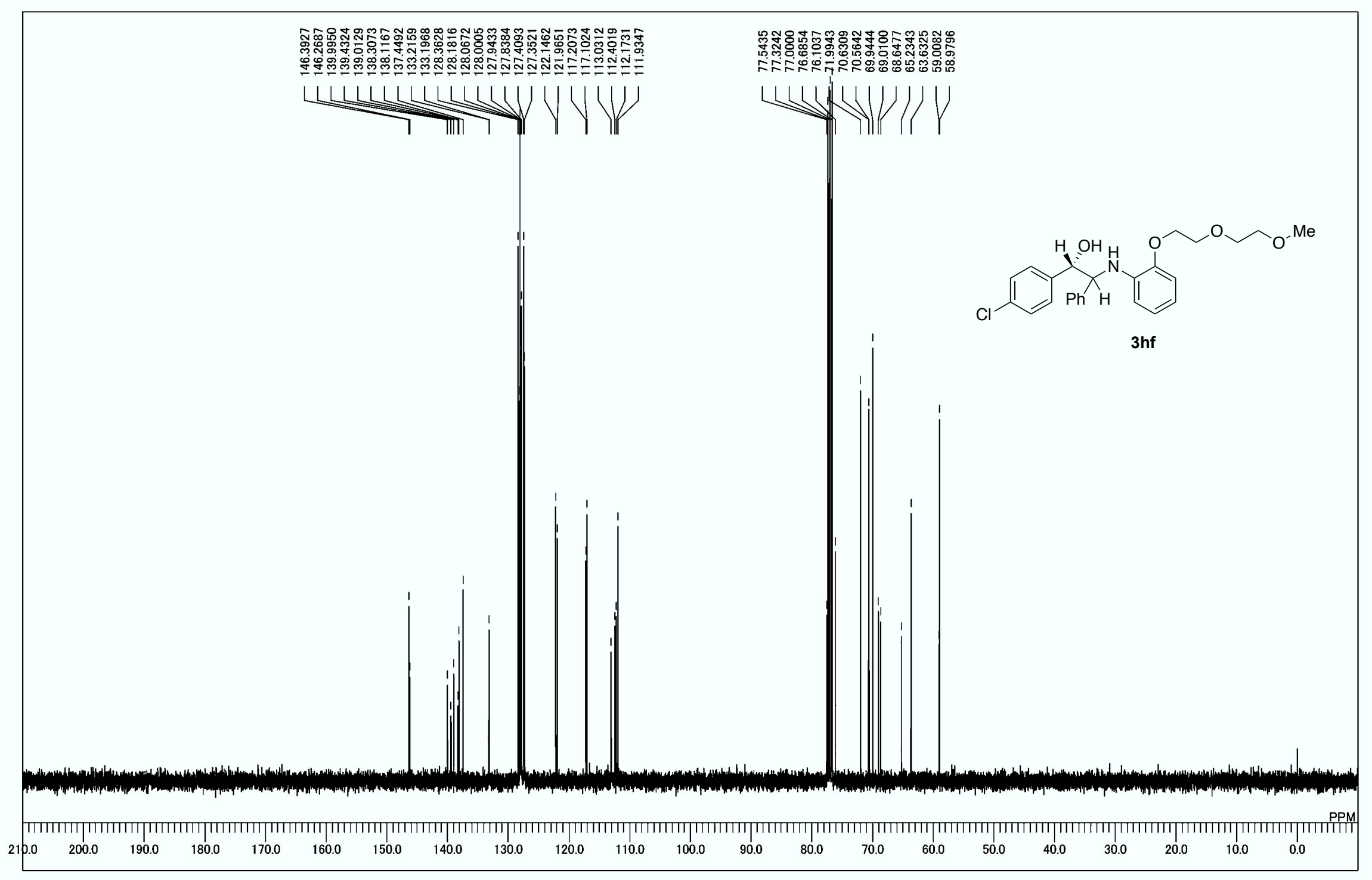

${ }^{13} \mathrm{C}$ NMR spectrum of $\mathbf{3 h f}$ 


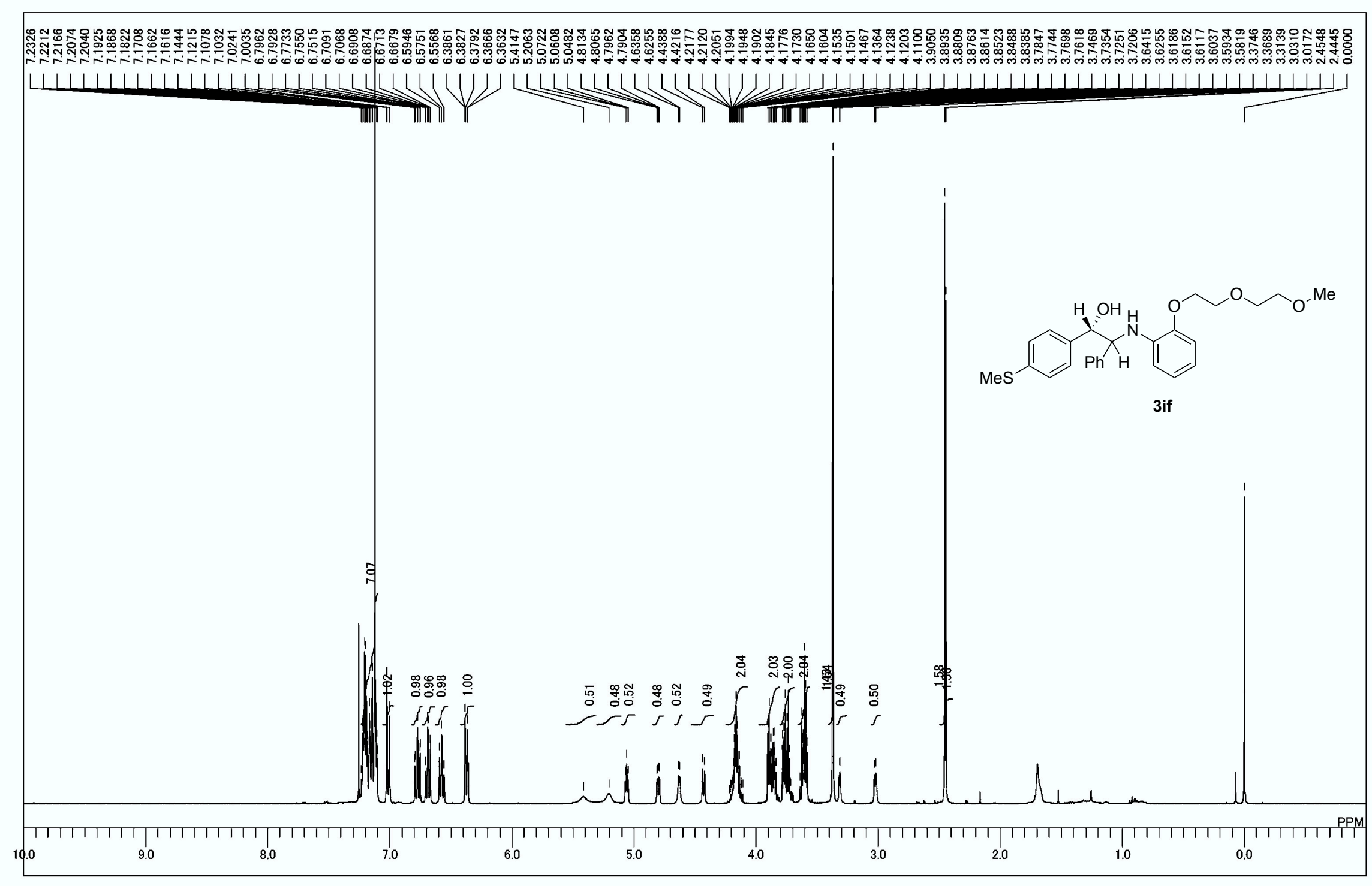

${ }^{1} \mathrm{H}$ NMR spectrum of 3if 


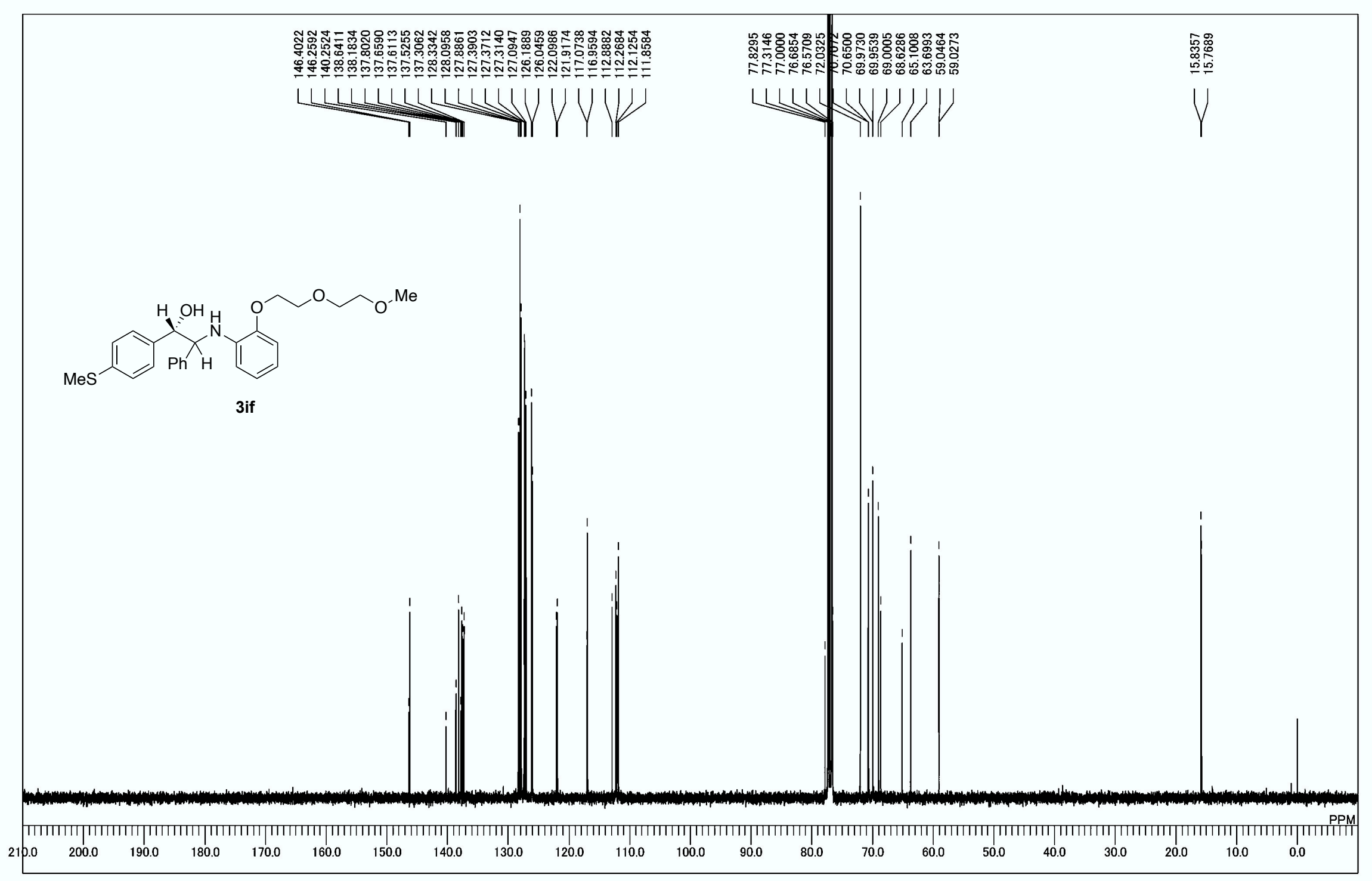

${ }^{13} \mathrm{C}$ NMR spectrum of $\mathbf{3 i f}$ 


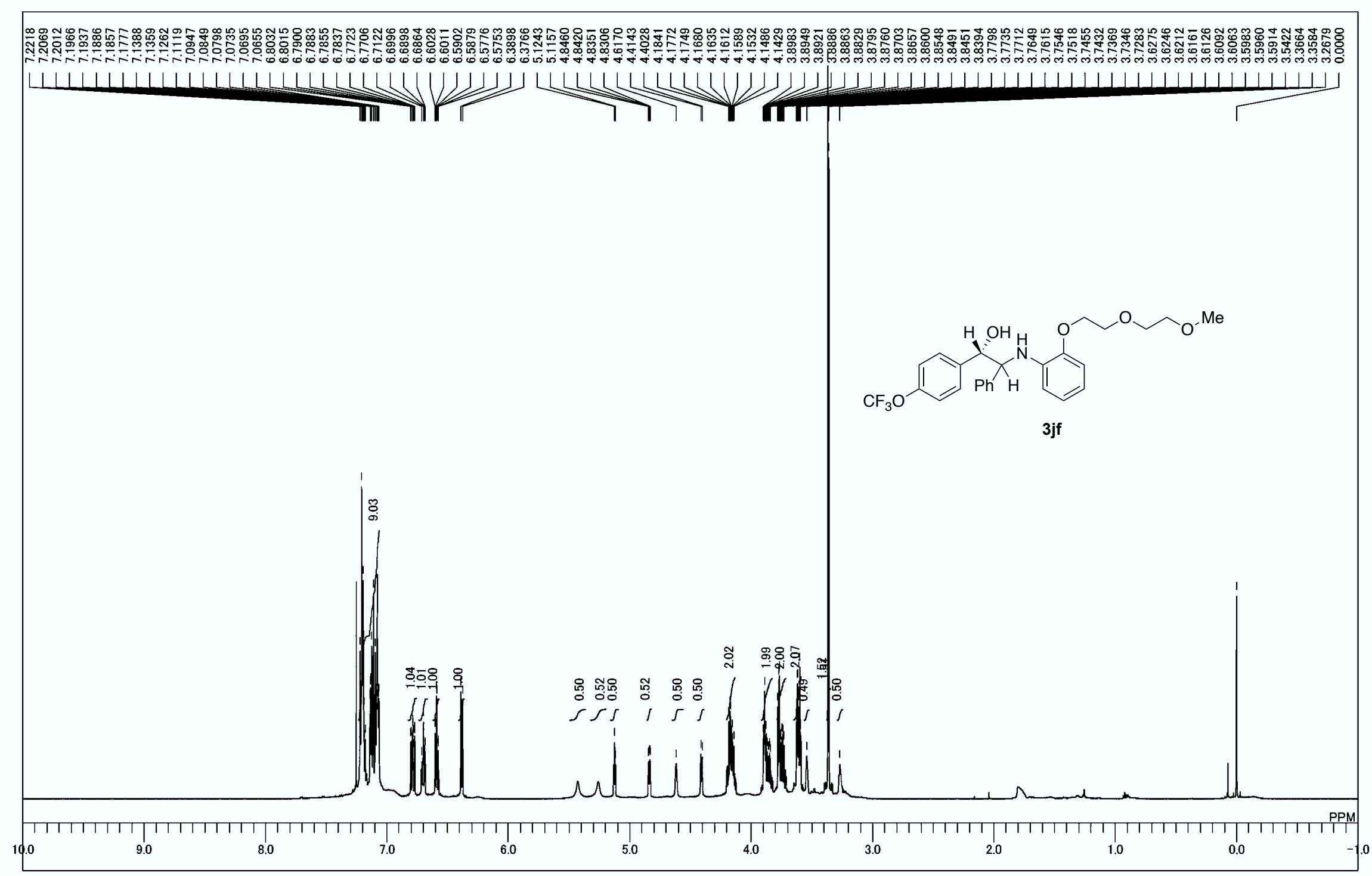

${ }^{1}$ H NMR spectrum of $\mathbf{3 j f}$ 


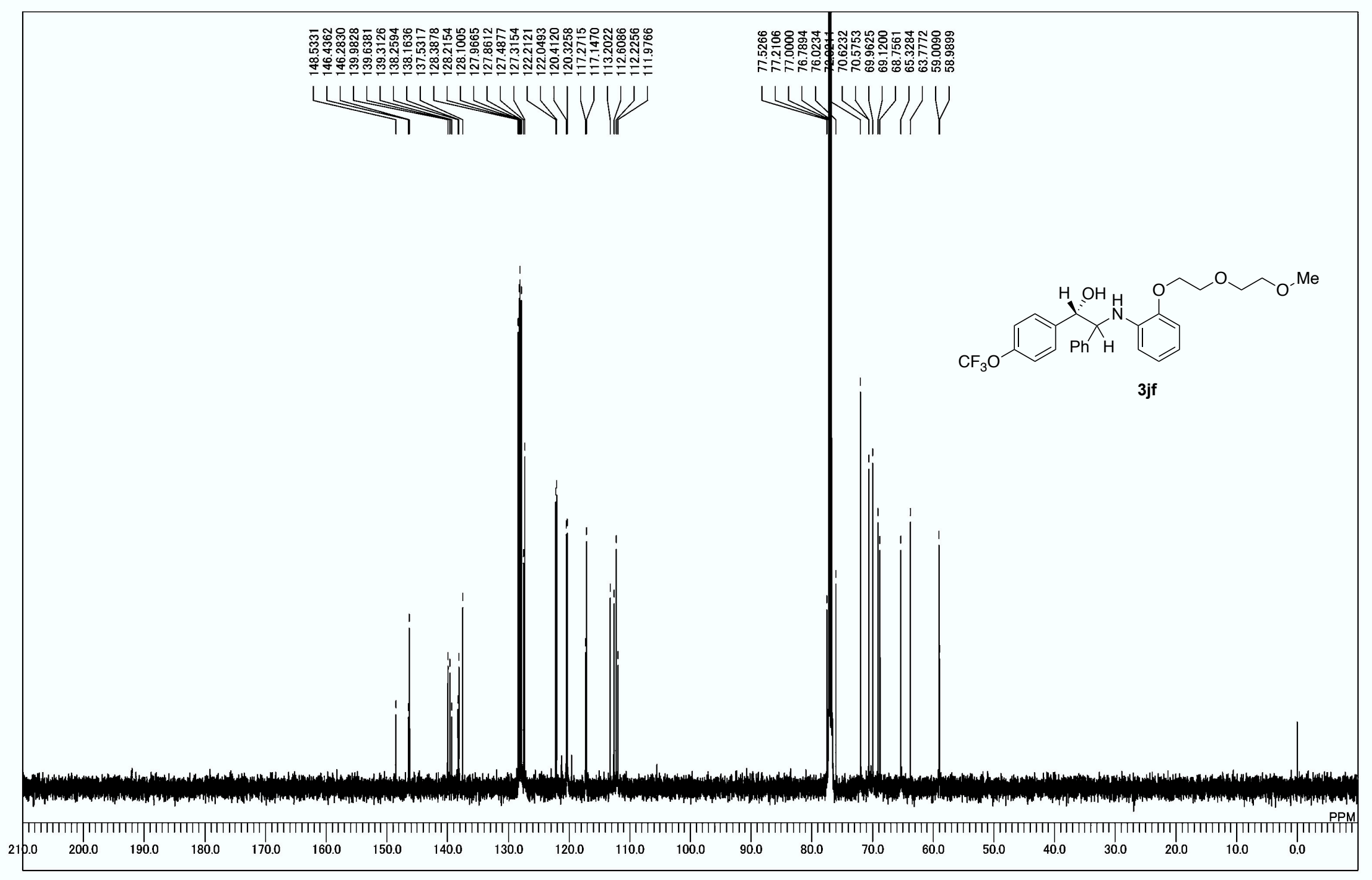

${ }^{13} \mathrm{C}$ NMR spectrum of $\mathbf{3 j \mathbf { f }}$ 


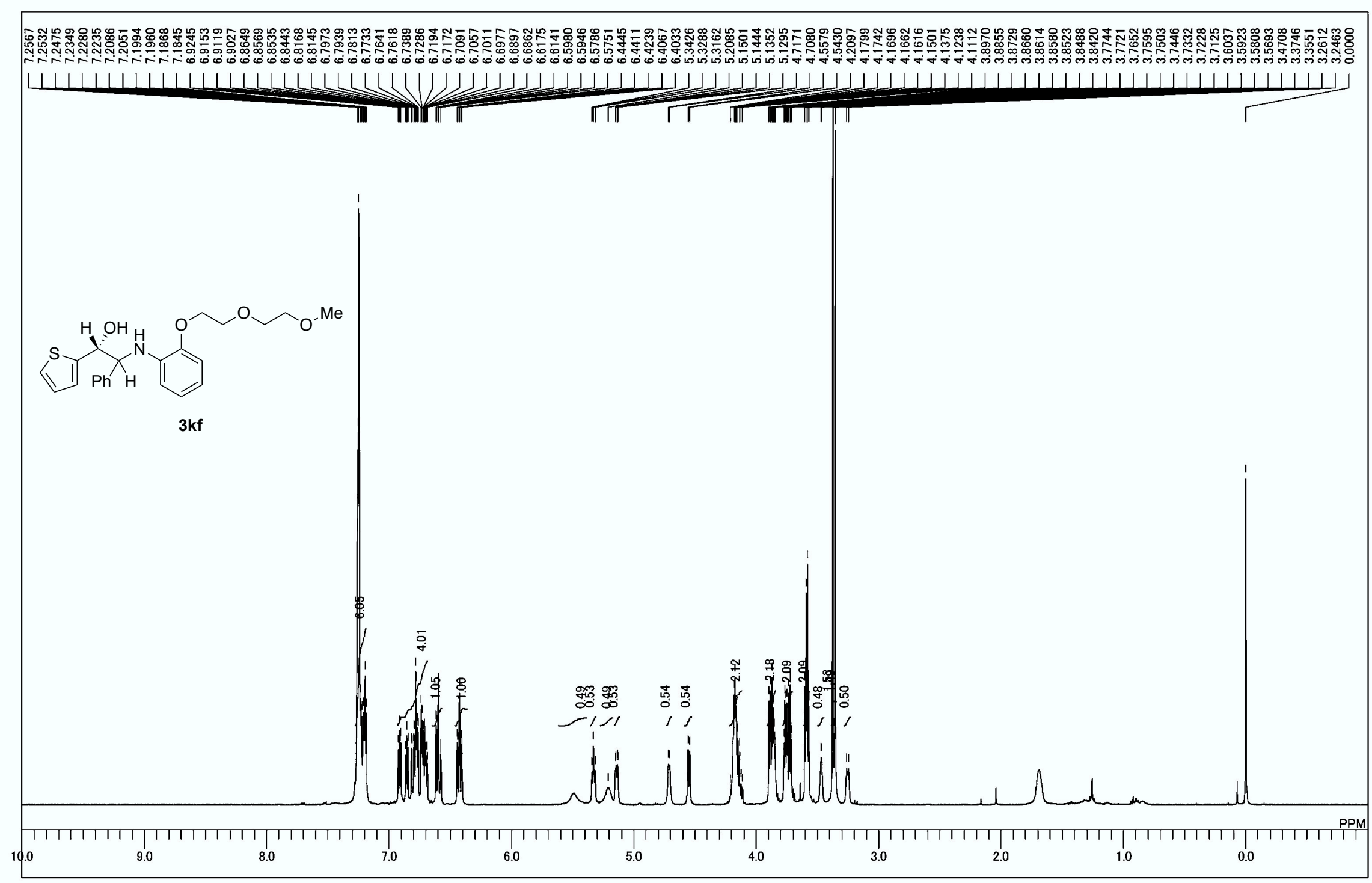

${ }^{1} \mathrm{H}$ NMR spectrum of $\mathbf{3 k f}$ 


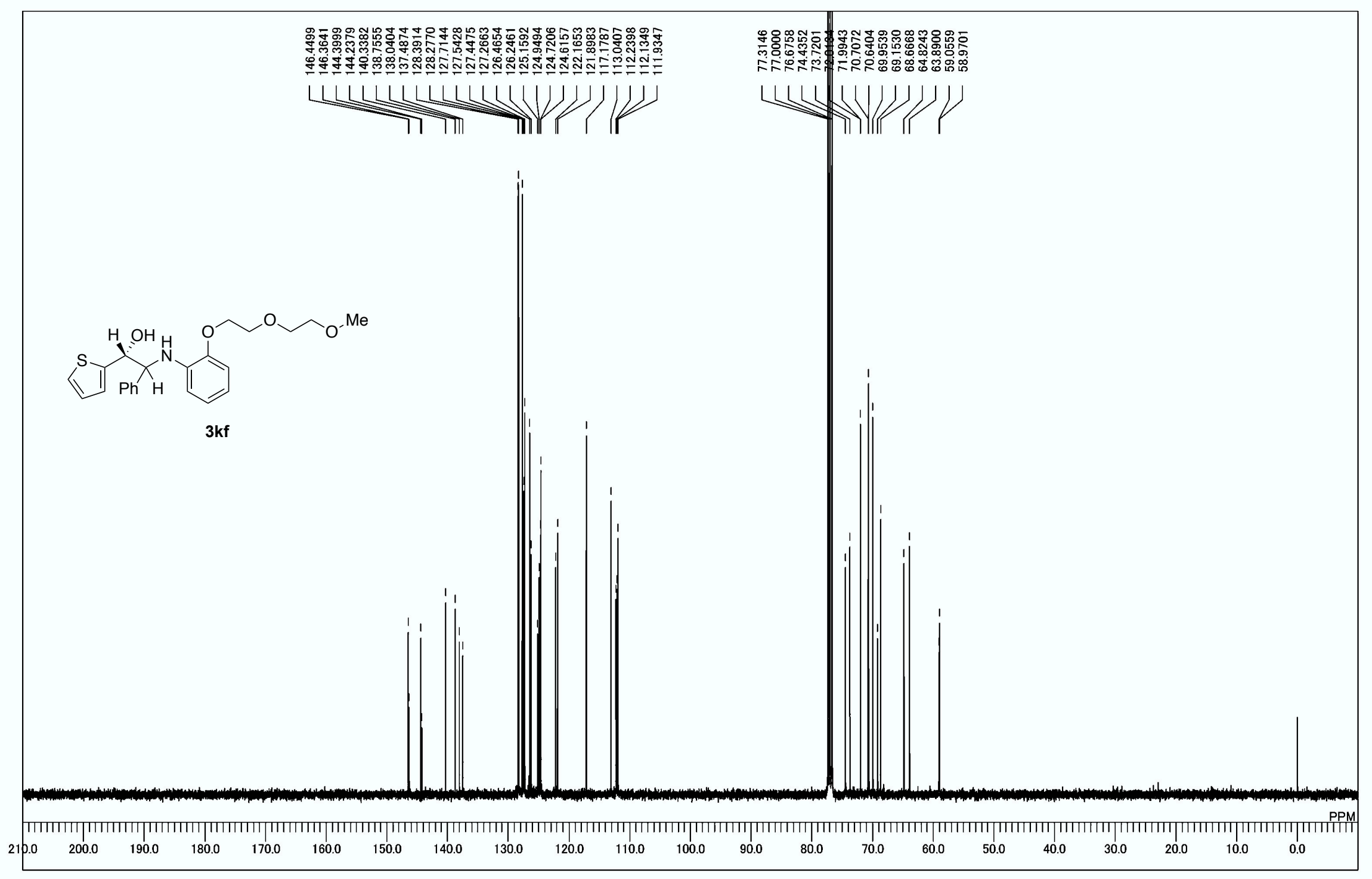

${ }^{13} \mathrm{C}$ NMR spectrum of $\mathbf{3 k f}$ 


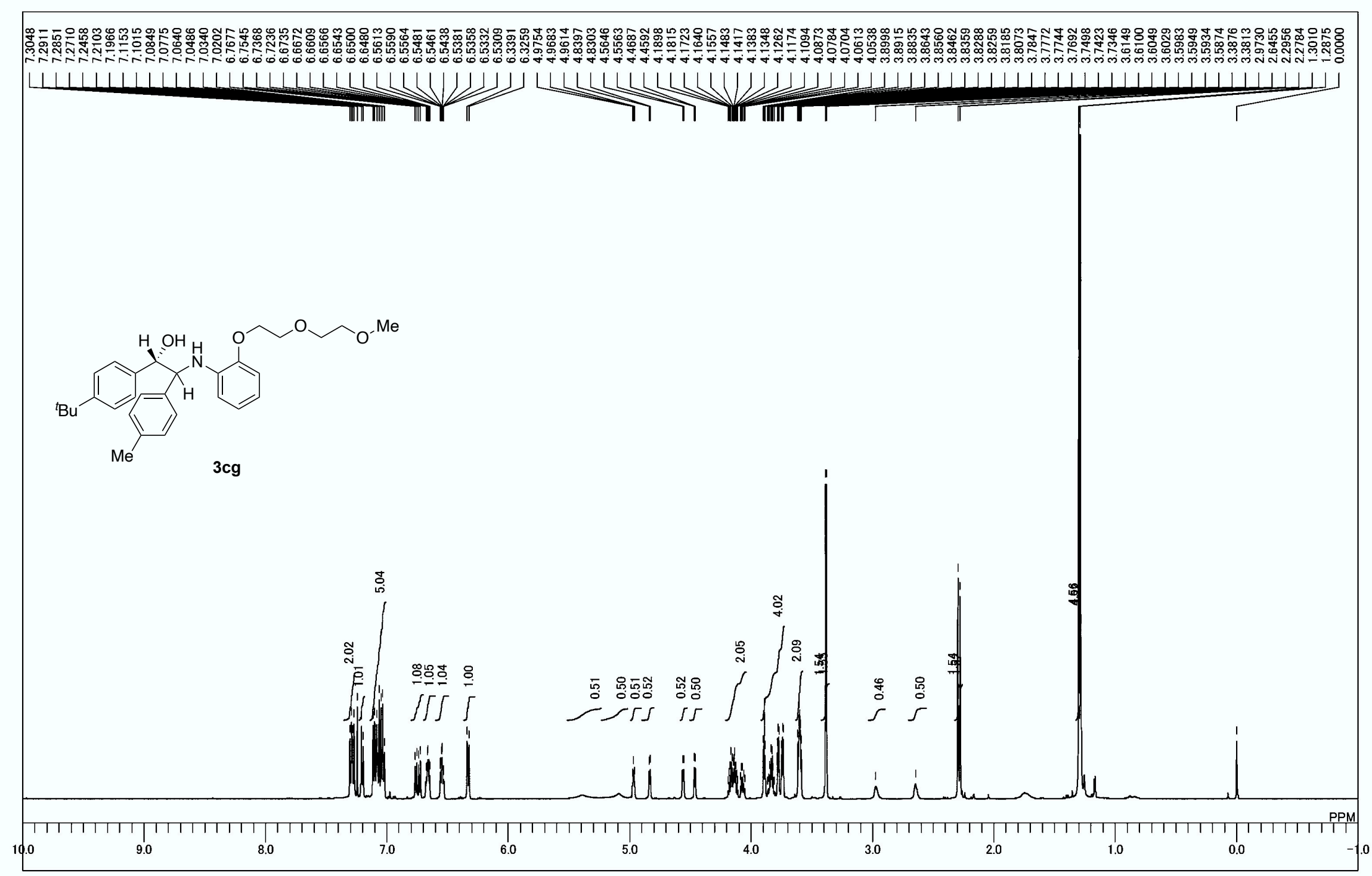

${ }^{1} \mathrm{H}$ NMR spectrum of $\mathbf{3 c g}$ 


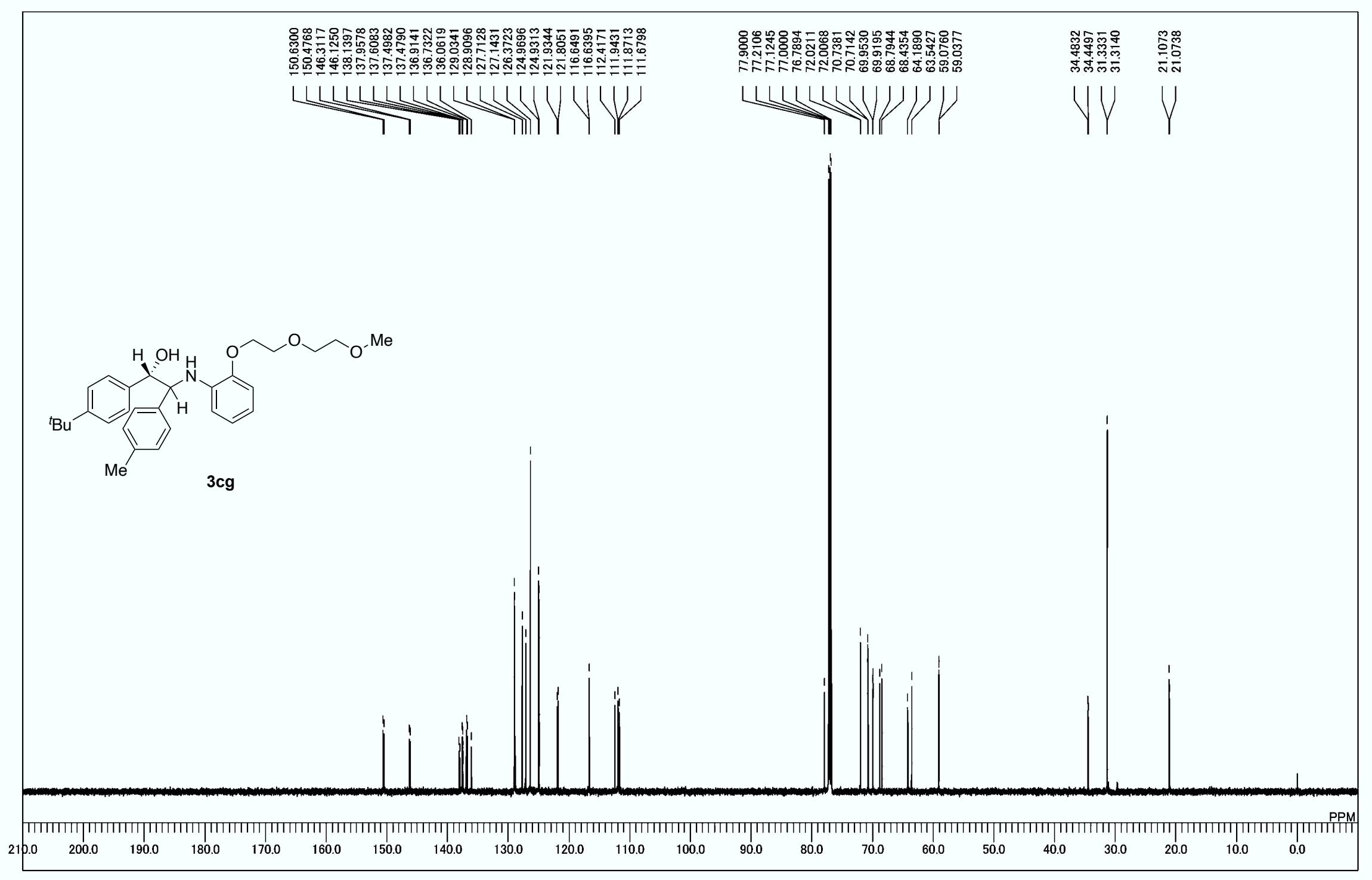

${ }^{13} \mathrm{C}$ NMR spectrum of $\mathbf{3} \mathrm{cg}$ 


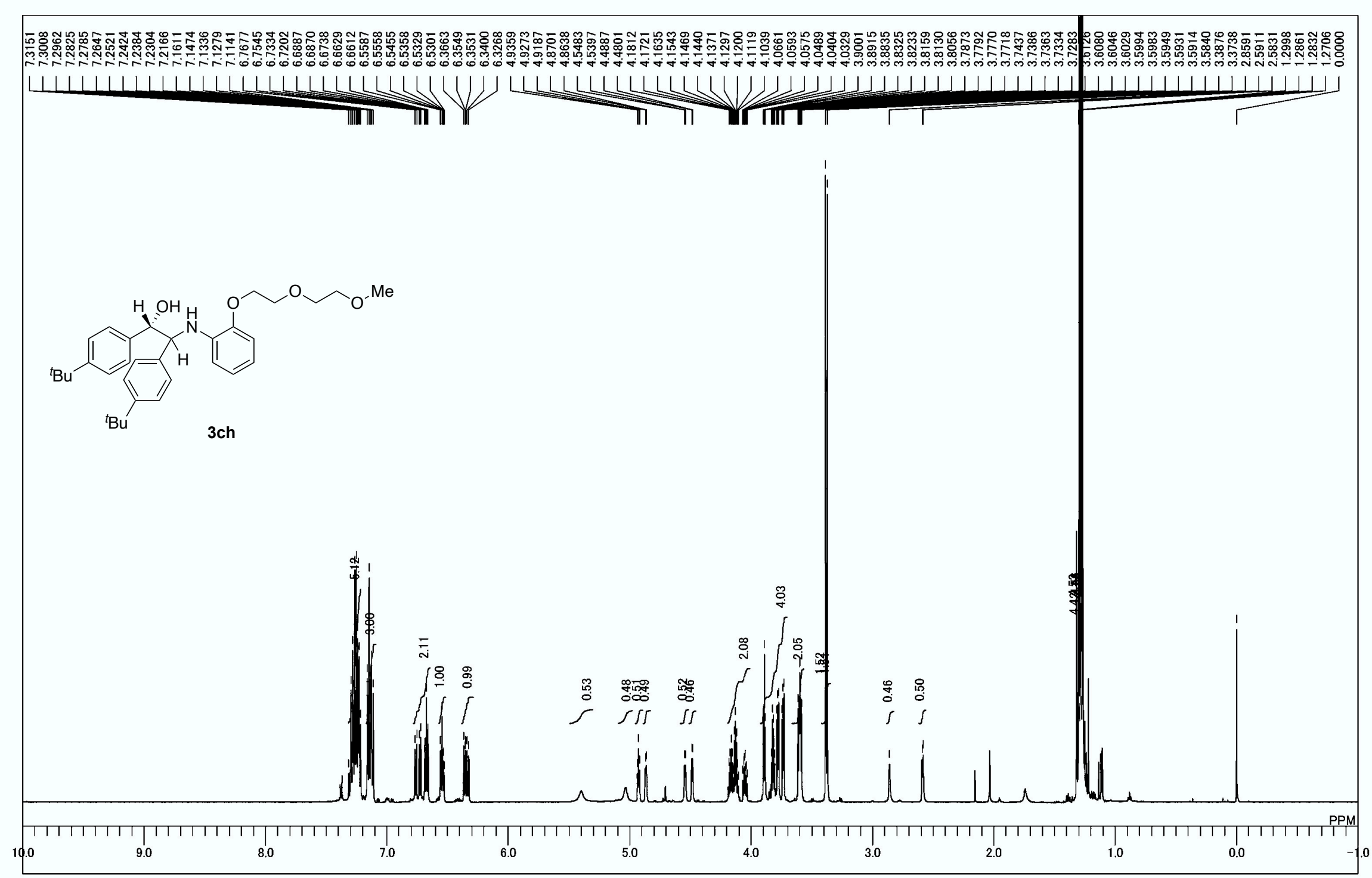

${ }^{1} \mathrm{H}$ NMR spectrum of $\mathbf{3} \mathbf{c h}$ 


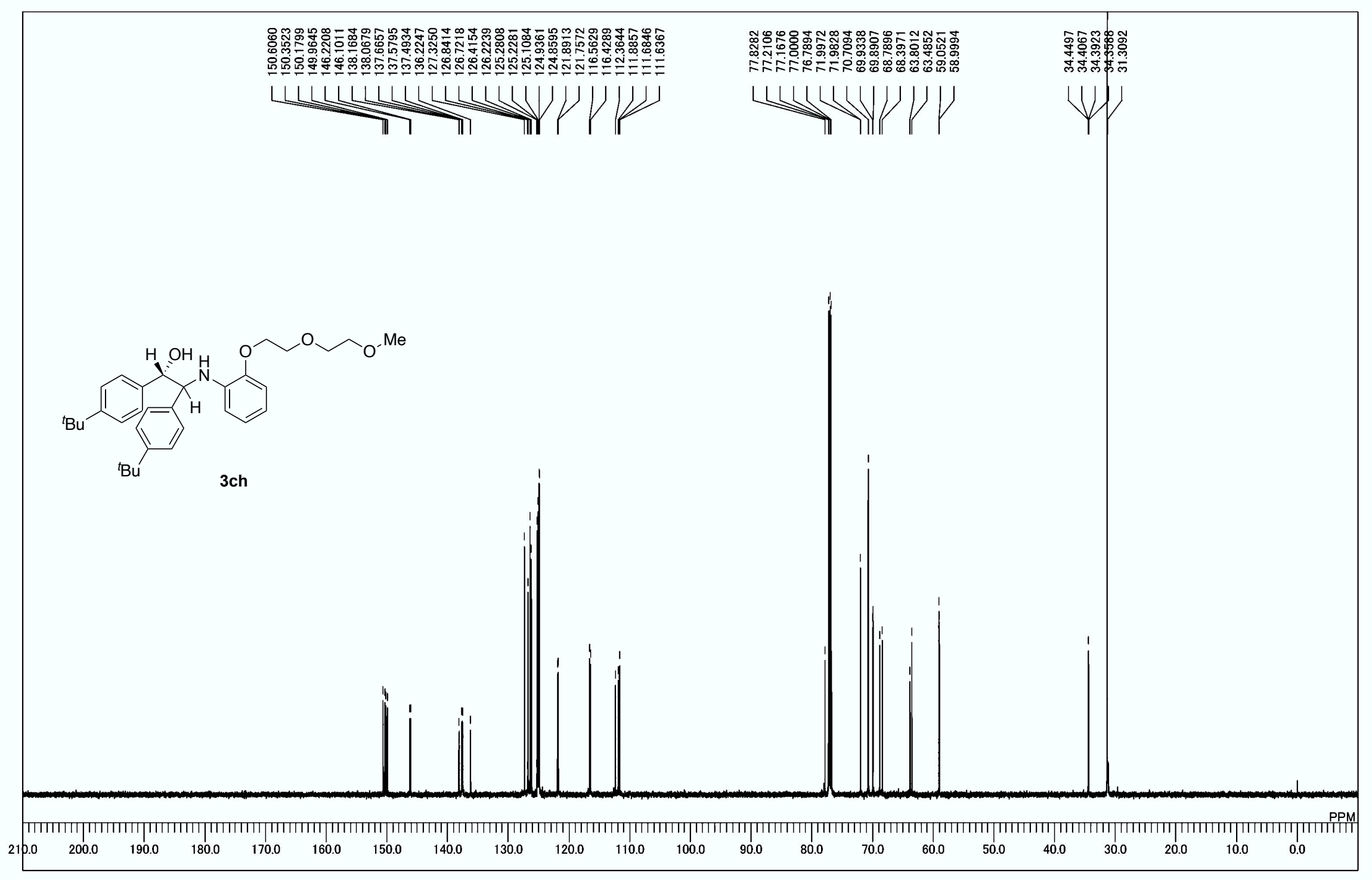

${ }^{13} \mathrm{C}$ NMR spectrum of $\mathbf{3 c h}$ 


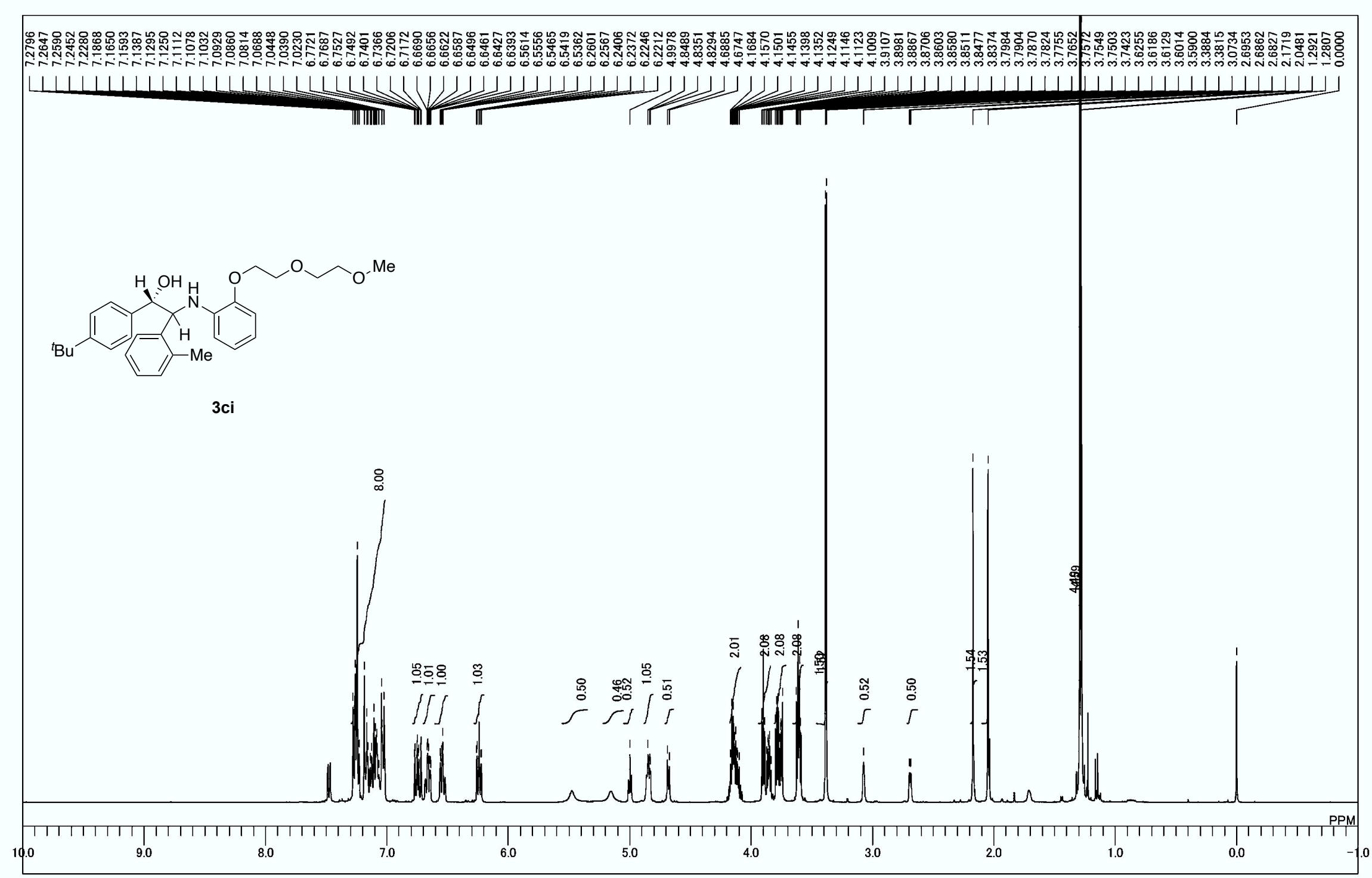

${ }^{1} \mathrm{H}$ NMR spectrum of $\mathbf{3 c i}$ 


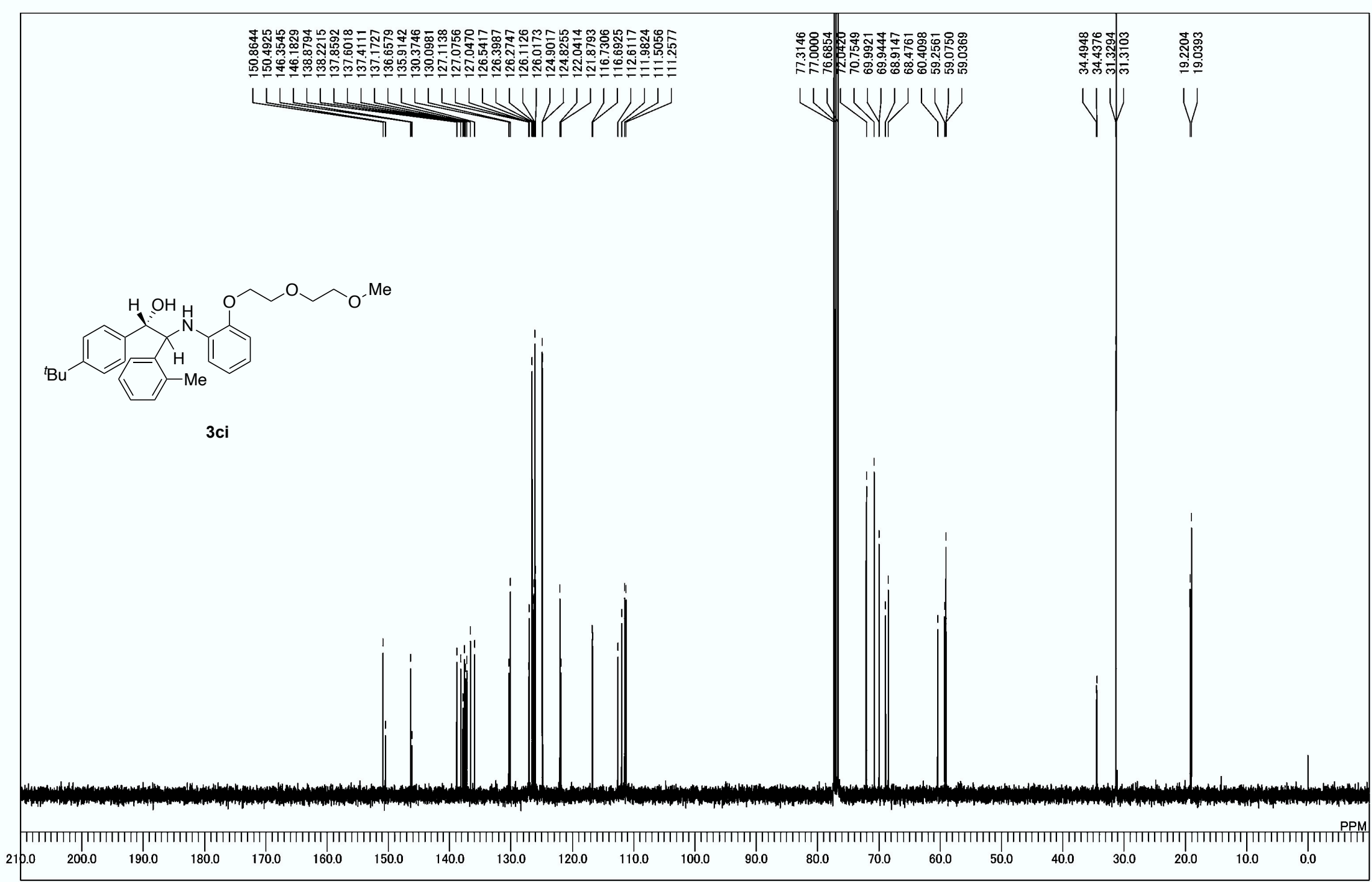

${ }^{13} \mathrm{C}$ NMR spectrum of $\mathbf{3 c i}$ 


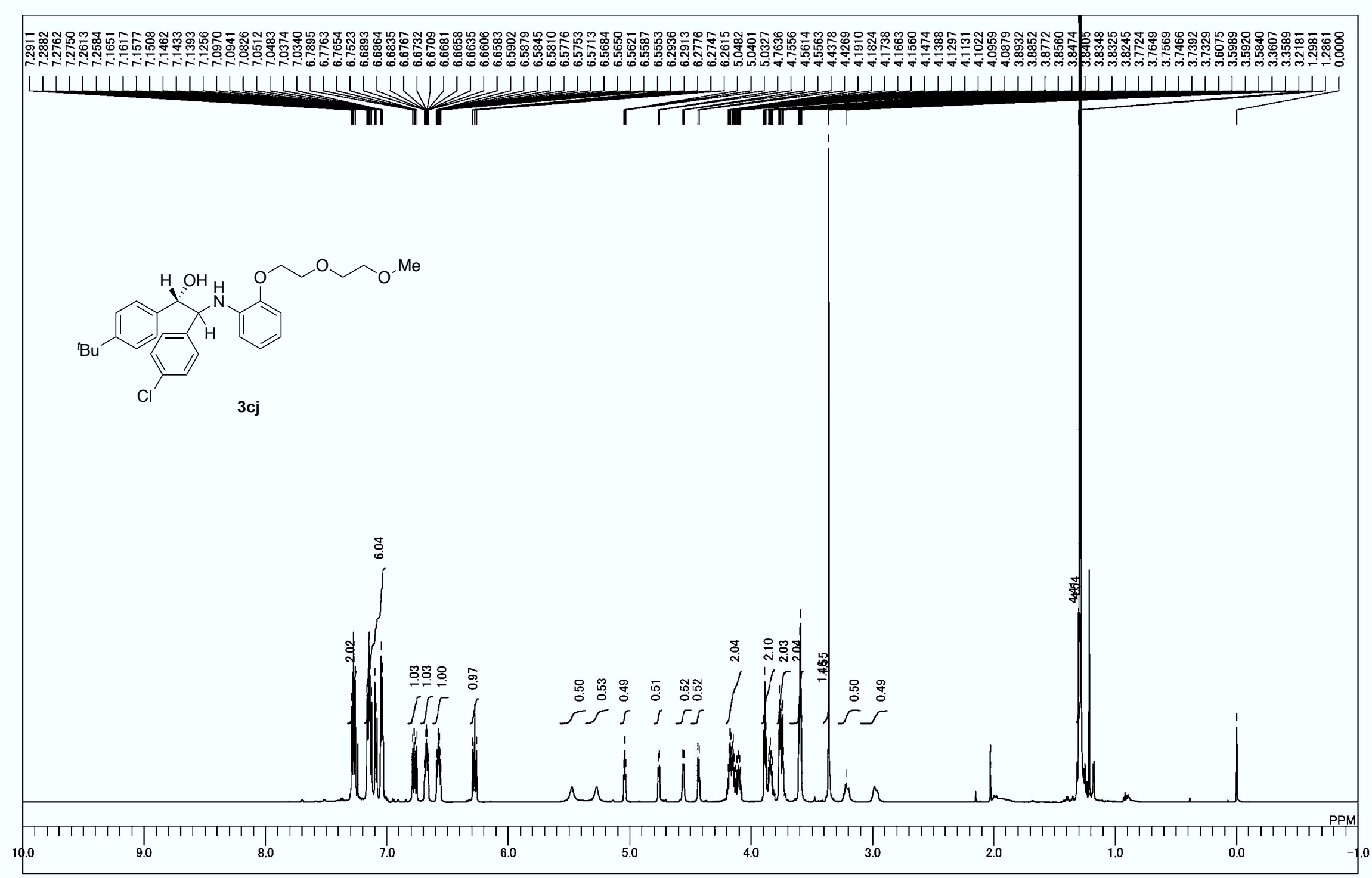

${ }^{1} \mathrm{H}$ NMR spectrum of $\mathbf{3 c j}$ 


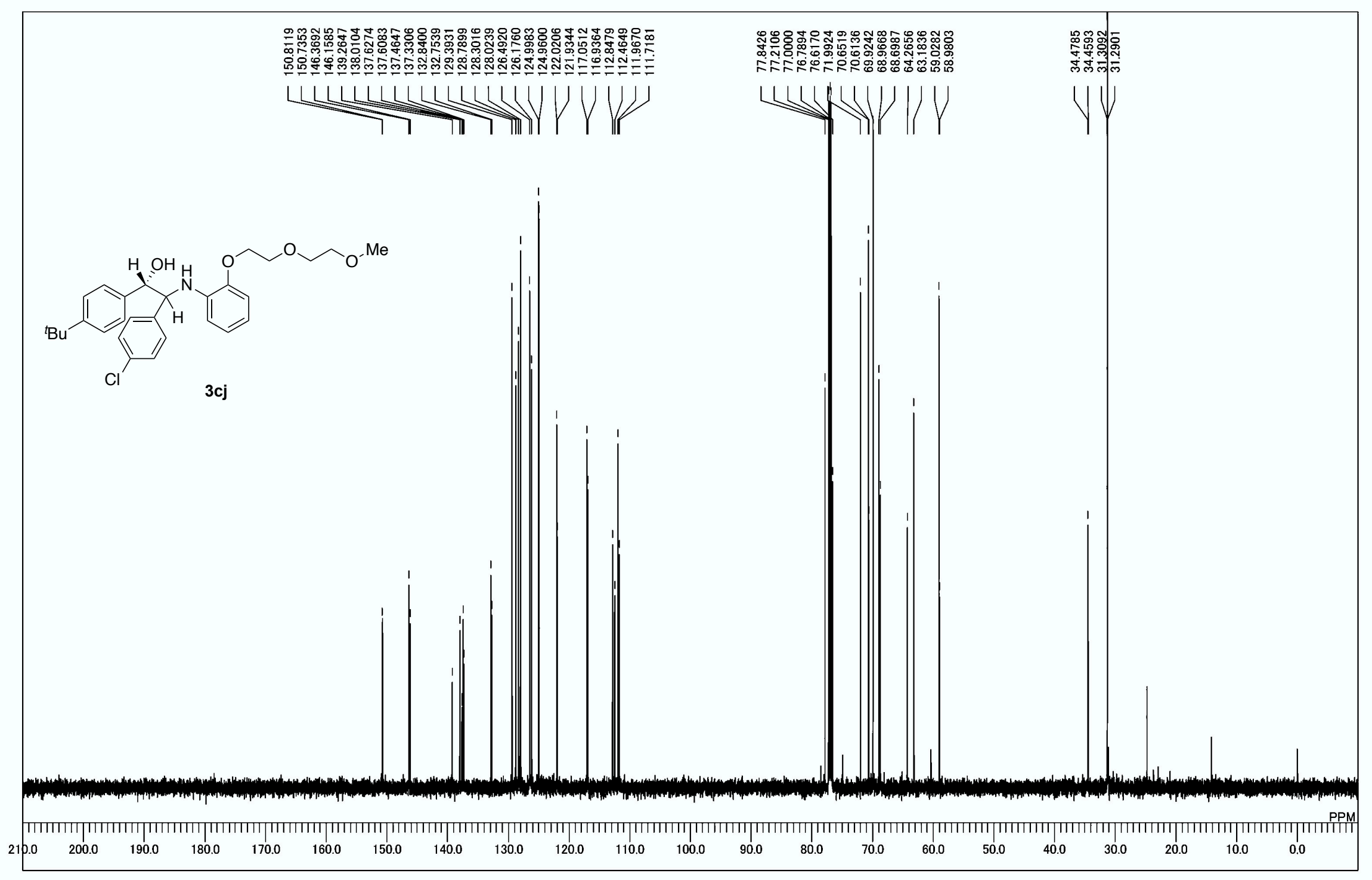

${ }^{13} \mathrm{C}$ NMR spectrum of $\mathbf{3} \mathbf{c j}$ 


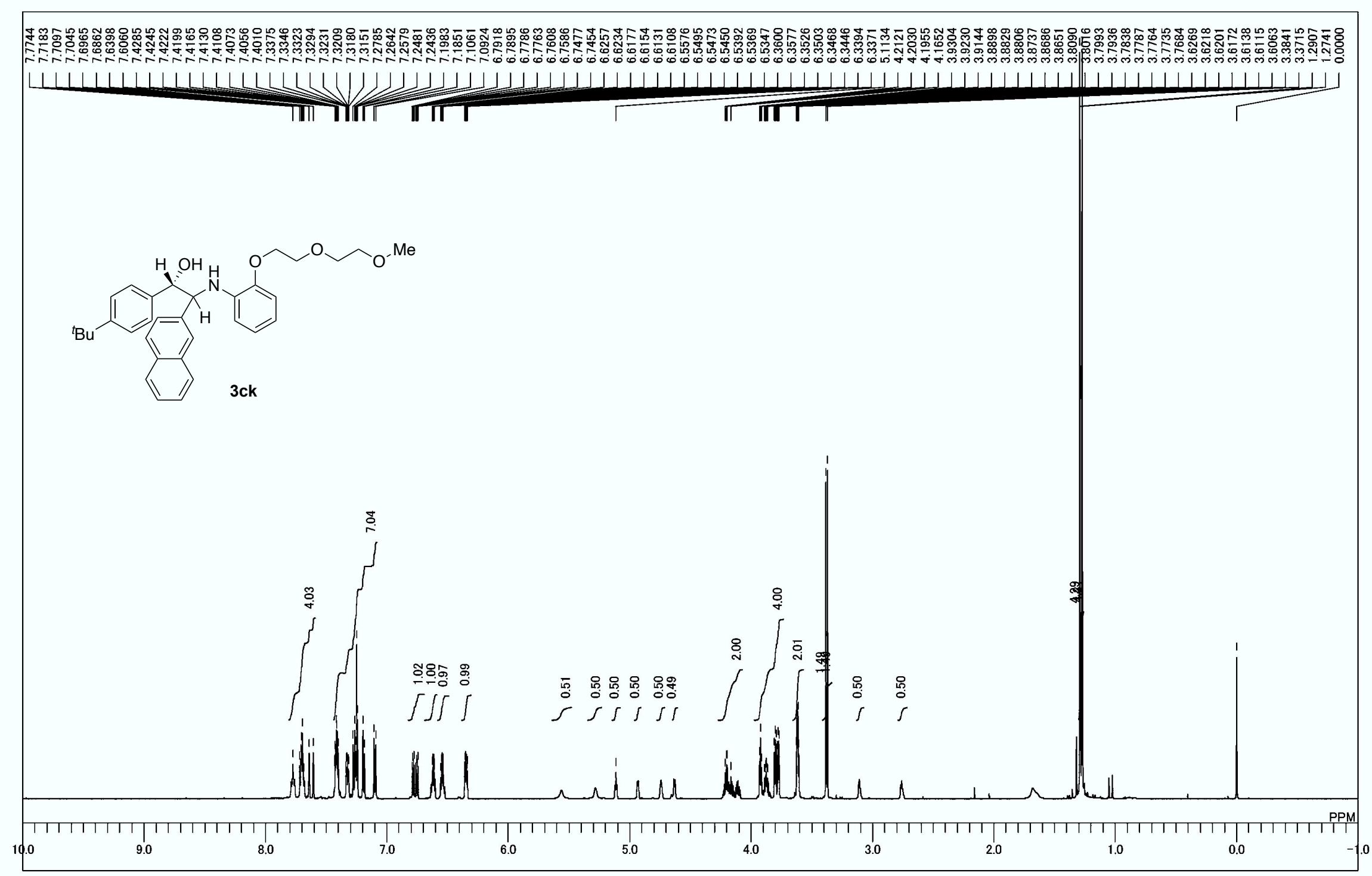

${ }^{1} \mathrm{H}$ NMR spectrum of $\mathbf{3} \mathbf{c k}$ 


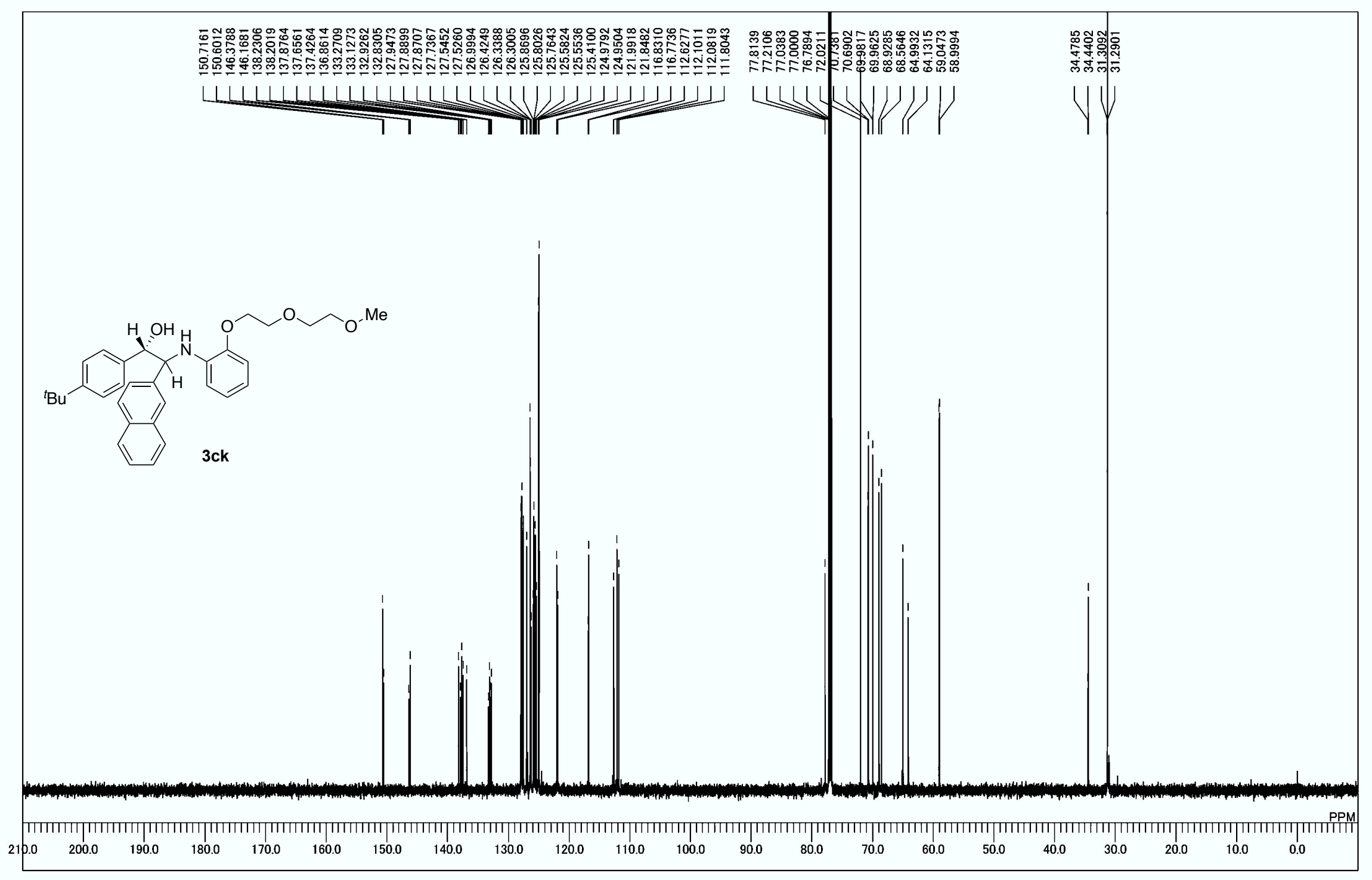

${ }^{13} \mathrm{C}$ NMR spectrum of $\mathbf{3 c k}$ 


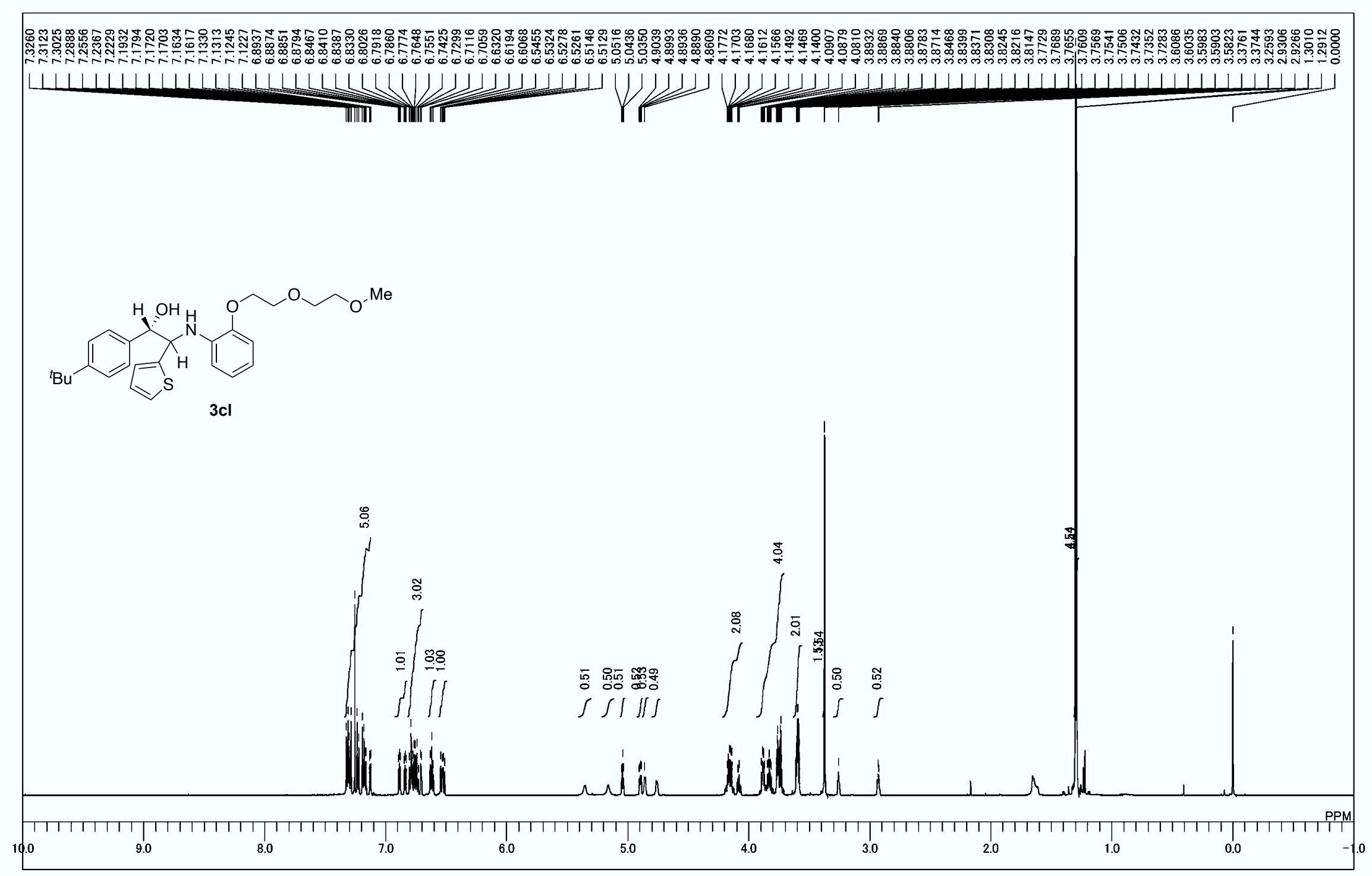

${ }^{1} \mathrm{H}$ NMR spectrum of $\mathbf{3 c l}$ 


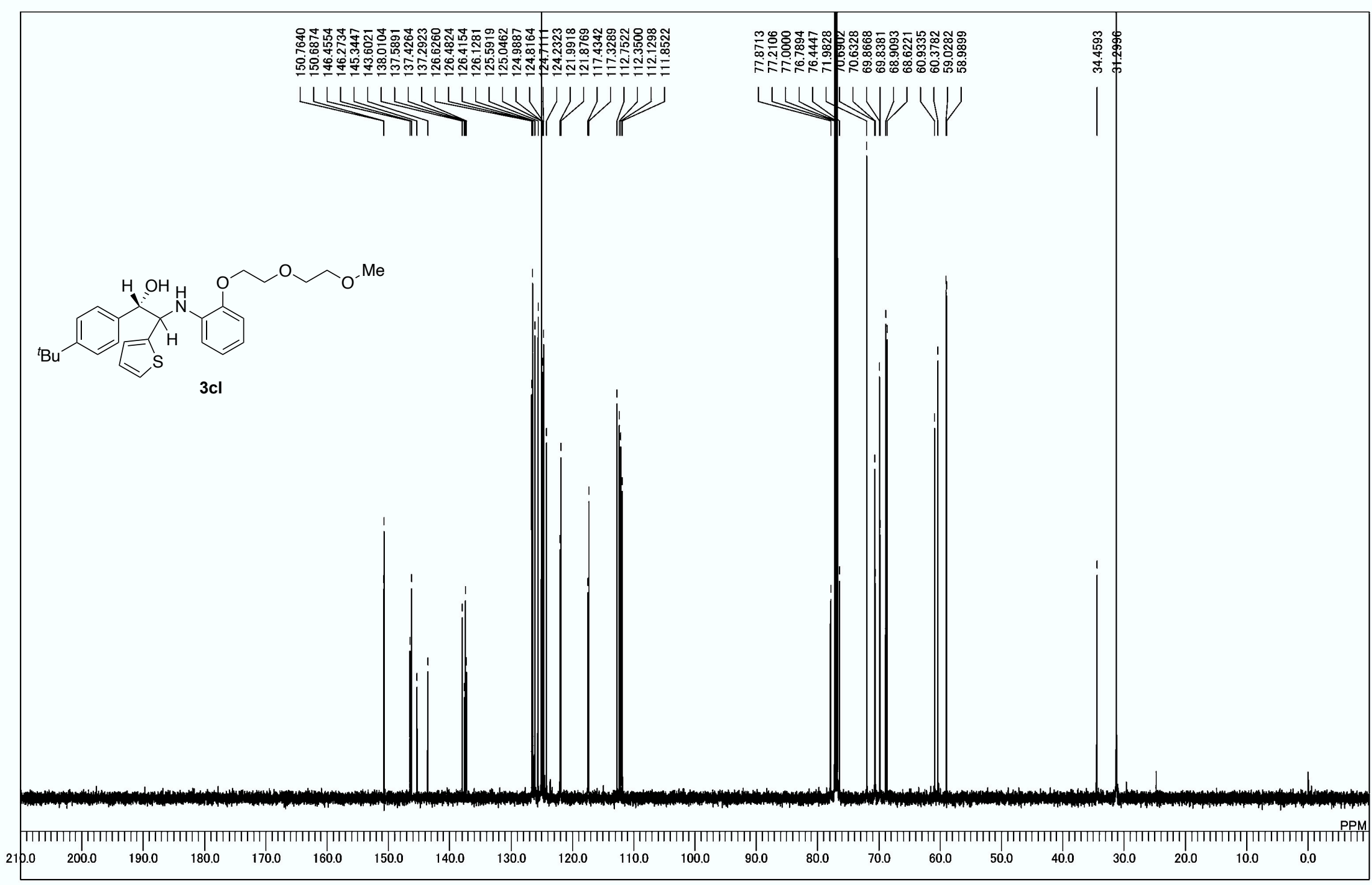

${ }^{13} \mathrm{C}$ NMR spectrum of $\mathbf{3 c l}$ 


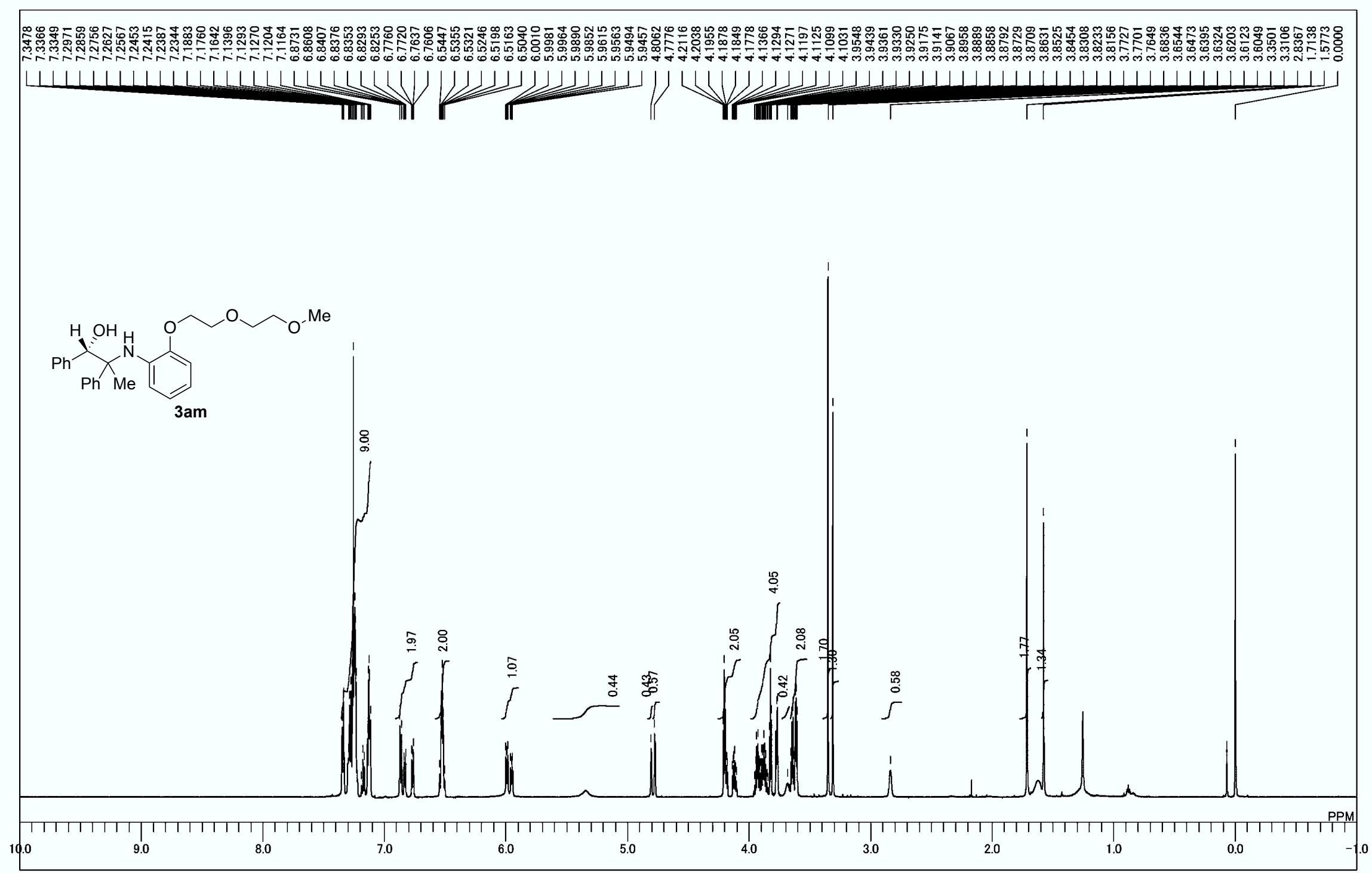

${ }^{1} \mathrm{H}$ NMR spectrum of $\mathbf{3 a m}$ 


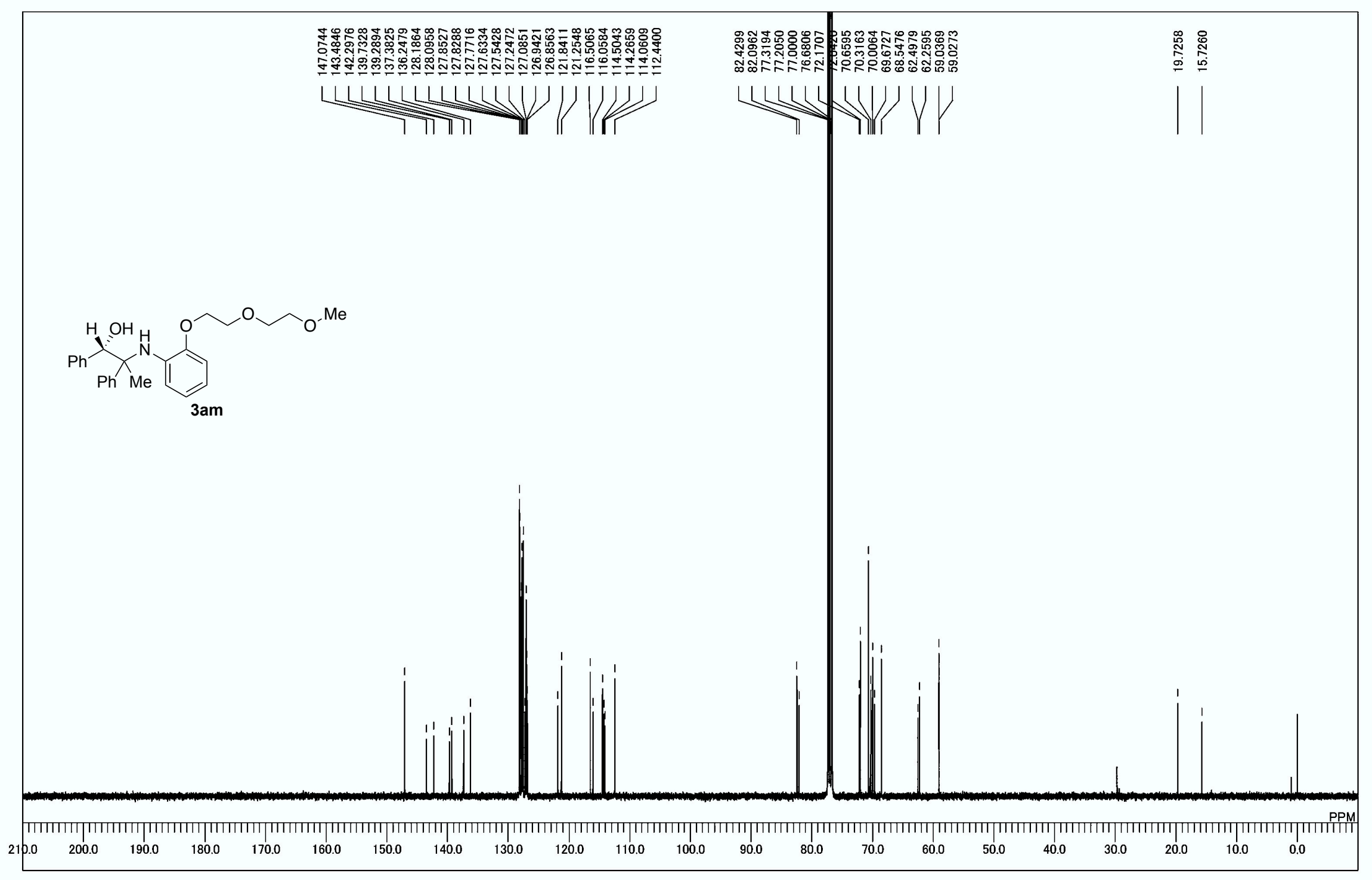

${ }^{13} \mathrm{C}$ NMR spectrum of $\mathbf{3 a m}$ 


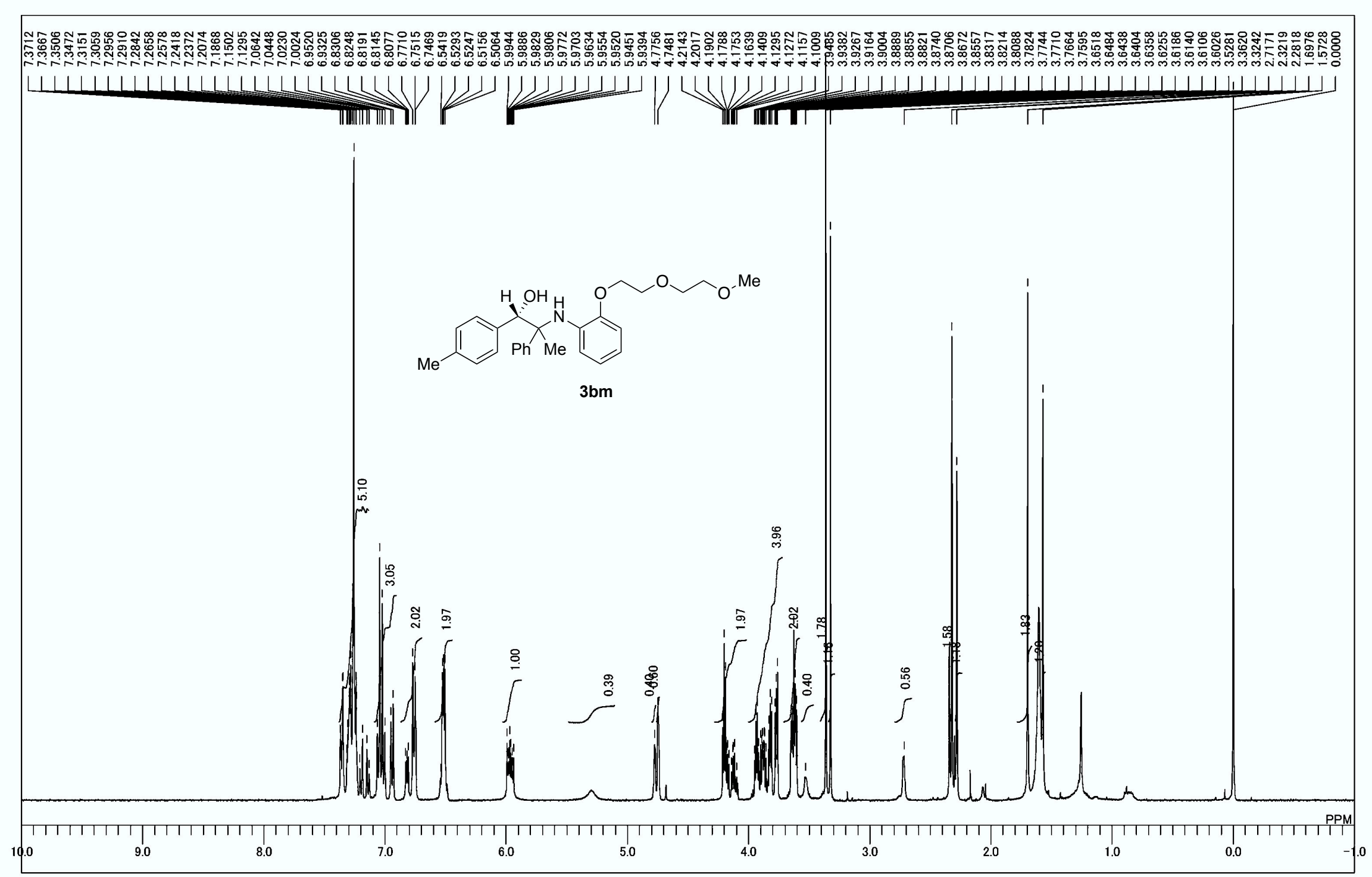

${ }^{1} \mathrm{H}$ NMR spectrum of $\mathbf{3 b m}$ 


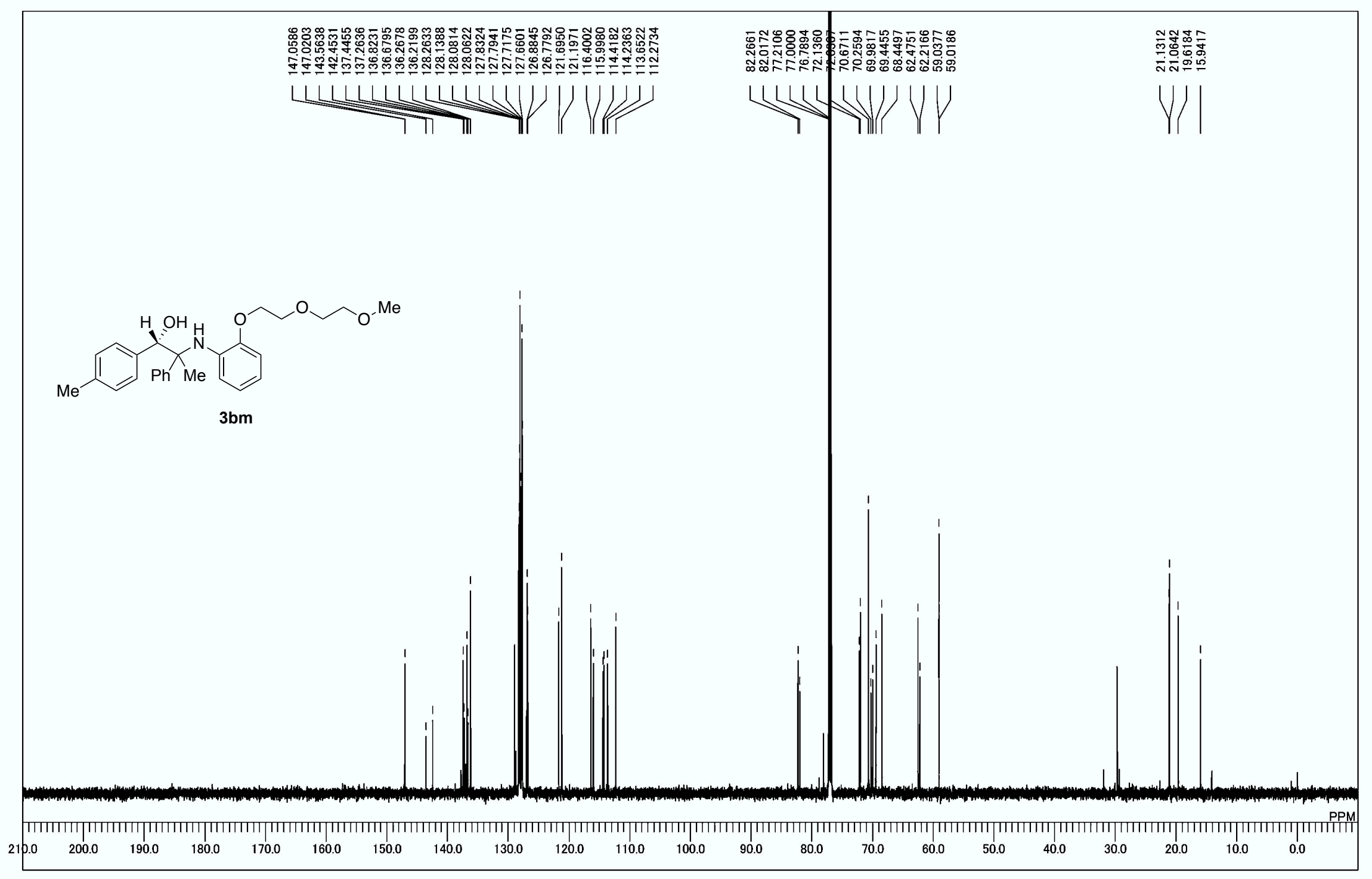

${ }^{13} \mathrm{C}$ NMR spectrum of $\mathbf{3 b m}$ 


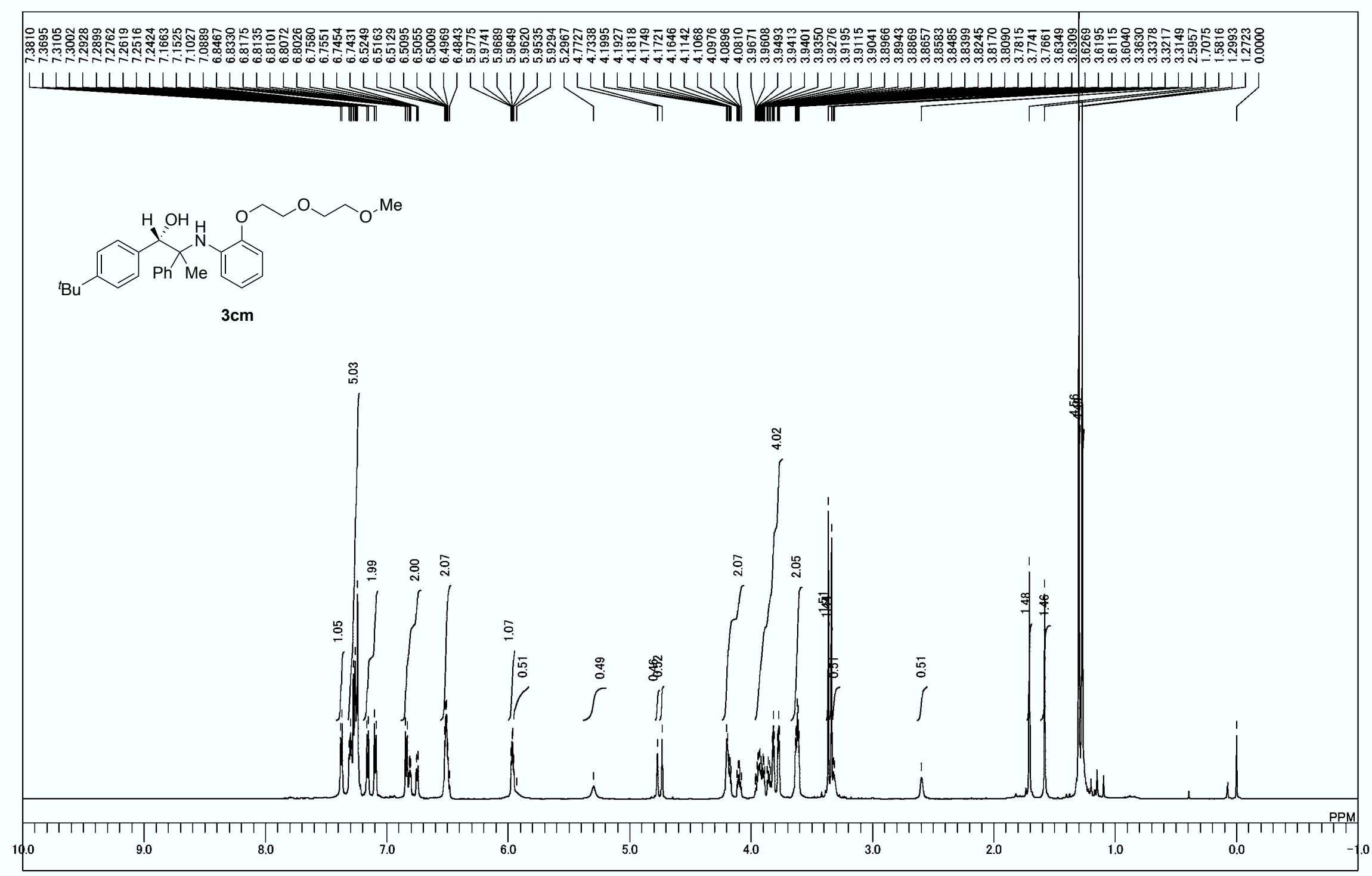

${ }^{1} \mathrm{H}$ NMR spectrum of $\mathbf{3} \mathbf{c m}$ 


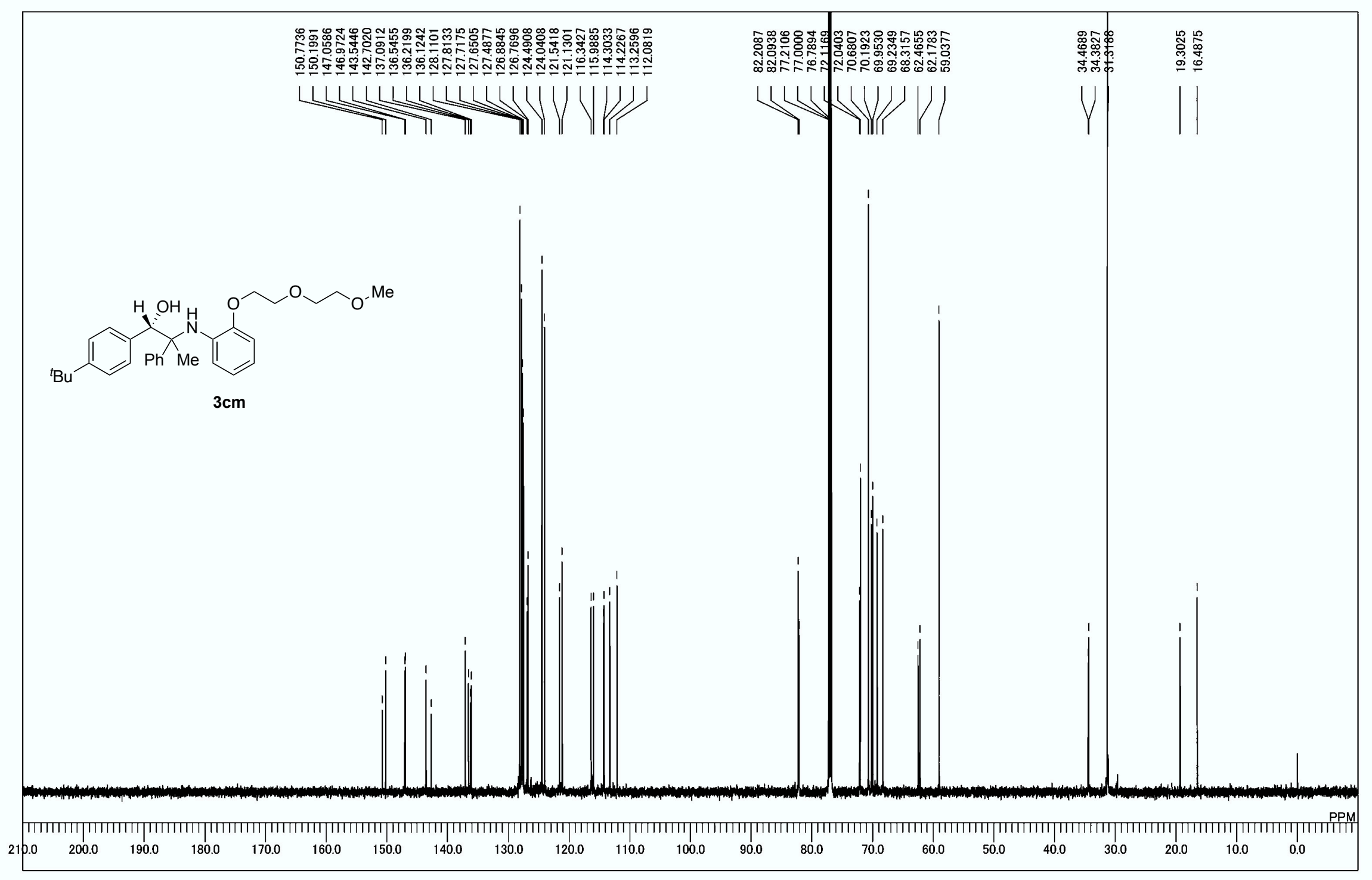

${ }^{13} \mathrm{C}$ NMR spectrum of $\mathbf{3} \mathbf{c m}$ 


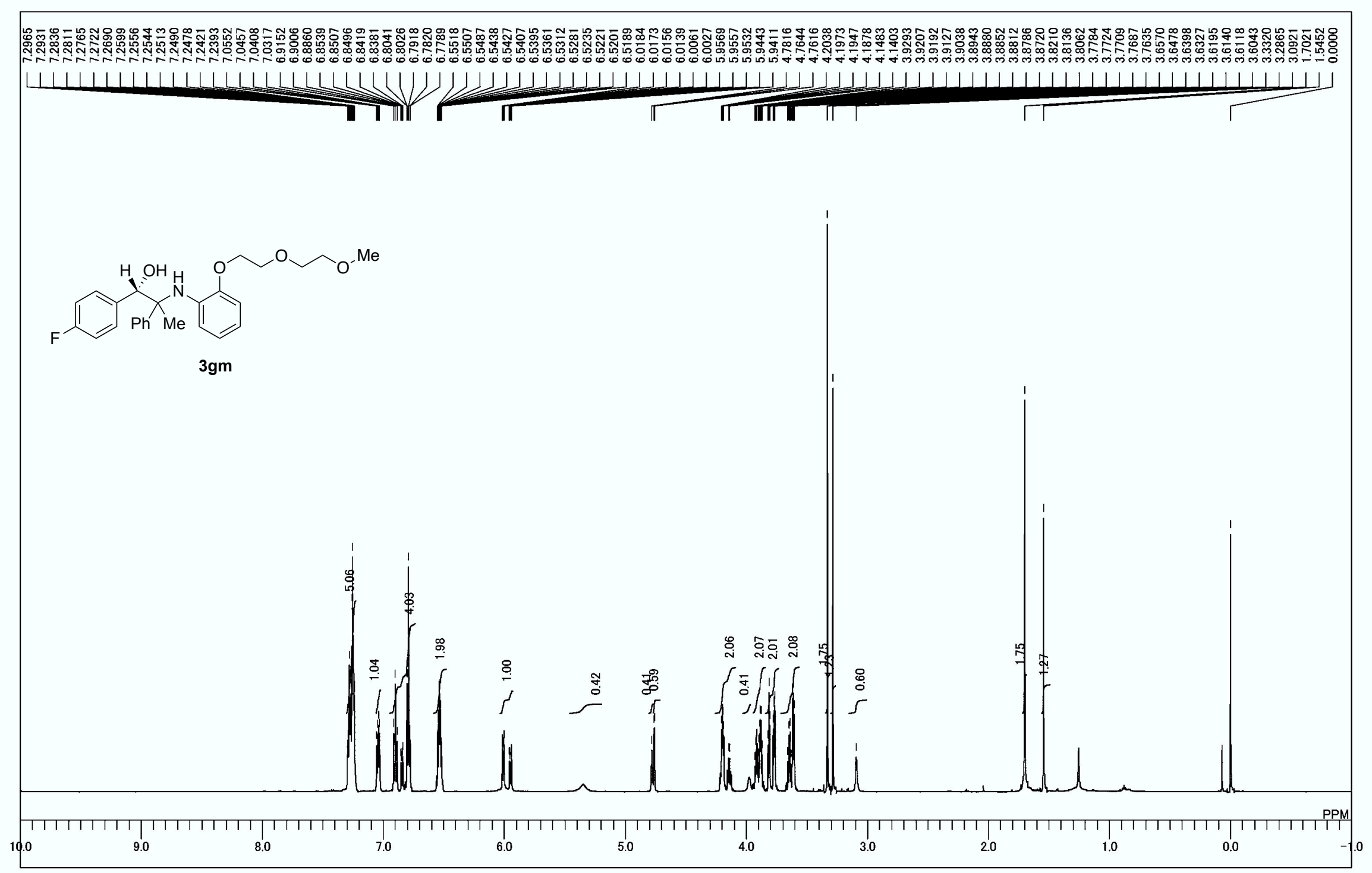

${ }^{1} \mathrm{H}$ NMR spectrum of $\mathbf{3 g m}$ 


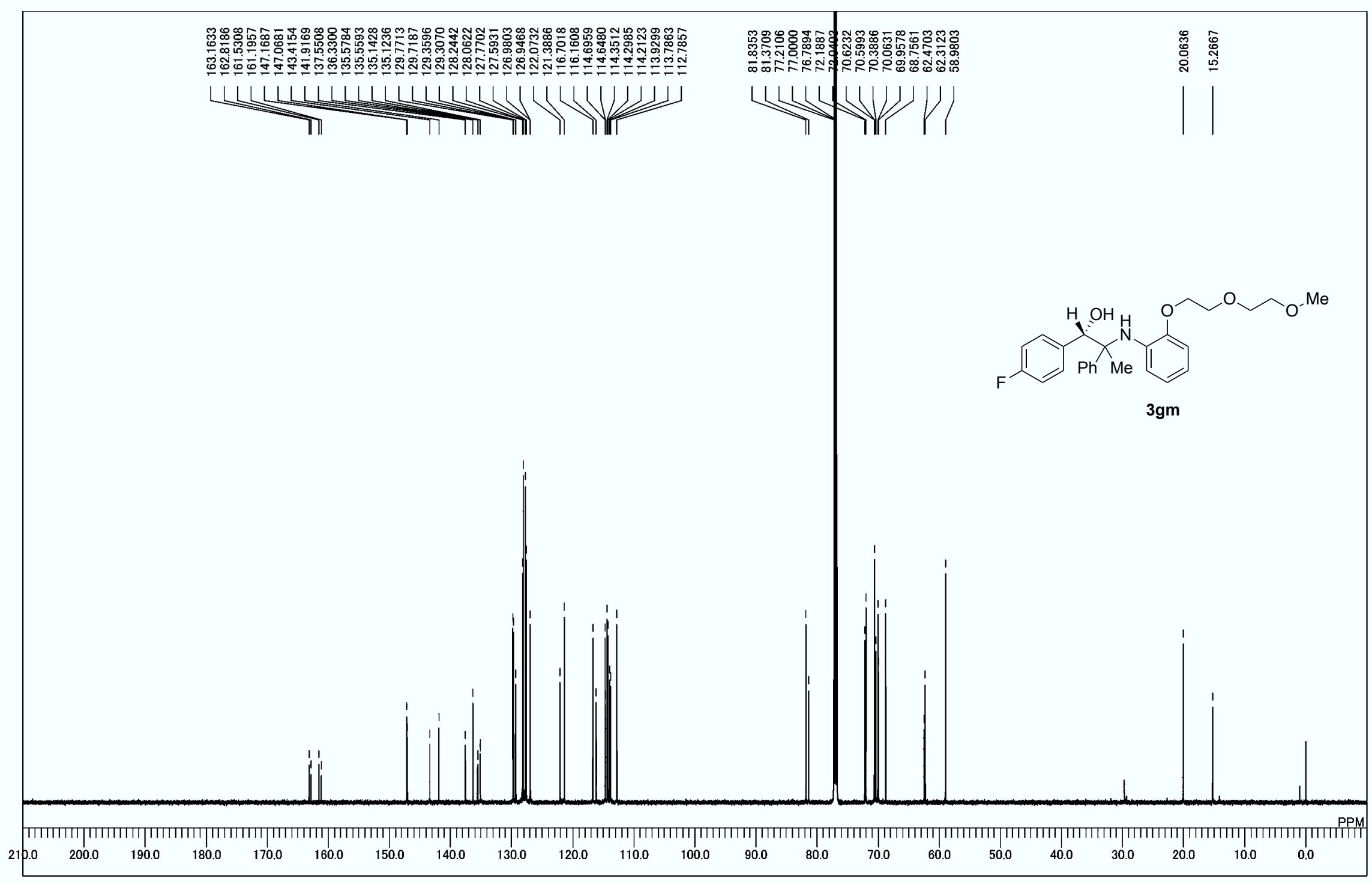

${ }^{13} \mathrm{C}$ NMR spectrum of $\mathbf{3 g m}$ 


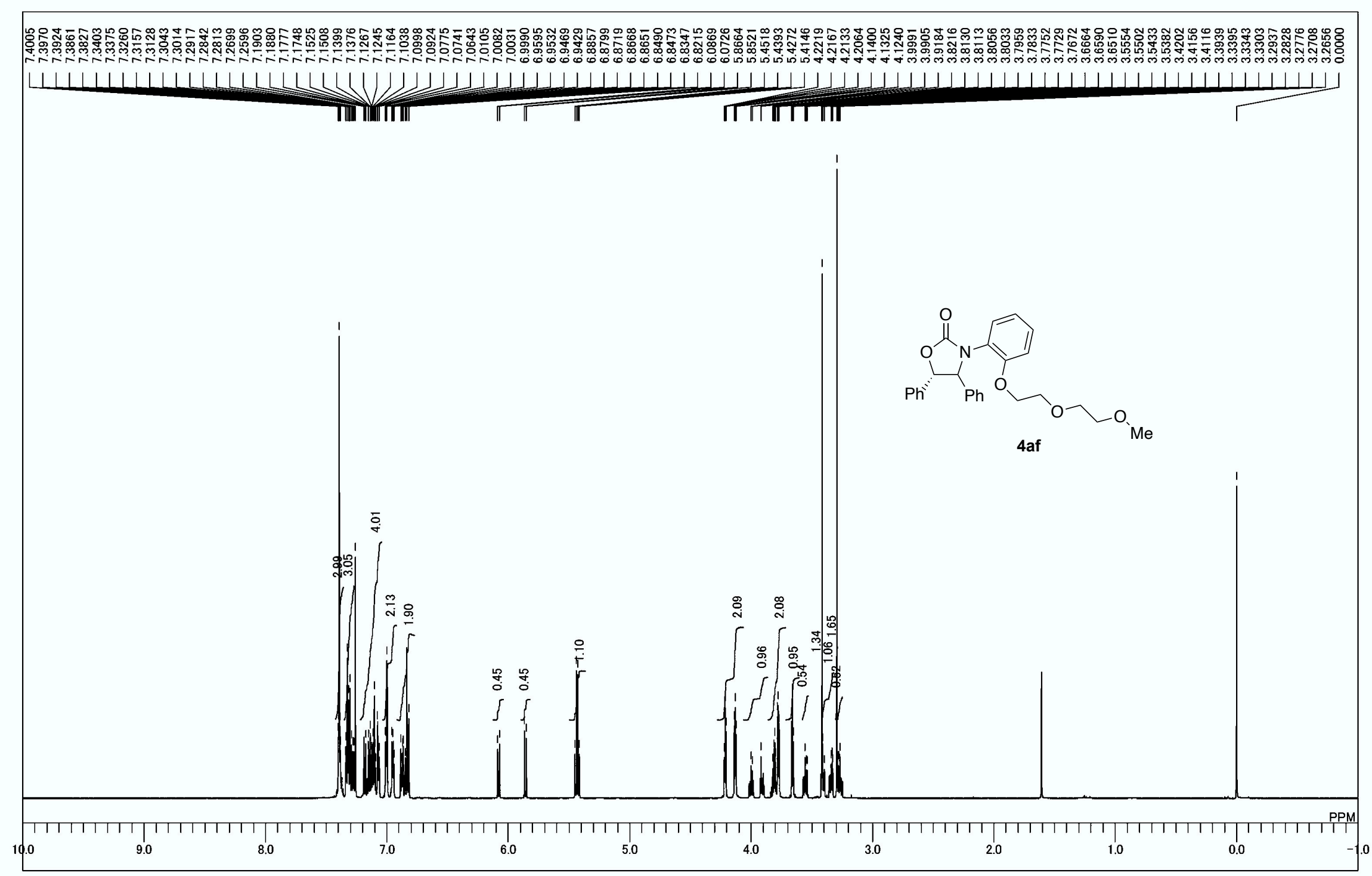

${ }^{1} \mathrm{H}$ NMR spectrum of $\mathbf{4 a f}$ 


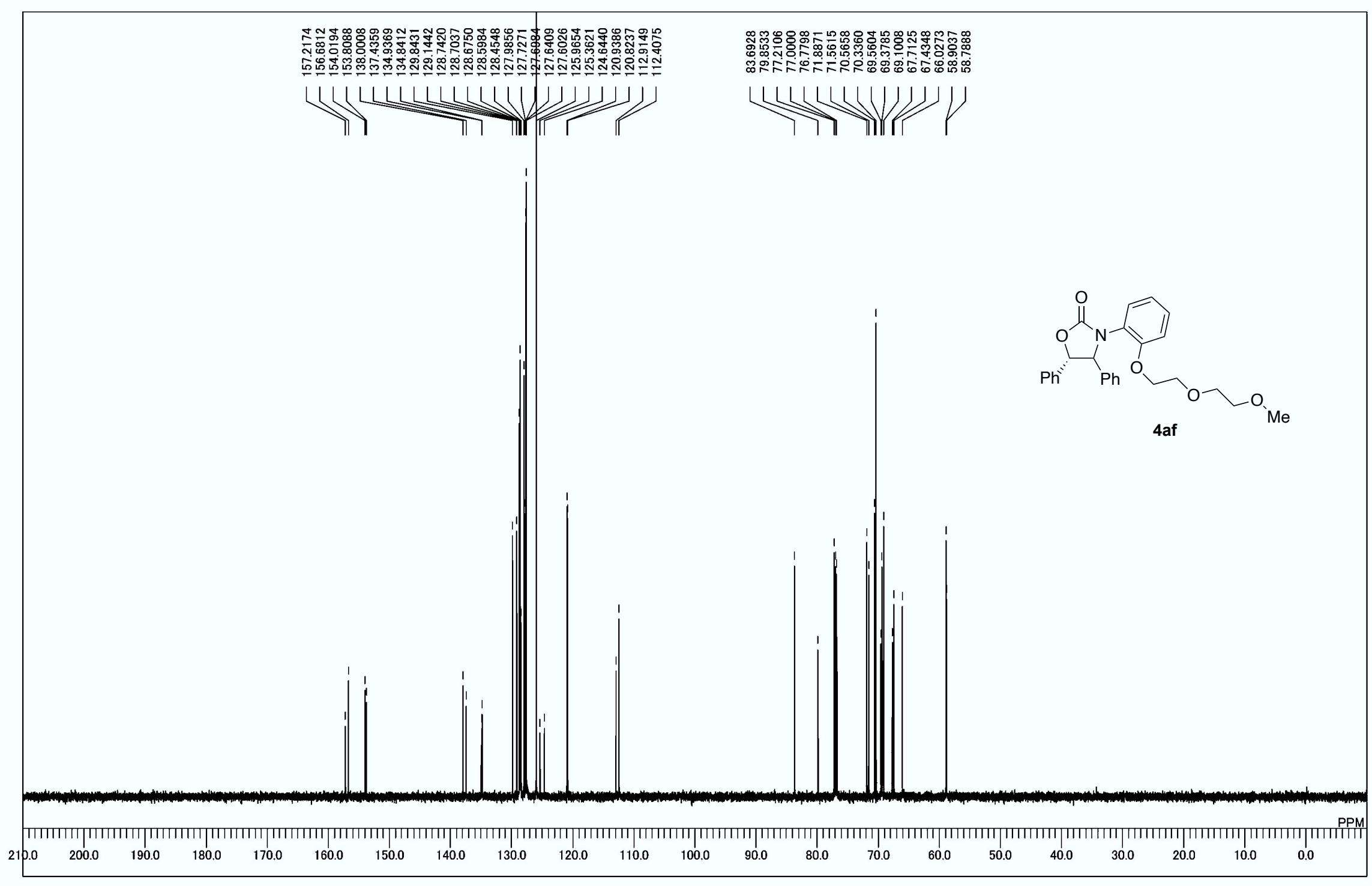

${ }^{13} \mathrm{C}$ NMR spectrum of $\mathbf{4 a f}$ 


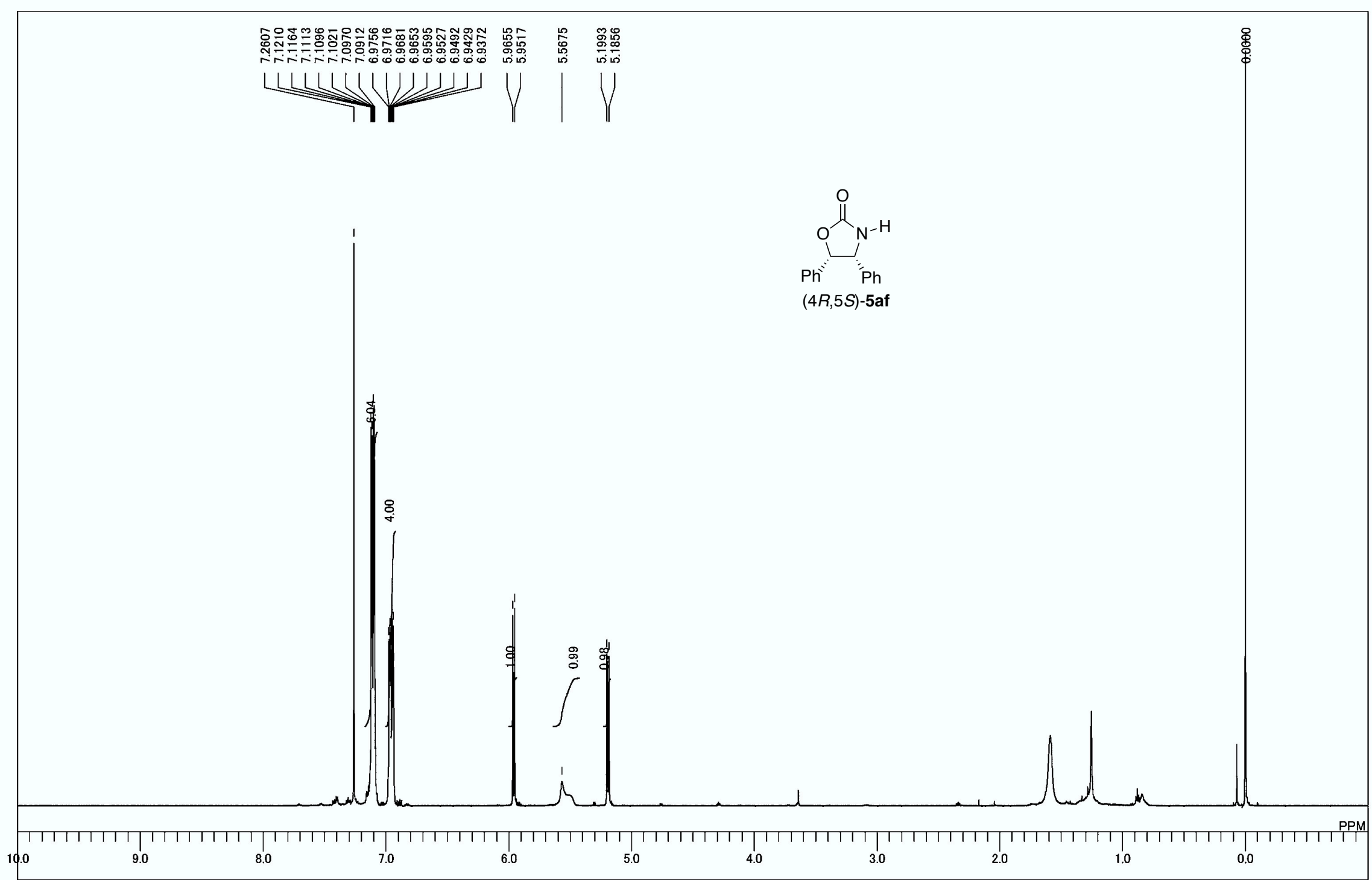

${ }^{1} \mathrm{H}$ NMR spectrum of $(4 R, 5 S)-\mathbf{5 a f}$ 


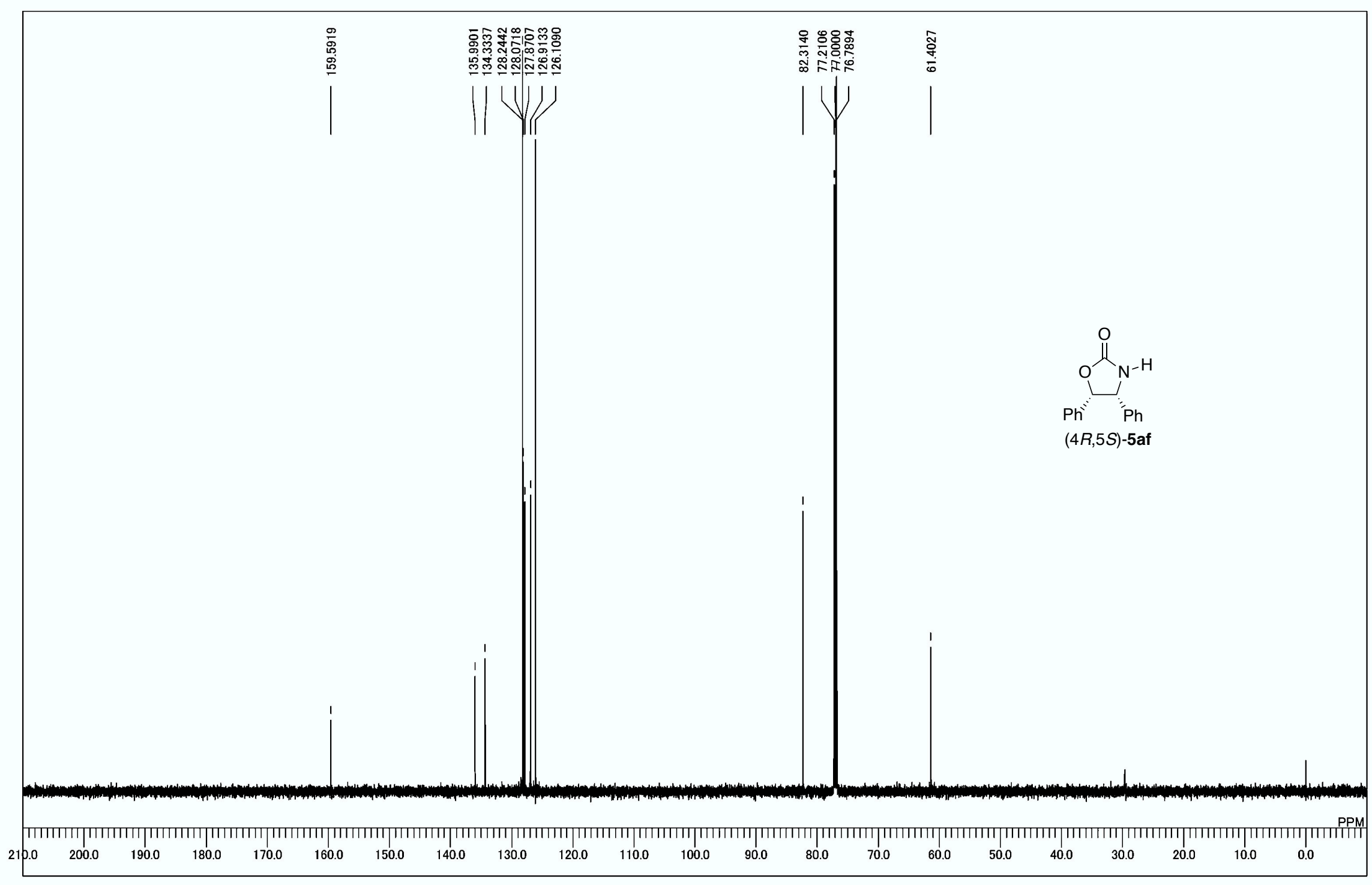

${ }^{13} \mathrm{C}$ NMR spectrum of $(4 R, 5 S)-\mathbf{5 a f}$ 


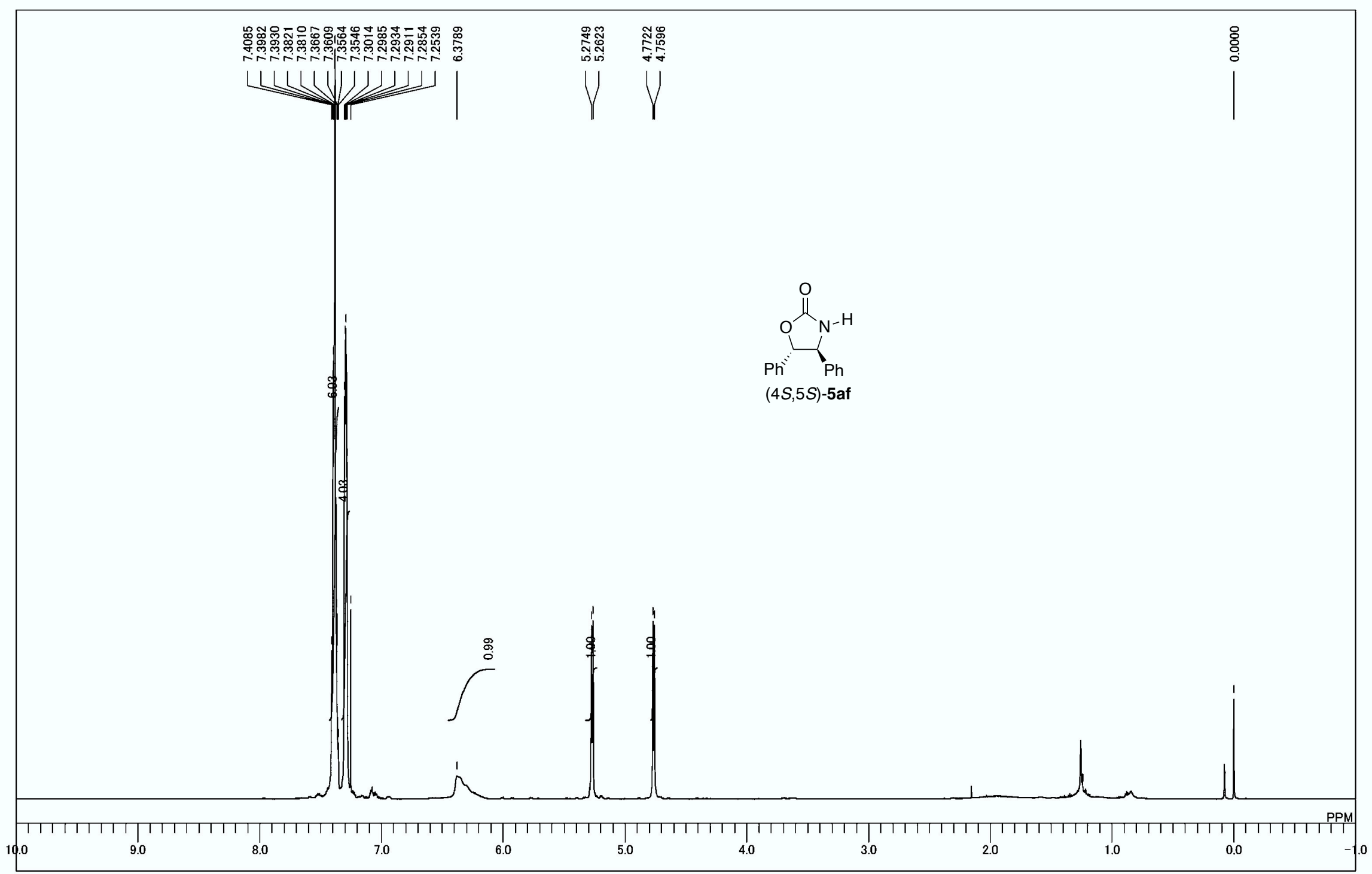

${ }^{1} \mathrm{H}$ NMR spectrum of $(4 S, 5 S)-\mathbf{5 a f}$ 


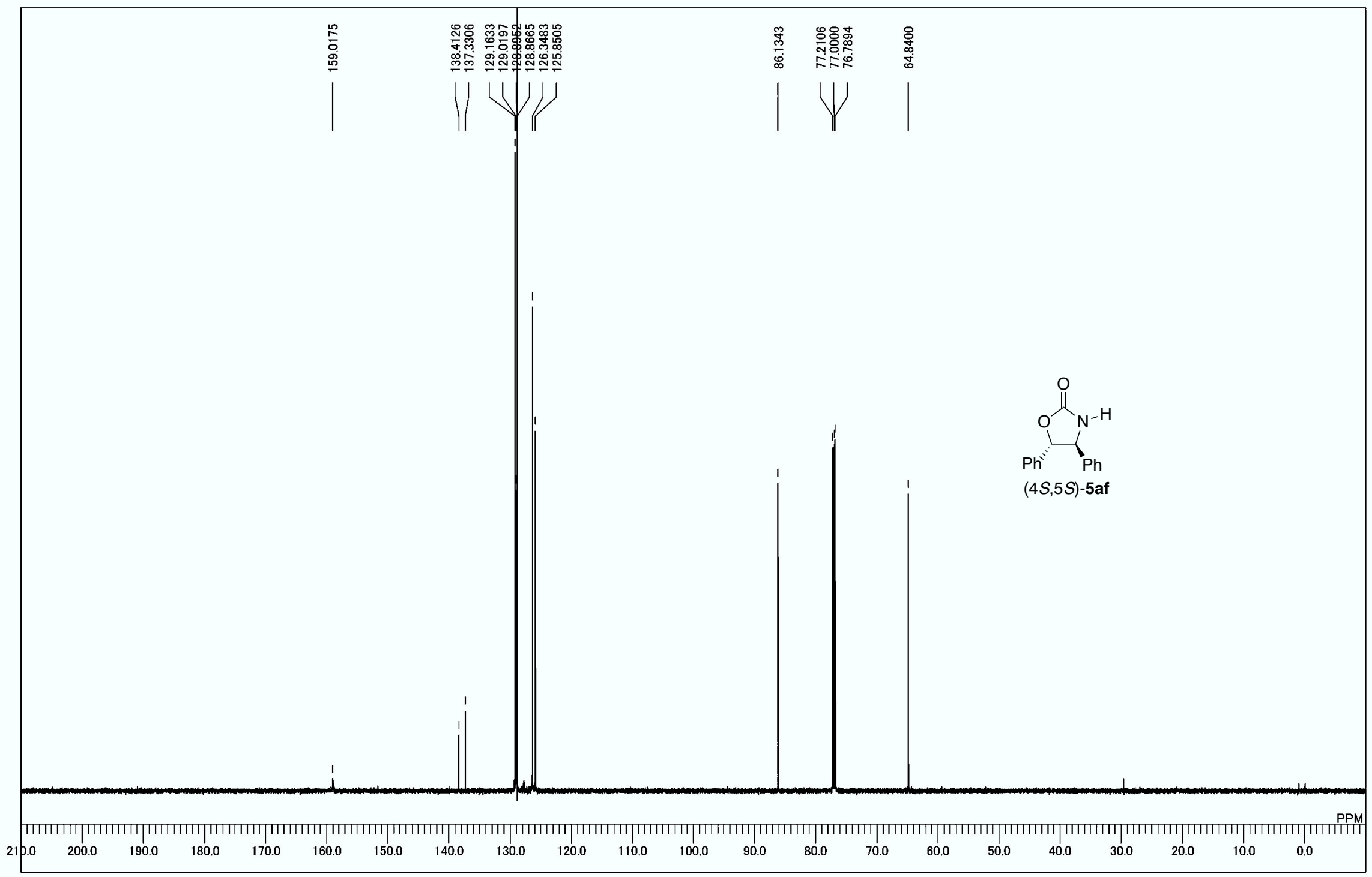

${ }^{13} \mathrm{C}$ NMR spectrum of $(4 S, 5 S)-5$ af

S111 